\title{
Supporting Information: Tunable and Practical Homogeneous Organic Reductants for Cross-Electrophile Coupling
}

David J. Charboneau, a, ${ }^{\text {a }}$ Haotian Huang,, a, ${ }^{\uparrow}$ Emily L. Barth, ${ }^{\mathrm{a}}$ Cameron C. Germe, ${ }^{\mathrm{a}}$ Nilay Hazari, ${ }^{\mathrm{a}, *}$ Brandon Q. Mercado, ${ }^{a}$ Mycah R. Uehling ${ }^{\mathrm{b}, *} \&$ Susan L. Zultanski ${ }^{\mathrm{c}, *}$

aDepartment of Chemistry, Yale University, P. O. Box 208107, New Haven, Connecticut, 06520, USA. E-mail: nilay.hazari@yale.edu.

${ }^{b}$ Merck \& Co., Inc., Discovery Chemistry, HTE and Lead Discovery Capabilities, Kenilworth, New Jersey, 07033, USA. E-mail: mycah.uehling@merck.com.

'Merck \& Co., Inc., Department of Process Research and Development, Kenilworth, New Jersey, 07033, USA. E-mail: susan_zultanski@merck.com.

"Denotes that the authors made equal contribution.

\section{Table of Contents}

SI. General Methods

S3

SII. Synthesis of Formamidinium Hexafluorophosphate Salts

SIII. Synthesis of Tetraaminoethylenes

SIV. Synthesis of Oxidized Tetraaminoethylenes

SV. Air Stability Studies of Tetraaminoethylenes

SVI. Solubility Studies of Tetraaminoethylenes

SVII. Procedure for ${ }^{1} H$ NMR Yields of Products from Cross-Electrophile Coupling

of Aryl and Alkyl Halides

SVIII. High Throughput Experimentation for Optimization of Drug-Like Aryl Halides

SIX. Procedure for ${ }^{1} H$ NMR Yields of Cross-Electrophile Coupling of Drug-Like Aryl

Halides and Alkyl Halides

SX. Reductant Screen for Intermolecular Three-Component Dicarbofunctionalization of Alkenes

SXI. Synthesis of Katritzky Salts

SXII. Characterization of Katritzky Salts

SXIII. Optimization of Cross-Electrophile Coupling of Aryl Halides and Katritzky Salts

SXIV. Reductant Screen for Cross-Electrophile Coupling of 2-Iodoanisole and Katritzky Salts 1, 3, and 4

SXV. ${ }^{1} H$ NMR Yields of Products from Cross-Electrophile Coupling of Aryl Bromides and Katritzky Salt 4

SXVI. ${ }^{1} H$ NMR Yields of Products from Cross-Electrophile Coupling of Additional Aryl Iodide Substrates and Katritzky Salt 4

SXVII. Isolation Procedure and Characterization for Products of Cross-Electrophile Coupling of Aryl Halides and Katritzky Salts

SXVIII. Procedure for $1 \mathrm{mmol}$ Scale Reaction to Form Product $\mathbf{9 f}$

SXIX. NMR Spectra of Isolated Compounds
S6

S8

$\mathrm{S} 12$

$\mathrm{S} 14$

S15

S16

$\mathrm{S} 17$

S25

S27

S29

S30

S33

S34

S37

S38

S39

S43

S44 
SXX. Cyclic Voltammetry

SXXII. Computational Details and Coordinates

S99

SXXIII. References

S123 


\section{SI. General Methods}

Experiments were performed under an atmosphere of $\mathrm{N}_{2}$ in an M-Braun glovebox or using standard Schlenk techniques, unless specified otherwise. Purging of the glovebox atmosphere was not performed between uses of pentane, benzene, toluene, diethyl ether, 1,4-dioxane and tetrahydrofuran (THF); as such, trace amounts of the solvents may have been present in the box atmosphere and intermixed in the solvent bottles.

1,4-Dioxane, $N, N$-dimethylformamide (DMF), acetonitrile, pentane, THF, benzene, dichloromethane, diethyl ether, and toluene were dried via passage through a column of activated alumina on an Inert Technologies PureSolv MD7 solvent purification system and subsequently stored under $\mathrm{N}_{2}$ unless otherwise noted. EtOH was purchased as 200 proof and used as received. In cases where dry and degassed $\mathrm{EtOH}$ was required, $\mathrm{EtOH}$ was quickly transferred from a newly opened bottle of 200 proof EtOH to a Straus flask and sparged with $\mathrm{N}_{2}$, then stored under $\mathrm{N}_{2}$. DMAc ( $N, N$-dimethylacetamide) was purchased as anhydrous $99.8 \%$ from Millipore Sigma and used without further purification. Deuterated solvents were obtained from Cambridge Isotope Laboratories and were in some cases dried prior to use. $\mathrm{C}_{6} \mathrm{D}_{6}$ and $\mathrm{CD}_{3} \mathrm{CN}$ were degassed via three consecutive freeze-pump-thaw cycles and dried by passage through a short column of neutral activated alumina and stored under an $\mathrm{N}_{2}$ atmosphere. Neutral alumina was activated by heating at $250{ }^{\circ} \mathrm{C}$ in vacuo overnight. $3 \AA$ molecular sieves were activated by heating at $250{ }^{\circ} \mathrm{C}$ in vacuo overnight. $\mathrm{CDCl}_{3}$ was degassed via three consecutive freeze-pump-thaw cycles and then dried by stirring over excess calcium hydride followed by distillation and then storage under an $\mathrm{N}_{2}$ atmosphere. DMSO- $\mathrm{d}_{6}$ was purchased in $1 \mathrm{~mL}$ ampules and used as received.

NMR spectra were recorded on Agilent-400, -500 , or $-600 \mathrm{MHz}$ spectrometers at ambient probe temperatures unless otherwise stated. Chemical shifts for ${ }^{1} \mathrm{H}$ NMR and ${ }^{13} \mathrm{C}\left\{{ }^{1} \mathrm{H}\right\}$ NMR spectra are reported with respect to residual protio solvent in $\mathrm{ppm}$. Chemical shifts for other nuclei are referenced through the gyromagnetic ratio method described by Harris et al. ${ }^{1}$

Electrochemical measurements were performed with $\mathrm{CH}$ Instruments potentiostats (models $600 \mathrm{D} / 650 \mathrm{D}$ ) using a $3 \mathrm{~mm}$ glassy carbon working electrode, a platinum wire auxiliary electrode, and a silver wire pseudo-reference electrode. To prepare the reference electrode, $0.1 \mathrm{M}$ tetrabutylammonium hexafluorophosphate in DMF was added to a jacketed compartment (separated from solution with a glass frit) and allowed to equilibrate for 24 hours prior to use. A silver wire roughened with 600 grit sandpaper was then immersed in the jacketed compartment. 
Glassy carbon working electrodes were polished for 30-60 seconds using 0.05 $\mu \mathrm{m}$ alumina and then rinsed with DI water and dried before every measurement. For cyclic voltammetry measurements, the potential was compensated for the internal resistance of the cell, and ferrocene was added as an internal reference.

Mass spectrometric measurements were performed with a Thermo Fisher QExactive Orbitrap LC-MS system, equipped with a Dionex UltraMate 3000 UHPLC, consisting of an LPG3400SD pump unit, a WPS-3000 autosampler, and a Column Oven TCC-3000. Mass spectra (MS and MS/MS) were recorded with the QExactive Orbitrap mass spectrometer. Electrospray was used for desolvatization and ionization, with the electrospray needle held at $+3.5 \mathrm{kV}$. Compressed air was used as desolvatization gas, with capillary temperature at $250{ }^{\circ} \mathrm{C}$, probe heater temperature at $400{ }^{\circ} \mathrm{C}$, sheath gas at $47.5 \mathrm{~L} / \mathrm{min}$, and aux gas flow at $11 \mathrm{~L} / \mathrm{min}$. Resolution was set to 35,000 $\mathrm{M} / \Delta \mathrm{M}$. Mass spectra were recorded in the range of 150 to $2000 \mathrm{~m} / \mathrm{z}$ in positive ion mode. Measurements and data post-processing were performed with Thermo Xcalibur 4.1.31.9. Two methods were used for sample preparation and injection. The first method was used for all tetraaminoethylene compounds. The second method was used for all other compounds. Method 1: Samples were prepared in a glove box in dry and degassed THF and loaded in a gas tight, sample lock syringe, Hamilton 1725 SL SYR, for transport. Capillaries on the mass spectrometer were flushed with dry and degassed THF prior to sample injection. Samples were held at room temperature and continuously injected using a syringe pump at $15 \mu \mathrm{L} / \mathrm{min}$. Method 2: The samples were prepared on the benchtop in $\mathrm{CH}_{3} \mathrm{CN}$ and held at room temperature in the autosampler compartment. $1 \mathrm{uL}$ of each solution was directly injected into the flow path of the LC system without column separation. An isocratic flow was used with a constant rate of $0.3000 \mathrm{~mL} / \mathrm{min}$ : 20\% Solvent A: Water, HPLC grade Chromasolv, with 0.1\% Formic Acid; 80\% Solvent B: Acetonitrile, HPLC grade Chromasolv, with 0.1\% Formic Acid.

Chemicals that were purchased from commercial suppliers were used as received unless otherwise stated. 4,4'-di-tert-butyl-2,2'-bipyridine (dtbbpy) was purchased from Sigma Aldrich or Santa Cruz at $>97 \%$ purity. Aryl and alkyl halide substrates were purchased at $\geq 97 \%$ purity. All liquid aryl and alkyl substrates were degassed by sparging with nitrogen or by three consecutive freeze-pump-thaw cycles and then handled inside of a nitrogen filled glovebox. Liquid aryl and alkyl halide substrates that had a yellow color instead of being colorless were purified by passage through a short column of neutral activated alumina prior to use. Tetrakis(dimethylamino)ethylene 
(TDAE) was purchased from Sigma-Aldrich, TCI, AstaTech, or Santa Cruz. Diisopropylamine was purchased as $>99.95 \%$ from Millipore Sigma. ${ }^{n} \mathrm{BuLi}$ was purchased as $2.5 \mathrm{M}$ in hexanes from Millipore Sigma. Sodium hexafluorophosphate was purchased from Oakwood Chemicals. Piperidine was purchased as $>99.5 \%$ from Millipore Sigma. Morpholine was purchased from Alfa Aesar as $>99 \%$. Pyrrolidine was purchased from Oakwood Chemicals as $>99 \%$. Dimethylcarbamoyl chloride as purchased from Millipore Sigma as $>98 \%$. 4aminotetrahydropyran was purchased from Oakwood chemicals as $>98 \%$. 4-tert-butylbenzylamine was purchased from TCI as $>98 \%$. 4-methoxybenzylamine was purchased from TCI as $>97 \%$. 4(trifluoromethyl)benzylamine was purchased from TCI as $>98 \%$. 3-phenylpropylamine was purchased from Alfa Aesar as $>98 \%$. 2,4,6-triphenylpyrylium tetrafluoroborate was purchased from Oakwood chemicals as $>95 \%$. $3 \AA$ molecular sieves were purchased from Alfa Aesar.

The following compounds were synthesized according to literature procedures: (dtbbpy) $\mathrm{Ni}^{\mathrm{II}} \mathrm{Br}_{2},{ }^{2} \quad \mathrm{~N}, \mathrm{~N}, \mathrm{~N}^{\prime}, \mathrm{N}^{\prime}$-tetramethylformamidinium chloride, ${ }^{3}$ and 2,4,6-tris(4methoxyphenyl)pyrylium tetrafluoroborate. ${ }^{4}$ 


\section{SII. Synthesis of Formamidinium Hexafluorophosphate Salts}

General Procedure A:

The procedure was adapted from a literature report. ${ }^{5}$ In a glovebox under an $\mathrm{N}_{2}$ atmosphere, to a Schlenk flask charged with a magnetic stir bar was added N,N,N',N'tetramethylformamidinium chloride (1 equiv.), and degassed EtOH (7.5 M). The Schlenk flask was capped, attached to a Schlenk line using standard techniques, and fitted with a rubber septum. To the flask was added the corresponding amine ( 3 equiv.), which was degassed by sparging with $\mathrm{N}_{2}$ prior to the addition, via syringe transfer. The rubber septum was removed from the Schlenk flask, which was then fitted with a reflux condenser against a positive pressure of $\mathrm{N}_{2}$. The reflux condenser was fitted with a rubber septum and a gas outlet attached to a bubbler containing a $1 \mathrm{M}$ $\mathrm{HCl}$ solution (to scrub out dimethylamine liberated during the reaction) was inserted into the top of the reflux condenser. The reaction was heated at reflux for 5 hours then allowed to cool to room temperature and volatiles were removed in vacuo. The resulting solid was opened to air and dissolved in $0{ }^{\circ} \mathrm{C}$ water, then quickly transferred a round bottom flask charged with a magnetic stir bar containing a nearly saturated solution of sodium hexafluorophosphate (1.2 equiv.) in water at room temperature, resulting in the formation of a precipitate. The solution was stirred for one minute at room temperature then filtered through a medium-fritted glass funnel, and the resulting solids were washed with copious water and dried in vacuo, resulting in the desired formamidinium hexafluorophosphate salt product.

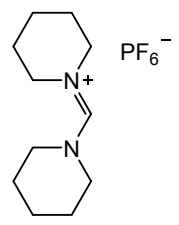

Piperidin-1-ylmethylidenepiperidinium hexafluorophosphate (2c-Pi): Synthesized according to general procedure A. $15.5 \mathrm{~g}(65 \%)$. The NMR spectra are consistent with a previous literature report. ${ }^{5}$

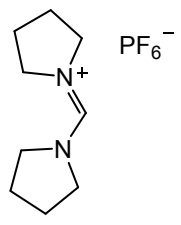

Pyrrolidin-1-ylmethylidenepyrrolidinium hexafluorophosphate (2c-Py): Synthesized according to general procedure A. $20.1 \mathrm{~g}(87 \%)$. The NMR spectra are consistent with a previous literature report. ${ }^{5}$ 


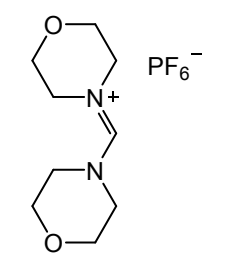

Morpholin-1-ylmethylidenemorpholinium hexafluorophosphate (2c-M): Synthesized according to general procedure A. $46.3 \mathrm{~g}(61 \%)$. The NMR spectra are consistent with a previous literature report. ${ }^{6}$

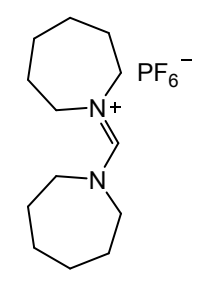

Azepan-1-ylmethylideneazepanium hexafluorophosphate (2c-Az): Synthesized according to general procedure A. 4.4 g (71\%). ${ }^{1} \mathrm{H}$ NMR $\left(400 \mathrm{MHz}, \mathrm{CD}_{3} \mathrm{CN}\right) \delta 7.50(\mathrm{~s}, 1 \mathrm{H}), 3.67$ (t, J = $6.0 \mathrm{~Hz}$, 4H), $3.56(\mathrm{t}, \mathrm{J}=6.1 \mathrm{~Hz}, 4 \mathrm{H}), 1.80(\mathrm{~m}, 8 \mathrm{H}), 1.64(\mathrm{~m}, 8 \mathrm{H}) .{ }^{13} \mathrm{C}\left\{{ }^{1} \mathrm{H}\right\} \mathrm{NMR}\left(100 \mathrm{MHz}, \mathrm{CD}_{3} \mathrm{CN}\right) \delta$ 156.13, 57.67, 49.74, 28.71, 27.71, 26.05, 25.50. ${ }^{31} \mathrm{P}\left\{{ }^{1} \mathrm{H}\right\}\left(161.835 \mathrm{MHz}, \mathrm{CD}_{3} \mathrm{CN}\right) \delta-145.5$ (sep, $\mathrm{J}=706.5 \mathrm{~Hz}) .{ }^{19} \mathrm{~F}\left(376.14 \mathrm{MHz}, \mathrm{CD}_{3} \mathrm{CN}\right) \delta 73.81(\mathrm{~d}, \mathrm{~J}=706.3 \mathrm{~Hz}) .(\mathrm{HRMS}) \mathrm{TOF} \mathrm{MS} \mathrm{ES}^{+}(\mathrm{m} / \mathrm{z})$ $[\mathrm{M}]^{+}$calculated for $\left[\mathrm{C}_{13} \mathrm{H}_{25} \mathrm{~N}_{2}\right]^{+}$209.2012; found 209.2014. 


\section{SIII. Synthesis of Tetraaminoethylenes}

Preparation of Lithium Diisopropylamide (LDA) Solution:

All LDA solutions were prepared immediately prior to use. The following procedure details the preparation of LDA for the synthesis of TPiE as a representative procedure. A $100 \mathrm{~mL}$ Schlenk flask was removed from an oven at $130{ }^{\circ} \mathrm{C}$ and allowed to cool to room temperature under vacuum, then put under a positive pressure of $\mathrm{N}_{2}$ using standard Schlenk line technique. The Schlenk flask was fitted with a rubber septum, then $30 \mathrm{~mL}$ of dry, degassed THF and $7.2 \mathrm{~mL}$ dry, degassed diisopropylamine (51.32 mmol, 1.2 equiv.) were added. The flask was cooled to $-78{ }^{\circ} \mathrm{C}$ then 15.9 $\mathrm{mL} n \operatorname{BuLi}(2.7 \mathrm{M}$ in hexanes, $42.9 \mathrm{mmol}, 1$ equiv.) was added. The flask was allowed to warm up to room temperature and the LDA solution was used in its entirety within 30 minutes of preparation.

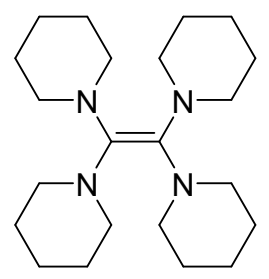

Tetrakis(piperidino)ethylene (TPiE): A $500 \mathrm{~mL}$ round bottom flask was charged with a magnetic stir bar and piperidin-1-ylmethylidenepiperidinium hexafluorophosphate (14.04 g, 42.9 mmol, 1 equiv.). The flask was dried in vacuo for 1 hour and then placed under an atmosphere of $\mathrm{N}_{2}$. Dry, degassed THF $(30 \mathrm{~mL})$ was added, and the flask was cooled to $-78^{\circ} \mathrm{C}$. LDA (42.9 mmol, 1 equiv.; see preparation of LDA above) was cooled to $-78^{\circ} \mathrm{C}$ and then added to the reaction flask via cannula transfer. The reaction mixture was allowed to warm to room temperature and left to stir under a positive pressure of $\mathrm{N}_{2}$ for 4 hours, during which time the reaction mixture turned homogeneous and pale brown. The reaction flask was opened to air and quickly transported to a rotary evaporator, where the volatiles were removed in vacuo, resulting in a pale yellow solid. Hexanes $(100 \mathrm{~mL})$ was added to the resulting solid, and the mixture was filtered through a $60 \mathrm{~mL}$ medium-fritted glass funnel. The remaining contents of the round bottom flask and solids on the fritted funnel were washed with an additional $50 \mathrm{~mL}$ hexanes. The filtrate was transferred to a 500 $\mathrm{mL}$ round bottom flask and the volatiles removed in vacuo on a rotary evaporator, resulting in a yellow solid. A magnetic stir bar was added to the round bottom flask, which was then fitted with a rubber septum. The flask was left under active vacuum for one hour and then backfilled with $\mathrm{N}_{2}$. Three 5-10 second cycles of vacuum followed by backfilling with $\mathrm{N}_{2}$ were then performed. 
Subsequently, degassed EtOH $(50 \mathrm{~mL})$ was added to the flask via cannula transfer, and the contents stirred under a positive pressure of $\mathrm{N}_{2}$ until a fine white powder was suspended in a yellow solution (about 20 minutes). Degassed $\mathrm{H}_{2} \mathrm{O}(150 \mathrm{~mL})$ was added to the flask via cannula transfer, the rubber septum was removed, and the solution was filtered through a $60 \mathrm{~mL}$ medium-fritted glass funnel. The solid was washed with $3: 1 \mathrm{H}_{2} \mathrm{O}: \mathrm{EtOH}(3 \times 30 \mathrm{~mL})$ and then quickly transferred to a glass scintillation vial and dried in vacuo. Once dry, the product was stored in a glovebox under an $\mathrm{N}_{2}$ atmosphere as a pale yellow solid, $4.6 \mathrm{~g}(60 \%) .{ }^{7}{ }^{1} \mathrm{H}$ NMR $\left(400 \mathrm{MHz}, \mathrm{C}_{6} \mathrm{D}_{6}\right) \delta 2.98$ (br s, 16H, N$\mathrm{CH}_{2}-\mathrm{C}$ ), 1.53 (br s, 24H, C-CH $\left.2-\mathrm{C}\right) .{ }^{13} \mathrm{C}\left\{{ }^{1} \mathrm{H}\right\}$ NMR $\left(150 \mathrm{MHz}, \mathrm{C}_{6} \mathrm{D}_{6}\right) \delta$ 134.6, 51.6, 27.4, 25.8. (HRMS) TOF MS ES ${ }^{+}(\mathrm{m} / \mathrm{z})[\mathrm{M}]^{+}$calculated for $\left[\mathrm{C}_{22} \mathrm{H}_{40} \mathrm{~N}_{4}\right]^{+}$360.3247; found 360.3229. Anal. Calcd. for $\mathrm{C}_{22} \mathrm{H}_{40} \mathrm{~N}_{4}$ : C, 73.28; H, 11.18; N, 15.54. Found: C, 73.41; H, 11.42; N, 15.55.

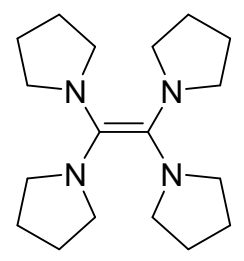

Tetrakis(pyrrolidino)ethylene (TPyE): A $500 \mathrm{~mL}$ Schlenk flask was charged with a magnetic stir bar and pyrrolidin-1-ylmethylidenepyrrolidinium hexafluorophosphate (19.5 g, $65.4 \mathrm{mmol}, 1$ equiv.). The flask was dried in vacuo for 1 hour and then placed under an atmosphere of $\mathrm{N}_{2}$. Dry, degassed THF ( $48 \mathrm{~mL})$ was added, and the flask was cooled to $-78^{\circ} \mathrm{C}$. LDA $(65.4 \mathrm{mmol}, 1$ equiv.; see preparation of LDA above) was cooled to $-78^{\circ} \mathrm{C}$ and then added to the reaction flask via cannula transfer. The reaction mixture was allowed to warm to room temperature while stirring and left to stir at room temperature under a positive pressure of $\mathrm{N}_{2}$ for 4 hours, during which time the reaction mixture turned brown and remained heterogeneous. Without exposing the reaction mixture to air, the volatiles were removed in vacuo, then dry, degassed pentane $(200 \mathrm{~mL})$ was added to the flask under $\mathrm{N}_{2}$. The resulting mixture was filtered under $\mathrm{N}_{2}$, and the volatiles were removed from the filtrate, resulting in a sticky yellow solid. The crude product mixture was transferred to a glovebox containing an $\mathrm{N}_{2}$ atmosphere and recrystallized from dry degassed $\mathrm{Et}_{2} \mathrm{O}$ at $-35{ }^{\circ} \mathrm{C}$ to give a white solid, $2.105 \mathrm{~g}(21 \%) .{ }^{1} \mathrm{H}$ NMR (400 MHz, $\left.\mathrm{C}_{6} \mathrm{D}_{6}\right) \delta 3.07$ (t, J = 6.4 Hz, $16 \mathrm{H}), 1.77(\mathrm{t}, \mathrm{J}=6.4 \mathrm{~Hz}, 16 \mathrm{H}) .{ }^{13} \mathrm{C}\left\{{ }^{1} \mathrm{H}\right\} \mathrm{NMR}\left(100 \mathrm{MHz}, \mathrm{C}_{6} \mathrm{D}_{6}\right) \delta 123.5,49.5,26.2$. (HRMS) TOF MS ES $(\mathrm{m} / \mathrm{z})[\mathrm{M}]^{+}$calculated for $\left[\mathrm{C}_{18} \mathrm{H}_{32} \mathrm{~N}_{4}\right]^{+}$304.2621; found 304.2612. Anal. Calcd. for $\mathrm{C}_{18} \mathrm{H}_{32} \mathrm{~N}_{4}:$ C, 71.01; H, 10.59; N, 18.40. Found: C, 70.67; H, 10.79; N, 18.37. 


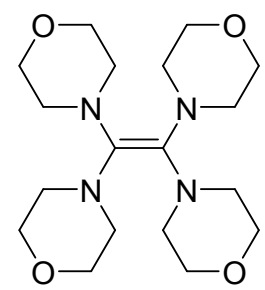

Tetrakis(morpholino)ethylene (TME): A $500 \mathrm{~mL}$ Schlenk flask was charged with a magnetic stir bar and morpholin-1-ylmethylidenemorpholinium hexafluorophosphate (37.05 g, 0.112 mol, 1 equiv.). The flask was dried in vacuo for 1 hour and then placed under an atmosphere of $\mathrm{N}_{2}$. Dry, degassed THF $(88 \mathrm{~mL})$ was added, and the flask was cooled to $-78^{\circ} \mathrm{C}$. LDA $(0.112 \mathrm{~mol}, 1$ equiv.; see preparation of LDA above) was cooled to $-78{ }^{\circ} \mathrm{C}$ and added to the reaction flask via cannula transfer. The reaction mixture was allowed to warm to room temperature while stirring, then placed in a $45^{\circ} \mathrm{C}$ oil bath and left to stir under an atmosphere of $\mathrm{N}_{2}$ for 72 hours, during which time the reaction mixture turned brown and homogeneous. The reaction flask was opened to air and quickly transferred to a $1 \mathrm{~L}$ round bottom flask and volatiles were removed in vacuo on a rotary evaporator, resulting in a pale brown solid. $\mathrm{H}_{2} \mathrm{O}(400 \mathrm{~mL})$ was added to the flask under air and the solution was stirred rigorously for 15 minutes then filtered through a $60 \mathrm{~mL}$ medium-fritted glass funnel, resulting in a beige solid, which was washed with $\mathrm{H}_{2} \mathrm{O}(3 \times 50 \mathrm{~mL})$ and then dried in vacuo. Once dry, the beige solid was dissolved in EtOAc $(800 \mathrm{~mL})$ under air and filtered through a $60 \mathrm{~mL}$ medium-fritted glass funnel. The filtrate was collected, and volatiles were removed in vacuo on a rotary evaporator to produce a pale yellow solid. The pale yellow solid was washed with hexanes (3 x $40 \mathrm{~mL}$ ), resulting in a white solid. $6.3 \mathrm{~g} \mathrm{(31 \% ).}{ }^{1} \mathrm{H}$ NMR (400 MHz, DMSO-d 6 ) $\delta 3.56$ (br s, $\mathrm{Hz}, 16 \mathrm{H}), 2.87$ (br s, 16H). ${ }^{13} \mathrm{C}\left\{{ }^{1} \mathrm{H}\right\} \mathrm{NMR}\left(150 \mathrm{MHz}, \mathrm{DMSO}-d_{6}\right) \delta 133.3,67.3,50.7$. (HRMS) TOF MS ES ${ }^{+}(\mathrm{m} / \mathrm{z})[\mathrm{M}]^{+}$calculated for $\left[\mathrm{C}_{18} \mathrm{H}_{32} \mathrm{~N}_{4} \mathrm{O}_{4}\right]^{+}$368.2418; found 368.2409. Anal. Calcd. for $\mathrm{C}_{18} \mathrm{H}_{32} \mathrm{~N}_{4} \mathrm{O}_{4}$ : C, 58.67; H, 8.75; N, 15.20. Found: C, 58.74; H, 8.54; N, 15.19. 


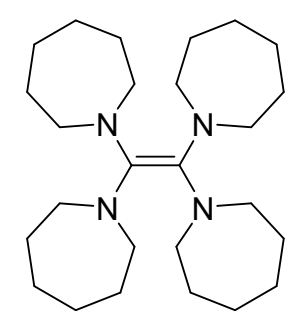

Tetrakis(azepano)ethylene (TAzE): A $500 \mathrm{~mL}$ round bottom flask was charged with a magnetic stir bar and azepan-1-ylmethylideneazepanium hexafluorophosphate (7.100 g, $20.0 \mathrm{mmol}, 1$ equiv.). The flask was dried in vacuo for 1 hour and then placed under an atmosphere of $\mathrm{N}_{2}$. Dry, degassed THF (14 mL) was added, and the flask was cooled to $-78^{\circ} \mathrm{C}$. LDA (20.0 mmol, 1 equiv.; see preparation of LDA above) was cooled to $-78{ }^{\circ} \mathrm{C}$ and added to the reaction flask via cannula transfer. The reaction mixture was allowed to warm to room temperature and left to stir under a positive pressure of $\mathrm{N}_{2}$ for 16 hours, during which time the reaction mixture turned homogeneous and pale brown. The reaction flask was opened to air and quickly transported to a rotary evaporator, where the volatiles were removed in vacuo, resulting in a sticky yellow solid. Hexanes $(75 \mathrm{~mL})$ was added to the resulting solid, and the mixture was filtered through a $60 \mathrm{~mL}$ medium-fritted glass funnel. The remaining contents of the round bottom flask and solids on the fritted funnel were washed with an additional $25 \mathrm{~mL}$ hexanes. The filtrate was transferred to a 500 $\mathrm{mL}$ round bottom flask and the volatiles were removed in vacuo on a rotary evaporator, resulting in a yellow oil. A magnetic stir bar was added to the round bottom flask, which was then fitted with a rubber septum. The flask was left under active vacuum for one hour and then backfilled with $\mathrm{N}_{2}$. Three 5-10 second cycles of vacuum followed by backfilling with $\mathrm{N}_{2}$ were then performed. Subsequently, degassed $\mathrm{EtOH}(25 \mathrm{~mL})$ was added to the flask via cannula transfer, and the contents stirred under a positive pressure of $\mathrm{N}_{2}$ until a fine white powder was suspended in a yellow solution (about 10 minutes). Degassed $\mathrm{H}_{2} \mathrm{O}(75 \mathrm{~mL})$ was added to the flask via cannula transfer, the rubber septum was removed, and the solution was filtered through a $60 \mathrm{~mL}$ medium-fritted glass funnel. The solid was washed with $\mathrm{H}_{2} \mathrm{O}(3 \times 30 \mathrm{~mL})$ and then quickly transferred to a glass scintillation vial and dried in vacuo. Once dry, the white solid product was stored in a glovebox under an $\mathrm{N}_{2}$ atmosphere, $1.6 \mathrm{~g}(39 \%) .{ }^{1} \mathrm{H} \mathrm{NMR}\left(500 \mathrm{MHz}, \mathrm{C}_{6} \mathrm{D}_{6}\right) \delta 3.13$ $(\mathrm{t}, \mathrm{J}=5.0 \mathrm{~Hz}, 16 \mathrm{H}), 1.67(\mathrm{~m}, 32 \mathrm{H}) .{ }^{13} \mathrm{C}\left\{{ }^{1} \mathrm{H}\right\} \mathrm{NMR}\left(125 \mathrm{MHz}, \mathrm{C}_{6} \mathrm{D}_{6}\right) \delta 136.7,52.6,30.4,28.7$. (HRMS) TOF MS ES $(\mathrm{m} / \mathrm{z})[\mathrm{M}]^{+}$calculated for $\left[\mathrm{C}_{26} \mathrm{H}_{48} \mathrm{~N}_{4}\right]^{+}$416.3873; found 416.3857. Anal. Calcd. for $\mathrm{C}_{26} \mathrm{H}_{48} \mathrm{~N}_{4}$ : C, 74.93; H, 11.61; N, 13.45. Found: C, 75.04; H, 11.72; N, 13.32. 


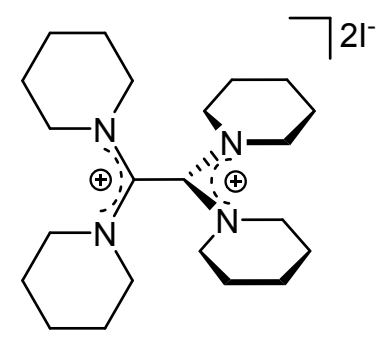

1,1'-(1,2-di(piperidin-1-yl)ethane-1,2-diylidene)bis(piperidin-1-ium) diiodide, [TPiE $]^{2+} 2[\mathrm{I}]^{-:}$ Under air, a solution of iodine $(0.0770 \mathrm{~g}, 0.306 \mathrm{mmol})$ in diethyl ether $(5 \mathrm{~mL})$ was prepared in a 2 dram vial and cooled to $0{ }^{\circ} \mathrm{C}$. Under $\mathrm{N}_{2}$, to a separate 2 dram vial was added a magnetic stir bar and TPiE $(0.100 \mathrm{~g}, 0.278 \mathrm{mmol})$, which was then cooled to $0{ }^{\circ} \mathrm{C}$. The 2 dram vial containing TPiE was opened to air and the diethyl ether solution of iodine was added quickly. The reaction was stirred for 10 minutes, during which time a yellow precipitate formed. The reaction was filtered through a $60 \mathrm{~mL}$ medium-fritted glass funnel. The resulting solid was rinsed with diethyl ether (3 $\mathrm{x} 10 \mathrm{~mL}$ ) and dried in vacuo to give a yellow solid, $0.1660 \mathrm{~g}(97 \%) .{ }^{1} \mathrm{H} \mathrm{NMR}\left(400 \mathrm{MHz}, \mathrm{CD}_{3} \mathrm{CN}\right)$ $\delta 3.88(\mathrm{t}, \mathrm{J}=5.69 \mathrm{~Hz}, 4 \mathrm{H}), 3.66(\mathrm{t}, \mathrm{J}=5.77 . \mathrm{Hz}, 4 \mathrm{H}), 1.89-1.71(\mathrm{~m}, 24 \mathrm{H}) .{ }^{13} \mathrm{C}\left\{{ }^{1} \mathrm{H}\right\} \mathrm{NMR}(100$ $\left.\mathrm{MHz}, \mathrm{CD}_{3} \mathrm{CN}\right) \delta 155.32,54.51,53.99,26.42,26.07,22.69$. (HRMS) TOF MS ES $(\mathrm{m} / \mathrm{z})[\mathrm{M}]^{2+}$ calculated for $\left[\mathrm{C}_{22} \mathrm{H}_{40} \mathrm{~N}_{4}\right]^{2+} 180.1621$; found 180.1622 . 


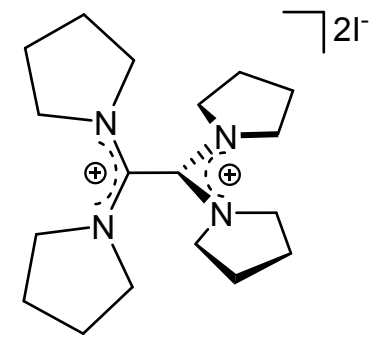

1,1'-(1,2-di(pyrrolidin-1-yl)ethane-1,2-diylidene)bis(pyrrolidin-1-ium) diiodide, [TPyE $]^{2+2}[\mathrm{I}]^{-:}$In a glovebox under an $\mathrm{N}_{2}$ atmosphere, a solution of iodine $(0.015 \mathrm{~g}, 0.059 \mathrm{mmol})$ in diethyl ether $(1.5$ $\mathrm{mL}$ ) was prepared in a 2-dram vial. To a separate 2 dram vial under an $\mathrm{N}_{2}$ atmosphere, a solution of TPyE (0.025 g, $0.082 \mathrm{mmol})$ in diethyl ether $(1.5 \mathrm{~mL})$ was prepared. The iodine solution was added dropwise to the TPyE solution with constant stirring over 5 minutes at room temperature, resulting in a white precipitate. Note: If the iodine solution was added too quickly or if iodine was added in excess, triiodide counterions were formed, resulting in a deep red precipitate. The reaction was removed from the glovebox and filtered through a $60 \mathrm{~mL}$ medium-fritted glass funnel. The resulting solid was rinsed with diethyl ether $(3 \times 10 \mathrm{~mL})$ and dried in vacuo to give a white solid, $0.020 \mathrm{~g}(61 \%) .{ }^{1} \mathrm{H}$ NMR (600 MHz, Methanol- $\left.d_{4}\right) \delta 4.31(\mathrm{t}, \mathrm{J}=6.81 \mathrm{~Hz}, 8 \mathrm{H}), 3.74(\mathrm{t}, \mathrm{J}=$ $6.51 \mathrm{~Hz}, 8 \mathrm{H}), 2.21-2.12(\mathrm{~m}, 16 \mathrm{H}) .{ }^{13} \mathrm{C}\left\{{ }^{1} \mathrm{H}\right\} \mathrm{NMR}\left(150 \mathrm{MHz}\right.$, Methanol- $\left.d_{4}\right) \delta 151.51,54.87,53.97$, 26.67, 25.41. (HRMS) TOF MS ES ${ }^{+}(\mathrm{m} / \mathrm{z})[\mathrm{M}]^{2+}$ calculated for $\left[\mathrm{C}_{18} \mathrm{H}_{32} \mathrm{~N}_{4}\right]^{2+} 152.1308$; found 152.1308 . 


\section{SV. Air Stability Studies of Tetraaminoethylenes}

Air Stability Studies Procedure:

A sample of tetraaminoethylene was prepared in a 2 dram vial inside a glovebox under an $\mathrm{N}_{2}$ atmosphere. If the sample was a solid, it was crushed up into a fine powder using the side of a spatula. The samples were removed from the glovebox and opened to air. The head space of the vial was sparged with air using a $20 \mathrm{~mL}$ syringe 5 times, then the sample was allowed to sit on the benchtop uncapped for an additional 5 minutes. A cap was then fitted to the vial and the sample was stored at room temperature. To take a time point, an aliquot of the sample was pumped into a glovebox and an NMR sample was prepared under a $\mathrm{N}_{2}$ atmosphere in a solvent where all species present in the sample were soluble to monitor decomposition using ${ }^{1} \mathrm{H}$ NMR spectroscopy (data for TDAE decomposition were collected in $\mathrm{C}_{6} \mathrm{D}_{6}$; data for TME, TPyE, and TAzE decomposition were collected in DMSO- $d_{6}$; data for TPiE decomposition were collected in 1:1 DMSO- $d_{6}: \mathrm{C}_{6} \mathrm{D}_{6}$ ). Decomposition is reported as integration of all unknown signals divided by integrations of all signals present in the spectrum. See Table 1 in the manuscript for percent decomposition results. 


\section{SVI. Solubility Studies of Tetraaminoethylenes}

\section{Solubility Studies Procedure:}

In a glovebox under an $\mathrm{N}_{2}$ atmosphere, a 2 dram vial with a PTFE seal cap was charged with a magnetic stir bar and a $0.0375 \mathrm{mmol}$ sample of tetraaminoethylene. The solid was ground to a fine powder inside the vial using the side of a spatula. $250 \mu \mathrm{L}$ of the appropriate solvent was added to the vial and the solution was allowed to stir at room temperature for 5 minutes. Vials which contained undissolved tetraaminoethylene were then heated to $80{ }^{\circ} \mathrm{C}$ in an aluminum heating block with a thermocouple and allowed to stir for five minutes.

Table S1. Solubility data for all reductants at $0.15 \mathrm{M}$ concentration. No $=$ not soluble at $80{ }^{\circ} \mathrm{C}, \mathrm{RT}=$ soluble at room temperature, $80^{\circ} \mathrm{C}=$ soluble at $80^{\circ} \mathrm{C}$. IPAc $=$ isopropyl acetate .

\begin{tabular}{ccccc}
\hline Solvent & TME & TPiE & TAzE & TPyE \\
\hline Pentane & No & RT & RT & RT \\
Benzene & RT & RT & RT & RT \\
1,4-Dioxane & RT & $80{ }^{\circ} \mathrm{C}$ & RT & RT \\
IPAc & $80{ }^{\circ} \mathrm{C}$ & $80{ }^{\circ} \mathrm{C}$ & RT & RT \\
2-MeTHF & $80{ }^{\circ} \mathrm{C}$ & RT & RT & RT \\
THF & RT & RT & RT & RT \\
DMAc & $80{ }^{\circ} \mathrm{C}$ & No & $80{ }^{\circ} \mathrm{C}$ & $80{ }^{\circ} \mathrm{C}$ \\
Acetonitrile & $80{ }^{\circ} \mathrm{C}$ & No & No & $80{ }^{\circ} \mathrm{C}$ \\
\hline
\end{tabular}


SVII. Procedure for ${ }^{1} \mathrm{H}$ NMR Yields of Products from Cross-Electrophile Coupling of Aryl and Alkyl Halides
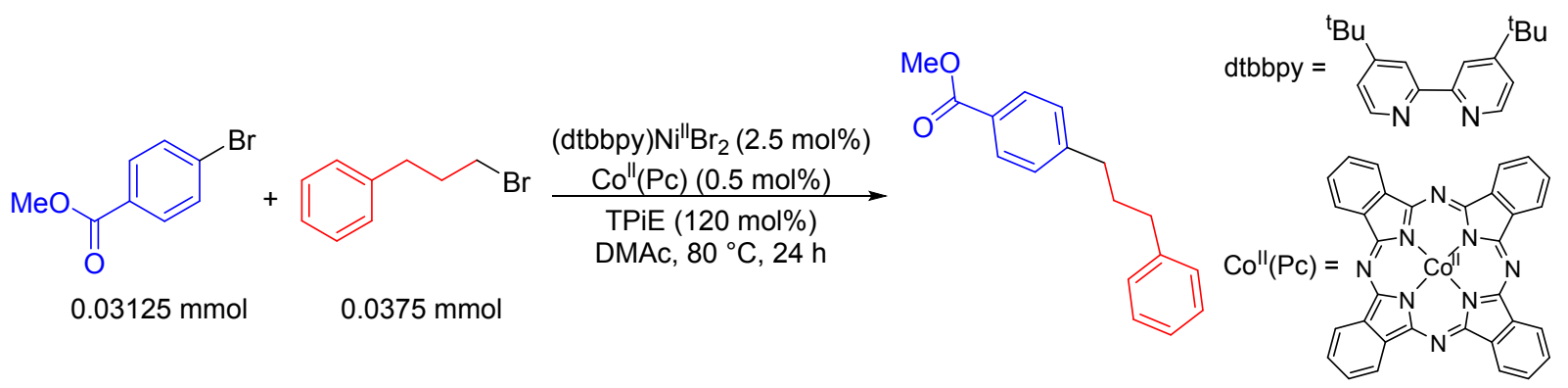

Figure S1. Cross-electrophile coupling of methyl 4-bromobenzoate and 1-bromo-3-phenylpropane.

Representative Procedure:

In a glovebox under an $\mathrm{N}_{2}$ atmosphere, methyl 4-bromobenzoate $(6.7 \mathrm{mg}, 0.03125 \mathrm{mmol})$ and TPiE (17.5 mg, $0.0375 \mathrm{mmol})$ were added to a 1 dram vial charged with a magnetic stir bar. A separate 2 dram vial was charged with $\mathrm{Co}{ }^{\mathrm{II}}(\mathrm{Pc})(1.8 \mathrm{mg}, 0.003125 \mathrm{mmol})$, (dtbbpy) $\mathrm{Ni}^{\mathrm{II}} \mathrm{Br}_{2}(7.6$ $\mathrm{mg}, 0.0156 \mathrm{mmol})$, 1-bromo-3-phenylpropane (114 $\mu \mathrm{L}, 0.75 \mathrm{mmol})$, and DMAc (5 $\mathrm{mL})$ to make a stock solution in a glovebox under an $\mathrm{N}_{2}$ atmosphere. $0.27 \mathrm{~mL}$ of the stock solution was added to the vial containing methyl 4-bromobenzoate and the reductant to initiate catalysis. The reaction was capped tightly with a PTFE seal cap and stirred at $80{ }^{\circ} \mathrm{C}$ for 24 hours. The reactions were opened to air and passed through a short plug of silica, which was washed with $5 \mathrm{~mL}$ EtOAc. The volatiles were removed, and the crude reaction mixture was dissolved in a solution of $\mathrm{CDCl}_{3}$ containing a known amount of hexamethylbenzene. Product yields were determined by integration of select peaks in the ${ }^{1} \mathrm{H}$ NMR spectrum against the hexamethylbenzene external standard. 


\section{SVIII. High Throughput Experimentation for Optimization of Drug-Like Aryl Halides}

Analytical Details for Analysis of High Throughput Experimentation:

UPLC/MS (ESI) was performed using a Waters Acquity UPLC I-Class system equipped with a binary pump, sample manager, column manager, sample organizer, a photodiode array detector, Single Quad Detector 2 with ESI source and MassLynx ${ }^{\circledR}$ software.

\section{Analytical Separations Method:}

Inject volume: $1 \mu \mathrm{L}$

Column Temperature: $45^{\circ} \mathrm{C}$

UV scan: $210-400 \mathrm{nM}$

CORTECS UPLC C18 $1.6 \mu \mathrm{M} 2.1 \times 50 \mathrm{~mm}$

Mobile Phase A: $0.1 \%$ TFA in Water

Mobile Phase B: $0.1 \%$ TFA in Acetonitrile

Details of Elution

\begin{tabular}{|c|c|c|c|}
\hline Time (min) & Flow (mL/min) & \% A & $\% \mathrm{~B}$ \\
\hline 0.00 & 0.700 & 95 & 5 \\
\hline 1.70 & 0.700 & 0 & 100 \\
\hline 1.95 & 0.700 & 0 & 100 \\
\hline 1.96 & 0.700 & 95 & 100 \\
\hline 2.00 & 0.700 & 95 & 5 \\
\hline
\end{tabular}

Hardware for High Throughput Experimentation:

The reactions were performed in a 96 well reaction block (Analytical Sales \& Services, Inc. catalog \# 96960) using $1 \mathrm{~mL}$ reaction vials (Analytical Sales \& Services, Inc. catalog \# 884001), a PFA sheet (Analytical Sales \& Services, Inc. catalog \#: 96967) and rubber mat (Analytical Sales \& Services, Inc. catalog \#: 96965) for sealing the block, and 96 parylene coated stir dowels (1.98mm diameter, $4.80 \mathrm{~mm}$ length, V\&P Scientific, Inc. product \# VP 711D-1) for stirring. The reaction block was stirred using a tumble stirrer (tumble stirrer: V\&P Scientific, Inc. Model \# VP710 S) and heating was applied using a heating jacket (V\&P Scientific, Inc. Model VP 
741ABZ-R-MB). The analytical samples were filtered through a 0.45 um filter plate (Millipore Sigma, Part\# MSRLN0450).

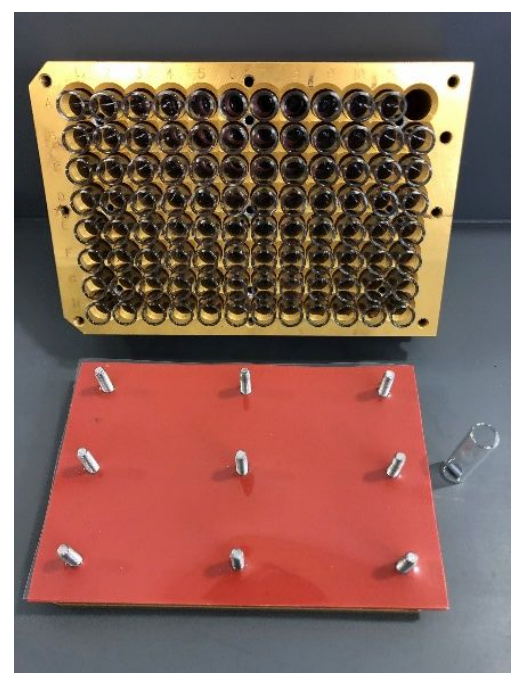

Figure S2. Representative image of a reaction block, reaction vessels, stir bar, PFA sheet, and rubber mat used in high throughput experimentation.

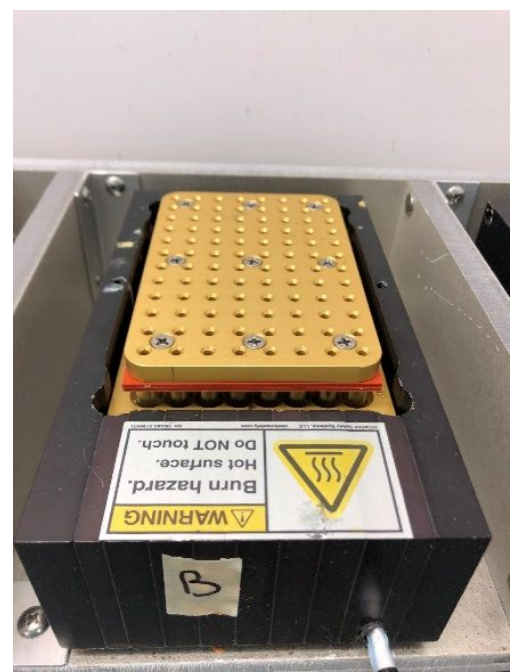

Figure S3. Representative image of a reaction block inside a heating jacket on a tumble stirrer used in high throughput experimentation.

Reaction Components Used in High Through Put Experimentation:

All coupling partners, catalysts, and reductants were dosed as mixtures in DMAc inside a nitrogen filled glovebox. If the mixture in DMAc was not soluble, the mixture was dosed while it was stirred. The DMAc that was used in all experiments was purchased from Millipore Sigma in an air free, sure seal bottle, and used as is, after opening inside a nitrogen filled glove box. Aryl and alkyl halide DMAc mixtures were prepared by weighing the aryl and alkyl halides into 
respective dram vials open to air (each with a stir bar), then bringing the vials inside a nitrogen filled glove box and adding DMAc. The mixtures of CoPc and organic reductant were prepared by weighing CoPc and organic reductant into respective dram vials (each with a stir bar) inside a nitrogen filled glovebox, then adding DMAc. The mixture of (dtbbpy) $\mathrm{Ni}^{\mathrm{II}} \mathrm{Br}_{2}$ was prepared by weighing $\mathrm{Ni}^{\mathrm{II}} \mathrm{Br}_{2}$ (1 equiv.) and dtbbpy (1 equiv.) in a dram vial (with a stir bar) inside a nitrogen filled glove box followed by adding DMAc. The (dtbbpy) $\mathrm{Ni}^{\mathrm{II}} \mathrm{Br}_{2} \mathrm{DMAc}$ mixture was allowed to stir for $20 \mathrm{~min}$ at $25^{\circ} \mathrm{C}$ before use. The concentration of the mixtures of each reaction component in DMAc is shown below.

General Procedure for High Throughput Cross-Electrophile Coupling Using Dual Ni-CoCatalysis with Organic Reductants:

Each reaction component was added as a stock mixture using DMAc as solvent. Using Eppendorf pipettes in a nitrogen filled glove box, each $1 \mathrm{~mL}$ reaction vial (containing a parylene coated stir dowel) was charged with an aliquot of stock mixture of aryl halide (14 uL, 7 umol, 1 equiv, $0.5 \mathrm{M}$ ), 1-Br-3-Ph-Propane (22 ul, 11 umol, 1.6 equiv., $0.5 \mathrm{M})$, (dtbbpy) $\mathrm{Ni}^{\mathrm{II}} \mathrm{Br}_{2}(14 \mathrm{uL}, 0.35$ umol, 0.05 equiv., $0.025 \mathrm{M}$, for 5 mol\%, volumes scaled appropriately for other loadings), CoPc $(14 \mathrm{uL}, 0.35$ umol, 0.05 equiv, $0.025 \mathrm{M}$, for 5 mol\% loading, volumes scaled appropriately for other loadings), and organic reductant (18 uL, 11 umol, 1.6 equiv., 0.6 M). DMAc was added to vials, where required, to reach a final concentration of $0.1 \mathrm{M}$. The reaction plate was then sealed and placed in a preheated $\left(80^{\circ} \mathrm{C}\right)$ tumble stirrer. The reaction block was stirred at $80{ }^{\circ} \mathrm{C}$ for 18 hours. At this time, the reaction block was allowed to cool to $25^{\circ} \mathrm{C}$ and removed from the tumble stirrer and glove box. The plate was centrifuged, opened to air and $4 \mathrm{uL}$ of the crude mixture was diluted in $200 \mathrm{uL}$ DMSO. These mixtures then were forced through a $0.45 \mathrm{uM}$ filter plate (Millipore Sigma part\# MSRLN0450). These mixtures were injected into the UPLC/MS for analytical analysis. The yield of the reaction under optimal conditions, as judged by Liquid Chromatography Area Percent (LCAP) at $254 \mathrm{~nm}$, was then measured using NMR spectroscopy (see section SIX). 


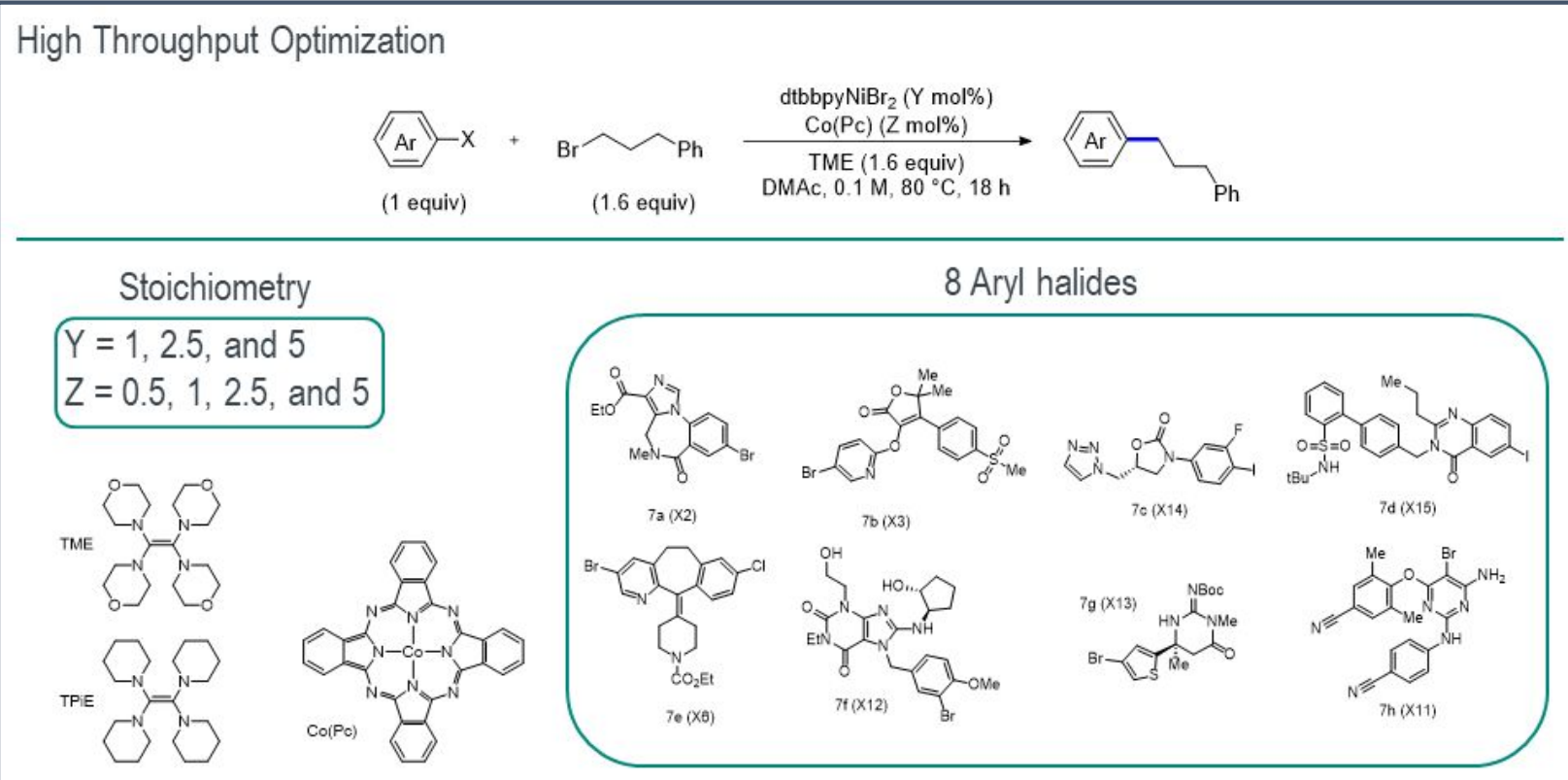

$(8 \text { Aryl halides })^{\star}(3 \text { Ni equiv })^{\star}(4 \text { Co equiv })^{\star}(2$ Reductants $)=192$ unique reactions

Figure S4. High throughput optimization of Ni/Co-catalyzed cross-electrophile reductive coupling promoted by organic reductants TME and TPiE.

\begin{tabular}{|c|c|c|c|c|c|c|c|c|c|c|c|c|c|}
\hline $\mathrm{Ni}(\mathrm{mol} \%)$ & & & & & & & & & & & & & \\
\hline Co (mol\%) & 0.5 & 1 & 2.5 & 5 & 0.5 & 1 & 2.5 & 5 & 0.5 & 1 & 2.5 & 5 & \\
\hline Column & 1 & 2 & 3 & 4 & 5 & 6 & 7 & 8 & 9 & 10 & 11 & 12 & $\operatorname{ArX}$ \\
\hline Row A & & & & 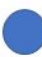 & 0 & & 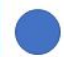 & & 0 & & & 0 & $7 a$ \\
\hline B & & & & 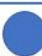 & 0 & & 0 & & 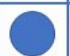 & & & D & $7 \mathrm{~b}$ \\
\hline C & & & 0 & 0 & 0 & 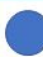 & 0 & & 0 & 0 & 0 & 0 & 7c \\
\hline D & & 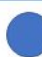 & 0 & 0 & 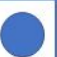 & 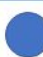 & 0 & & 0 & & D & 0 & $7 d$ \\
\hline $\mathrm{E}$ & & & & 0 & 0 & & 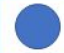 & & 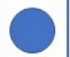 & & & D & $7 e$ \\
\hline$F$ & & & ) & 0 & 0 & & C & & 0 & 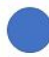 & 0 & 0 & $7 f$ \\
\hline G & 0 & & 0 & 0 & 0 & & 0 & & 0 & 2 & 0 & 0 & $7 \mathrm{~g}$ \\
\hline $\mathrm{H}$ & & & & ก & O & & 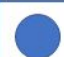 & & 0 & ก & 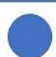 & 0 & $7 \mathrm{~h}$ \\
\hline
\end{tabular}

Figure S5. Reaction array schematic used for high throughput optimization of the cross-electrophile coupling of aryl halides and 1-Br-3-Ph-propane promoted by TME and TPiE. 


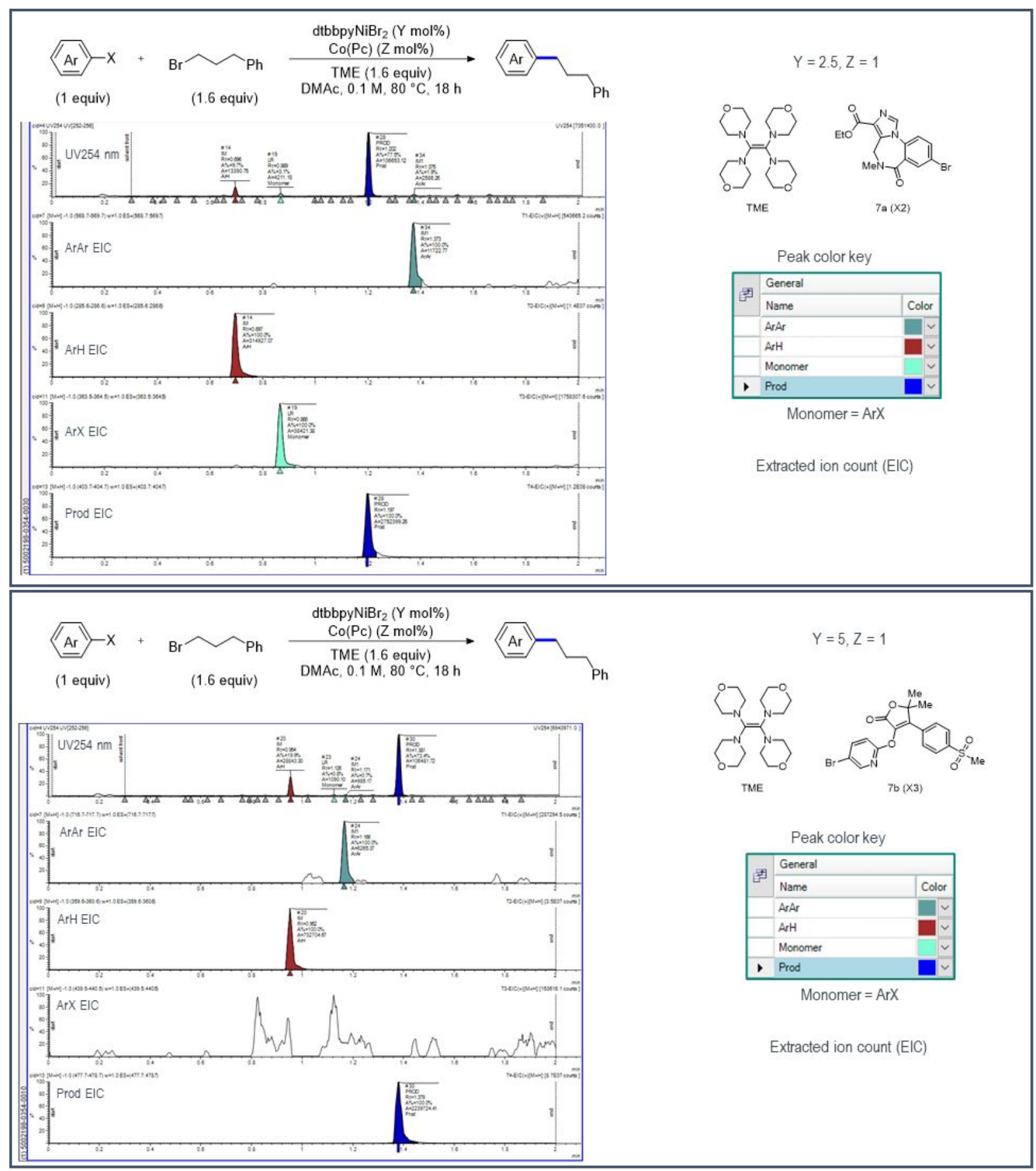




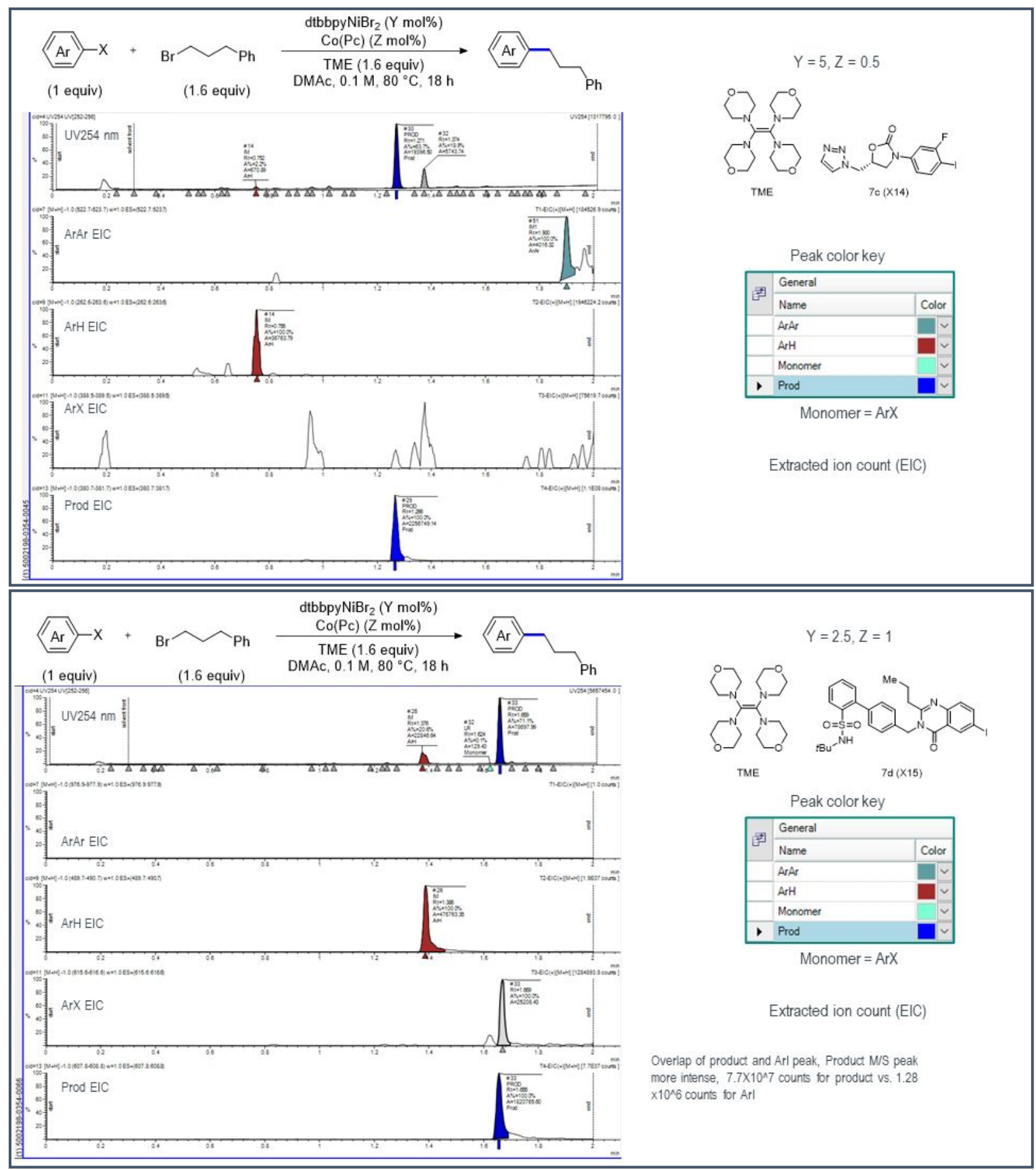




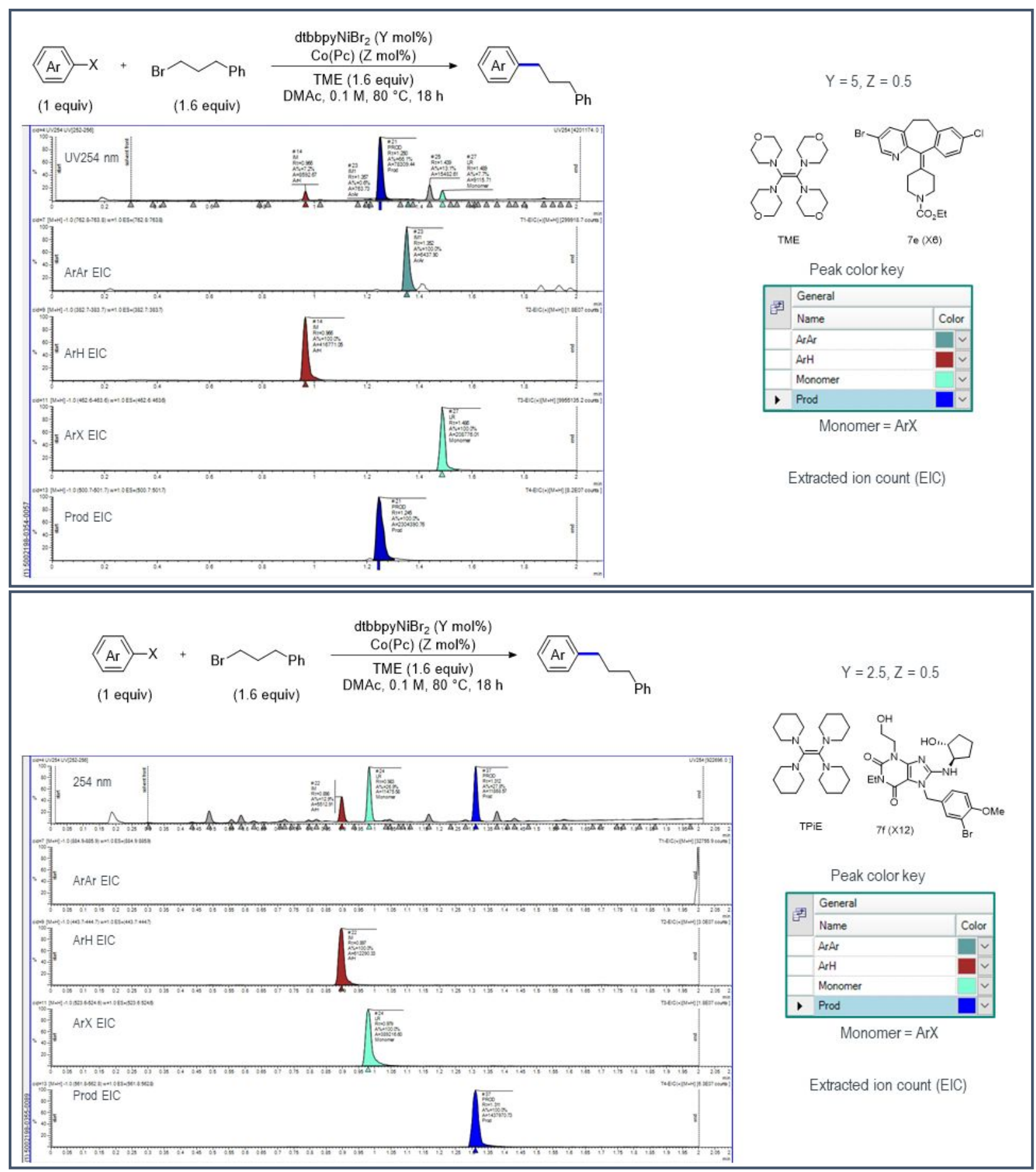




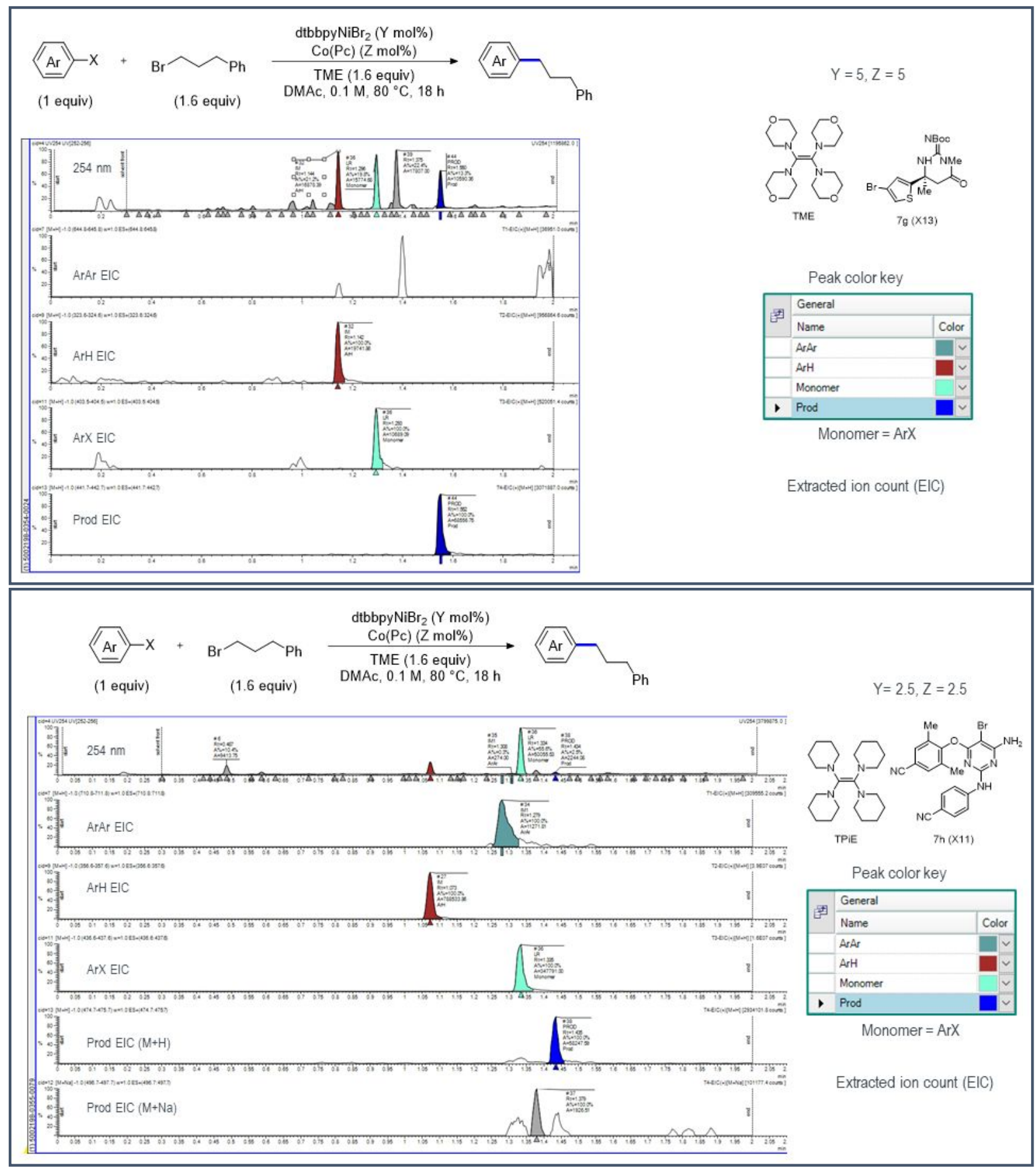

Figure S6. Representative examples of raw data from high throughput optimization experiments. 


\section{SIX. Procedure for ${ }^{1} \mathrm{H}$ NMR Yields of Cross-Electrophile Coupling of Drug-Like Aryl Halides and Alkyl Halides}

Procedure:

See section SVII for representative experimental setup and workup, but the reactions were performed on a $0.03 \mathrm{mmol}$ scale of the aryl electrophile with $0.048 \mathrm{mmol}$ (1.6 equiv.) of the alkyl electrophile at $0.1 \mathrm{M}$ concentration for 18 hours (other reagents scaled linearly). The silica plug was washed with $10 \mathrm{~mL}$ EtOAc instead of $5 \mathrm{~mL}$ during workup. For individual reactions with TPiE and TME, see Figure 7 in the manuscript. See Figure S7 below for reactions with TDAE. Note: The conditions for TDAE were not optimized using HTE. Instead, for each substrate, the optimized conditions for TPiE or TME that required higher catalyst loadings were used with $T D A E$. The results are broadly comparable with the only exception being $\mathbf{7 b}$ where TDAE gave a significantly lower yield. Further optimization was not attempted. The ${ }^{1} \mathrm{H}$ NMR spectra of the compounds matched those previously reported. ${ }^{8}$ 


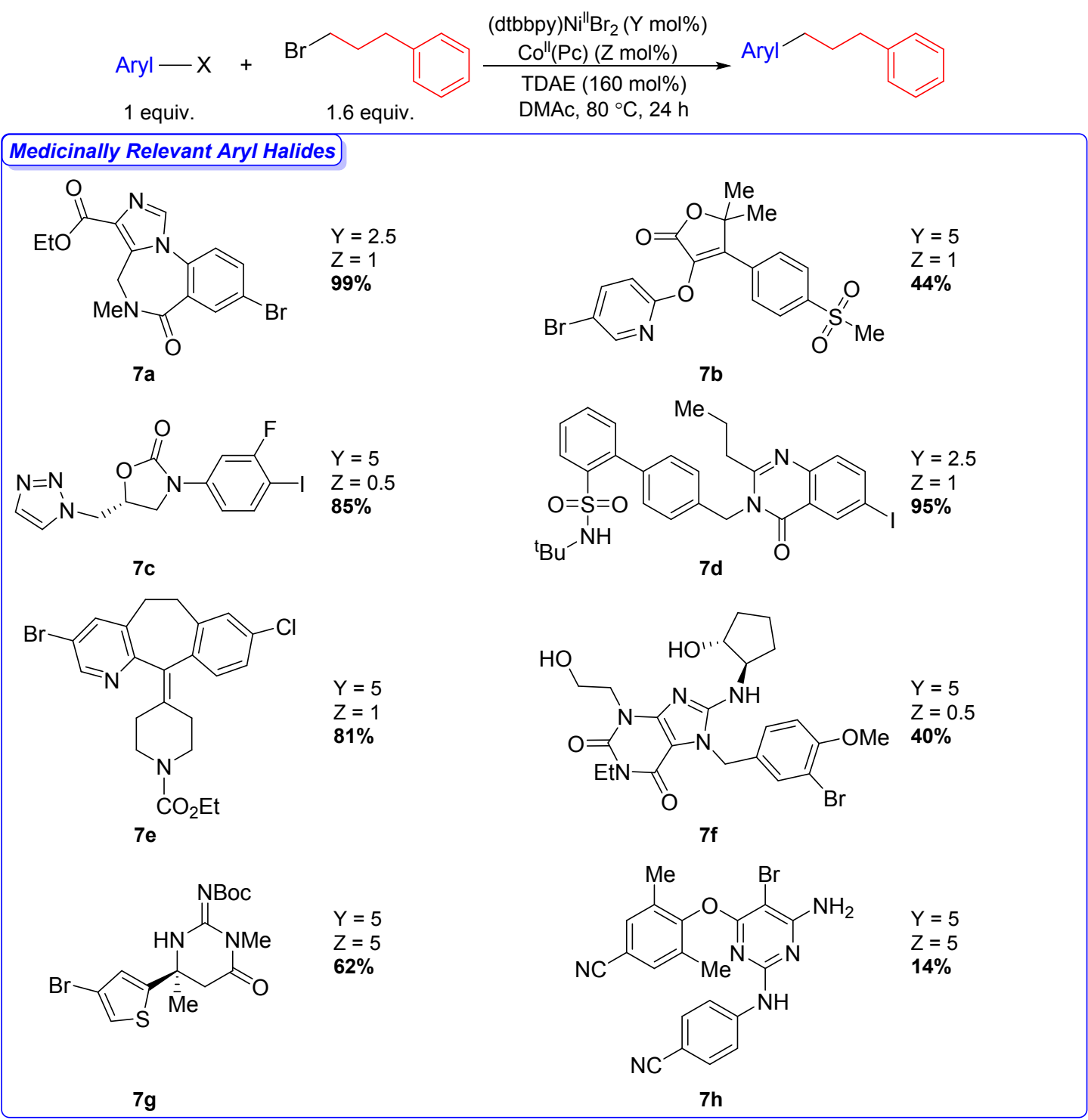

Figure S7. ${ }^{1} \mathrm{H}$ NMR yields of products from cross-electrophile coupling of drug-like aryl halides with 1bromo-3-phenylpropane using TDAE as the reductant. 


\section{SX. Reductant Screen for Intermolecular Three-Component Dicarbofunctionalization of}

\section{Alkenes}

To further establish that our reductants can be used as direct and more practical replacements for TDAE, we looked at another example of a CEC reaction that uses TDAE as the electron source. The intermolecular three-component dicarbofunctionalization of alkenes ${ }^{9}$ reported by Nevado and co-workers is an efficient pathway to construct complex molecules through the simultaneous formation of two vicinal $\mathrm{C}-\mathrm{C}$ bonds by coupling alkenes with alkyl and aryl iodides (Figure S8). Using the representative procedure described below, we demonstrated that TPyE gives comparable performance to TDAE for this reaction, under essentially the optimized conditions for TDAE (Table S2). The only change was the use of a higher temperature, $60{ }^{\circ} \mathrm{C}$, to promote solubility. TPiE and TAzE also give significant yields of product, but only a small amount of product was observed with TME. It is likely that with further optimization, higher yields could be achieved with our reductants, but overall, this result reinforces that our reductants can be used as practical replacements for TDAE with, in some cases, essentially no optimization.
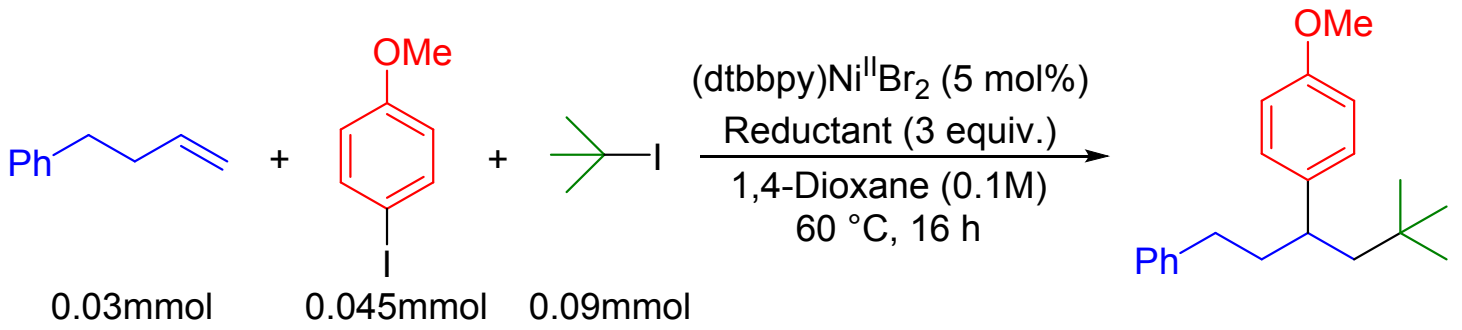

Figure S8. Intermolecular three-component dicarbofunctionalization of 4-phenyl-1-butene, 4iodoanisole, and tert-butyl iodide.

Table S2. NMR yields of intermolecular three-component dicarbofunctionalization between 4-iodoanisole, 4-phenyl-1-butene, and tert-butyl iodide with the new reductants.

\begin{tabular}{ccc}
\hline Reductant & $\mathbf{E}^{\mathbf{0}}\left(\mathbf{V}\right.$ vs. $\left.\mathbf{F c} / \mathbf{F c}^{+}\right)$ & Product Yield (\%) \\
\hline TME & -0.85 & 9 \\
TPiE & -1.06 & 31 \\
TAzE & -1.09 & 25 \\
TDAE & -1.11 & 62 \\
TPyE & -1.32 & 51 \\
\hline
\end{tabular}

Representative Procedure: 
The procedure was adapted from a literature report. ${ }^{9 b}$ In a glovebox under an $\mathrm{N}_{2}$ atmosphere, 4-iodoanisole $(10.5 \mathrm{mg}, 0.045 \mathrm{mmol})$ and the reductant $(0.09 \mathrm{mmol})$ were added to a 1 dram vial charged with a magnetic stir bar. A separate 2 dram vial was charged with (dtbbpy) $\mathrm{Ni}^{\mathrm{II}} \mathrm{Br}_{2}$ (3 mg, $\left.0.006 \mathrm{mmol}\right)$, 4-phenyl-1-butene (18 $\left.\mu \mathrm{L}, 0.12 \mathrm{mmol}\right)$, tert-butyl iodide (43 $\mu \mathrm{L}, 0.36 \mathrm{mmol})$, and 1,4-dioxane $(1.2 \mathrm{~mL})$ to make a stock solution in a glovebox under an $\mathrm{N}_{2}$ atmosphere. $0.31 \mathrm{~mL}$ of the stock solution was added to the vial containing 4-iodoanisole and the reductant to initiate catalysis. The reaction was capped tightly with a PTFE seal cap and stirred at $60{ }^{\circ} \mathrm{C}$ for 16 hours. The reaction was opened to air and passed through a short plug of silica, which was washed with $5 \mathrm{~mL}$ EtOAc. The volatiles were removed, and the crude reaction mixture was dissolved in a solution of $\mathrm{CDCl}_{3}$ containing a known amount of hexamethylbenzene. Product yields were determined by integration of select peaks in the ${ }^{1} \mathrm{H}$ NMR spectrum against the hexamethylbenzene external standard. 


\section{SXI. Synthesis of Katritzky Salts}

General Procedure B:

The procedure was adapted from a literature report. ${ }^{11}$ To a 6 dram vial equipped with a magnetic stir bar was added the pyrylium cation (1 equiv.) and activated $3 \AA$ molecular sieves $(0.5 \mathrm{~g}$ per mmol of pyrylium cation) in a glovebox under an $\mathrm{N}_{2}$ atmosphere. The vial was capped tightly with a PTFE seal cap and brought out of the glovebox. A vent needle was added to the vial, and dry, degassed DCM (0.5 M), $\mathrm{NEt}_{3}$ (not dried or degassed) (2 equiv.), and amine (not dried or degassed) (1.2 equiv.) were added via syringes in that order. The vent needle was removed, and the reaction was allowed to stir for $20 \mathrm{~min}$ at room temperature. A vent needle was reinserted, then glacial acetic acid (2 equiv.) and a second portion of dry, degassed DCM (0.25 M) were added to the reaction via syringes in that order. The vent needle was removed, and the reaction was allowed to stir overnight at room temperature. The reaction was opened to air and filtered through a short pad of celite, and the vial and celite were rinsed with a small portion of DCM. The filtrate was collected and washed with $1 \mathrm{M} \mathrm{HCl}\left(4 \times 8 \mathrm{~mL}\right.$ per mmol of pyrylium cation), sat. $\mathrm{NaHCO}_{3}(4 \times 8$ $\mathrm{mL}$ per mmol of pyrylium cation), and brine ( $1 \times 8 \mathrm{~mL}$ per mmol of pyrylium cation). The organic layer was dried over $\mathrm{MgSO}_{4}$, filtered, and concentrated in vacuo. The resulting solid was triturated in $\mathrm{EtOH}$ for $20 \mathrm{~min}$, filtered, triturated in EtOAc for $20 \mathrm{~min}$, filtered, triturated again in diethyl ether for $20 \mathrm{~min}$, and filtered. The resulting solid was dried in vacuo overnight. If no precipitate is formed upon trituration in EtOH, the crude product was purified by silica gel column chromatography using acetone:DCM solvent mixtures. Note: The Katritzky salt product is mildly soluble in both EtOH and EtOAc, so it is important to use minimal amount of solvent during trituration to avoid loss of product during filtration. In cases where a significant amount of product remained dissolved, the filtrate was recovered and concentrated in vacuo, and the resulting solid was triturated in the same order as described above with less amount of solvent. 
SXII. Characterization of Katritzky Salts

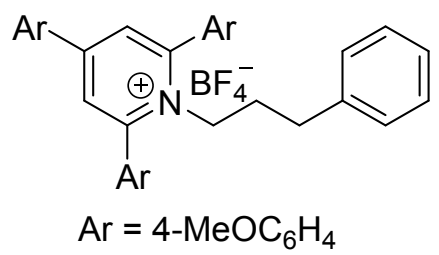

2,4,6-tris(4-methoxyphenyl)-1-(3-phenylpropyl)pyridin-1-ium tetrafluoroborate (1): The procedure was adapted from a literature report. ${ }^{10}$ To a 2 dram vial equipped with a magnetic stir bar in a glove box under an $\mathrm{N}_{2}$ atmosphere was added ethanol (1.25 mL, $\left.1.0 \mathrm{M}\right), 2,4,6$ tris(trimethoxyphenyl)pyrylium tetrafluoroborate $(500 \mathrm{mg}, 1.0 \mathrm{mmol})$ and 3-phenyl-1propylamine $(0.18 \mathrm{~mL}, 1.2 \mathrm{mmol})$. The reaction was capped tightly with a PTFE seal cap, removed from the glove box, and heated at $80{ }^{\circ} \mathrm{C}$ with stirring for 4 hours. The reaction was allowed to cool to room temperature, and volatiles were removed in vacuo. The crude substrate was purified by silica gel column chromatography using 15:85 acetone:DCM solvent mixtures. Pale yellow solid, $0.520 \mathrm{~g}(86 \%) .{ }^{1} \mathrm{H}$ NMR (400 MHz, $\left.\mathrm{CDCl}_{3}\right) \delta 7.71(\mathrm{~m}, 4 \mathrm{H}), 7.62(\mathrm{~d}, \mathrm{~J}=8.73 \mathrm{~Hz}, 4 \mathrm{H}), 7.07(\mathrm{~m}$, 3H), $7.01(\mathrm{~m}, 6 \mathrm{H}), 6.68(\mathrm{~m}, 2 \mathrm{H}), 4.45(\mathrm{~m}, 2 \mathrm{H}), 3.88(\mathrm{~s}, 6 \mathrm{H}), 3.85(\mathrm{~s}, 3 \mathrm{H}), 2.13$. (t, J = 6.97 Hz, 2H), $1.68(\mathrm{~m}, 2 \mathrm{H}) .{ }^{13} \mathrm{C}\left\{{ }^{1} \mathrm{H}\right\} \mathrm{NMR}\left(100 \mathrm{MHz}, \mathrm{CDCl}_{3}\right) \delta 163.27,161.53,156.53,154.60,139.03$, $130.66,129.92,128.54,128.01,126.24,125.88,125.32,125.01,115.37,114.85,55.77,55.62$, 53.95, 32.40, 30.85. ${ }^{19} \mathrm{~F}$ NMR (376 $\left.\mathrm{MHz} \mathrm{CDCl}_{3}\right) \delta-153.21$ (minor, $\left.{ }^{10} \mathrm{BF} 4\right),-153.26$ (major, $\left.{ }^{11} \mathrm{BF} 4\right)$. (HRMS) TOF MS ES ${ }^{+}(\mathrm{m} / \mathrm{z})[\mathrm{M}]^{+}$calculated for $\left[\mathrm{C}_{35} \mathrm{H}_{34} \mathrm{NO}_{3}\right]^{+}$516.2533; found 516.2537.

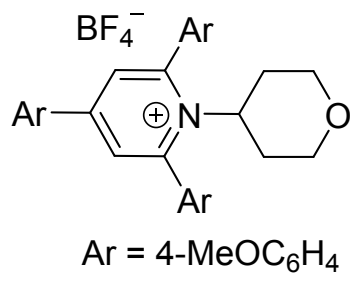

2,4,6-tris(4-methoxyphenyl)-1-(tetrahydro-2H-pyran-4-yl)pyridin-1-ium tetrafluoroborate (2): Synthesized according to general procedure B on a $1.4 \mathrm{mmol}$ scale of 2,4,6-tris(4methoxyphenyl)pyrylium tetrafluoroborate. Pale yellow solid, $0.630 \mathrm{~g}(76 \%) .{ }^{1} \mathrm{H}$ NMR (600 MHz, $\left.\mathrm{CDCl}_{3}\right) \delta 7.74(\mathrm{~m}, 4 \mathrm{H}), 7.67(\mathrm{~d}, \mathrm{~J}=8.73 \mathrm{~Hz}, 4 \mathrm{H}), 7.09(\mathrm{~d}, \mathrm{~J}=8.75 \mathrm{~Hz}, 4 \mathrm{H}), 6.99(\mathrm{~d}, \mathrm{~J}=8.95 \mathrm{~Hz}$ 2H), $4.88(\mathrm{tt}, \mathrm{J}=12.26,3.10 \mathrm{~Hz}, 1 \mathrm{H}), 3.91(\mathrm{~s}, 6 \mathrm{H}), 3.86(\mathrm{~s}, 3 \mathrm{H}), 3.76(\mathrm{dd}, \mathrm{J}=11.54,3.76 \mathrm{~Hz}, 2 \mathrm{H})$, $2.89(\mathrm{t}, \mathrm{J}=11.36 \mathrm{~Hz}, 2 \mathrm{H}), 2.05(\mathrm{qd}, \mathrm{J}=12.07,3.94 \mathrm{~Hz}, 2 \mathrm{H}) .{ }^{13} \mathrm{C}\left\{{ }^{1} \mathrm{H}\right\} \mathrm{NMR}\left(150 \mathrm{MHz}, \mathrm{CDCl}_{3}\right) \delta$ 
163.36, 161.62, 157.48, 154.22, 131.15, 130.15, 127.09, 126.16, 125.84, 115.36, 114.53, 68.83, 68.05, 55.77, 55.70, 34.14. ${ }^{19} \mathrm{~F}\left(376 \mathrm{MHz}, \mathrm{CDCl}_{3}\right) \delta-153.16$ (minor, $\left.{ }^{10} \mathrm{BF} 4\right),-153.22$ (major, $\left.{ }^{11} \mathrm{BF} 4\right)$. (HRMS) TOF MS ES ${ }^{+}(\mathrm{m} / \mathrm{z})[\mathrm{M}]^{+}$calculated for $\left[\mathrm{C}_{31} \mathrm{H}_{32} \mathrm{NO}_{4}\right]^{+} 482.2326$; found 482.2314 .

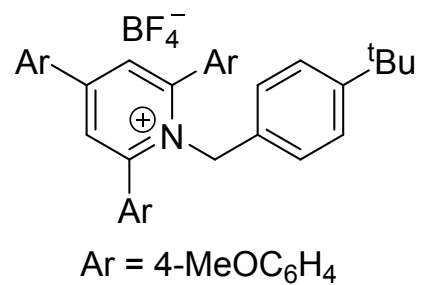

1-(4-(tert-butyl)benzyl)-2,4,6-tris(4-methoxyphenyl)pyridin-1-ium tetrafluoroborate (3): Synthesized according to general procedure B on a 1.6 mmol scale of 2,4,6-tris(4methoxyphenyl)pyrylium tetrafluoroborate. White solid, $0.590 \mathrm{~g}(57 \%) .{ }^{1} \mathrm{H}$ NMR (400 MHz, $\left.\mathrm{CDCl}_{3}\right) \delta 7.77(\mathrm{~m}, 4 \mathrm{H}), 7.61(\mathrm{~d}, \mathrm{~J}=7.67 \mathrm{~Hz}, 4 \mathrm{H}), 7.05(\mathrm{~m}, 4 \mathrm{H}), 6.96(\mathrm{~d}, \mathrm{~J}=8.92 \mathrm{~Hz}, 4 \mathrm{H}), 6.33(\mathrm{~d}$, $\mathrm{J}=8.34 \mathrm{~Hz}, 2 \mathrm{H}), 5.77(\mathrm{~s}, 2 \mathrm{H}), 3.87(\mathrm{~s}, 3 \mathrm{H}), 3.83(\mathrm{~s}, 6 \mathrm{H}), 1.20(\mathrm{~s}, 9 \mathrm{H}) .{ }^{13} \mathrm{C}\left\{{ }^{1} \mathrm{H}\right\} \mathrm{NMR}(100 \mathrm{MHz}$, $\left.\mathrm{CDCl}_{3}\right) \delta 163.46,161.58,157.64,154.73,151.46,131.75,131.69,129.96,126.32,125.68,125.64$, $125.54,124.81,115.54,114.70,58.27,55.81,55.65,34.61,31.29 .{ }^{19} \mathrm{~F} \mathrm{NMR}\left(376 \mathrm{MHz}, \mathrm{CDCl}_{3}\right) \delta$ -152.94 (minor, $\left.{ }^{10} \mathrm{BF} 4\right),-153.00$ (major, $\left.{ }^{11} \mathrm{BF} 4\right)$. (HRMS) TOF $\mathrm{MS} \mathrm{ES}^{+}(\mathrm{m} / \mathrm{z})[\mathrm{M}]^{+}$calculated for $\left[\mathrm{C}_{37} \mathrm{H}_{38} \mathrm{NO}_{3}\right]^{+}$542.2846; found 542.2836.

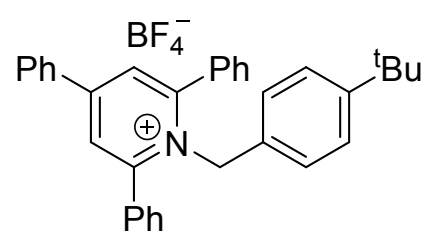

1-(4-(tert-butyl)benzyl)-2,4,6-triphenylpyridin-1-ium tetrafluoroborate (4): Synthesized according to modified general procedure B. A $250 \mathrm{~mL}$ Schlenk flask was removed from an oven at $130{ }^{\circ} \mathrm{C}$ and was charged with 2,4,6-triphenylpyrylium tetrafluoroborate (5.1 g, $\left.12.6 \mathrm{mmol}\right), 3 \AA$ molecular sieves $(6.4 \mathrm{~g})$, and a magnetic stir bar. The content of the Schlenk flask was dried in vacuo for 30 min and was put under a positive pressure of $\mathrm{N}_{2}$ using standard Schlenk line techniques. The Schlenk flask was fitted with a rubber septum, and dry, degassed DCM (25 mL, $0.5 \mathrm{M}), \mathrm{NEt}_{3}$ (not dried or degassed) (3.6 mL), and 4-tert-butylbenzylamine (not dried or degassed) $(2.6 \mathrm{~mL})$ were added via syringes in that order. The reaction was stirred at room temperature for $20 \mathrm{~min}$. Glacial acetic acid $(1.5 \mathrm{~mL})$ and a second portion of dry, degassed DCM (25 mL, $0.25 \mathrm{M})$ 
were added to the reaction via syringes in that order. Under a positive pressure of $\mathrm{N}_{2}$, the rubber septum was then replaced with a glass stopper, and the reaction was allowed to stir overnight at room temperature. See general procedure B for workup. White solid, $4.320 \mathrm{~g}(62 \%) .{ }^{1} \mathrm{H}$ NMR (400 $\left.\mathrm{MHz} \mathrm{CDCl}_{3}\right) \delta 7.93(\mathrm{~s}, 2 \mathrm{H}), 7.80(\mathrm{~d}, \mathrm{~J}=8.00 \mathrm{~Hz}, 2 \mathrm{H}), 7.63(\mathrm{~d}, \mathrm{~J}=7.29 \mathrm{~Hz}, 4 \mathrm{H}), 7.58-7.43(\mathrm{~m}$, 9H), $7.09(\mathrm{~d}, \mathrm{~J}=8.38 \mathrm{~Hz}, 2 \mathrm{H}), 6.36(\mathrm{~d}, \mathrm{~J}=8.29 \mathrm{~Hz}, 2 \mathrm{H}), 5.74(\mathrm{~s}, 2 \mathrm{H}), 1.22(\mathrm{~s}, 9 \mathrm{H})$. The ${ }^{1} \mathrm{H}$ NMR data are consistent with a previous literature report. ${ }^{12}$<smiles></smiles>

1-(4-methoxybenzyl)-2,4,6-triphenylpyridin-1-ium tetrafluoroborate (5): Synthesized according to general procedure $\mathrm{B}$ on a $2.5 \mathrm{mmol}$ scale of 2,4,6-triphenylpyrylium tetrafluoroborate. White solid, $0.650 \mathrm{~g}(50 \%) .{ }^{1} \mathrm{H} \mathrm{NMR}\left(400 \mathrm{MHz}, \mathrm{CDCl}_{3}\right) \delta 7.92(\mathrm{~s}, 2 \mathrm{H}), 7.80(\mathrm{~d}$, $\mathrm{J}=6.91 \mathrm{~Hz}, 2 \mathrm{H}), 7.66(\mathrm{~d}, \mathrm{~J}=6.95 \mathrm{~Hz}, 4 \mathrm{H}), 7.58-7.46(\mathrm{~m}, 9 \mathrm{H}), 6.60(\mathrm{~d}, \mathrm{~J}=8.39 \mathrm{~Hz}, 2 \mathrm{H}), 6.35$ (d, $\mathrm{J}=8.32 \mathrm{~Hz}, 2 \mathrm{H}), 5.71(\mathrm{~s}, 2 \mathrm{H}), 3.71(\mathrm{~s}, 3 \mathrm{H})$. The ${ }^{1} \mathrm{H}$ NMR data are consistent with a previous literature report. ${ }^{13}$

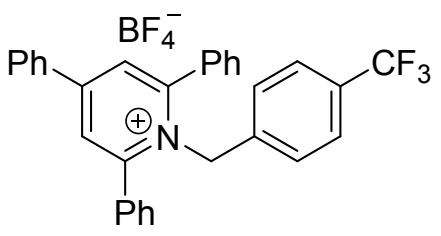

\section{2,4,6-triphenyl-1-(4-(trifluoromethyl)benzyl)pyridin-1-ium}

Synthesized according to general procedure B on a $2.5 \mathrm{mmol}$ scale of 2,4,6-triphenylpyrylium tetrafluoroborate. No precipitate formed upon trituration in $\mathrm{EtOH}$, and the crude product was purified by silica gel column chromatography using 5:95 acetone:DCM solvent mixtures. The product was triturated in $5 \mathrm{~mL}$ diethyl ether overnight after the column, filtered, and dried in vacuo. White solid, $0.850 \mathrm{~g}(62 \%) .{ }^{1} \mathrm{H} \mathrm{NMR}\left(400 \mathrm{MHz}, \mathrm{CDCl}_{3}\right) \delta 7.97(\mathrm{~s}, 2 \mathrm{H}), 7.82(\mathrm{~d}, \mathrm{~J}=8.21 \mathrm{~Hz}, 2 \mathrm{H})$, $7.65(\mathrm{~d}, \mathrm{~J}=7.39 \mathrm{~Hz}, 4 \mathrm{H}), 7.60-7.45(\mathrm{~m}, 9 \mathrm{H}), 7.37$ (d, J = 8.01 Hz, 2H), 6.63 (d, J = 8.00 Hz, 2H), $5.85(\mathrm{~s}, 2 \mathrm{H})$. The ${ }^{1} \mathrm{H}$ NMR data are consistent with a previous literature report. ${ }^{14}$ 


\section{SXIII. Optimization of Cross-Electrophile Coupling of Aryl Halides and Katritzky Salts}

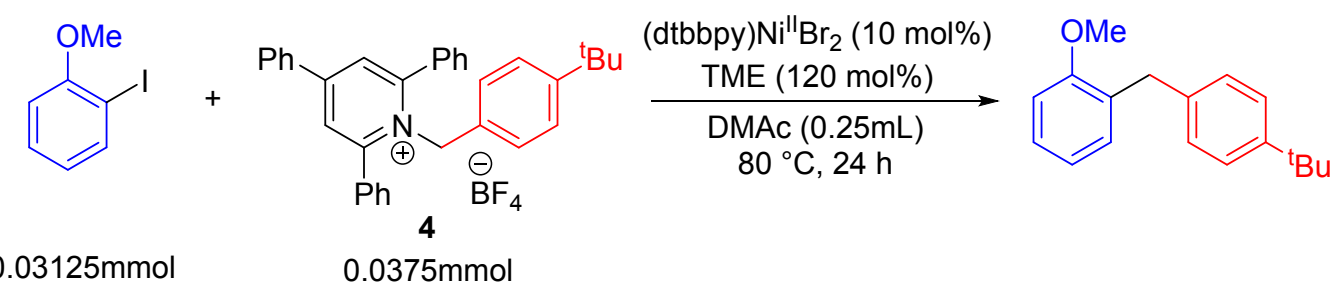

Figure S9. Cross-electrophile coupling of 2-iodoanisole and 4.

Representative Procedure:

In a glovebox under an $\mathrm{N}_{2}$ atmosphere, $4(20.3 \mathrm{mg}, 0.0375 \mathrm{mmol})$ and TME $(13.8 \mathrm{mg}$, $0.0375 \mathrm{mmol}$ ) were added to a 1 dram vial charged with a magnetic stir bar. A separate 2 dram vial was charged with (dtbbpy) $\mathrm{Ni}^{\mathrm{II}} \mathrm{Br}_{2}(6.0 \mathrm{mg}, 0.0125 \mathrm{mmol})$, DMAc $(1 \mathrm{~mL})$, and 2-iodoanisole $(16 \mu \mathrm{L}, 0.125 \mathrm{mmol})$ to create a stock solution in a glovebox under an $\mathrm{N}_{2}$ atmosphere. $0.26 \mathrm{~mL}$ of the stock solution was added to the vial containing $\mathbf{4}$ and TME to initiate catalysis. The reaction was capped tightly with a PTFE seal cap and stirred at $80^{\circ} \mathrm{C}$ for 24 hours. The reaction was opened to air and passed through a short plug of silica, which was washed with $5 \mathrm{~mL}$ EtOAc. The volatiles were removed, and the crude reaction mixture was dissolved in a solution of $\mathrm{CDCl}_{3}$ containing a known amount of hexamethylbenzene. Product yields were determined by integration of select peaks in the ${ }^{1} \mathrm{H}$ NMR spectrum against the hexamethylbenzene external standard.

Table S3. Yields of optimization studies for cross-electrophile coupling of 2-iodoanisole and 4.

\begin{tabular}{ccc}
\hline Entry & Deviation from standard conditions & Yield (\%) \\
\hline 1 & None & $\mathbf{9 0}$ \\
2 & 5 mol\% catalyst loading & 43 \\
3 & 2.5 mol\% catalyst loading & 29 \\
4 & DMI & 73 \\
5 & DMF & 5 \\
6 & $1,4-$ Dioxane & 0 \\
7 & No Ni catalyst & 0 \\
8 & No reductant & 0 \\
\hline
\end{tabular}


SXIV. Reductant Screen for Cross-Electrophile Coupling of 2-Iodoanisole and Katritzky

Salts 1, 3, and 4

Procedure:

See Section SXIII for representative experimental procedure. Note: The product yields for the coupling between 2-iodoanisole and 2 were also determined by ${ }^{1} \mathrm{H}$ NMR spectroscopy. However, only the product could be reliably traced due to the complexity of the spectra, and thus a detailed product profile was not available. See Table 3 in the manuscript for the product yields of the reaction between 2-iodoanisole and 2 .

Table S4. Cross-electrophile coupling of 2-iodoanisole and 1.

\begin{tabular}{|c|c|c|c|c|c|c|}
\hline \multicolumn{2}{|c|}{$0.03125 \mathrm{mmol}$} & ${ }_{1} \stackrel{\stackrel{\ominus}{\mathrm{BF}_{4}} \mathrm{Ar}}{ }$ & 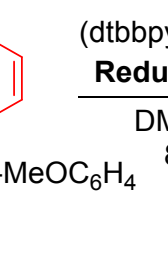 & $\begin{array}{l}\mathrm{Vill}^{\mathrm{ll}} \mathrm{Br}_{2}(10 \mathrm{~mol} \%) \\
\text { ant }(120 \mathrm{~mol} \%)\end{array}$ & $\mathrm{OMe}$ & 1 \\
\hline Reductant & $\begin{array}{c}\mathbf{E}^{\circ}(\mathbf{V} \mathbf{~ v s} \\
\left.\mathbf{F c} / \mathrm{Fc}^{+}\right)\end{array}$ & $\begin{array}{c}\text { Product } \\
\text { Yield } \\
(\%)\end{array}$ & ArI (\%) & Biaryl (\%) & $1(\%)$ & Bialkyl (\%) \\
\hline TPyE & -1.32 & 77 & $<1$ & $<1$ & $<1$ & 6 \\
\hline TDAE & -1.11 & 10 & 11 & 30 & 70 & $<1$ \\
\hline TAzE & -1.09 & $<1$ & 5 & 15 & 80 & $<1$ \\
\hline TPiE & -1.06 & $<1$ & 8 & 13 & 79 & $<1$ \\
\hline TME & -0.85 & $<1$ & 38 & 10 & 110 & $<1$ \\
\hline
\end{tabular}


Table S5. Cross-electrophile coupling of 2-iodoanisole and 3.

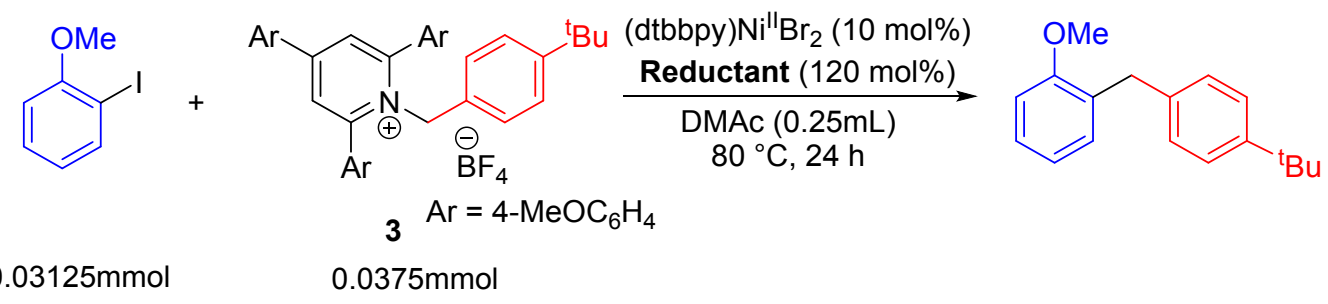

\begin{tabular}{ccccccc}
\hline Reductant & $\begin{array}{c}\mathbf{E}^{\circ}(\mathbf{V} \mathbf{v s} \\
\left.\mathbf{F c} / \mathbf{F c}^{+}\right)\end{array}$ & $\begin{array}{c}\text { Product } \\
\text { Yield } \\
\mathbf{( \% )}\end{array}$ & ArI (\%) & Biaryl (\%) & $\mathbf{3}(\%)$ & Bialkyl (\%) \\
\hline TPyE & -1.32 & 57 & $<1$ & 20 & 0 & 14 \\
TDAE & -1.11 & 99 & 0 & 0 & 0 & 16 \\
TAzE & -1.09 & 93 & 4 & 0 & 0 & 6 \\
TPiE & -1.06 & 99 & 0 & 0 & 0 & 3 \\
TME & -0.85 & 75 & 0 & 10 & 11 & 3 \\
TME & -0.85 & 93 & 0 & 2 & $<1$ & 0 \\
\hline
\end{tabular}

a $5 \mathrm{~mol}^{\%}$ (dtbbpy) $\mathrm{Ni}^{\mathrm{II}} \mathrm{Br}_{2}$ is used. 
Table S6. Cross-electrophile coupling of 2-iodoanisole and 4.

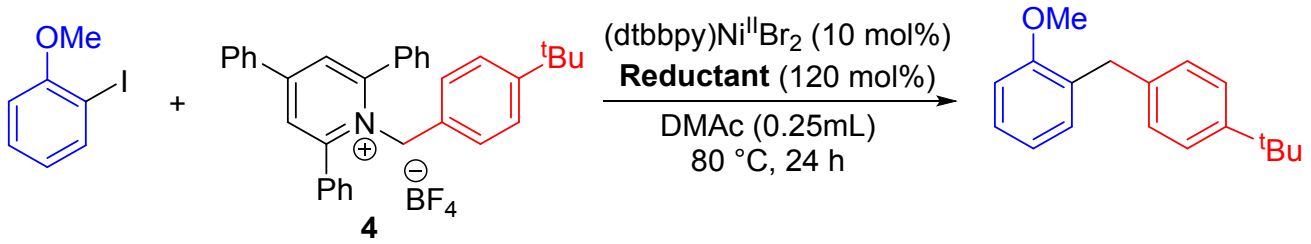

$0.03125 \mathrm{mmol}$

$0.0375 \mathrm{mmol}$

\begin{tabular}{ccccccc}
\hline Reductant & $\begin{array}{c}\mathbf{E}^{\circ}(\mathbf{V} \mathbf{v s} \\
\left.\mathbf{F c} / \mathbf{F c}^{+}\right)\end{array}$ & $\begin{array}{c}\text { Product } \\
\text { Yield } \\
\mathbf{( \% )}\end{array}$ & ArI (\%) & Biaryl (\%) & $\mathbf{4}(\%)$ & Bialkyl (\%) \\
\hline TPyE & -1.32 & 25 & 12 & 31 & 0 & 25 \\
TDAE & -1.11 & 29 & $<1$ & 36 & 0 & 29 \\
TAzE & -1.09 & 29 & 22 & 15 & 0 & 34 \\
TPiE & -1.06 & 30 & $<1$ & 22 & 0 & 27 \\
TME $^{\mathrm{a}}$ & -0.85 & 90 & 6 & $<1$ & 0 & 10 \\
\hline
\end{tabular}


SXV. ${ }^{1} \mathrm{H}$ NMR Yields of Products from Cross-Electrophile Coupling of Aryl Bromides and

\section{Katritzky Salt 4}

Procedure:

See section SXIII for representative experimental procedure. See Figure S10 below for individual reaction conditions.<smiles>COC(=O)c1ccc(Cc2ccc(C(=O)OC)cc2)cc1</smiles>

$0.03125 \mathrm{mmol}$

$0.0375 \mathrm{mmol}$

$28 \%$

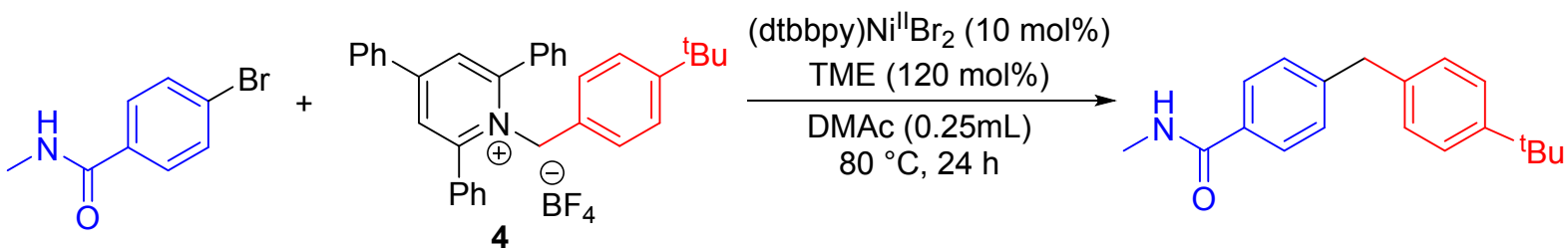

$0.03125 \mathrm{mmol}$

$0.0375 \mathrm{mmol}$

$4 \%$<smiles></smiles>

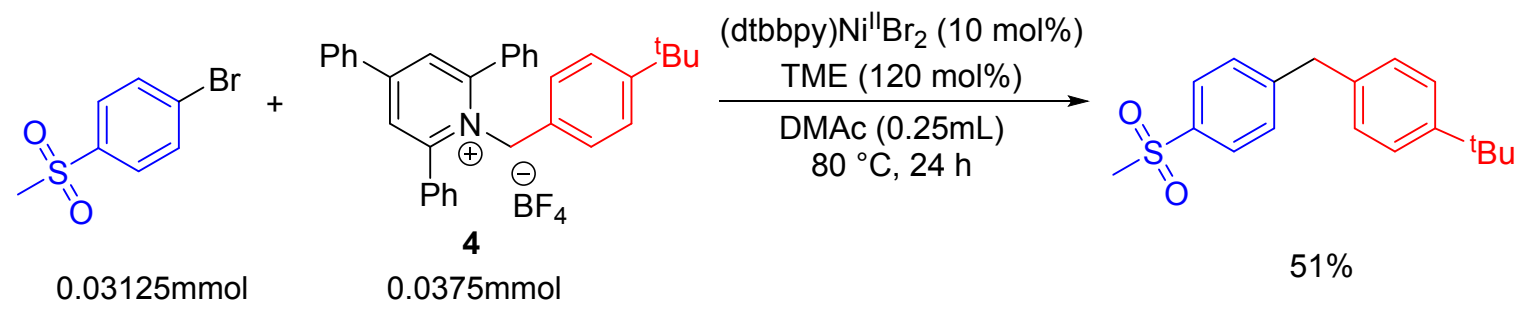

Figure S10. Cross-electrophile coupling of aryl bromides and 4. 
SXVI. ${ }^{1} \mathrm{H}$ NMR Yields of Products from Cross-Electrophile Coupling of Additional Aryl Iodide Substrates and Katritzky Salt 4

Procedure:

See section SXIII for representative experimental procedure. See Figure S11 below for individual reaction conditions.<smiles>Cc1ccccc1I</smiles><smiles></smiles>

$0.0375 \mathrm{mmol}$
(dtbbpy)Nill $\mathrm{Br}_{2}$ (10 mol\%)

TME (120 mol\%)

DMAc $(0.25 \mathrm{~mL})$ $80{ }^{\circ} \mathrm{C}, 24 \mathrm{~h}$<smiles>Cc1ccccc1Cc1ccc(C(C)(C)C)cc1</smiles>

$74 \%$<smiles>Ic1cccnc1</smiles>

$0.03125 \mathrm{mmol}$

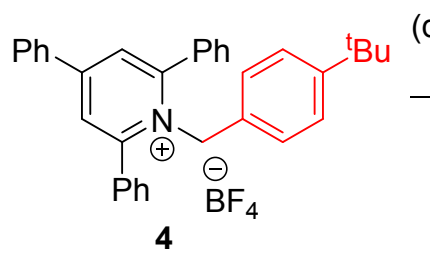
(dtbbpy) $\mathrm{Ni}^{\mathrm{ll}} \mathrm{Br}_{2}$ (10 mol\%)

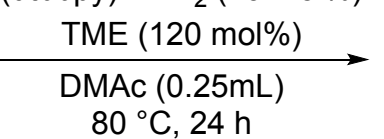<smiles>CC(C)(C)c1ccc(Cc2cccnc2)cc1</smiles>

$79 \%$<smiles>Clc1ccc(I)cc1</smiles><smiles></smiles>
(dtbbpy) $\mathrm{Ni}^{\mathrm{ll}} \mathrm{Br}_{2}$ (10 mol\%)

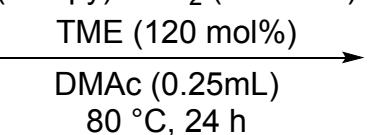<smiles>CC(C)(C)c1ccc(Cc2ccc(Cl)cc2)cc1</smiles>

$0.03125 \mathrm{mmol}$

$0.0375 \mathrm{mmol}$<smiles>Cc1cccc(C)c1I</smiles><smiles></smiles>

(dtbbpy) $\mathrm{Ni}^{\mathrm{ll}} \mathrm{Br}_{2}$ (10 mol\%)<smiles>COc1ccc(OC)cc1</smiles>
$80^{\circ} \mathrm{C}, 24 \mathrm{~h}$<smiles>Cc1cccc(C)c1Cc1ccc(C(C)(C)C)cc1</smiles>

$0 \%$

$0.03125 \mathrm{mmol}$

$0.0375 \mathrm{mmol}$<smiles>COc1cccc(OC)c1I</smiles><smiles>CC(C)(C(=O)OCc1ccc(Br)cc1)c1cc(-c2ccccc2)cc(-c2ccccc2)[n+]1-c1ccccc1</smiles>

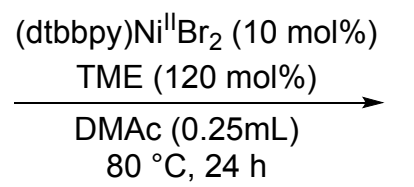<smiles>COc1cccc(OC)c1Cc1ccc(C(C)(C)C)cc1</smiles>
4

$0.03125 \mathrm{mmol}$

$0.0375 \mathrm{mmol}$

$0 \%$

Figure S11. Cross-electrophile coupling of additional aryl iodides and 4. 


\section{SXVII. Isolation Procedure and Characterization for Products of Cross-Electrophile}

\section{Coupling of Aryl Halides and Katritzky Salts}

Procedure for Isolation Scale Reactions of Following Substrates:

See section SXIII for experimental setup. Reactions were performed on a $0.125 \mathrm{mmol}$ scale of aryl electrophile (other reagents scaled linearly), and all the reagents were added directly to the reaction vial without introducing a stock soulution. See Figure 9 in the manuscript for individual reaction conditions. The reaction vial was removed from heat, allowed to cool to room temperature, and diluted with $0.5 \mathrm{~mL}$ EtOAc. The mixture was passed through a short silica plug ( 1.5 inches) in a glass pipette, which was rinsed with $8 \mathrm{~mL}$ EtOAc. The filtrate was concentrated to dryness, and the crude residue was purified by silica gel column chromatography.<smiles>CC(C)(C)c1ccc(Cc2ccc(C(C)(C)C)cc2)cc1</smiles><smiles>COc1ccc(Cc2ccc(Br)cc2)cc1</smiles><smiles>CC(C)(C)c1ccc(Cc2ccc(C(F)(F)F)cc2)cc1</smiles>

9a, bis-(4-(tert-butyl)phenyl)methane: Eluent: 1:9 toluene:hexanes. White solid, $74 \%$ yield $(24.9 \mathrm{mg})$. Isolated product contains $\sim 2 \%$ bialkyl which could not be separated. ${ }^{1} \mathrm{H}$ NMR (400 $\left.\mathrm{MHz}, \mathrm{CDCl}_{3}\right) \delta 7.31(\mathrm{~d}, J=8.1 \mathrm{~Hz}, 4 \mathrm{H}), 7.14(\mathrm{~d}, J=7.9 \mathrm{~Hz}, 4 \mathrm{H}), 3.93$ $(\mathrm{s}, 2 \mathrm{H}), 1.31(\mathrm{~s}, 18 \mathrm{H})$. The ${ }^{1} \mathrm{H}$ NMR data are consistent with a previous literature report. ${ }^{15}$

9b, 1-(tert-butyl)-4-(4-methoxybenzyl)benzene: Eluent: 1:4 toluene:hexanes. Colorless oil, $62 \%$ yield (15.4 mg). ${ }^{1} \mathrm{H}$ NMR (400 $\left.\mathrm{MHz}, \mathrm{CDCl}_{3}\right) \delta 7.30(\mathrm{~d}, J=8.3 \mathrm{~Hz}, 2 \mathrm{H}), 7.13-7.10(\mathrm{~m}, 4 \mathrm{H}), 6.83(\mathrm{~d}$, $J=8.6 \mathrm{~Hz}, 2 \mathrm{H}), 3.90$ (s, 2H), 3.78 (s, 3H), 1.30 (s, 9H). The ${ }^{1} \mathrm{H}$ NMR data are consistent with a previous literature report. ${ }^{16}$

9c, 1-(tert-butyl)-4-(4-trifluoromethyl)benzyl)benzene: Eluent: $10 \%$ toluene in hexanes. Colorless oil, $88 \%$ yield $(30.8 \mathrm{mg}) .{ }^{1} \mathrm{H}$ NMR $\left(400 \mathrm{MHz}, \mathrm{CDCl}_{3}\right) \delta 7.54(\mathrm{~d}, J=8.0 \mathrm{~Hz}, 2 \mathrm{H}), 7.34-7.30(\mathrm{~m}$, $4 \mathrm{H}), 7.11(\mathrm{~d}, J=8.5 \mathrm{~Hz}, 2 \mathrm{H}), 4.00(\mathrm{~s}, 2 \mathrm{H}), 1.33(\mathrm{~s}, 9 \mathrm{H}) .{ }^{13} \mathrm{C}\left\{{ }^{1} \mathrm{H}\right\}$ $\operatorname{NMR}\left(150 \mathrm{MHz}, \mathrm{CDCl}_{3}\right) \delta 149.46,145.54,137.08,129.35,128.67$, 128.53 (quartet, $\mathrm{J}=31.8 \mathrm{~Hz}$ ), $125.69,125.50$ (quartet, $\mathrm{J}=3.8 \mathrm{~Hz}$ ), 124.77 (quartet, $\mathrm{J}=271.7 \mathrm{~Hz}$ ), 41.36, 34.55, 31.50. ${ }^{19} \mathrm{~F}\left\{{ }^{1} \mathrm{H}\right\} \mathrm{NMR}$ $\left(376 \mathrm{MHz}, \mathrm{CDCl}_{3}\right) \delta-62.35$. (LRMS) GCMS EI $(\mathrm{m} / \mathrm{z})[\mathrm{M}]^{+}$ calculated for $\left[\mathrm{C}_{18} \mathrm{H}_{19} \mathrm{~F}_{3}\right]^{+} 2$ 292.1; found 292.2. 

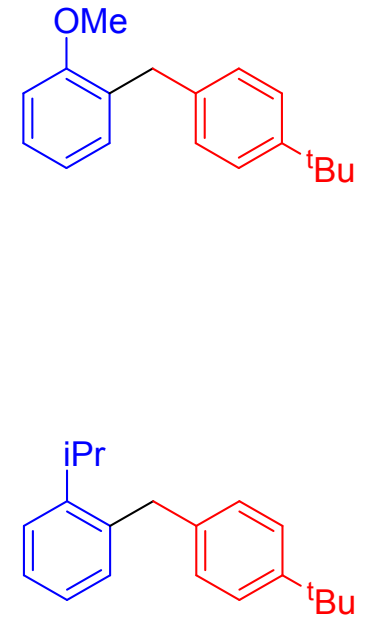

9d, 1-(4-tert-butyl)benzyl)-2-methoxybenzene: Eluent: 1\% EtOAc in 1:9 toluene:hexanes. White solid, 89\% yield $(22.1 \mathrm{mg})$. ${ }^{1} \mathrm{H}$ NMR $\left(400 \mathrm{MHz}, \mathrm{CDCl}_{3}\right) \delta 7.29(\mathrm{~d}, J=7.9 \mathrm{~Hz}, 2 \mathrm{H}), 7.19(\mathrm{t}, J=7.8$ $\mathrm{Hz}, 1 \mathrm{H}), 7.15$ (d, $J=7.9 \mathrm{~Hz}, 2 \mathrm{H}), 7.08$ (d, $J=7.5 \mathrm{~Hz}, 1 \mathrm{H}), 6.89-6.86$ (m, 2H), 3.95 (s, 2H), $3.83(\mathrm{~s}, 3 \mathrm{H}), 1.30(\mathrm{~s}, 9 \mathrm{H})$. The ${ }^{1} \mathrm{H}$ NMR data are consistent with a previous literature report. ${ }^{17}$

9e, 1-(4-(tert-butyl)benzyl)-2-isopropylbenzene: Eluent: 1:5 toluene:hexanes (first column) then $0-5 \%$ toluene in hexanes (second column). Colorless oil, 65\% yield (17.1 mg). ${ }^{1} \mathrm{H}$ NMR (400 $\left.\mathrm{MHz}, \mathrm{CDCl}_{3}\right) \delta$ 7.32-7.28 (m, 3H), 7.26-7.23 (m, 1H), 7.16-7.11 (m, 2H), 7.05 (d, $J=8.5 \mathrm{~Hz}, 2 \mathrm{H}), 4.04$ (s, 2H), 3.17 (septet, $J=6.9 \mathrm{~Hz}$, $1 \mathrm{H}), 1.31(\mathrm{~s}, 9 \mathrm{H}), 1.16(\mathrm{~d}, J=6.9 \mathrm{~Hz}, 6 \mathrm{H}) .{ }^{13} \mathrm{C}\left\{{ }^{1} \mathrm{H}\right\} \mathrm{NMR}(150 \mathrm{MHz}$, $\left.\mathrm{CDCl}_{3}\right) \delta 148.78,147.27,138.23,137.58,130.62,128.36,126.90$, $125.80,125.54,125.35,38.28,34.48,31.54,29.06,23.90$. (LRMS) GCMS EI $(\mathrm{m} / z)[\mathrm{M}]^{+}$calculated for $\left[\mathrm{C}_{20} \mathrm{H}_{26}\right]^{+} 266.2 ; 266.3$.

\begin{abstract}
9f, methyl 4-(4-(tert-butyl)benzyl)benzoate: Eluent: 100\% toluene. Colorless oil, 94\% yield (26.6 mg). ${ }^{1} \mathrm{H}$ NMR (400 $\left.\mathrm{MHz}, \mathrm{CDCl}_{3}\right) \delta 7.96(\mathrm{~d}, J=8.2 \mathrm{~Hz}, 2 \mathrm{H}), 7.32(\mathrm{~d}, J=8.3 \mathrm{~Hz}$, 2H), 7.27 (d, $J=8.0 \mathrm{~Hz}, 2 \mathrm{H}) 7.10(\mathrm{~d}, J=8.2 \mathrm{~Hz}, 2 \mathrm{H}), 4.00$ (s, 2H), 3.90 (s, 3H), $1.30(\mathrm{~s}, 9 \mathrm{H}) .{ }^{13} \mathrm{C}\left\{{ }^{1} \mathrm{H}\right\}$ NMR $(150 \mathrm{MHz}$, $\left.\mathrm{CDCl}_{3}\right) \delta 167.09,149.18,146.70,137.06,129.78,128.96$, 128.51, 127.97, 125.48, 52.00, 41.39, 34.38, 31.35. (LRMS) GCMS EI $(\mathrm{m} / z)[\mathrm{M}]^{+}$calculated for $\left[\mathrm{C}_{19} \mathrm{H}_{22} \mathrm{O}_{2}\right]^{+} 282.2$; found 282.2.
\end{abstract}

9g, 4-(4-(tert-butyl)benzyl)phenol: Eluent: 25\% EtOAc in hexanes (first column) then 100\% DCM (second column). White solid, 60\% yield $(17.3 \mathrm{mg}) .{ }^{1} \mathrm{H}$ NMR $\left(400 \mathrm{MHz}, \mathrm{CDCl}_{3}\right) \delta 7.31(\mathrm{~d}, J=8.3 \mathrm{~Hz}$, 2H), 7.12-7.06 (m, 4H), 6.76 (d, J=8.6 Hz, 2H), 4.60 (br s, 1H), 3.89 $(\mathrm{s}, 2 \mathrm{H}), 1.31(\mathrm{~s}, 9 \mathrm{H})$. The ${ }^{1} \mathrm{H}$ NMR data are consistent with a previous literature report. ${ }^{18}$ 

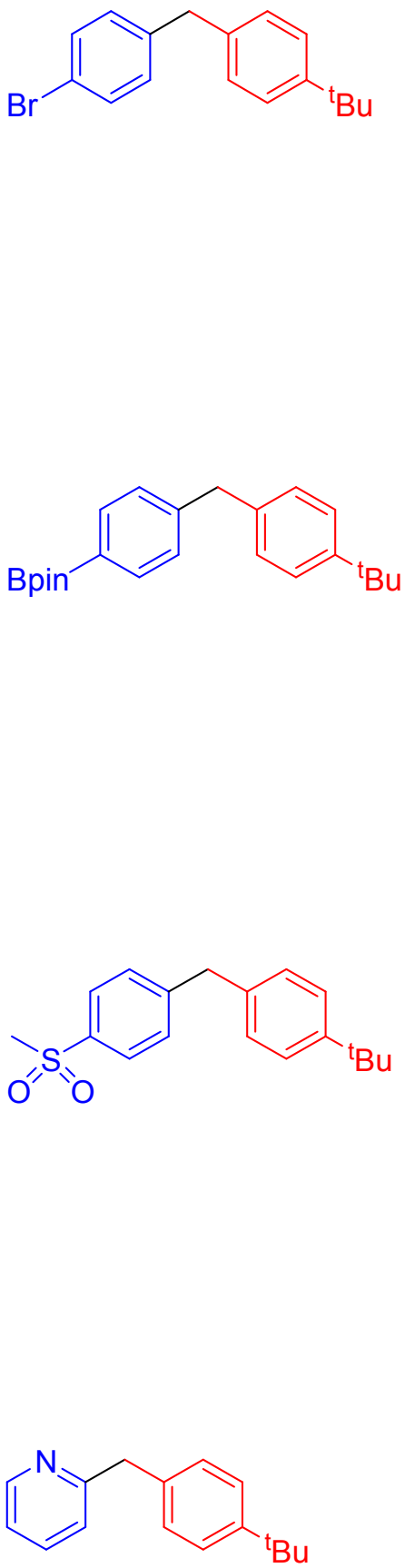

9h, 1-bromo-4-(4-(tert-butyl)benzyl)benzene: Eluent: 15:85 toluene:hexanes. White solid, $81 \%$ yield $(30.7 \mathrm{mg})$. Isolated product contains $\sim 2 \%$ bialkyl which could not be separated. ${ }^{1} \mathrm{H}$ NMR (400 $\left.\mathrm{MHz}, \mathrm{CDCl}_{3}\right) \delta 7.41(\mathrm{~d}, J=8.3 \mathrm{~Hz}, 2 \mathrm{H}), 7.32$ (d, $\left.J=8.2 \mathrm{~Hz}, 2 \mathrm{H}\right)$, 7.11-7.07 (m, 4H), $3.91(\mathrm{~s}, 2 \mathrm{H}), 1.31(\mathrm{~s}, 9 \mathrm{H}) .{ }^{13} \mathrm{C}\left\{{ }^{1} \mathrm{H}\right\}$ NMR $(150$ $\left.\mathrm{MHz}, \mathrm{CDCl}_{3}\right) \delta 149.25,140.41,137.54,131.61,130.83,128.57$, 125.60, 119.98, 40.92, 37.54, 34.52, 31.51. (LRMS) GCMS EI $(m / z)$ $[\mathrm{M}]^{+}$calculated for $\left[\mathrm{C}_{17} \mathrm{H}_{19} \mathrm{Br}\right]^{+} 302.1 ; 302.1$.

9i, 2-(4-(4-(tert-butyl)benzyl)phenyl)-4,4,5,5-tetramethyl-1,3,2dioxaborolane: Eluent: $100 \%$ toluene. White solid, $72 \%$ yield (31.4 mg). ${ }^{1} \mathrm{H}$ NMR (400 MHz, $\left.\mathrm{CDCl}^{3}\right) \delta 7.74(\mathrm{~d}, J=7.9 \mathrm{~Hz}, 2 \mathrm{H}), 7.29$ $(\mathrm{d}, J=8.3 \mathrm{~Hz}, 2 \mathrm{H}), 7.22(\mathrm{~d}, J=8.0 \mathrm{~Hz}, 2 \mathrm{H}), 7.10(\mathrm{~d}, J=8.3 \mathrm{~Hz}$, 2H), 3.96 (s, 2H), 1.33 (s, 12H), $1.29(\mathrm{~s}, 9 \mathrm{H}) .{ }^{13} \mathrm{C}\left\{{ }^{1} \mathrm{H}\right\}$ NMR $(150$ $\left.\mathrm{MHz}, \mathrm{CDCl}_{3}\right) \delta 148.83,144.57,137.83,134.97,128.44,125.32$, 83.63, 41.62, 34.33, 31.36, 30.92, 24.82. (LRMS) GCMS EI $(m / z)$ $[\mathrm{M}]^{+}$calculated for $\left[\mathrm{C}_{23} \mathrm{H}_{31} \mathrm{BO}_{2}\right]^{+} 350.2$; found 350.3 .

9j, 1-(tert-butyl)-4-(4-methylsulfonyl)benzyl)benzene: Eluent: gradient of 20-30\% EtOAc in hexanes. White solid, 89\% yield (32.3 mg). ${ }^{1} \mathrm{H}$ NMR $\left(400 \mathrm{MHz}, \mathrm{CDCl}_{3}\right) \delta 7.85(\mathrm{~d}, \mathrm{~J}=6.7 \mathrm{~Hz}, 2 \mathrm{H}), 7.39(\mathrm{~d}$, $\mathrm{J}=8.2 \mathrm{~Hz}, 2 \mathrm{H}), 7.31(\mathrm{~d}, \mathrm{~J}=6.8 \mathrm{~Hz}, 2 \mathrm{H}), 7.10(\mathrm{~d}, \mathrm{~J}=8.2 \mathrm{~Hz}, 2 \mathrm{H}), 4.03$ (s, 2H), $3.03(\mathrm{~s}, 3 \mathrm{H}), 1.31(\mathrm{~s}, 9 \mathrm{H}) .{ }^{13} \mathrm{C}\left\{{ }^{1} \mathrm{H}\right\} \mathrm{NMR}\left(150 \mathrm{MHz}, \mathrm{CDCl}_{3}\right)$ $\delta 149.69,148.05,138.36,136.50,129.96,128.70,127.73,125.79$, 44.73, 41.44, 34.57, 31.48. (LRMS) GCMS EI $(\mathrm{m} / \mathrm{z})[\mathrm{M}]^{+}$calculated for $\left[\mathrm{C}_{18} \mathrm{H}_{22} \mathrm{O}_{2} \mathrm{~S}\right]^{+} 302.1 ; 302.2$.

9k, 2-(4-tert-butyl)benzyl)pyridine: Eluent: gradient of 10-20\% EtOAc in hexanes. Pale yellow oil, $71 \%$ yield $(19.2 \mathrm{mg}) .{ }^{1} \mathrm{H}$ NMR $\left(400 \mathrm{MHz}, \mathrm{CDCl}_{3}\right) \delta 8.56(\mathrm{~d}, J=4.8 \mathrm{~Hz}, 1 \mathrm{H}), 7.59(\mathrm{t}, J=7.7 \mathrm{~Hz}, 1 \mathrm{H})$, 7.33 (d, $J=7.9 \mathrm{~Hz}, 2 \mathrm{H}), 7.20$ (d, $J=7.9 \mathrm{~Hz}, 2 \mathrm{H}), 7.14-7.10(\mathrm{~m}, 2 \mathrm{H})$, $4.14(\mathrm{~s}, 2 \mathrm{H}), 1.30(\mathrm{~s}, 9 \mathrm{H}) .{ }^{13} \mathrm{C}\left\{{ }^{1} \mathrm{H}\right\} \mathrm{NMR}\left(150 \mathrm{MHz}, \mathrm{CDCl}_{3}\right) \delta$ $160.78,149.53,148.20,137.81,135.85,128.90,125.74,123.79$, 121.69, 43.55, 34.54, 31.48. (LRMS) GCMS EI $(m / z)[\mathrm{M}]^{+}$ calculated for $\left[\mathrm{C}_{16} \mathrm{H}_{19} \mathrm{~N}\right]^{+}$225.2; found 225.2. 

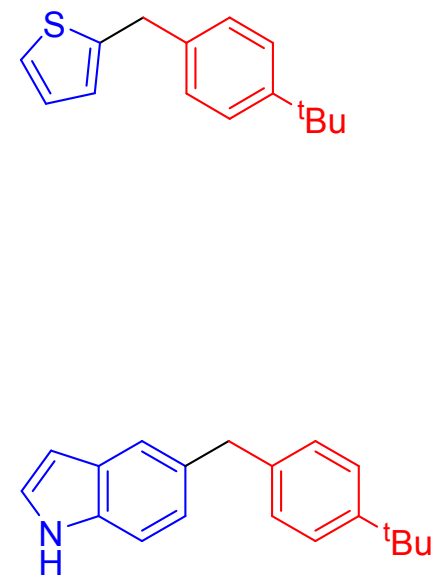

9l, 2-(4-tert-butyl)benzyl)thiophene: Eluent: gradient of 1-10\% toluene in hexanes. Colorless oil, 52\% yield $(14.4 \mathrm{mg}) .{ }^{1} \mathrm{H}$ NMR $\left(400 \mathrm{MHz}, \mathrm{CDCl}_{3}\right) \delta 7.33(\mathrm{~d}, J=6.7 \mathrm{~Hz}, 2 \mathrm{H}), 7.19(\mathrm{~d}, J=8.2 \mathrm{~Hz}, 2 \mathrm{H})$, $7.15(\mathrm{~d}, J=5.2 \mathrm{~Hz}, 1 \mathrm{H}), 6.94-6.92(\mathrm{~m}, 1 \mathrm{H}), 6.82(\mathrm{~m}, 1 \mathrm{H}), 4.13$ (s, $2 \mathrm{H}), 1.31(\mathrm{~s}, 9 \mathrm{H})$. The ${ }^{1} \mathrm{H}$ NMR data are consistent with a previous literature report. ${ }^{19}$

9m, 5-(4-(tert-butyl)benzyl)-1H-indole: Eluent: gradient of 10$20 \%$ EtOAc in hexanes. Pale yellow oil, $50 \%$ yield (15.8 mg). Note: The product slowly decomposes in air and should be stored under inert atmosphere in the dark. ${ }^{1} \mathrm{H} \mathrm{NMR}\left(400 \mathrm{MHz}^{\mathrm{CDCl}} \mathrm{CD}_{3}\right) \delta 8.06(\mathrm{br}$ $\mathrm{s}, 1 \mathrm{H}), 7.49(\mathrm{~s} 1 \mathrm{H}), 7.31(\mathrm{~m}, 3 \mathrm{H}), 7.19-7.17(\mathrm{~m}, 3 \mathrm{H}), 7.07$ (d, $J=6.6$ $\mathrm{Hz}, 1 \mathrm{H}), 6.50(\mathrm{~m}, 1 \mathrm{H}), 4.07(\mathrm{~s}, 2 \mathrm{H}), 1.31(\mathrm{~s}, 9 \mathrm{H}) .{ }^{13} \mathrm{C}\left\{{ }^{1} \mathrm{H}\right\}$ NMR $\left(150 \mathrm{MHz}, \mathrm{CDCl}_{3}\right) \delta 148.64,139.42,134.54,132.81,128.58$, $128.21,125.41,124.45,123.67,120.72,111.05,102.53,41.64$, 34.47, 31.55. (LRMS) GCMS EI $(\mathrm{m} / \mathrm{z})[\mathrm{M}]^{+}$calculated for $\left[\mathrm{C}_{19} \mathrm{H}_{21} \mathrm{~N}\right]^{+}$263.2; found 263.2.

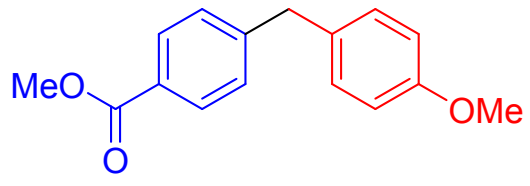

9n, methyl 4-(4-methoxybenzyl)benzoate: Eluent: gradient of 10-20\% EtOAc in hexanes. Colorless oil, 84\% yield $(27.6 \mathrm{mg}) .{ }^{1} \mathrm{H}$ NMR $\left(400 \mathrm{MHz}, \mathrm{CDCl}_{3}\right) \delta 7.95(\mathrm{~d}$, $J=8.3 \mathrm{~Hz}, 2 \mathrm{H}), 7.24(\mathrm{~d}, J=8.0 \mathrm{~Hz}, 2 \mathrm{H}), 7.09$ (d, $J=8.2 \mathrm{~Hz}$, $2 \mathrm{H}), 6.84$ (d, $J=8.6 \mathrm{~Hz}, 2 \mathrm{H}), 3.97$ (s, 2H), 3.89 (s, 3H), 3.79 $(\mathrm{s}, 3 \mathrm{H})$. The ${ }^{1} \mathrm{H}$ NMR data are consistent with a previous literature report. ${ }^{20}$

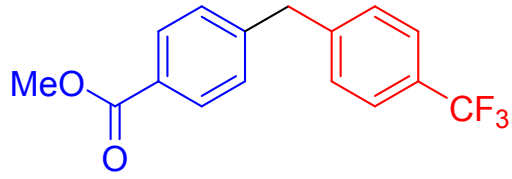

9o, methyl 4-(4-(trifluoromethyl)benzyl)benzoate: Eluent: 1:1 toluene:hexanes. Pale yellow oil, 82\% yield (31.2 mg). ${ }^{1} \mathrm{H}$ NMR (400 MHz, $\left.\mathrm{CDCl}_{3}\right) \delta 7.98$ (d, $J=8.3 \mathrm{~Hz}$, 2H), 7.55 (d, J=8.0 Hz, 2H), 7.29-7.23 (m, 4H), $4.08(\mathrm{~s}, 2 \mathrm{H})$, $3.90(\mathrm{~s}, 3 \mathrm{H})$. The ${ }^{1} \mathrm{H}$ NMR data are consistent with a previous literature report. ${ }^{16}$ 


\section{SXVIII. Procedure for 1 mmol Scale Reaction to Form Product 9f}

Procedure:

A $100 \mathrm{~mL}$ Schlenk flask was removed from an oven at $130{ }^{\circ} \mathrm{C}$ and was immediately transferred into a glovebox under an $\mathrm{N}_{2}$ atmosphere. The Schlenk flask was allowed to cool to room temperature and was charged with methyl 4-iodobenzoate (270 mg, $1 \mathrm{mmol}), 4$ (653 mg, 1.2 mmol), (dtbbpy) $\mathrm{Ni}^{\mathrm{II}} \mathrm{Br}_{2}(48 \mathrm{mg}, 0.1 \mathrm{mmol})$, TME (440 mg, $\left.1.2 \mathrm{mmol}\right), \mathrm{DMAc}(8 \mathrm{~mL})$ and a magnetic stir bar. The flask was sealed, quickly removed from the glovebox, and placed in an oil bath. The reaction was stirred at $80^{\circ} \mathrm{C}$ for 24 hours. After 24 hours, the reaction was allowed to cool to room temperature, and the reaction mixture was filtered through a short pad of celite. The flask and celite were rinsed with $15 \mathrm{~mL}$ of EtOAc. The filtrate was concentrated in vacuo, and the crude product was purified by silica gel column chromatography using $100 \%$ toluene as eluent. Product 9f was obtained in an $85 \%$ yield $(247 \mathrm{mg})$.

${ }^{1} \mathrm{H}$ NMR $\left(400 \mathrm{MHz}, \mathrm{CDCl}_{3}\right) \delta 7.96(\mathrm{~d}, J=8.3 \mathrm{~Hz}, 2 \mathrm{H}), 7.32(\mathrm{~d}, J=8.3 \mathrm{~Hz}, 2 \mathrm{H}), 7.27$ (d, $J=7.9 \mathrm{~Hz}$, 2H) $7.10(\mathrm{~d}, J=8.6 \mathrm{~Hz}, 2 \mathrm{H}), 4.00(\mathrm{~s}, 2 \mathrm{H}), 3.90(\mathrm{~s}, 3 \mathrm{H}), 1.30(\mathrm{~s}, 9 \mathrm{H})$. The ${ }^{1} \mathrm{H}$ NMR data are consistent with the results collected on a smaller scale (see Section SXVII, product 9f). 


\section{SXIX. NMR Spectra of Isolated Compounds}

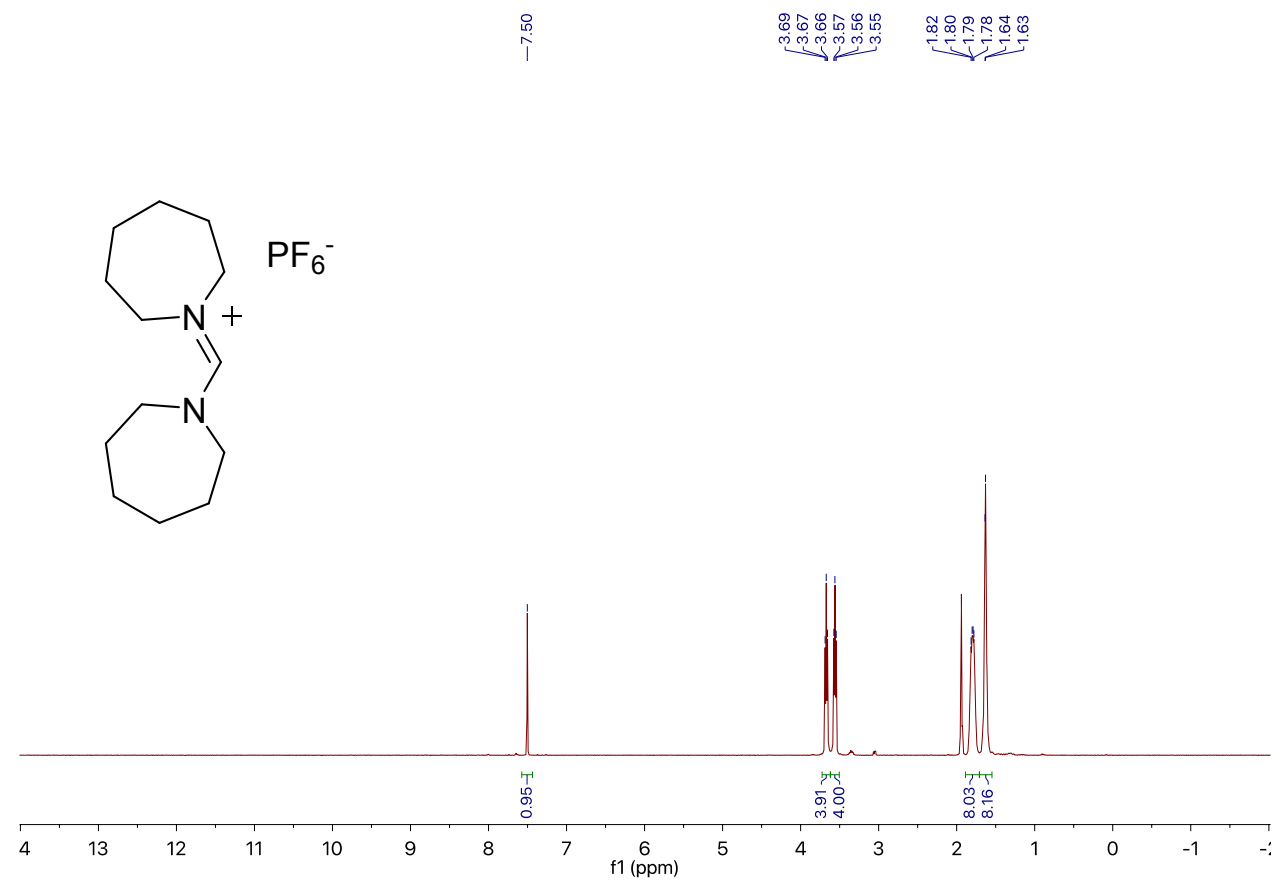

Figure S12. ${ }^{1} \mathrm{H}$ NMR spectrum $\left(400 \mathrm{MHz}, \mathrm{CD}_{3} \mathrm{CN}\right)$ of $2 \mathrm{c}-\mathrm{Az}$.

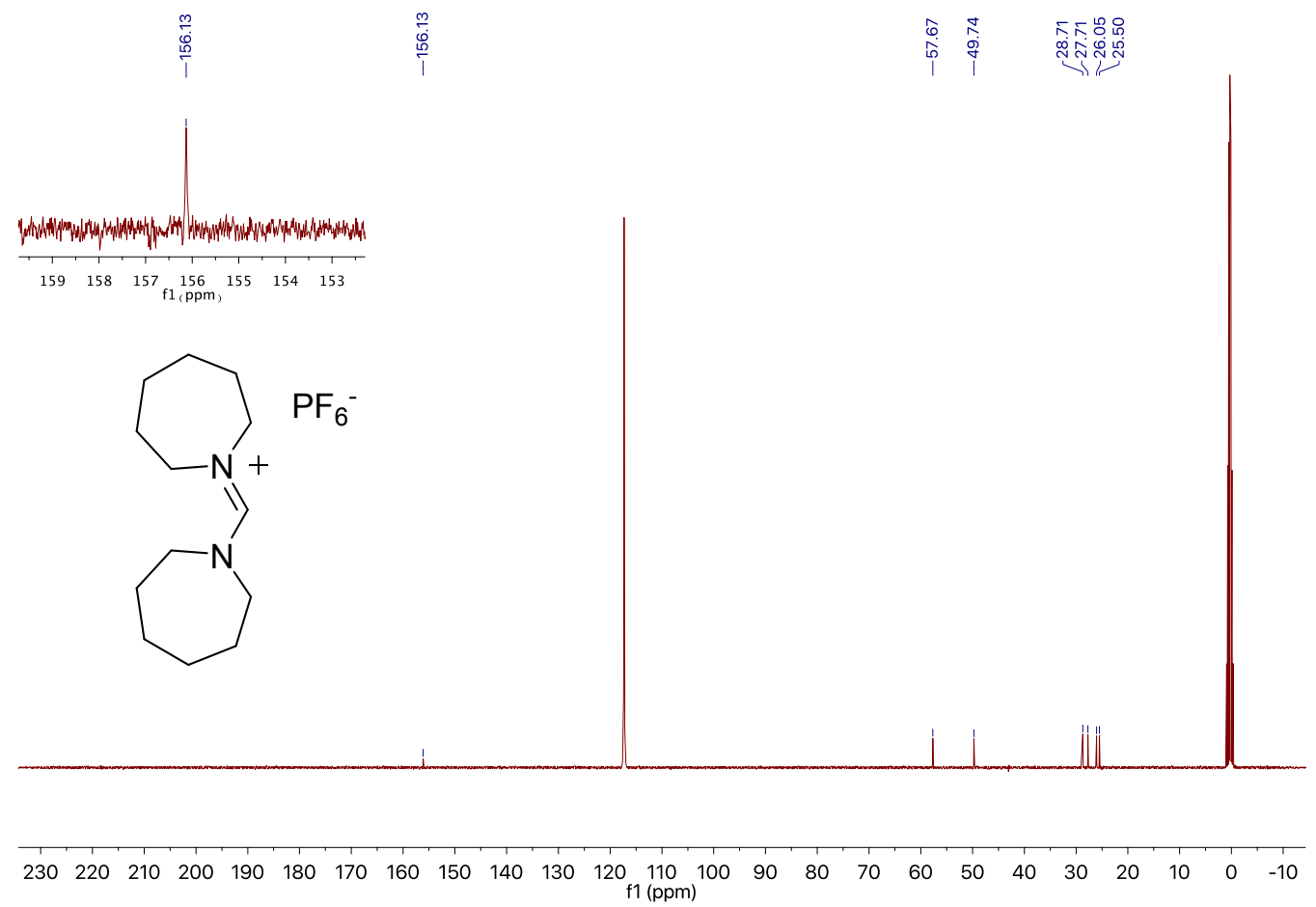

Figure S13. ${ }^{13} \mathrm{C}\left\{{ }^{1} \mathrm{H}\right\} \mathrm{NMR}$ spectrum $\left(100 \mathrm{MHz}, \mathrm{CD}_{3} \mathrm{CN}\right)$ of $\mathbf{2 c}-\mathbf{A z}$. 


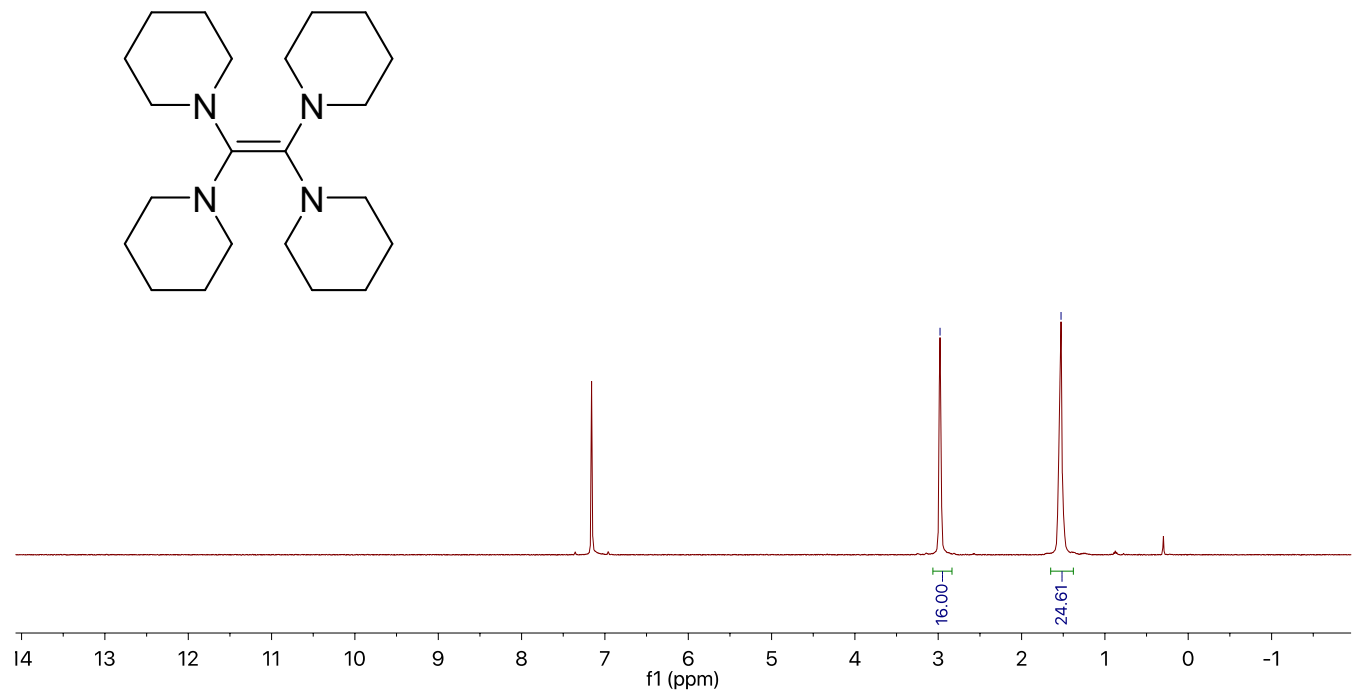

Figure S14. ${ }^{1} \mathrm{H}$ NMR spectrum $\left(400 \mathrm{MHz}, \mathrm{C}_{6} \mathrm{D}_{6}\right)$ of TPiE.
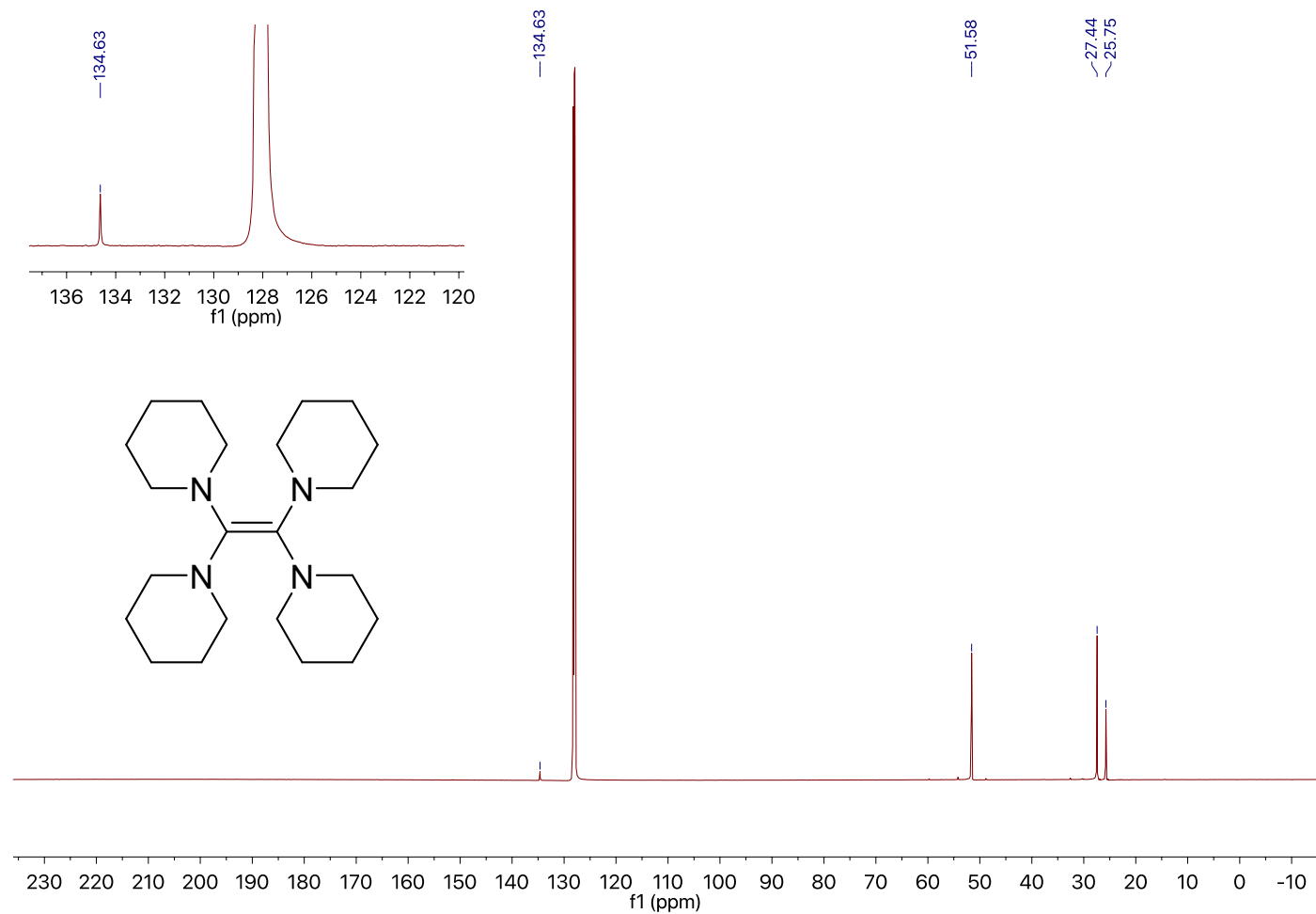

Figure S15. ${ }^{13} \mathrm{C}\left\{{ }^{1} \mathrm{H}\right\}$ NMR spectrum $\left(150 \mathrm{MHz}, \mathrm{C}_{6} \mathrm{D}_{6}\right)$ of TPiE. 


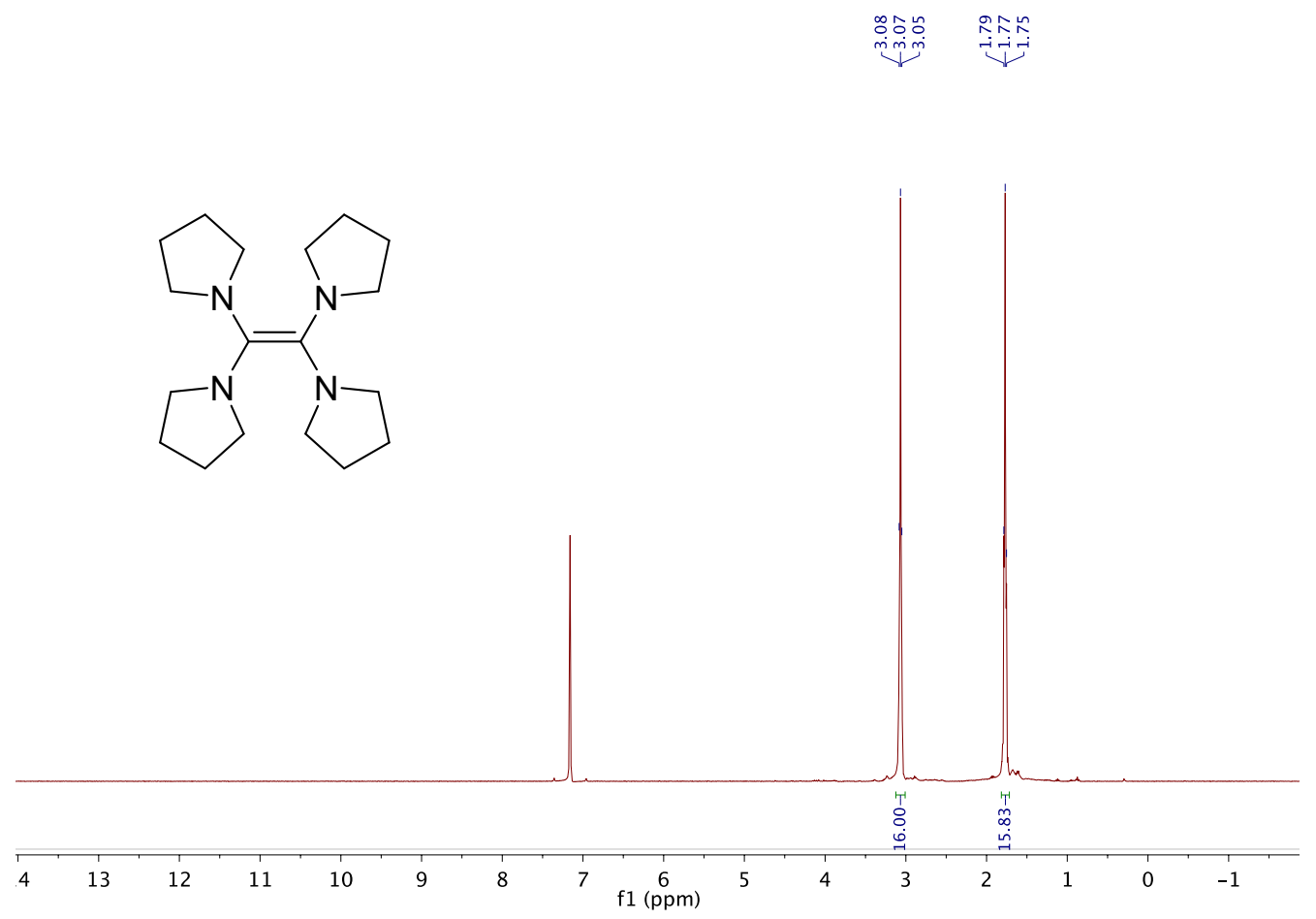

Figure S16. ${ }^{1} \mathrm{H}$ NMR spectrum $\left(400 \mathrm{MHz}, \mathrm{C}_{6} \mathrm{D}_{6}\right)$ of TPyE.

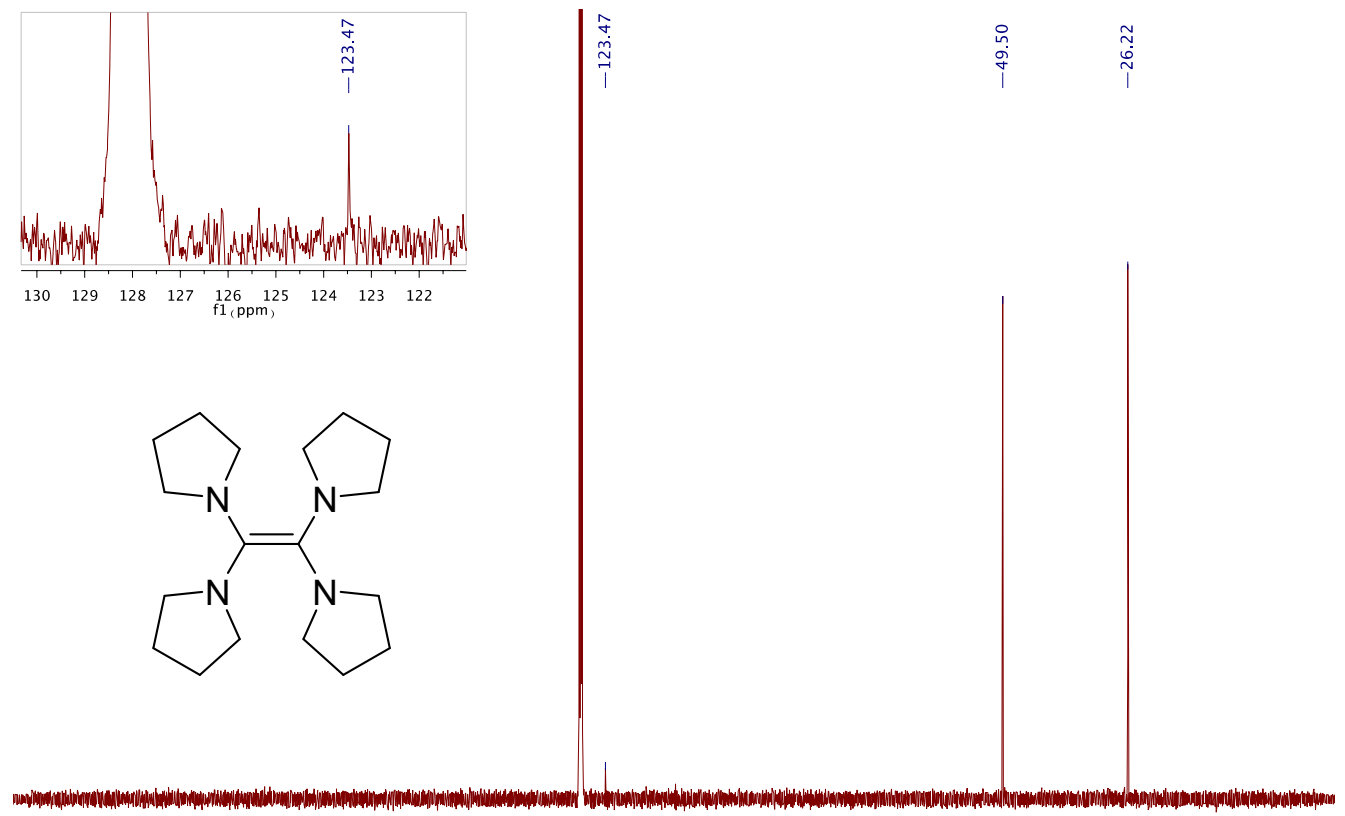

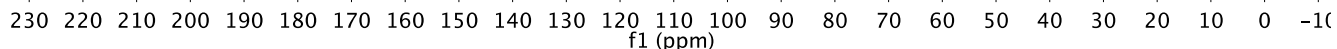

Figure S17. ${ }^{13} \mathrm{C}\left\{{ }^{1} \mathrm{H}\right\}$ NMR spectrum $\left(100 \mathrm{MHz}, \mathrm{C}_{6} \mathrm{D}_{6}\right)$ of TPyE. 

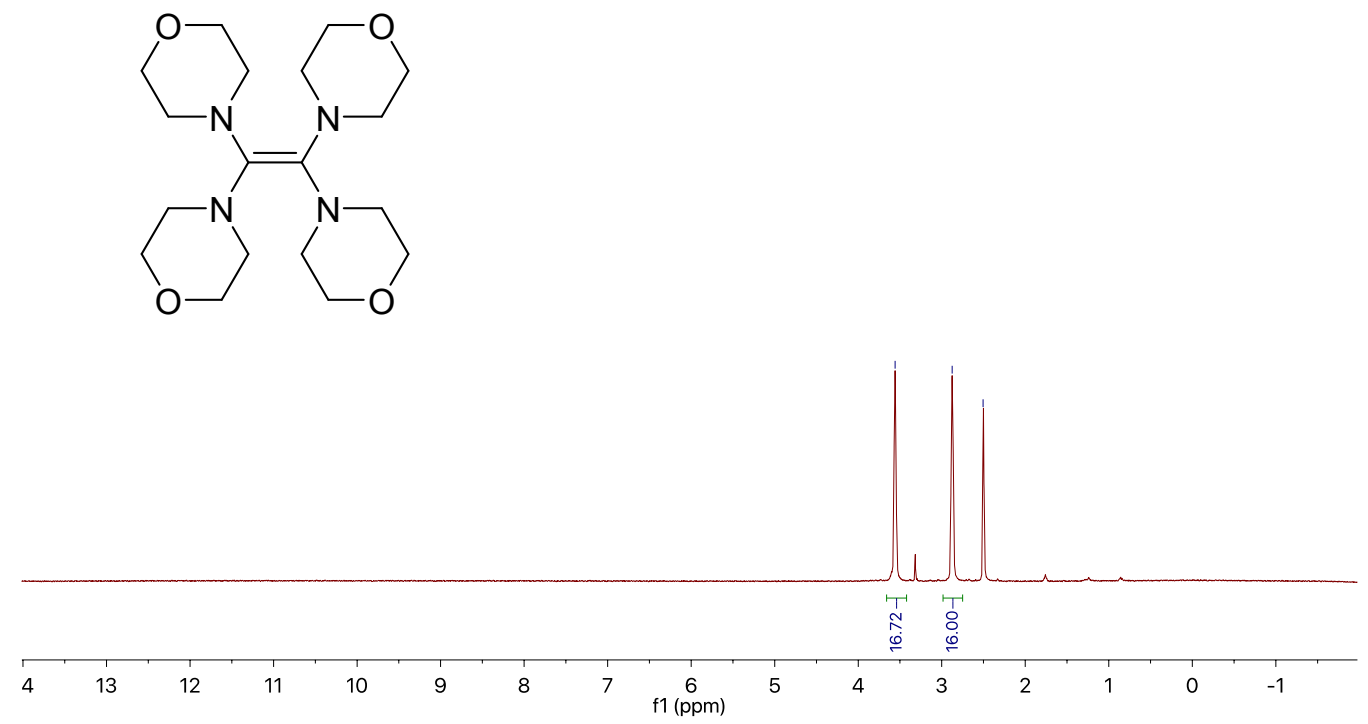

Figure S18. ${ }^{1} \mathrm{H}$ NMR spectrum $\left(400 \mathrm{MHz}, \mathrm{DMSO}-\mathrm{d}_{6}\right)$ of TME.
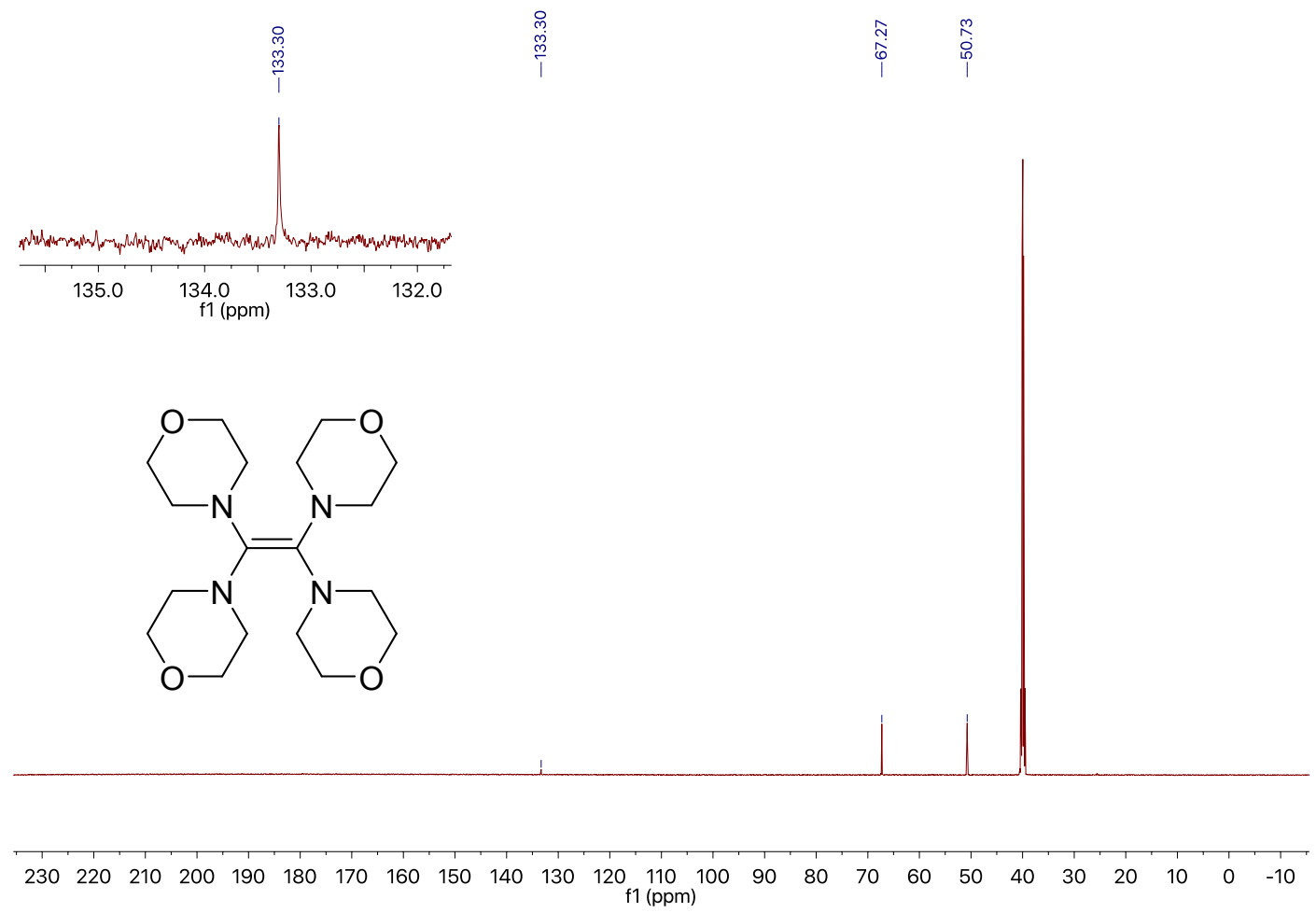

Figure S19. ${ }^{13} \mathrm{C}\left\{{ }^{1} \mathrm{H}\right\}$ NMR spectrum (150 MHz, DMSO-d $\left.\mathrm{d}_{6}\right)$ of TME. 


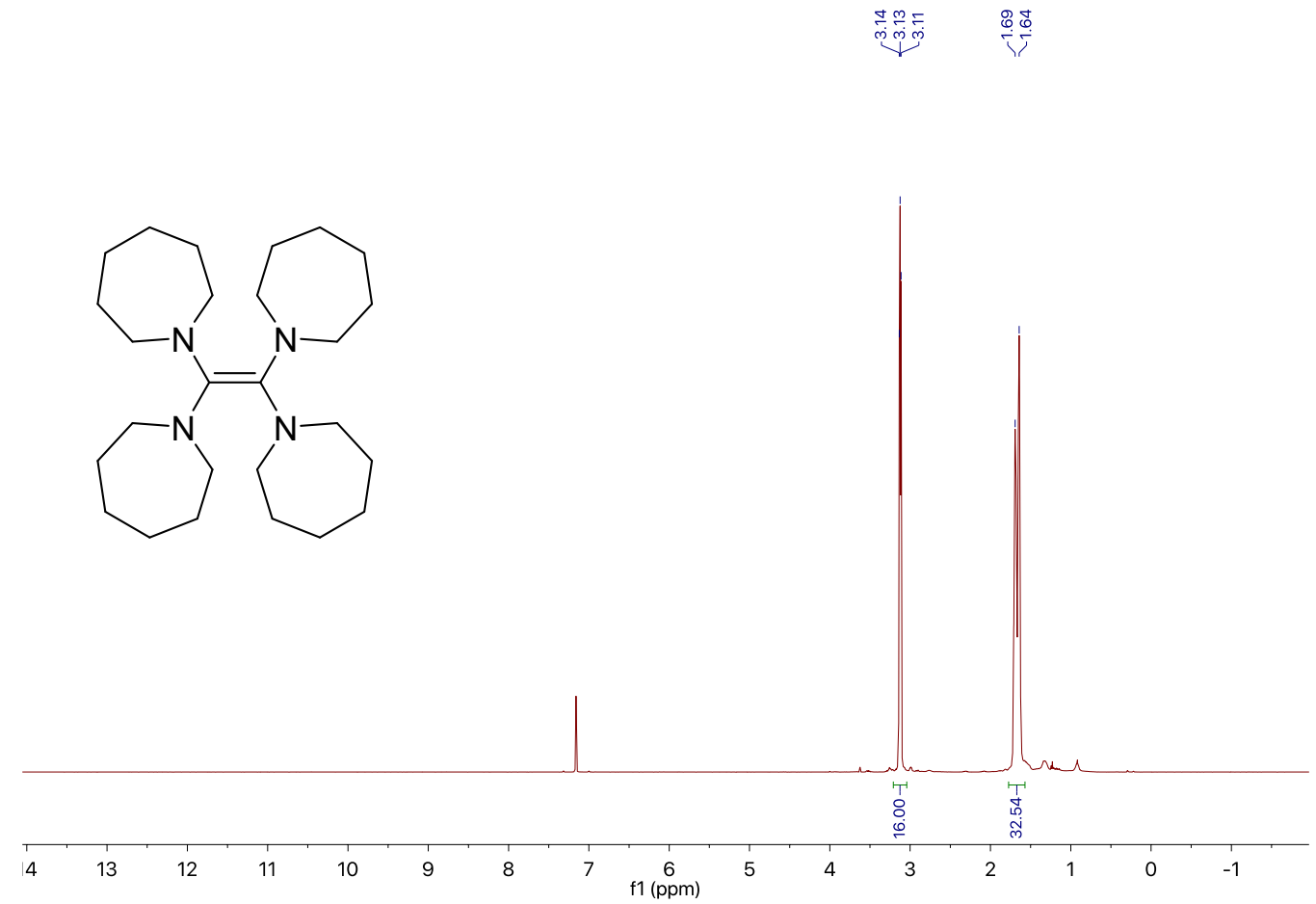

Figure S20. ${ }^{1} \mathrm{H}$ NMR spectrum $\left(500 \mathrm{MHz}, \mathrm{C}_{6} \mathrm{D}_{6}\right)$ of TAzE.

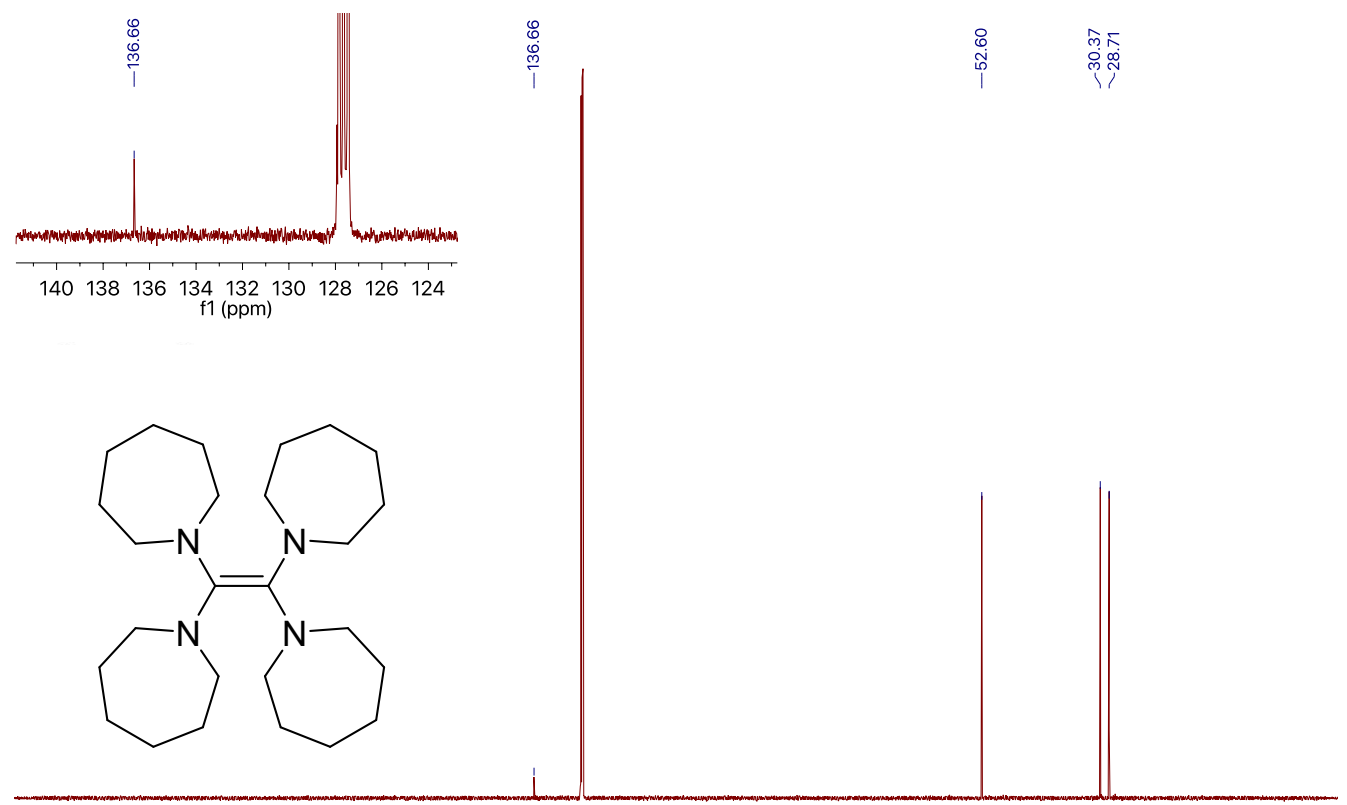

$\begin{array}{lllllllllllllllllllllllll}230 & 220 & 210 & 200 & 190 & 180 & 170 & 160 & 150 & 140 & 130 & 120 & 110 & 100 & 90 & 80 & 70 & 60 & 50 & 40 & 30 & 20 & 10 & 0 & -10\end{array}$

Figure S21. ${ }^{13} \mathrm{C}\left\{{ }^{1} \mathrm{H}\right\}$ NMR spectrum $\left(125 \mathrm{MHz}, \mathrm{C}_{6} \mathrm{D}_{6}\right)$ of TAzE. 

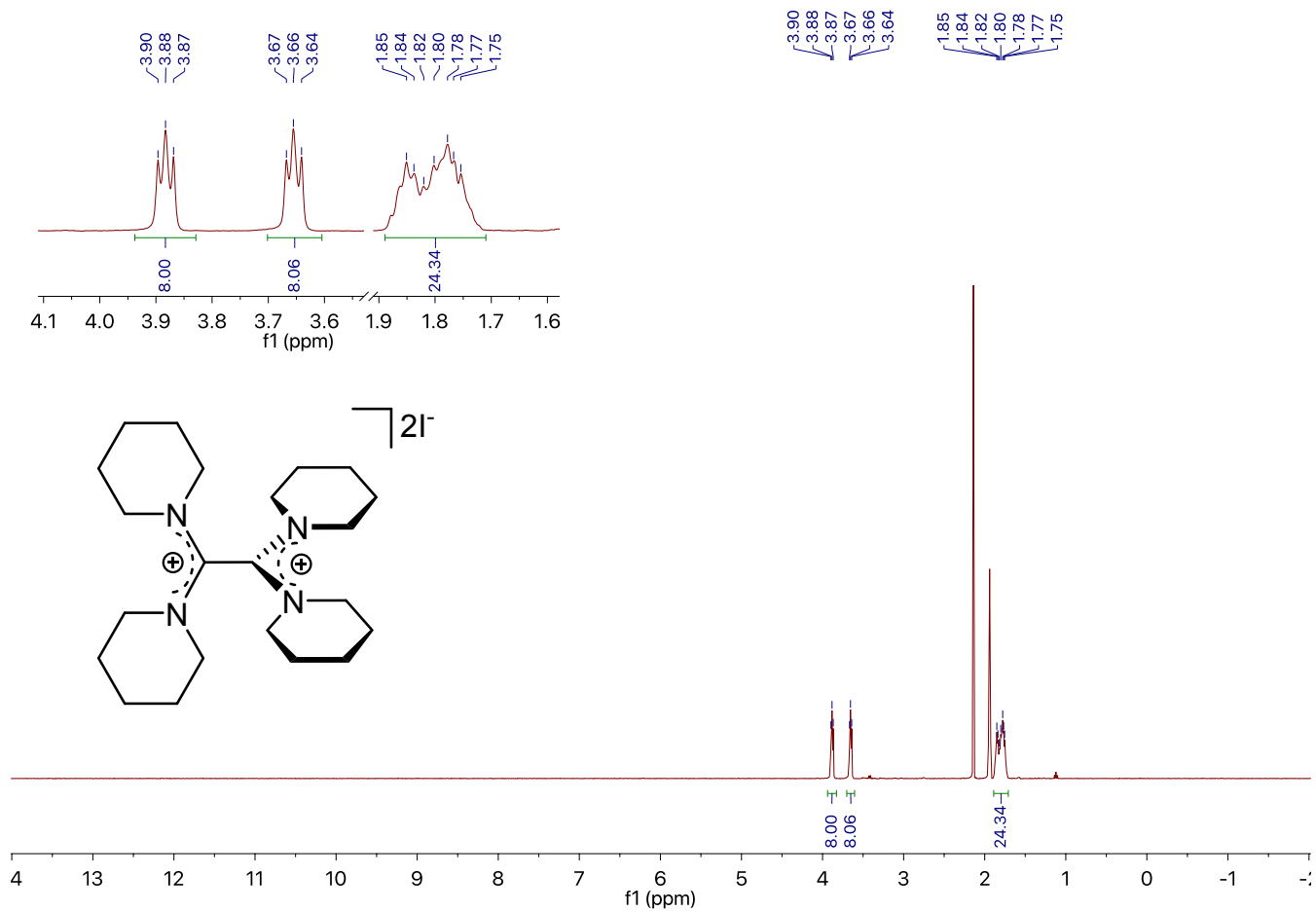

Figure S22. ${ }^{1} \mathrm{H}$ NMR spectrum $\left(400 \mathrm{MHz}, \mathrm{CD}_{3} \mathrm{CN}\right)$ of $[\mathrm{TPiE}]^{2+} 2[\mathrm{l}]$ -

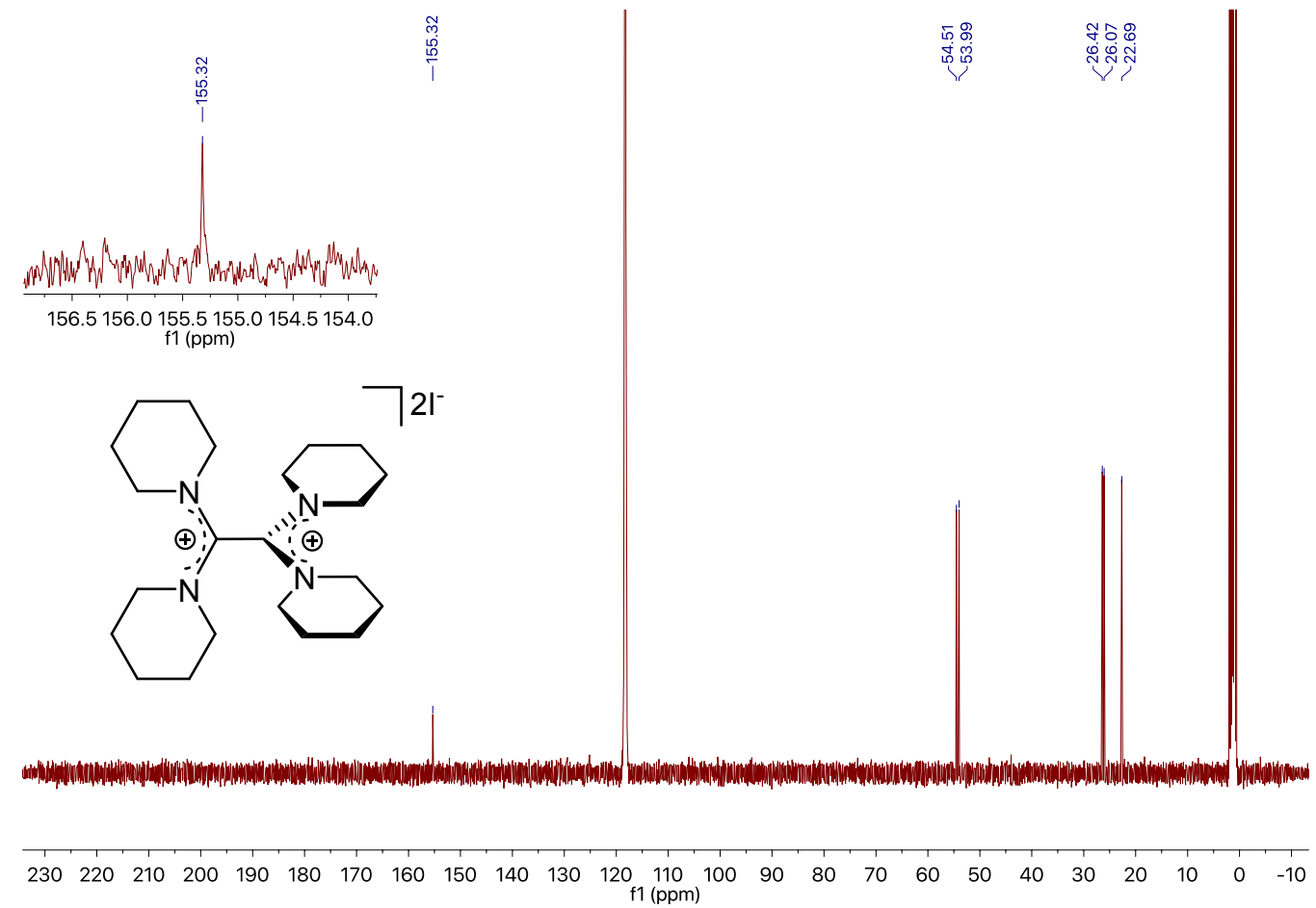

Figure S23. ${ }^{13} \mathrm{C}\left\{{ }^{1} \mathrm{H}\right\} \mathrm{NMR}$ spectrum $\left(100 \mathrm{MHz}, \mathrm{CD}_{3} \mathrm{CN}\right)$ of $[\mathrm{TPiE}]^{2+} 2[1]$. 


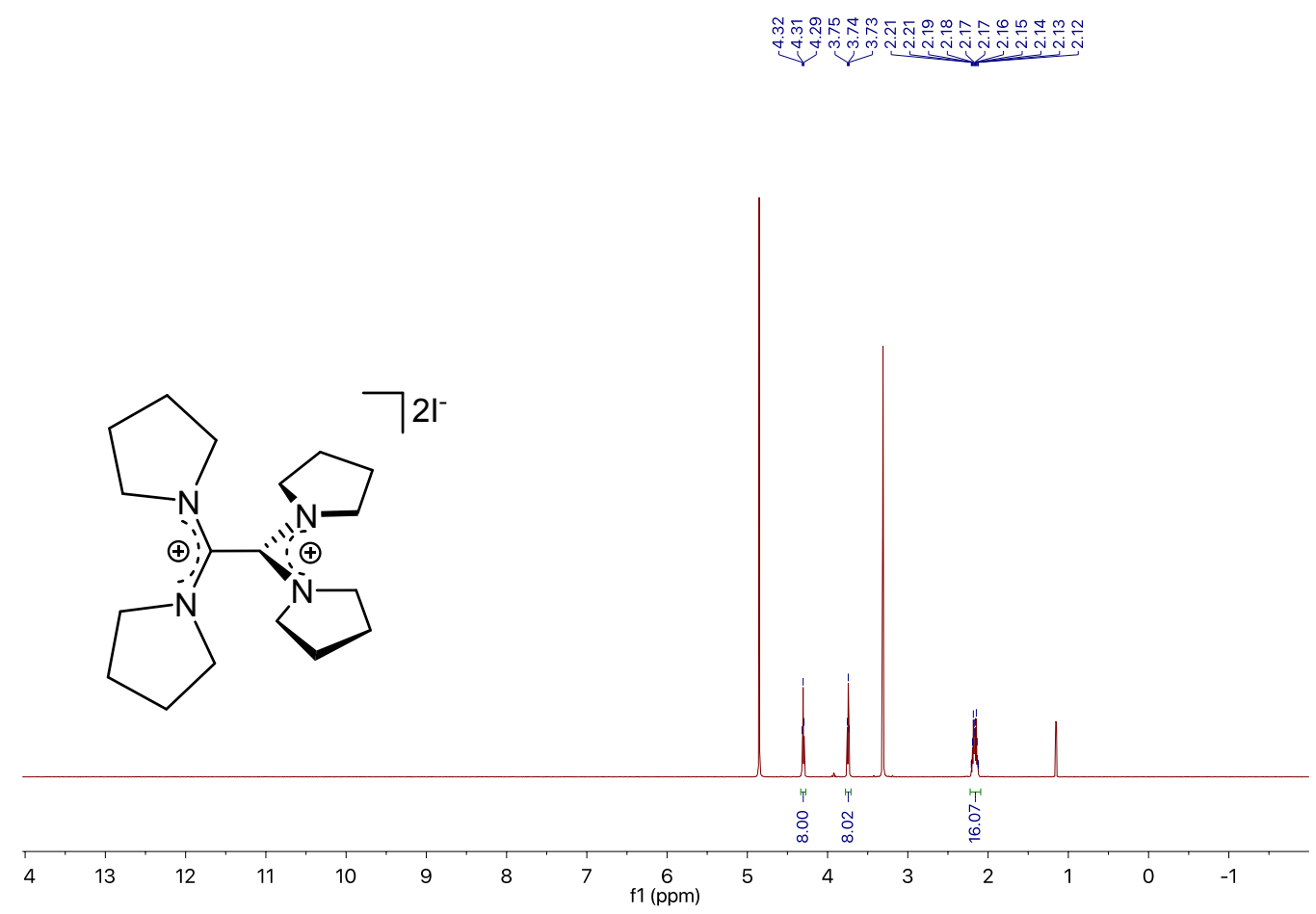

Figure S24. ${ }^{1} \mathrm{H}$ NMR spectrum $\left(600 \mathrm{MHz}\right.$, Methanol- $\left.d_{4}\right)$ of $[\mathrm{TPyE}]^{2+} 2[\mathrm{l}]$ -

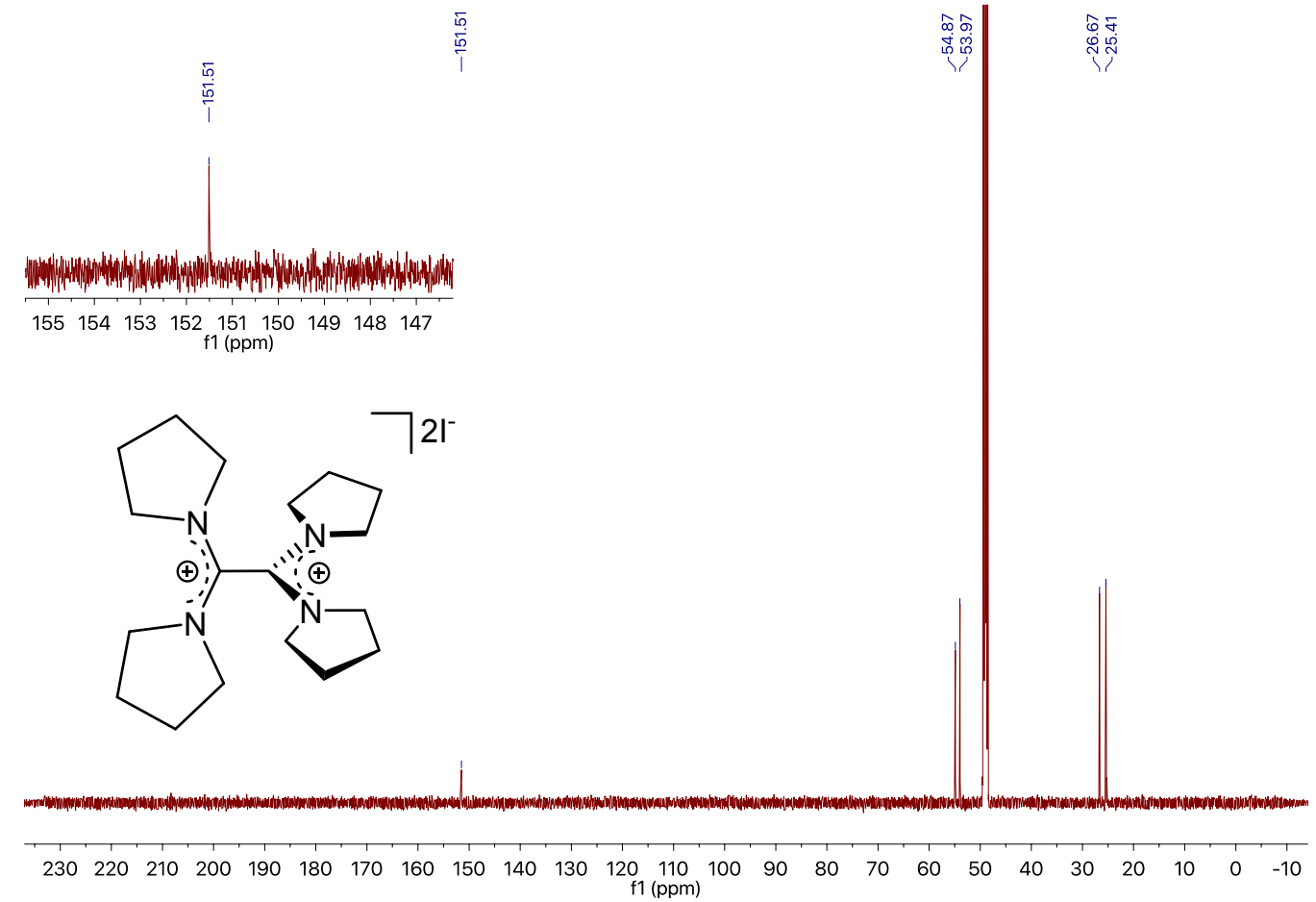

Figure S25. ${ }^{13} \mathrm{C}\left\{{ }^{1} \mathrm{H}\right\}$ NMR spectrum $\left(150 \mathrm{MHz}\right.$, Methanol- $\left.d_{4}\right)$ of $\left.[\mathrm{TPyE}]\right]^{2+2}[[]]$. 


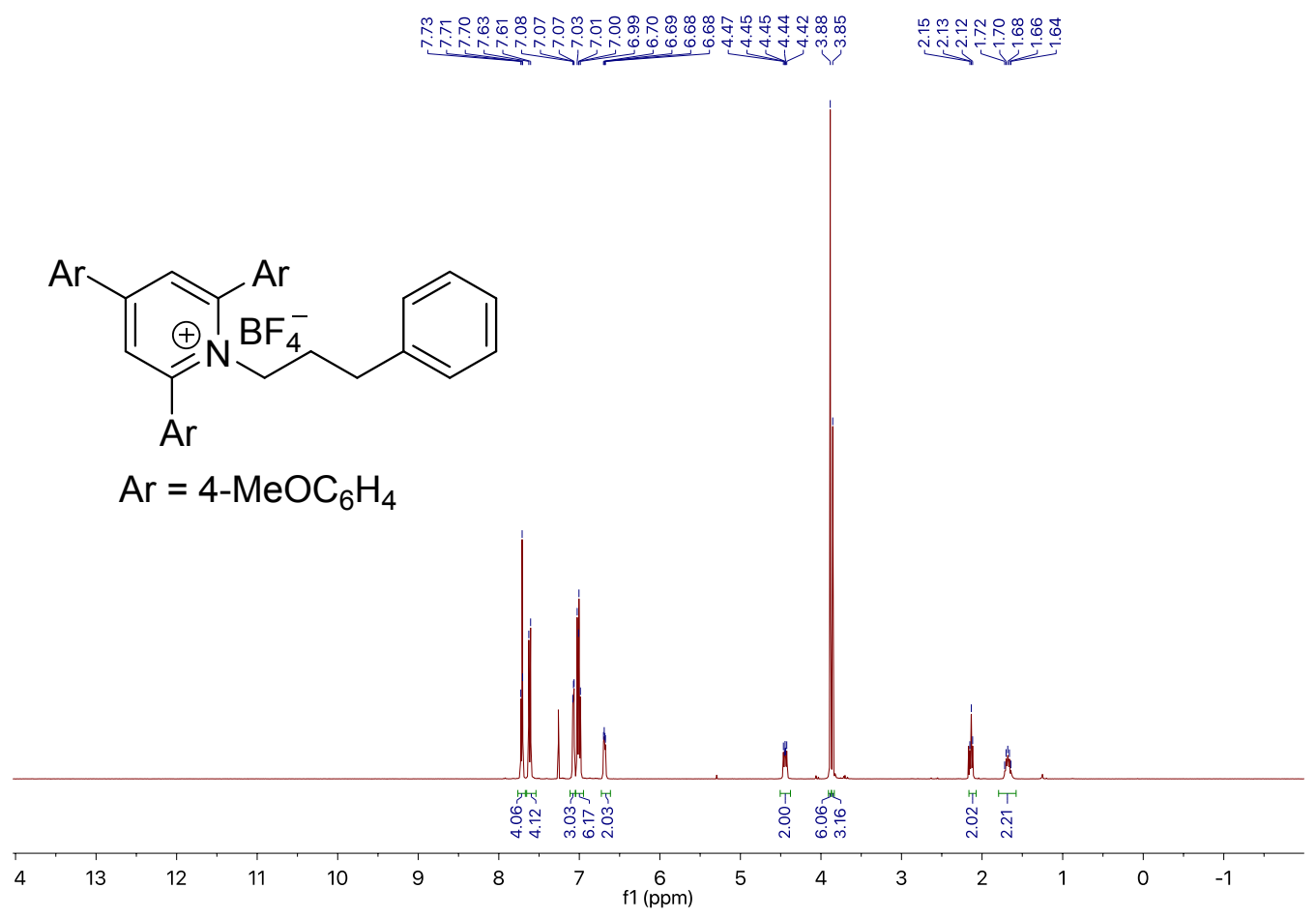

Figure S26. ${ }^{1} \mathrm{H}$ NMR spectrum $\left(400 \mathrm{MHz}, \mathrm{CDCl}_{3}\right)$ of 1.

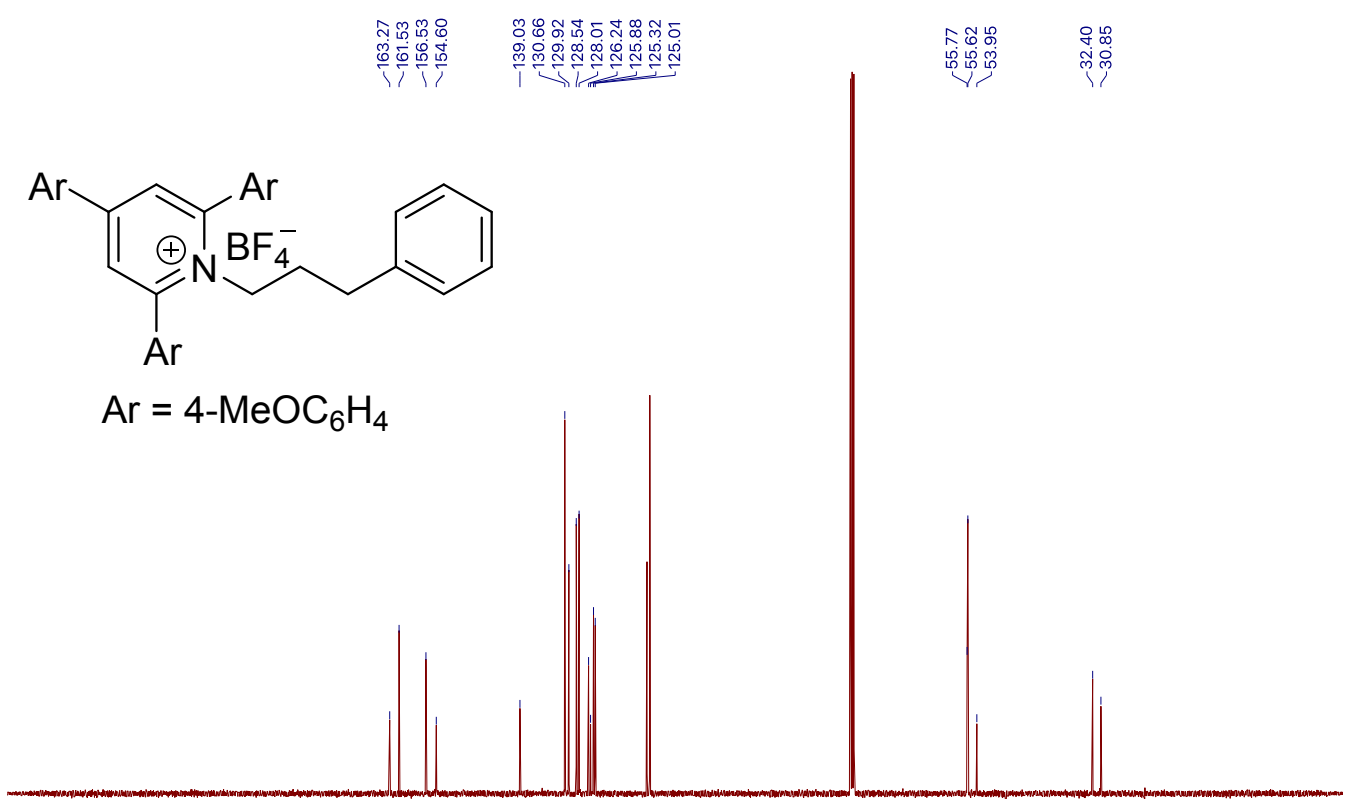

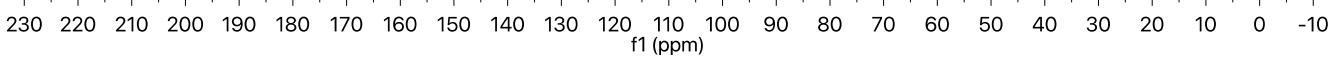

Figure S27. ${ }^{13} \mathrm{C}\left\{{ }^{1} \mathrm{H}\right\}$ NMR spectrum $\left(100 \mathrm{MHz}, \mathrm{CDCl}_{3}\right)$ of 1 . 


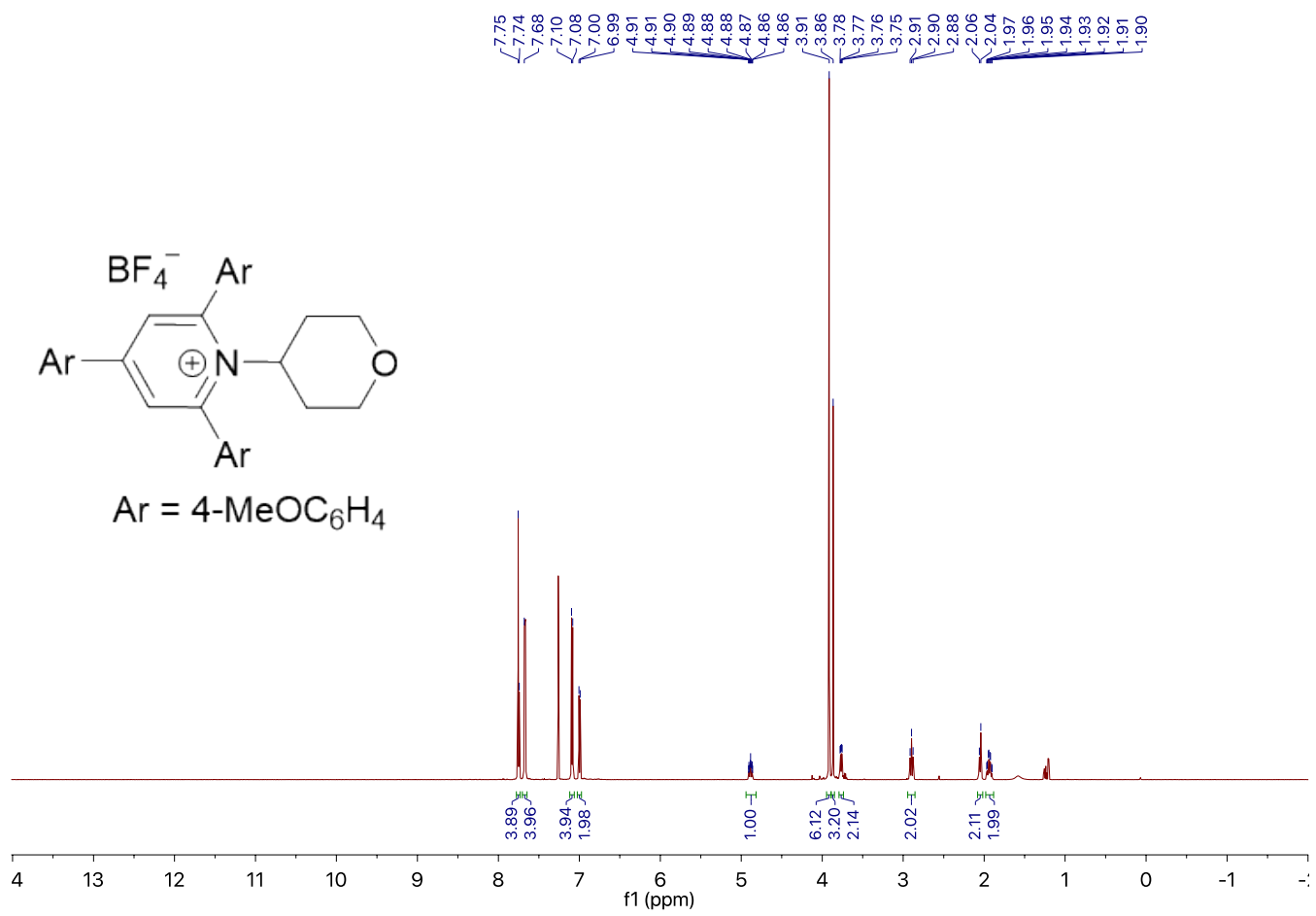

Figure S28. ${ }^{1} \mathrm{H}$ NMR spectrum $\left(600 \mathrm{MHz}, \mathrm{CDCl}_{3}\right)$ of 2.

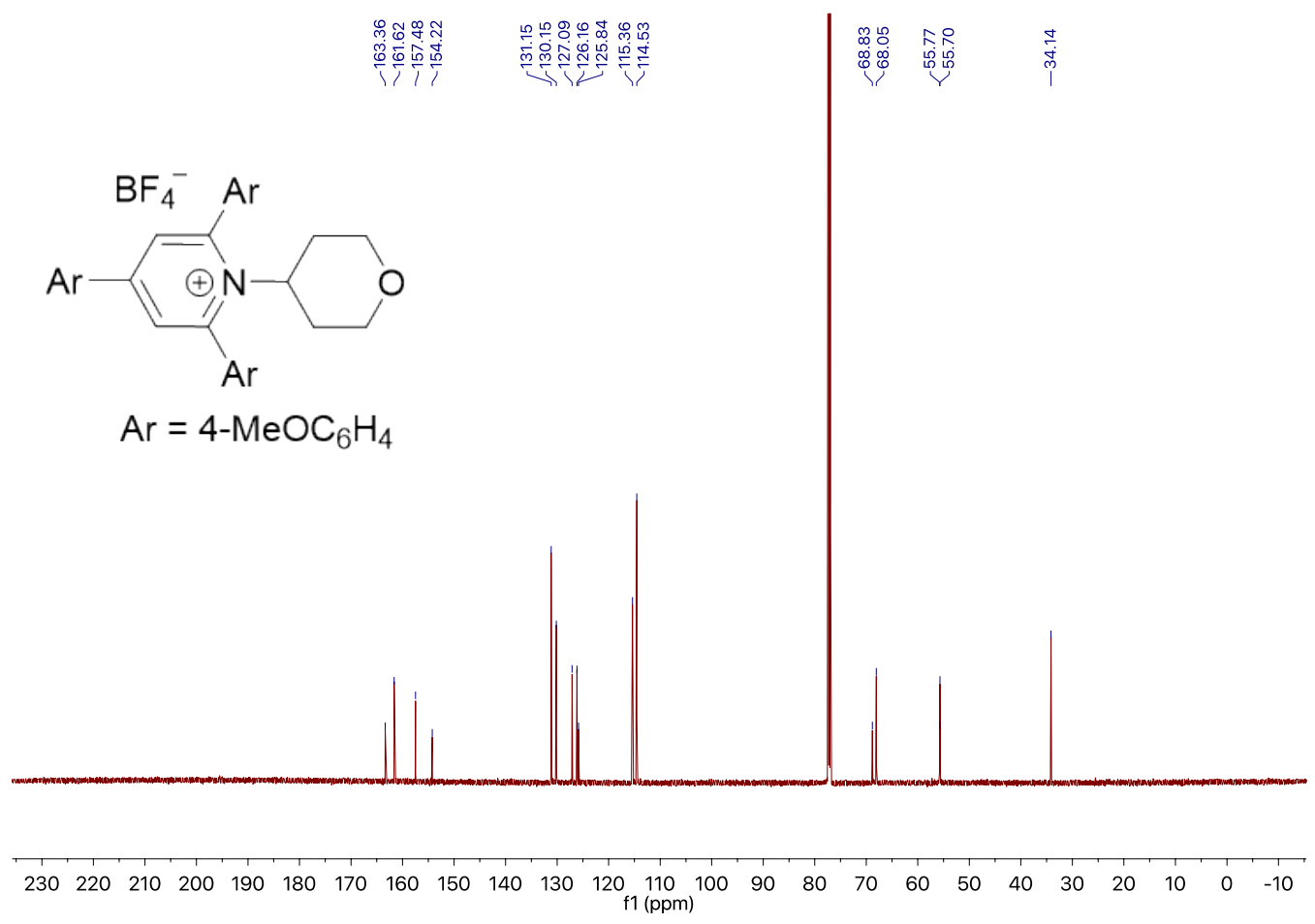

Figure S29. ${ }^{13} \mathrm{C}\left\{{ }^{1} \mathrm{H}\right\}$ NMR spectrum $\left(150 \mathrm{MHz}, \mathrm{CDCl}_{3}\right)$ of 2 . 


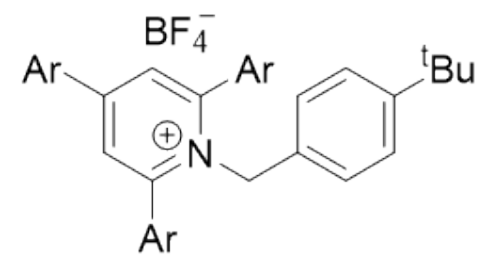

$\mathrm{Ar}=4-\mathrm{MeOC}_{6} \mathrm{H}_{4}$

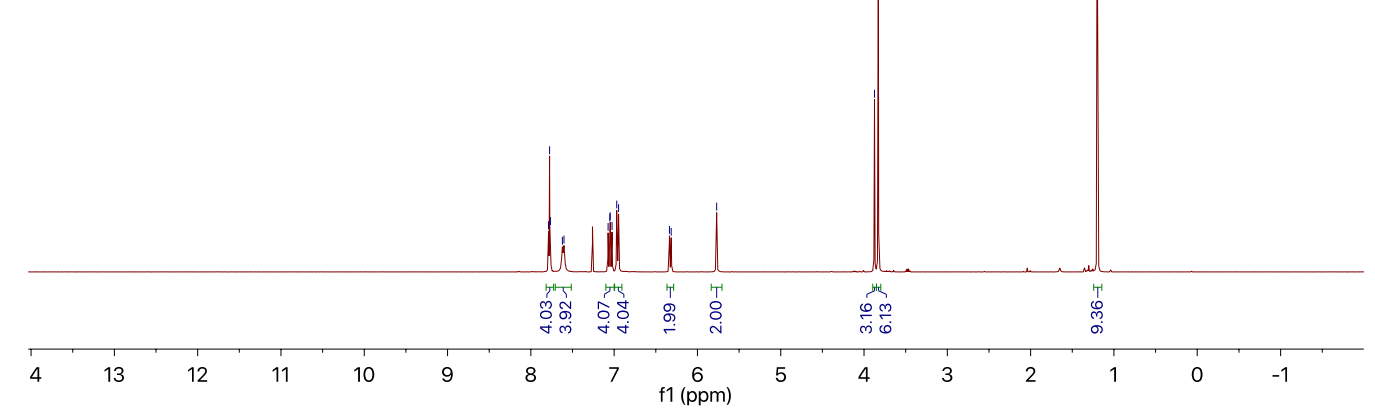

Figure S30. ${ }^{1} \mathrm{H}$ NMR spectrum $\left(400 \mathrm{MHz}, \mathrm{CDCl}_{3}\right)$ of 3.

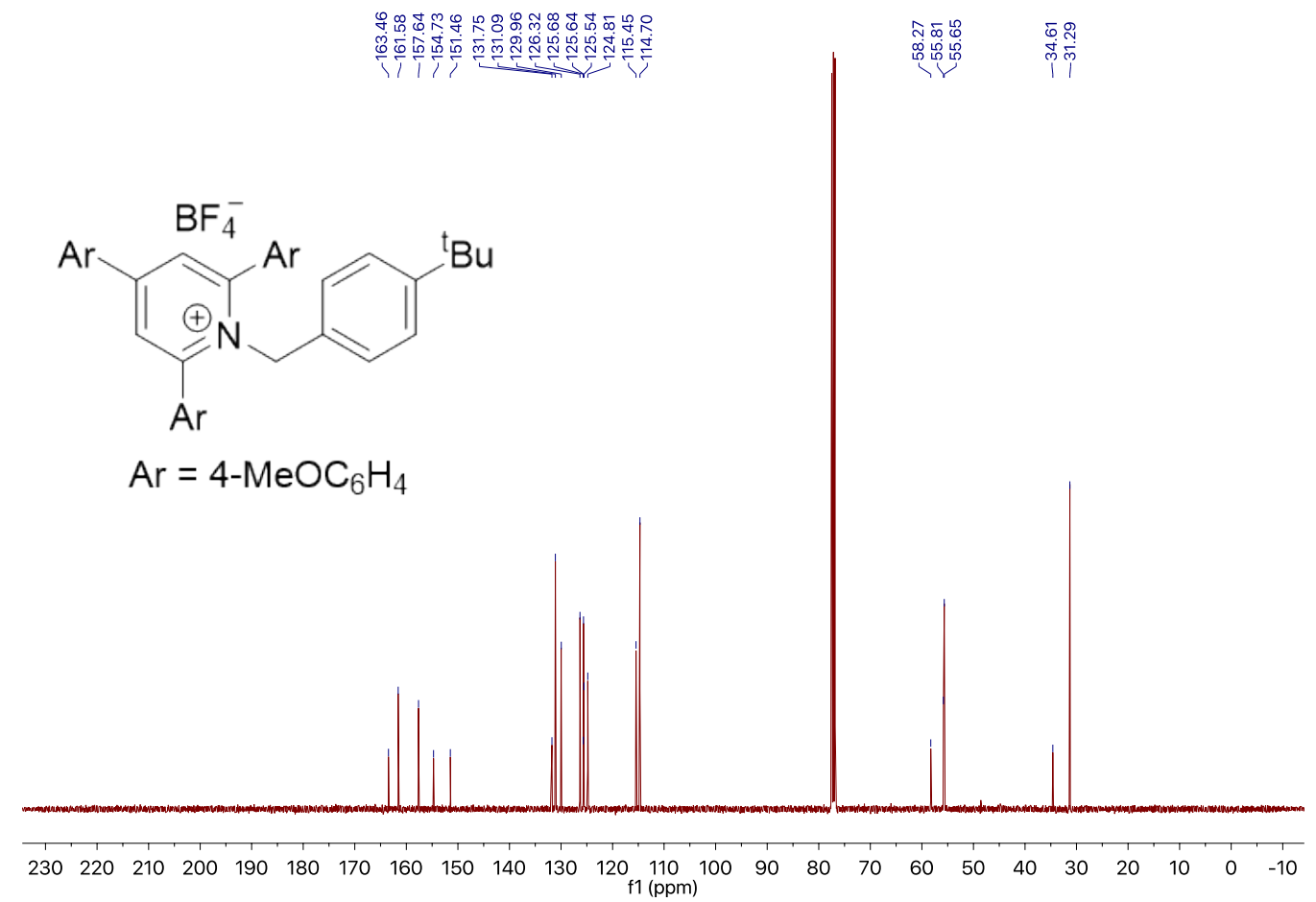

Figure $\mathbf{S} 31 .{ }^{13} \mathrm{C}\left\{{ }^{1} \mathrm{H}\right\}$ NMR spectrum $\left(100 \mathrm{MHz}, \mathrm{CDCl}_{3}\right)$ of 3 . 


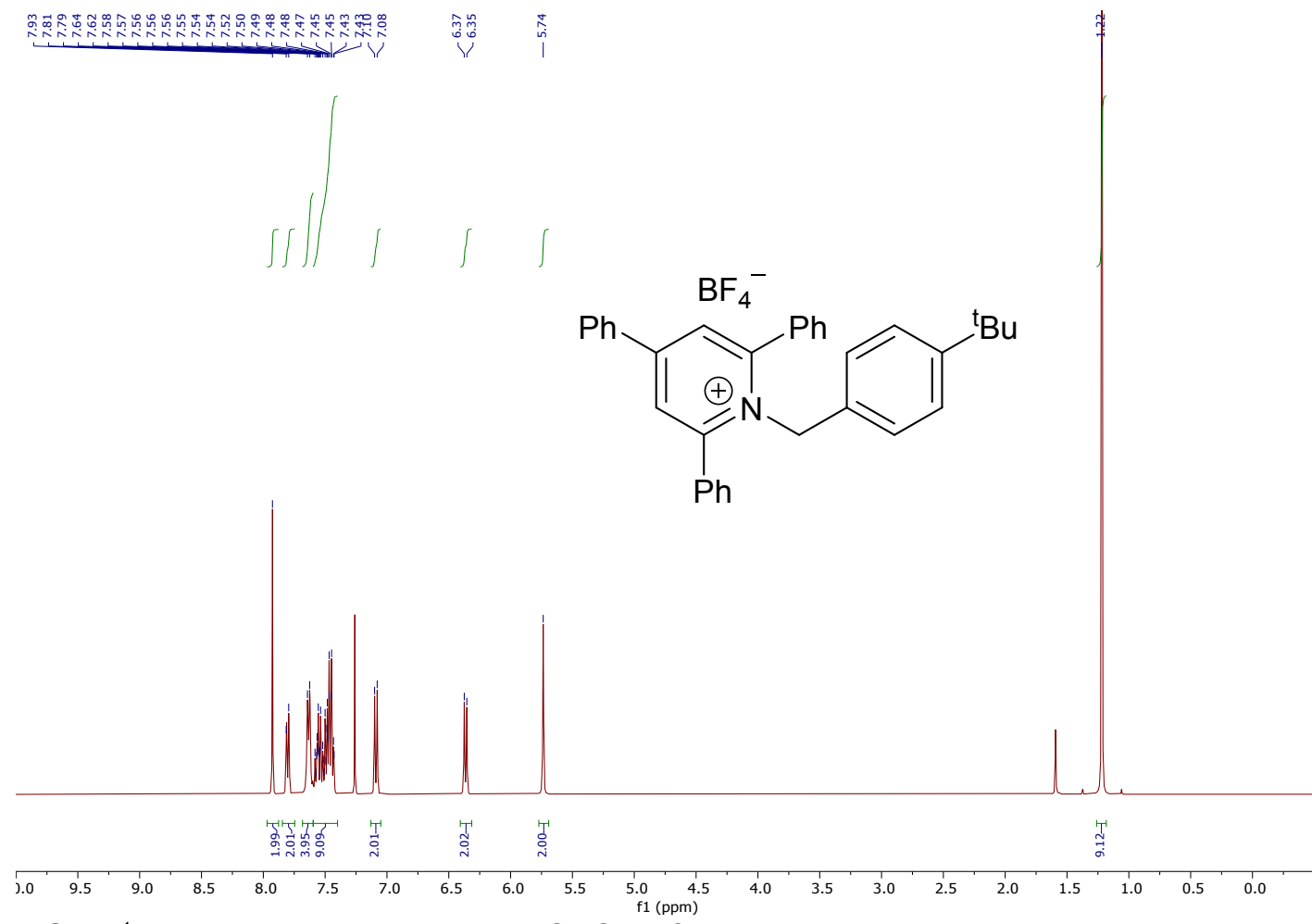

Figure S32. ${ }^{1} \mathrm{H}$ NMR spectrum $\left(400 \mathrm{MHz}, \mathrm{CDCl}_{3}\right)$ of 4 .

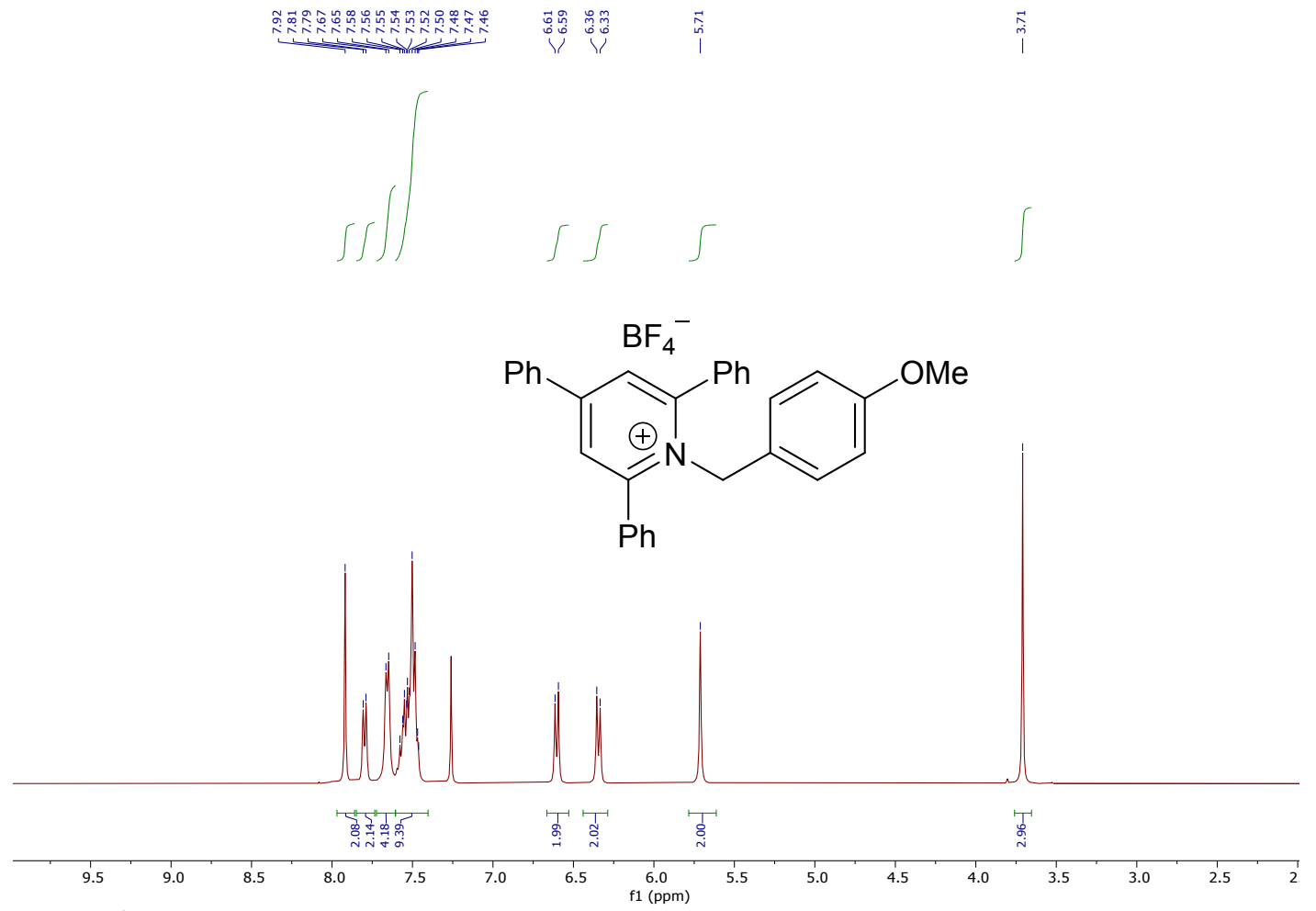

Figure S33. ${ }^{1} \mathrm{H}$ NMR spectrum $\left(400 \mathrm{MHz}, \mathrm{CDCl}_{3}\right)$ of 5 . 


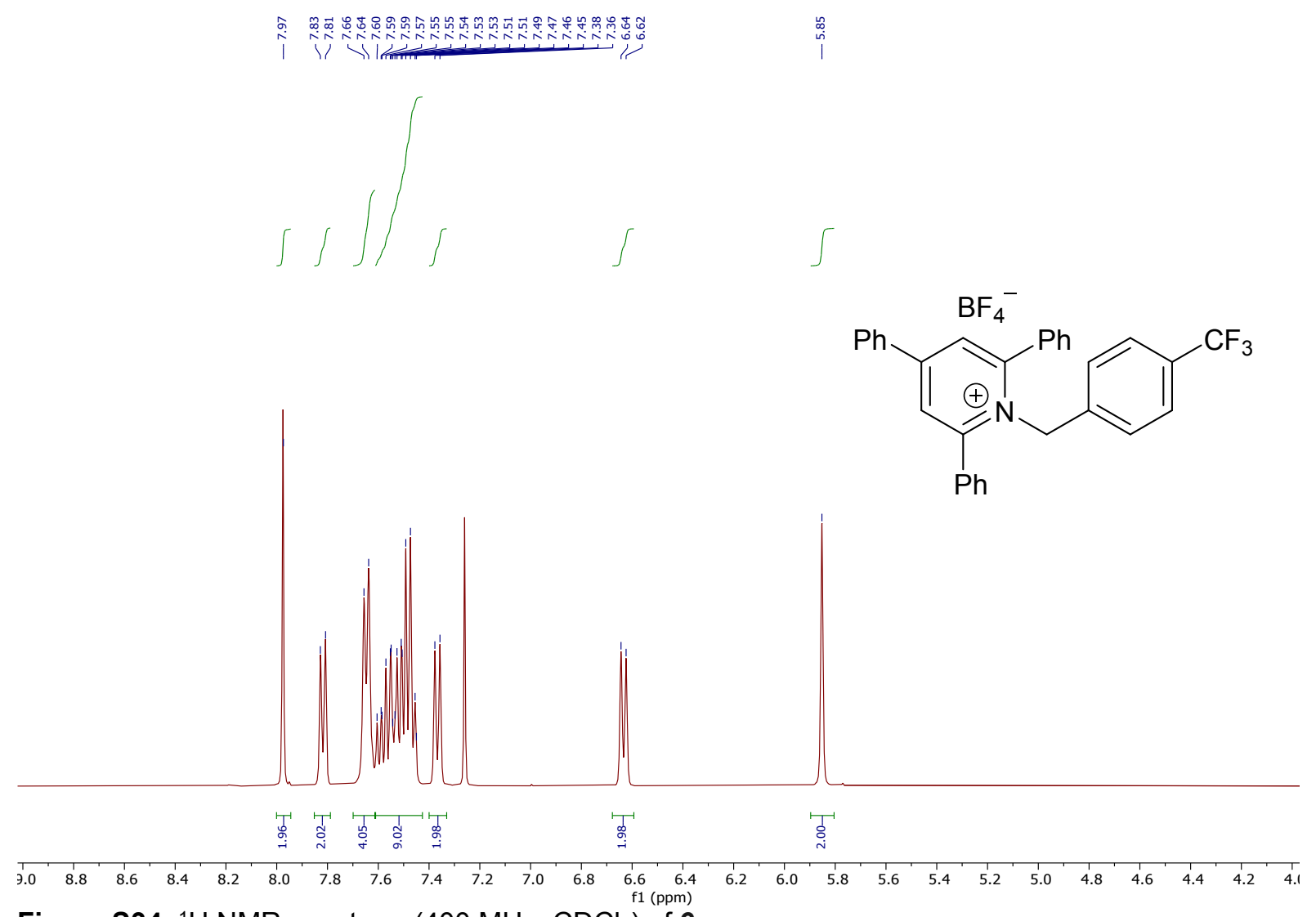

Figure S34. ${ }^{1} \mathrm{H}$ NMR spectrum $\left(400 \mathrm{MHz}, \mathrm{CDCl}_{3}\right)$ of 6 . 
mำำำ

$\stackrel{\varpi}{\prod}$

$1 /$
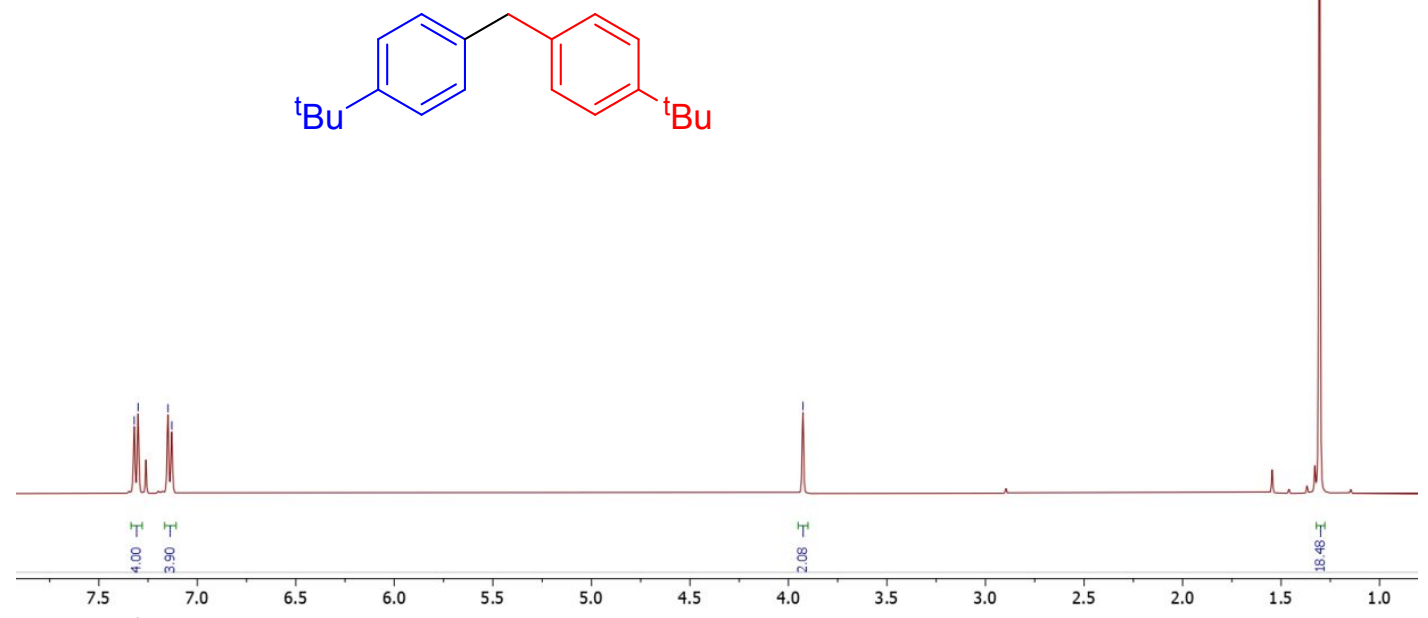

Figure S35. ${ }^{1} \mathrm{H}$ NMR spectrum (400 $\mathrm{MHz}^{\left.-\mathrm{CDCl}_{3}\right)}$ of $\mathbf{9 a}$.

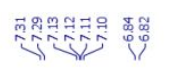

赵爵
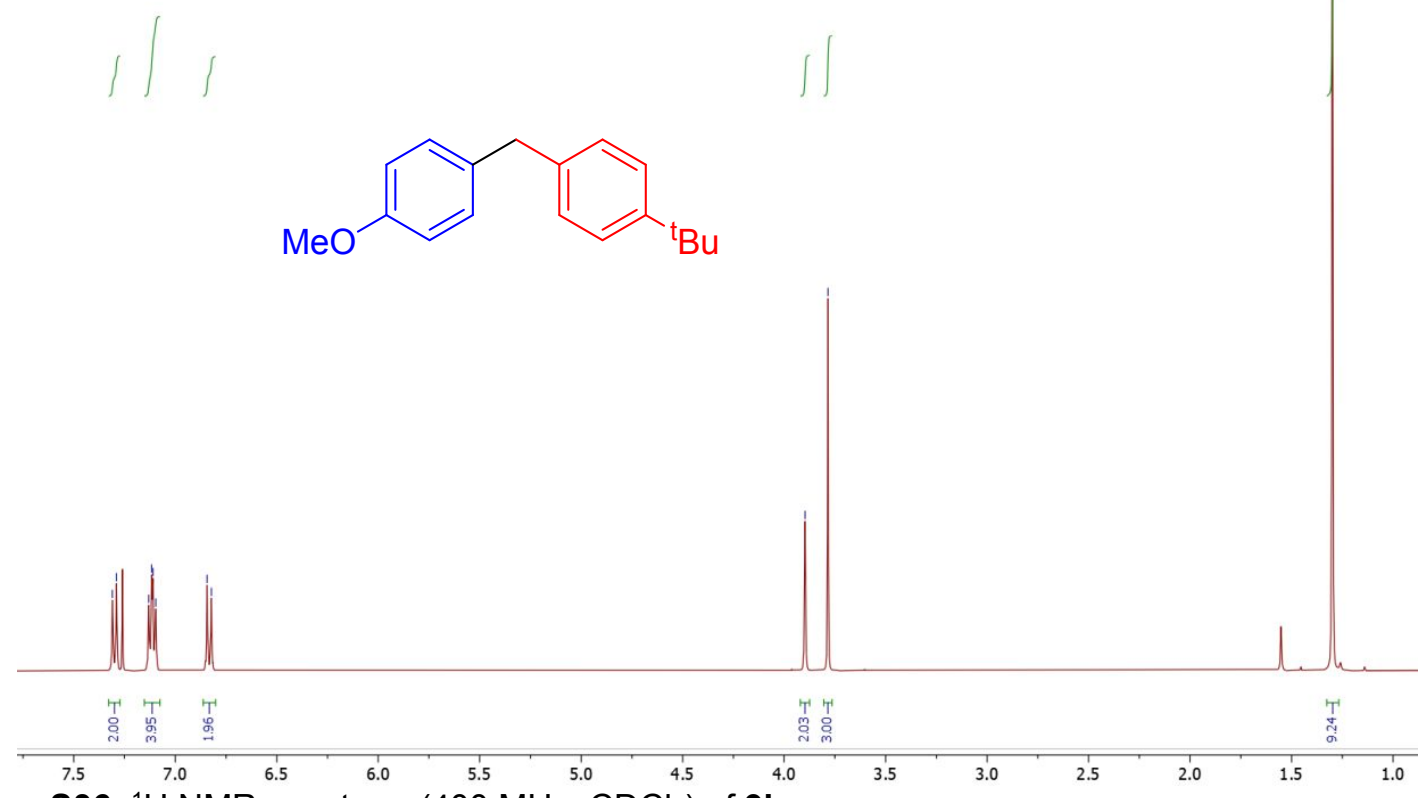

Figure S36. ${ }^{1} \mathrm{H}$ NMR spectrum $\left(400 \mathrm{MHz}, \mathrm{CDCl}_{3}\right)$ of $\mathbf{9 b}$.

S56 


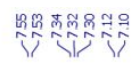<smiles>CC(C)(C)c1ccc(Cc2ccc(C(F)(F)F)cc2)cc1</smiles>

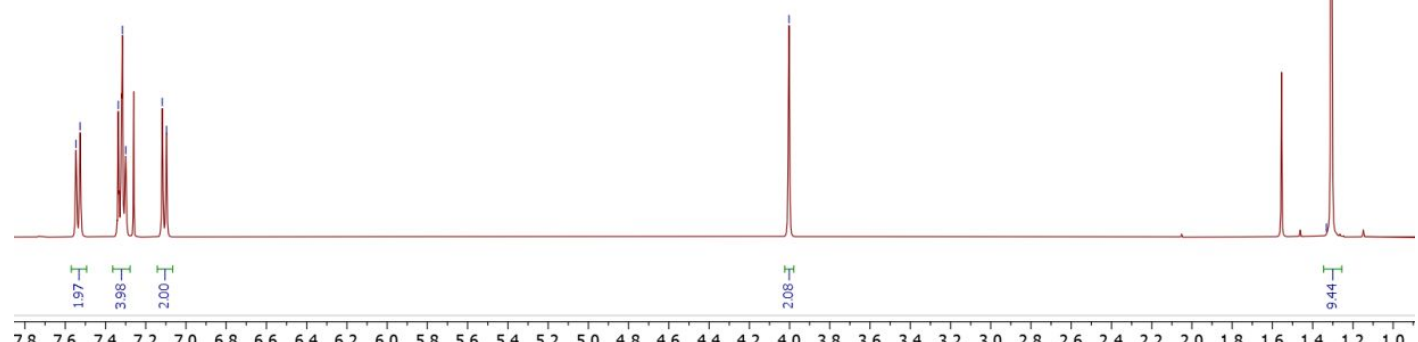

Figure S37. ${ }^{1} \mathrm{H}$ NMR spectrum $\left(400 \mathrm{MHz}, \mathrm{CDCl}_{3}\right)$ of $9 \mathrm{c}$.

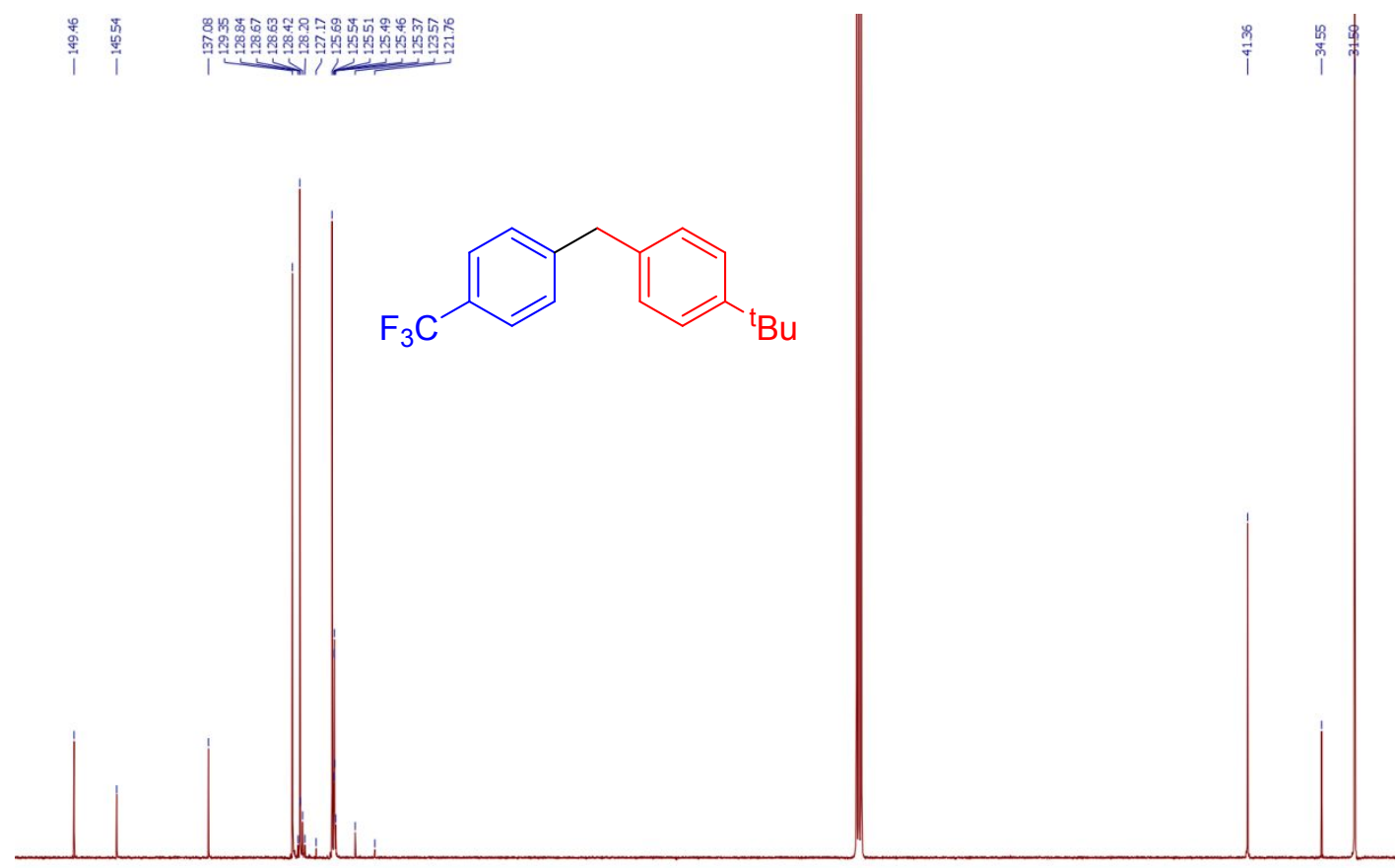

\begin{tabular}{lllllllllllllllllllllllllll}
\hline i5 & 150 & 145 & 140 & 135 & 130 & 125 & 120 & 115 & 110 & 105 & 100 & 95 & 90 & 85 & 80 & 75 & 70 & 65 & 60 & 55 & 50 & 45 & 40 & 35 & 30
\end{tabular} Figure S38. ${ }^{13} \mathrm{C}\left\{{ }^{1} \mathrm{H}\right\}$ NMR spectrum $\left(150 \mathrm{MHz}, \mathrm{CDCl}_{3}\right)$ of 9c. 


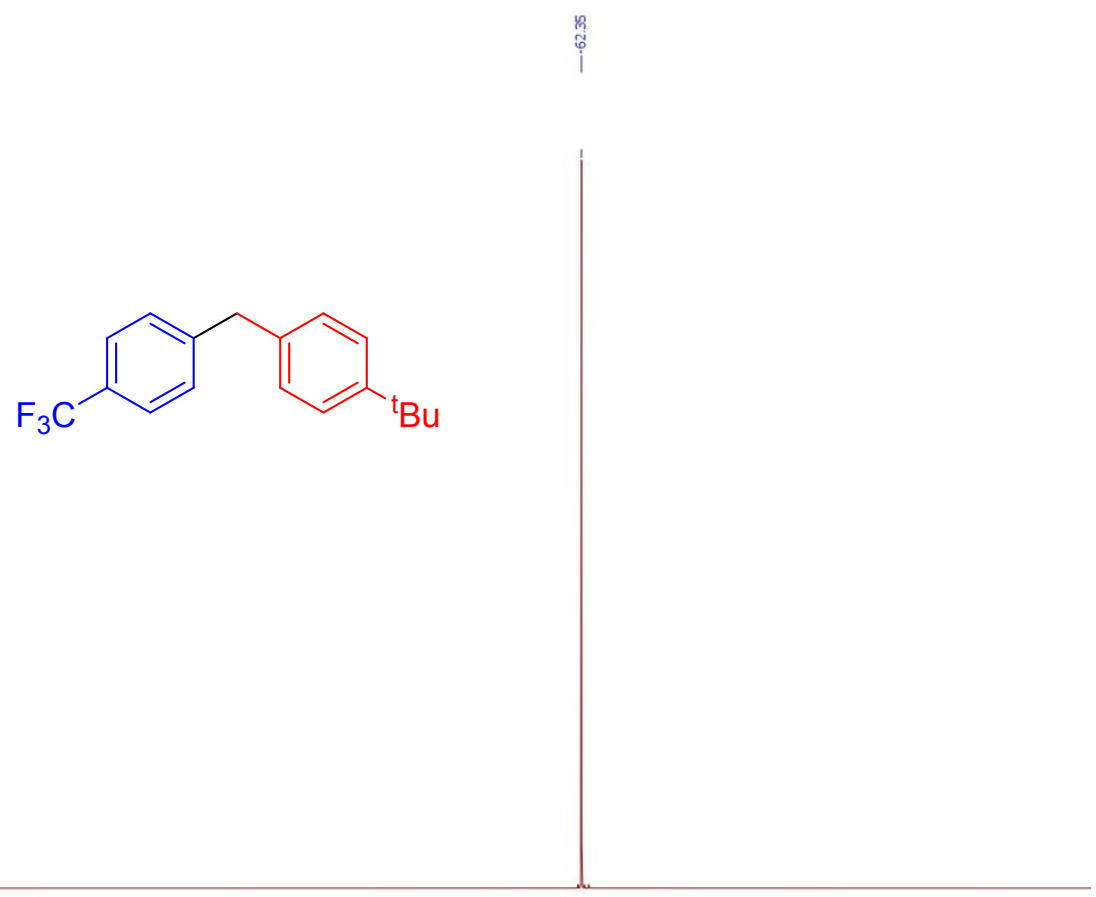

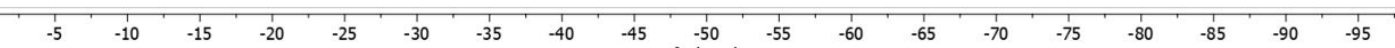

Figure S39. ${ }^{19} \mathrm{~F}\left\{{ }^{1} \mathrm{H}\right\}$ NMR spectrum $\left(376 \mathrm{MHz}, \mathrm{CDCl}_{3}\right)$ of $9 \mathrm{c}$.
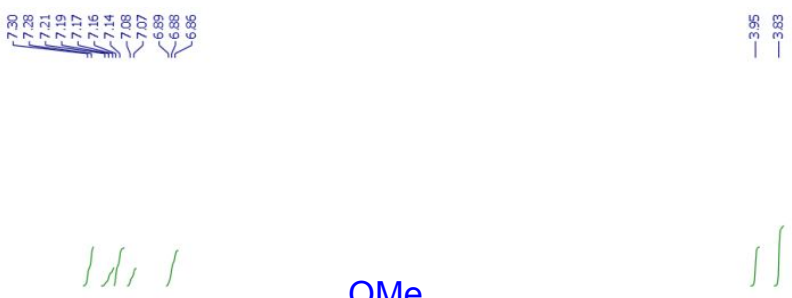<smiles>COc1ccccc1Cc1ccc(C(C)(C)C)cc1</smiles>

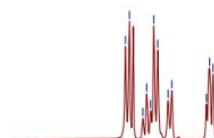

$\mathrm{Bu}$
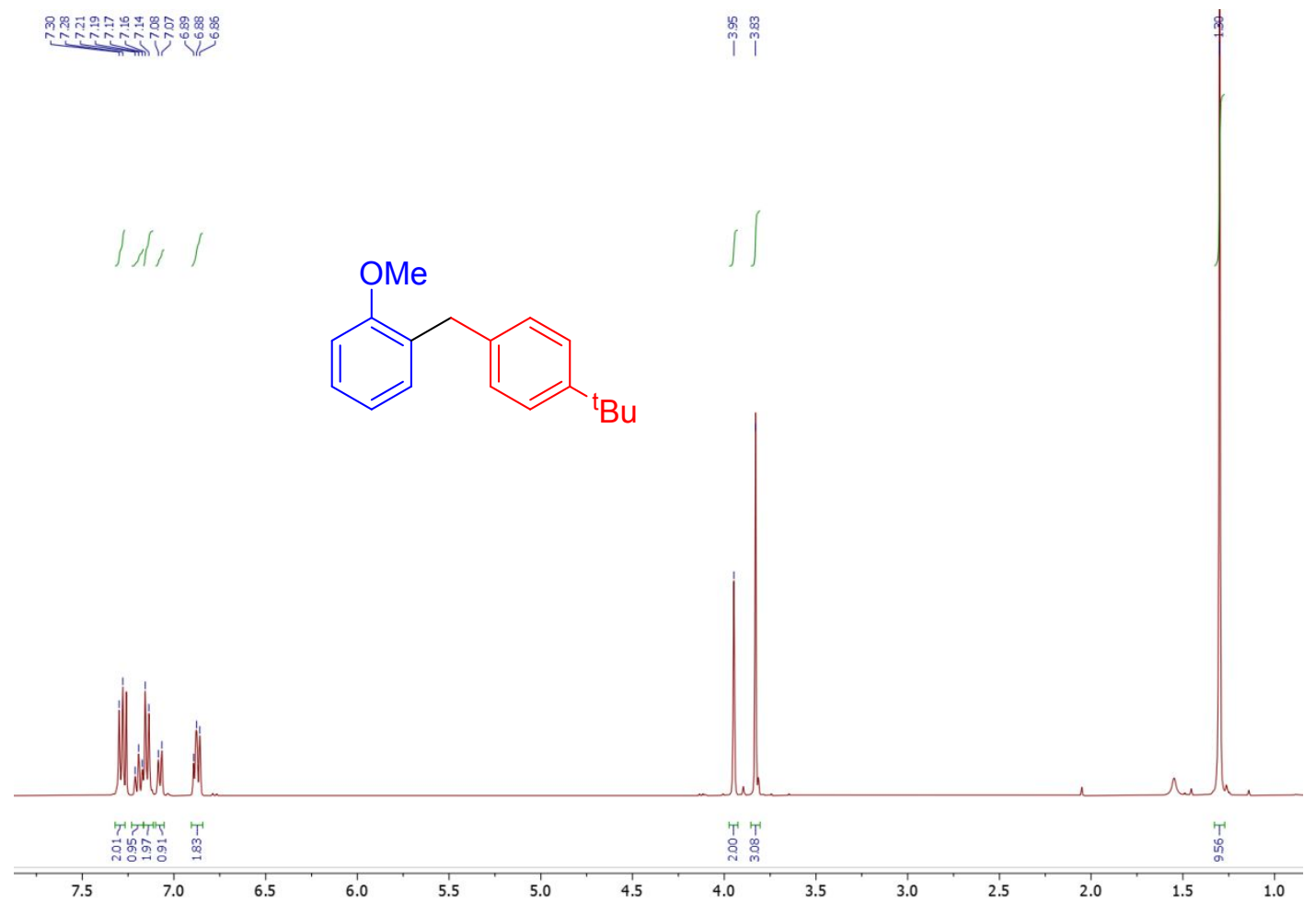

Figure S40. ${ }^{1} \mathrm{H}$ NMR spectrum $\left(400 \mathrm{MHz}, \mathrm{CDCl}_{3}\right)$ of $\mathbf{9 d}$. 


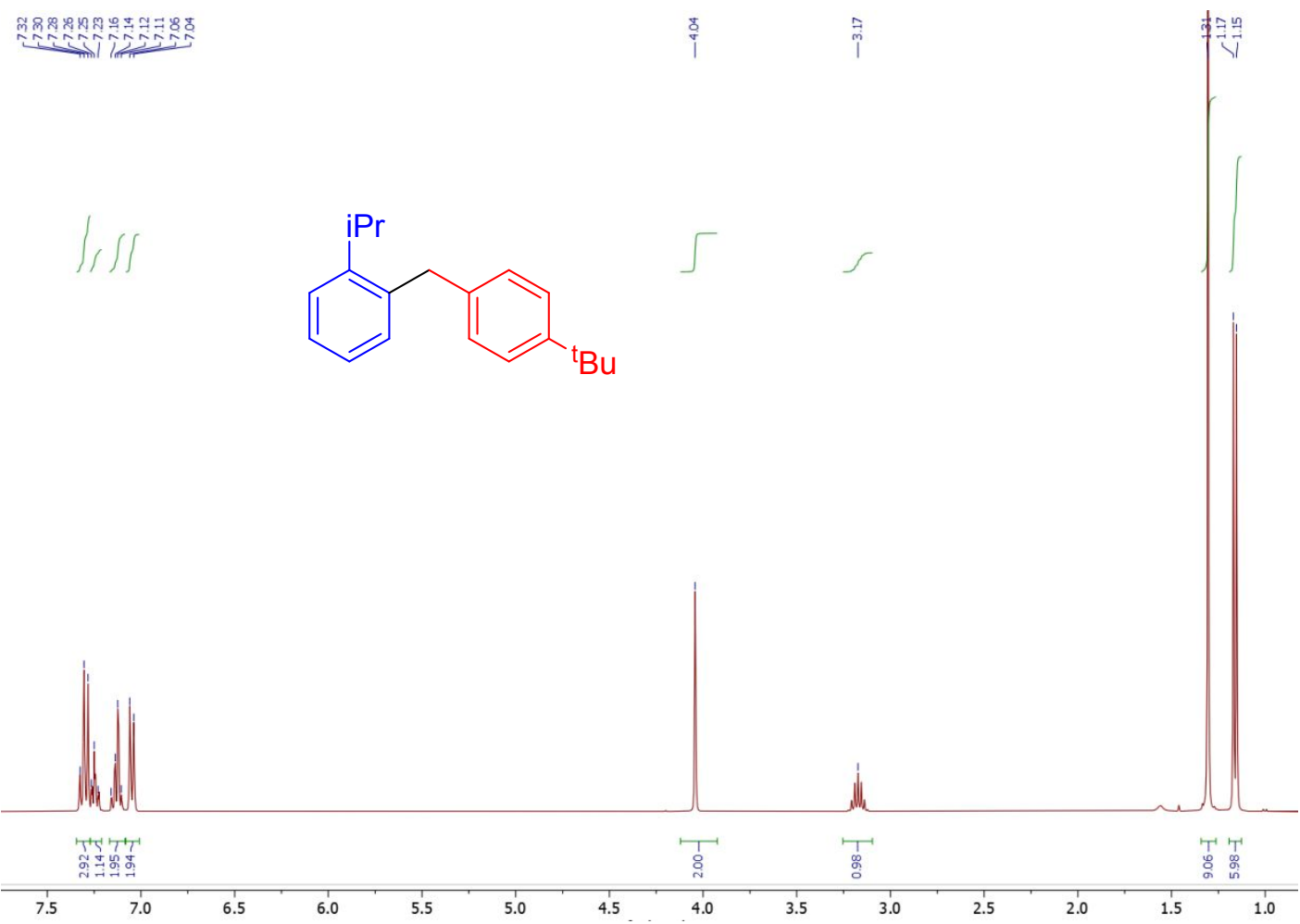

Figure S41. ${ }^{1} \mathrm{H}$ NMR spectrum $\left(400 \mathrm{MHz}, \mathrm{CDCl}_{3}\right)$ of $9 \mathbf{e}$.

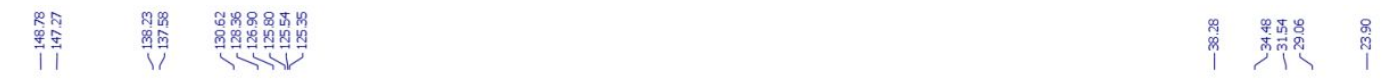<smiles>CC(C)c1ccc(Cc2ccccc2C(C)(C)C)cc1</smiles>
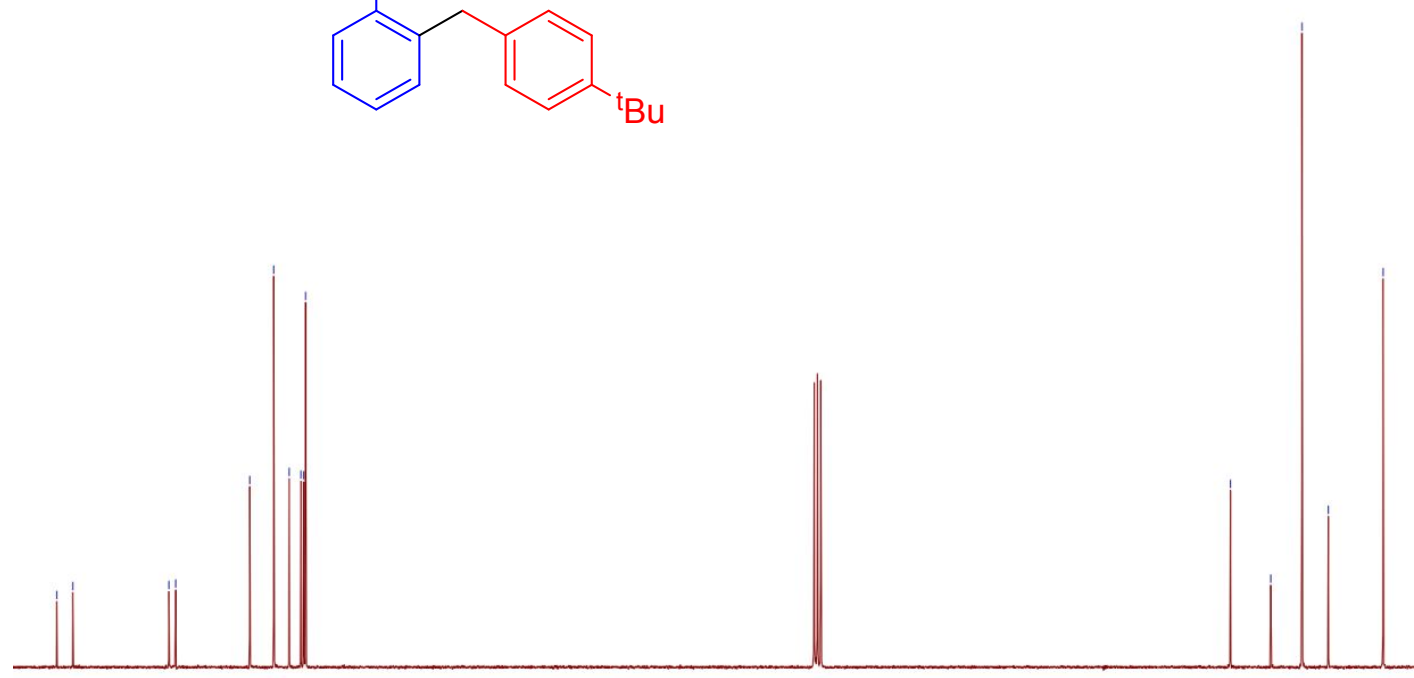

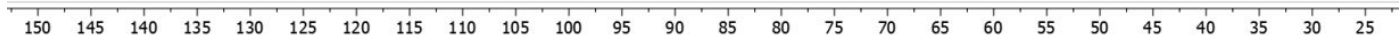
Figure S42. ${ }^{13} \mathrm{C}\left\{{ }^{1} \mathrm{H}\right\}$ NMR spectrum $\left(150 \mathrm{MHz}, \mathrm{CDCl}_{3}\right)$ of $\mathbf{9 e}$. 


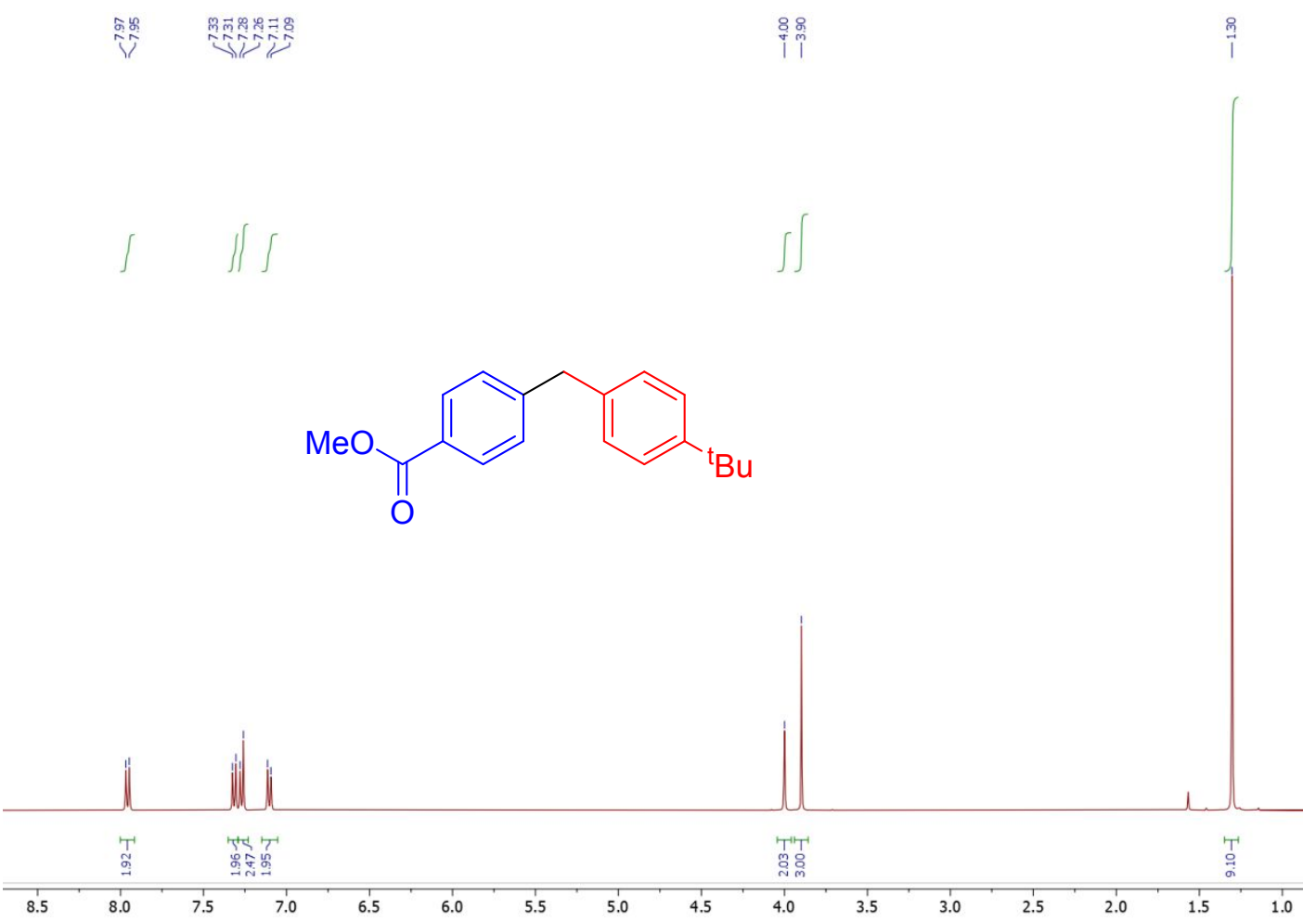

Figure S43. ${ }^{1} \mathrm{H}$ NMR spectrum $\left(400 \mathrm{MHz}, \mathrm{CDCl}_{3}\right)$ of 9.
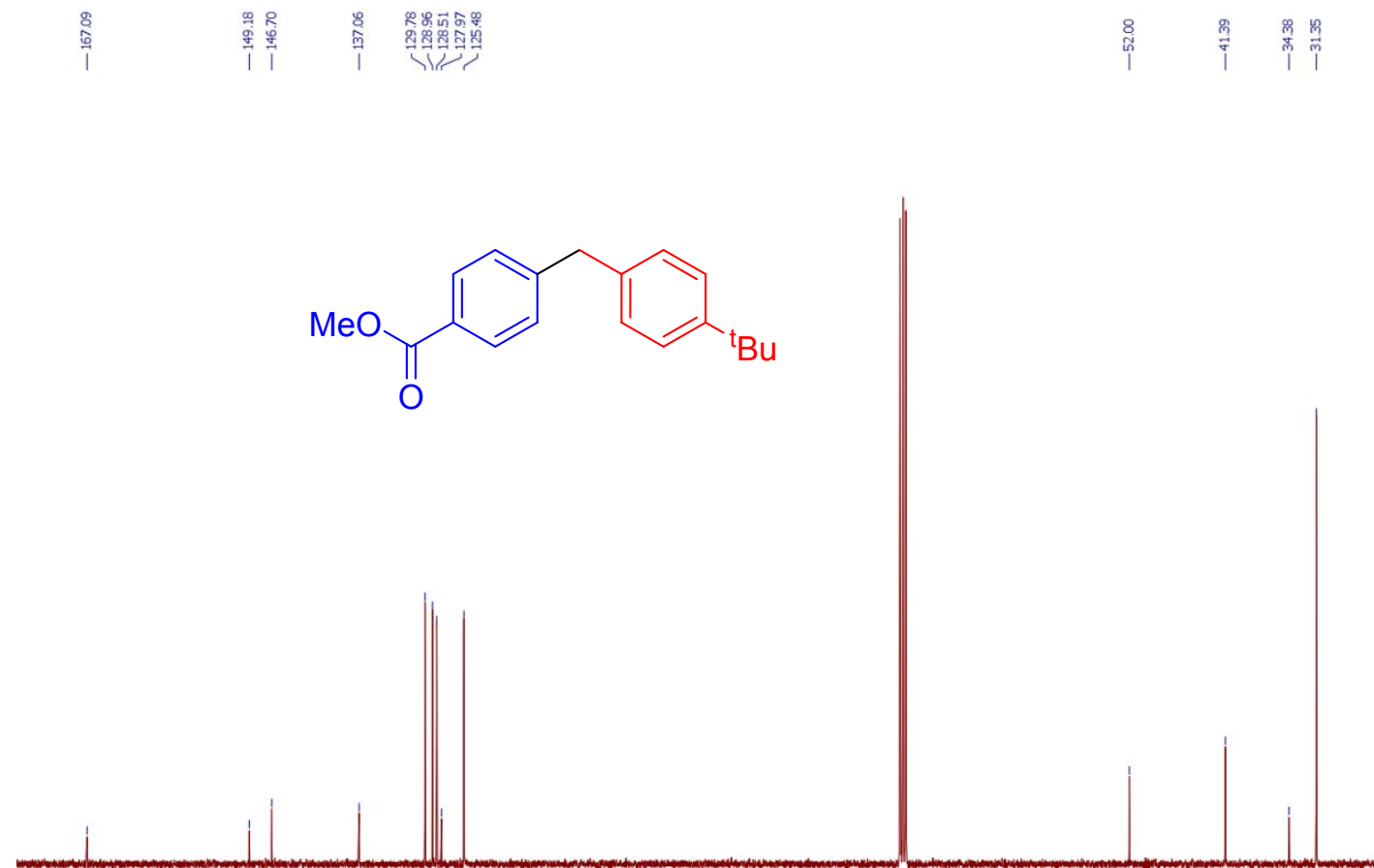

Figure S44. ${ }^{13} \mathrm{C}\left\{{ }^{17} \mathrm{H}\right\}$ NMR spectrum $\left(150 \mathrm{MHz}, \mathrm{CDCl}_{3}\right)$ of $\mathbf{9 f}$. 


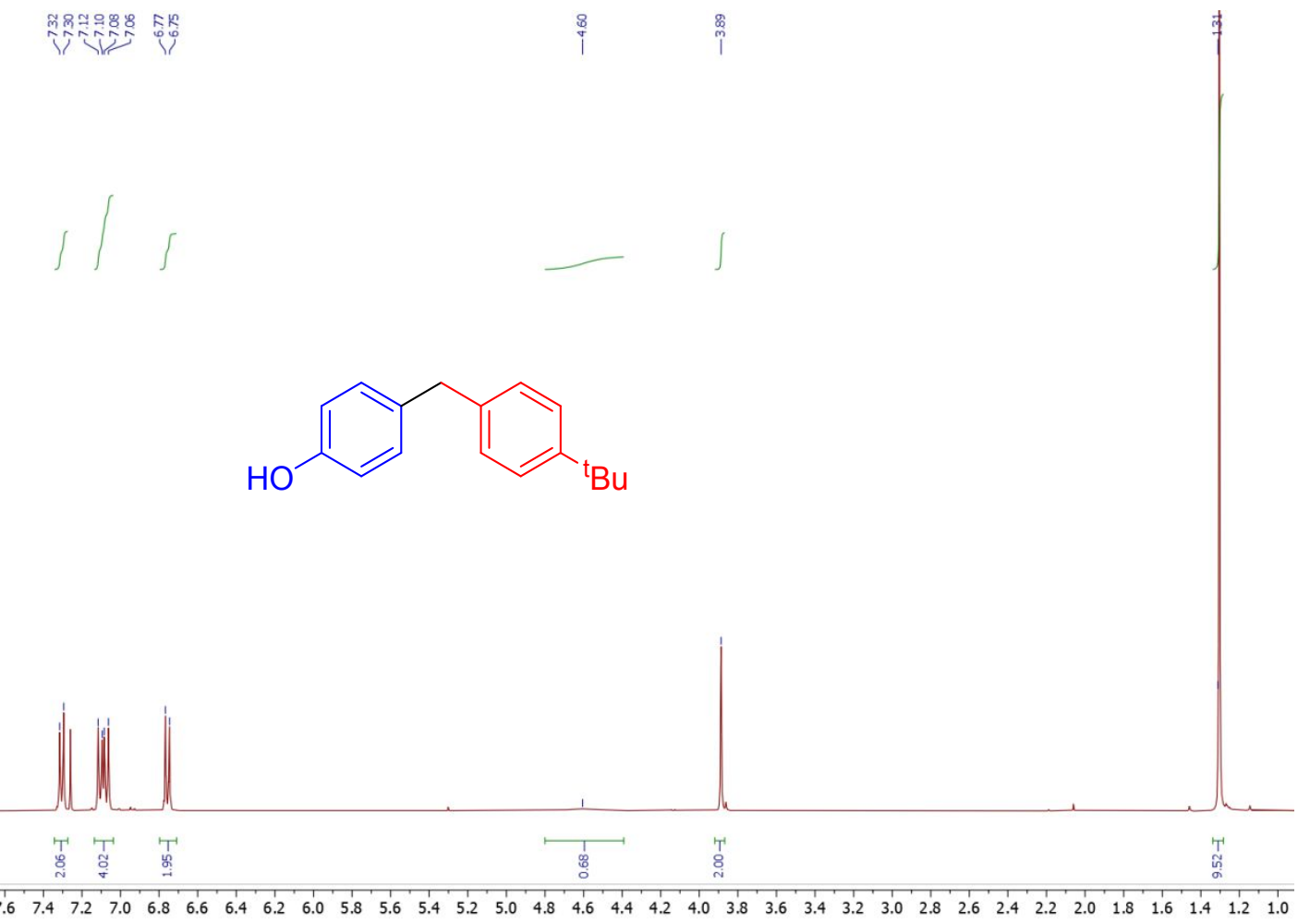

Figure S45. ${ }^{1} \mathrm{H}$ NMR spectrum $\left(400 \mathrm{MHz}, \mathrm{CDCl}_{3}\right)$ of $\mathbf{9 g}$. 

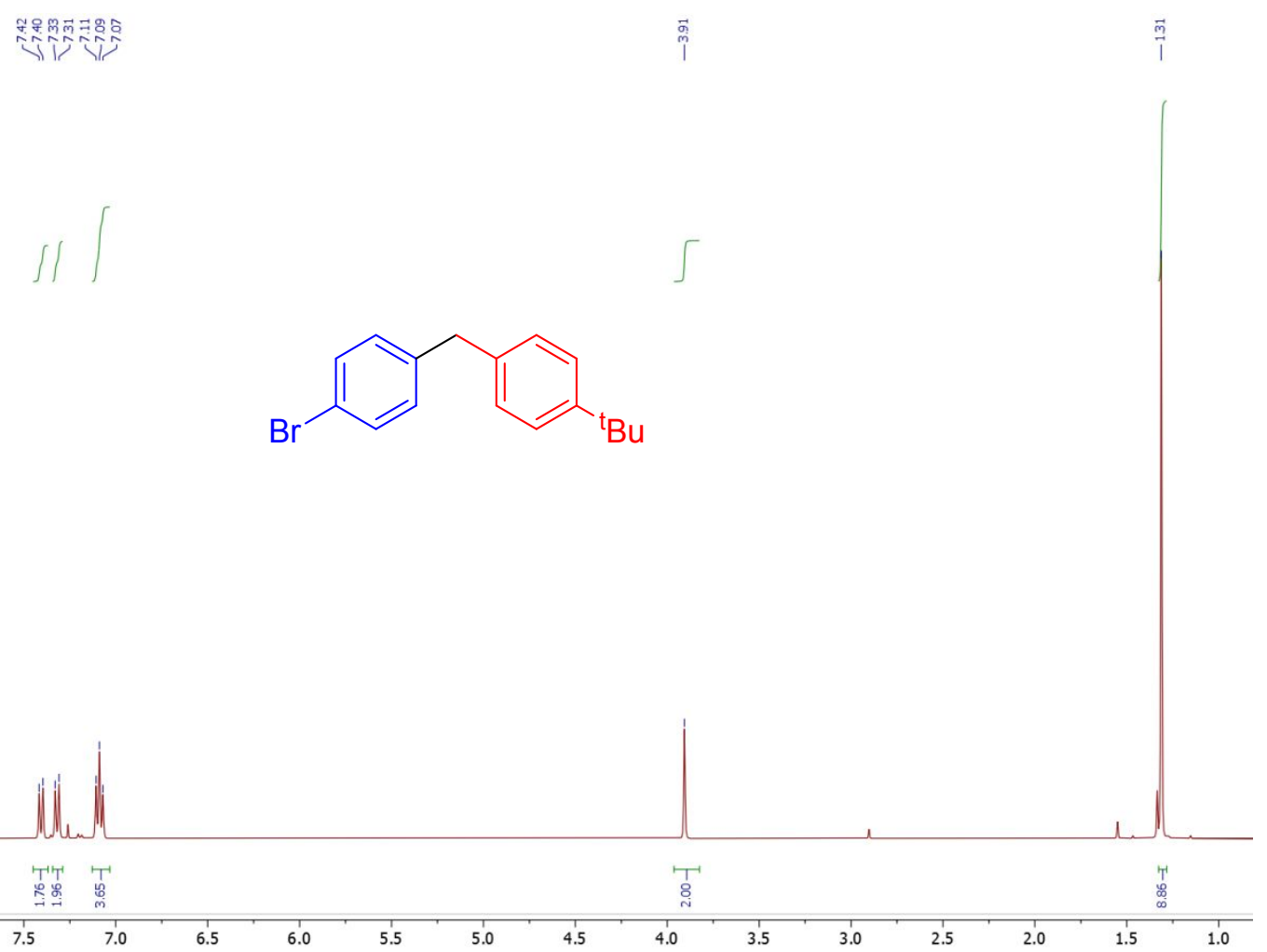

Figure S46. ${ }^{1} \mathrm{H}$ NMR spectrum $\left(400 \mathrm{MHz}, \mathrm{CDCl}_{3}\right)$ of $\mathbf{9 h}$.

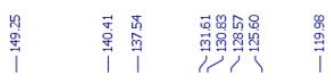

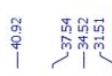<smiles>CC(C)(C)c1ccc(Cc2ccc(Br)cc2)cc1</smiles>
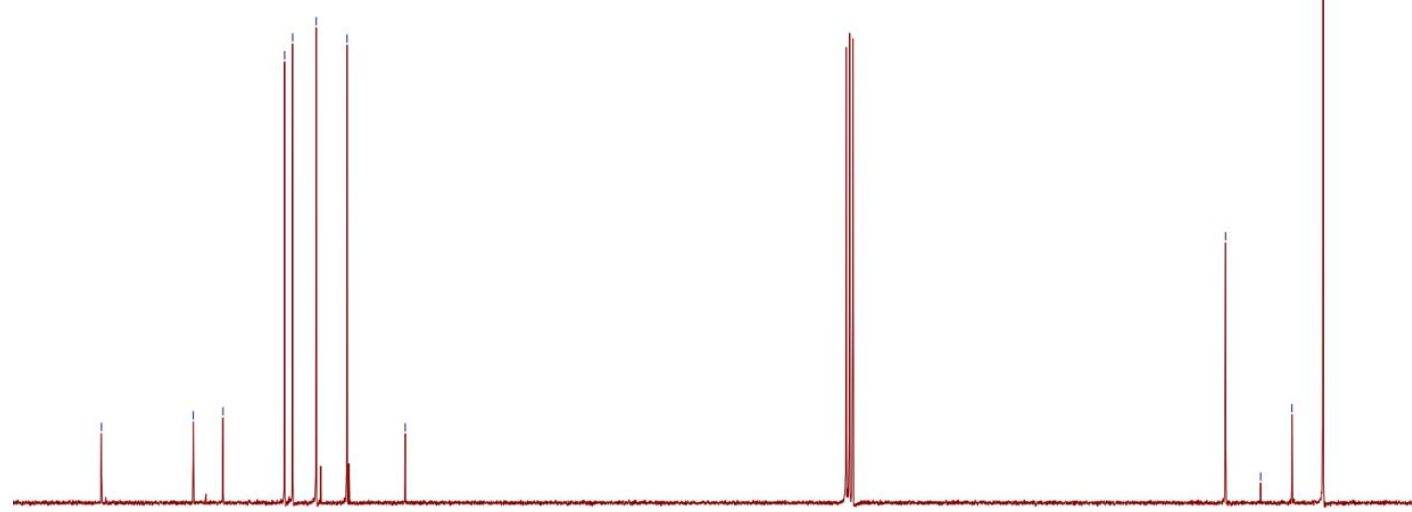

\begin{tabular}{llllllllllllllllllllllllllllllll}
\hline & 155 & 150 & 145 & 140 & 135 & 130 & 125 & 120 & 115 & 110 & 105 & 100 & 95 & 90 & 85 & 80 & 75 & 70 & 65 & 60 & 55 & 50 & 45 & 40 & 35 & 30 & 25
\end{tabular} Figure $\mathbf{S} 47 .{ }^{13} \mathrm{C}\left\{{ }^{1} \mathrm{H}\right\} \mathrm{NMR}$ spectrum $\left(150 \mathrm{MHz}, \mathrm{CDCl}_{3}\right)$ of $\mathbf{9 h}$. 


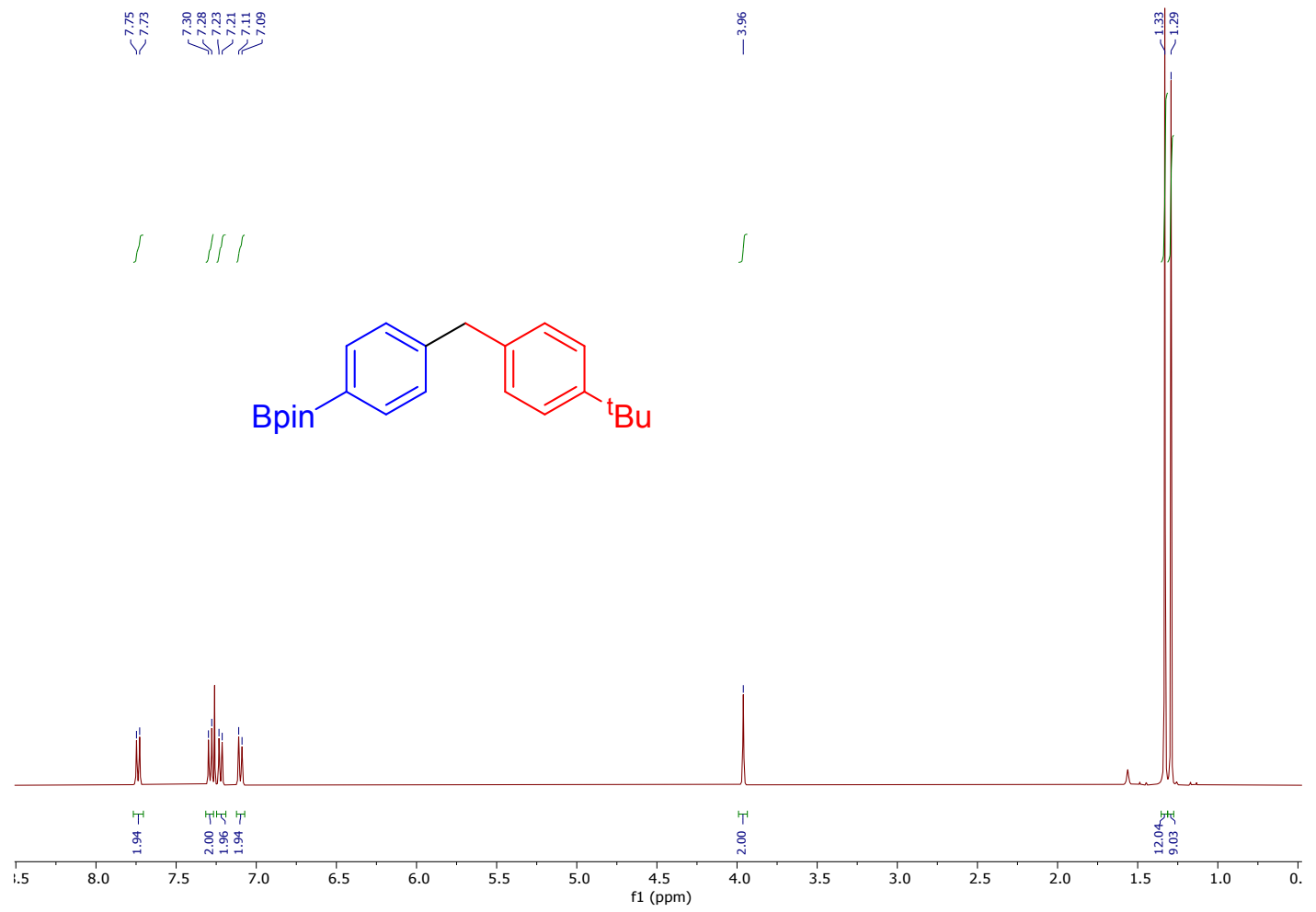

Figure S48. ${ }^{1} \mathrm{H}$ NMR spectrum $\left(400 \mathrm{MHz}, \mathrm{CDCl}_{3}\right)$ of $\mathbf{9 i}$.

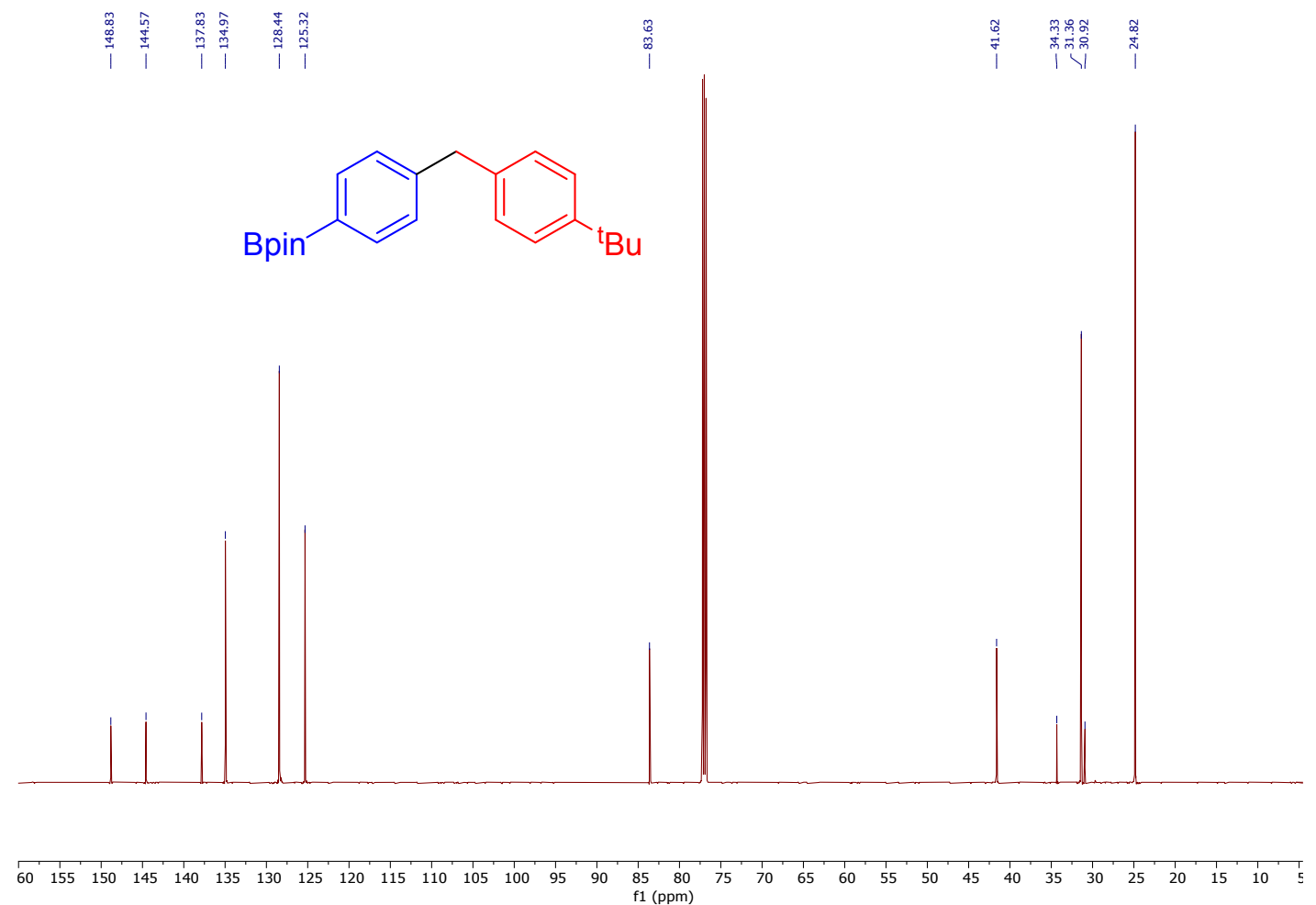

Figure S49. ${ }^{13} \mathrm{C}\left\{{ }^{1} \mathrm{H}\right\}$ NMR spectrum $\left(150 \mathrm{MHz}, \mathrm{CDCl}_{3}\right)$ of $9 \mathbf{i}$. 


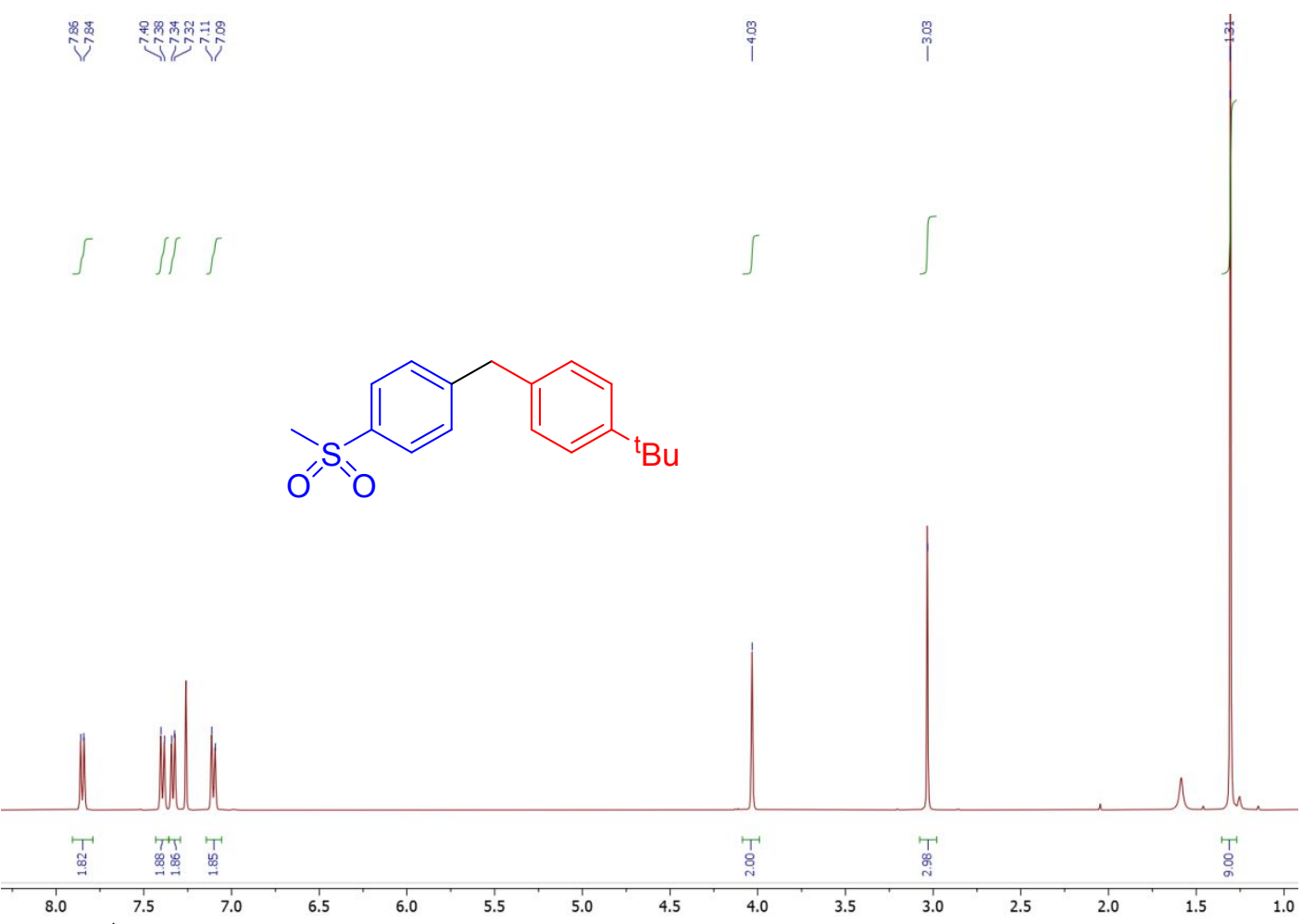

Figure S50. ${ }^{1} \mathrm{H}$ NMR spectrum $\left(400 \mathrm{MHz}, \mathrm{CDCl}_{3}\right)$ of $\mathbf{9 j}$.

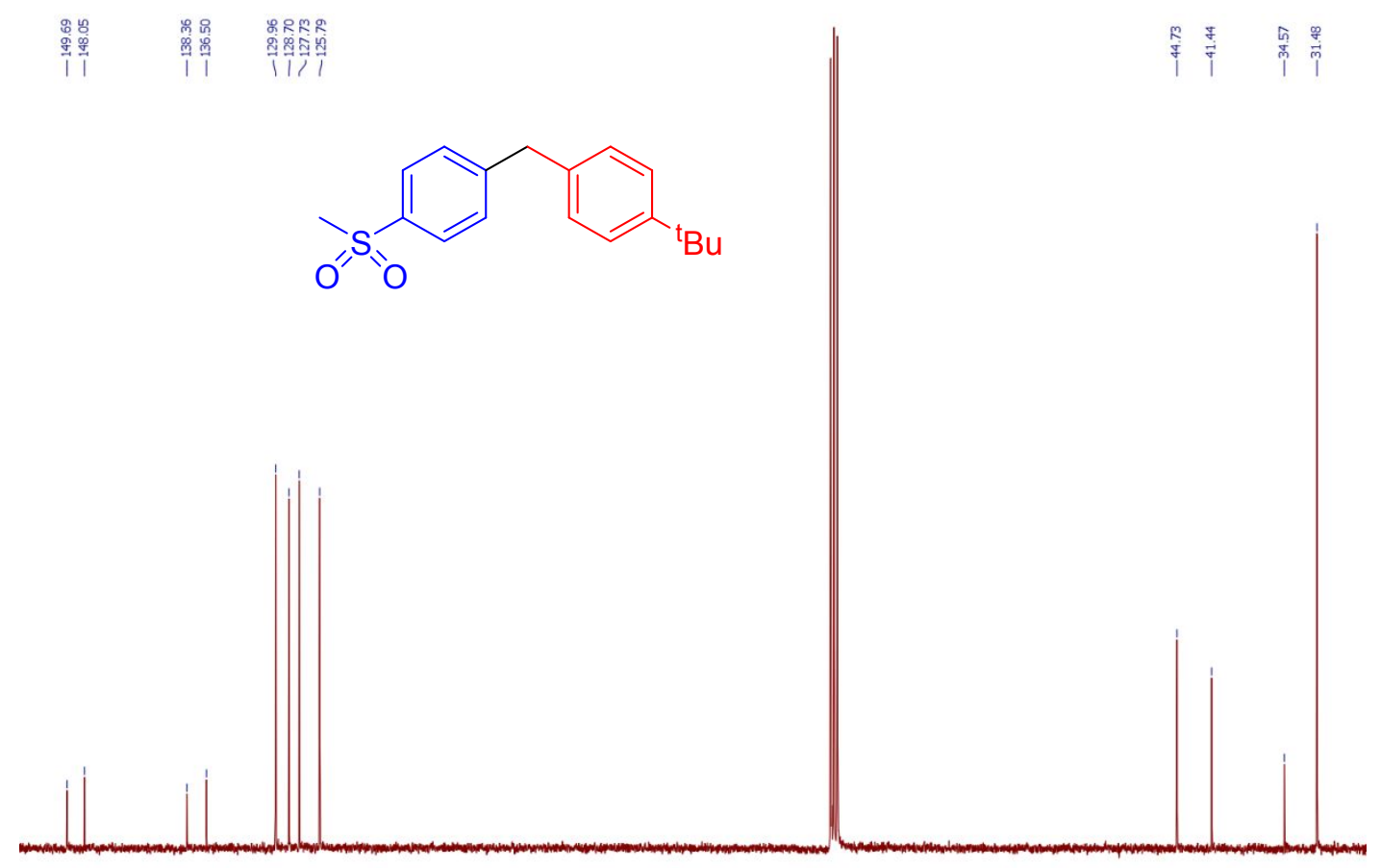

\begin{tabular}{llllllllllllllllllllllllllllllllllllll}
\hline & 150 & 145 & 140 & 1 & 135 & 130 & 125 & 120 & 115 & 110 & 105 & 100 & 95 & 90 & 85 & 80 & 75 & 70 & 65 & 60 & 55 & 50 & 45 & 40 & 35 & 30
\end{tabular}

Figure $\mathbf{S} 51 .{ }^{13} \mathrm{C}\left\{{ }^{1} \mathrm{H}\right\}$ NMR spectrum $\left(150 \mathrm{MHz}, \mathrm{CDCl}_{3}\right)$ of $\mathbf{9 j}$. 
$\mathfrak{m}_{\substack{0.0 \\ \infty}}^{\infty}$

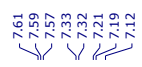

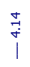

ร $\iiint$
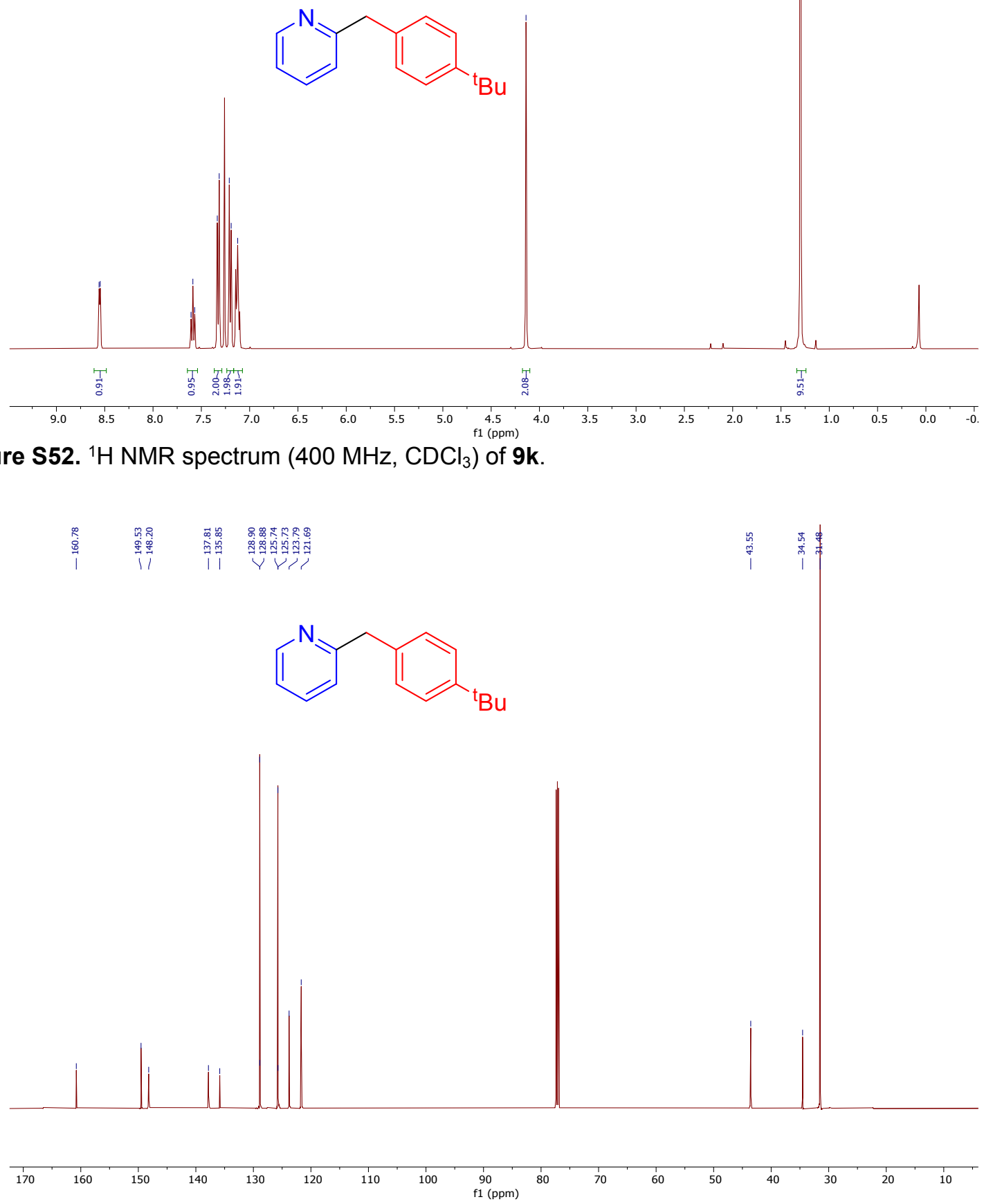

Figure S53. ${ }^{13} \mathrm{C}\left\{{ }^{1} \mathrm{H}\right\} \mathrm{NMR}$ spectrum $\left(150 \mathrm{MHz}, \mathrm{CDCl}_{3}\right)$ of $\mathbf{9 k}$.

S65 


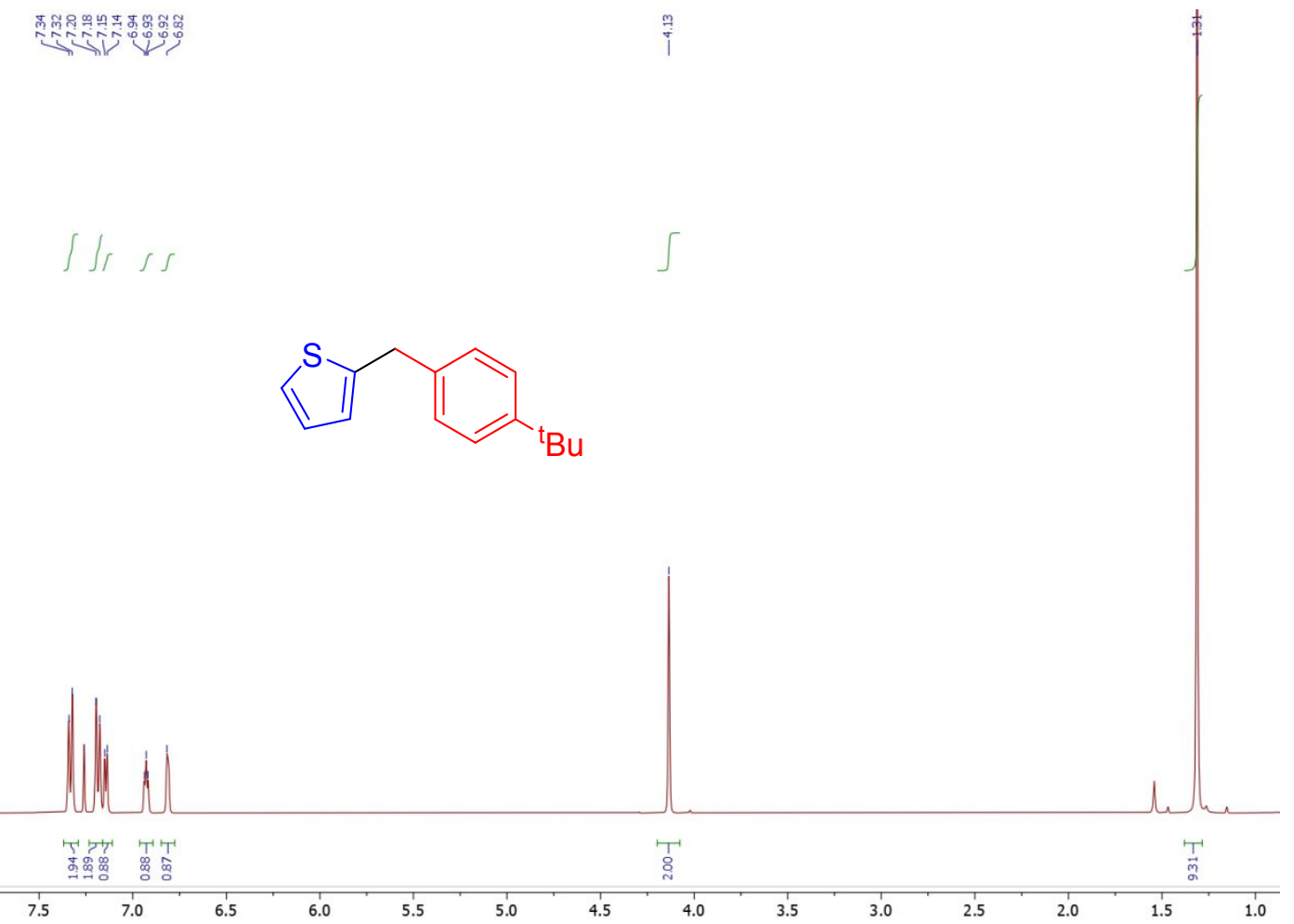

Figure S54. ${ }^{1} \mathrm{H}$ NMR spectrum $\left(400 \mathrm{MHz}, \mathrm{CDCl}_{3}\right)$ of 91 . 

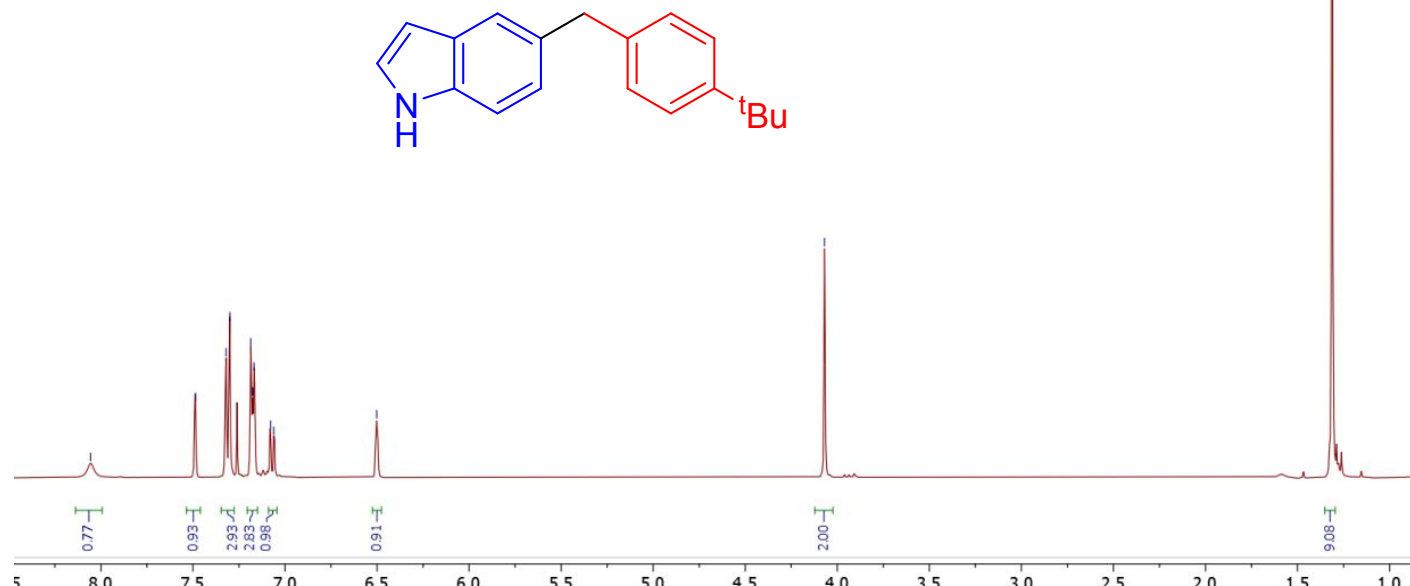

Figure S55. ${ }^{1} \mathrm{H}$ NMR spectrum $\left(400 \mathrm{MHz}, \mathrm{CDCl}_{3}\right)$ of $\mathbf{9 m}$.

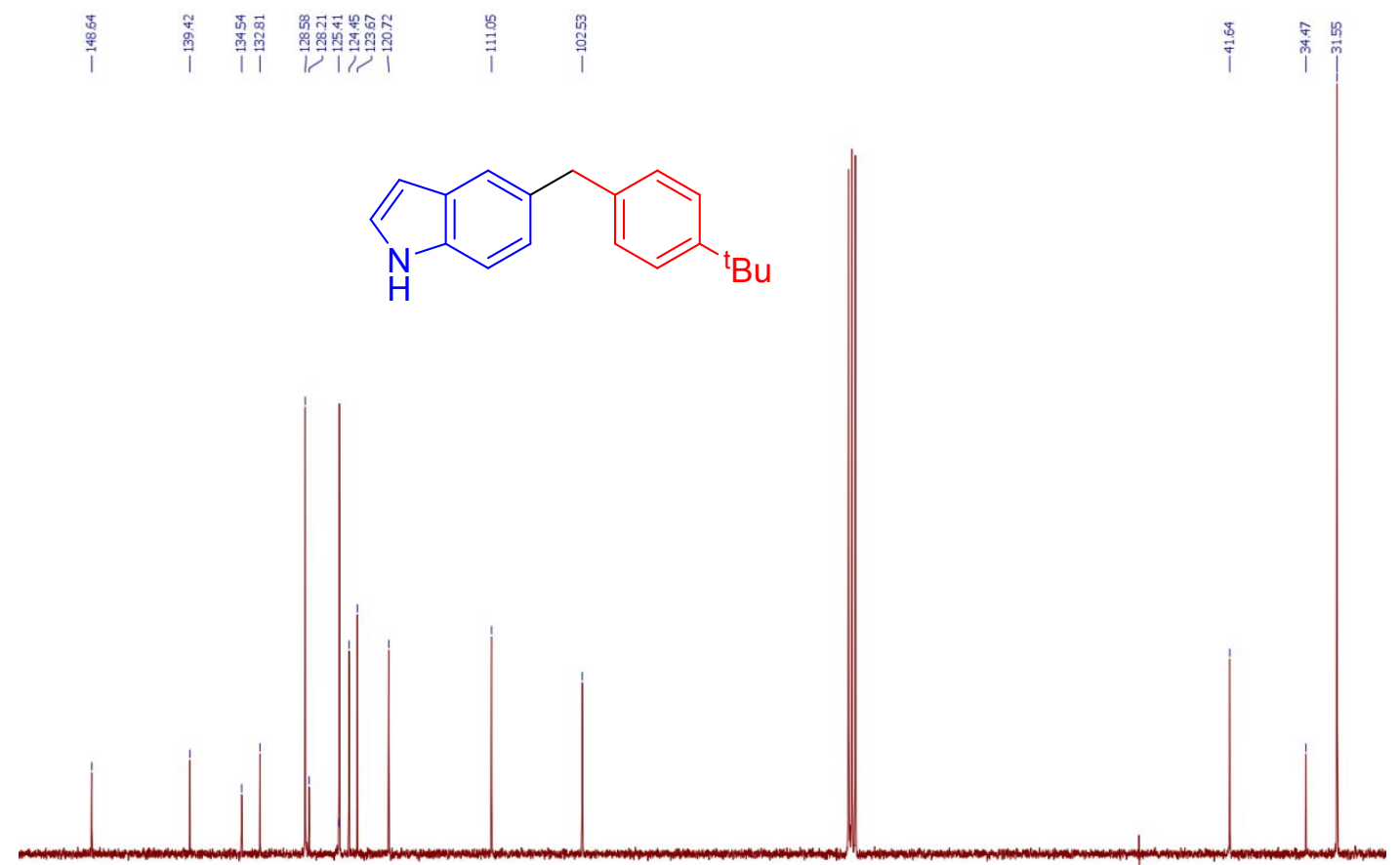

\begin{tabular}{llllllllllllllllllllllllllllllll}
\hline 55 & 150 & 145 & 140 & 135 & 130 & 125 & 120 & 115 & 110 & 105 & 100 & 95 & 90 & 85 & 80 & 75 & 70 & 65 & 60 & 55 & 50 & 45 & 40 & 35 & 30
\end{tabular} Figure S56. ${ }^{13} \mathrm{C}\left\{{ }^{1} \mathrm{H}\right\}$ NMR spectrum $\left(150 \mathrm{MHz}, \mathrm{CDCl}_{3}\right)$ of $\mathbf{9 m}$. 


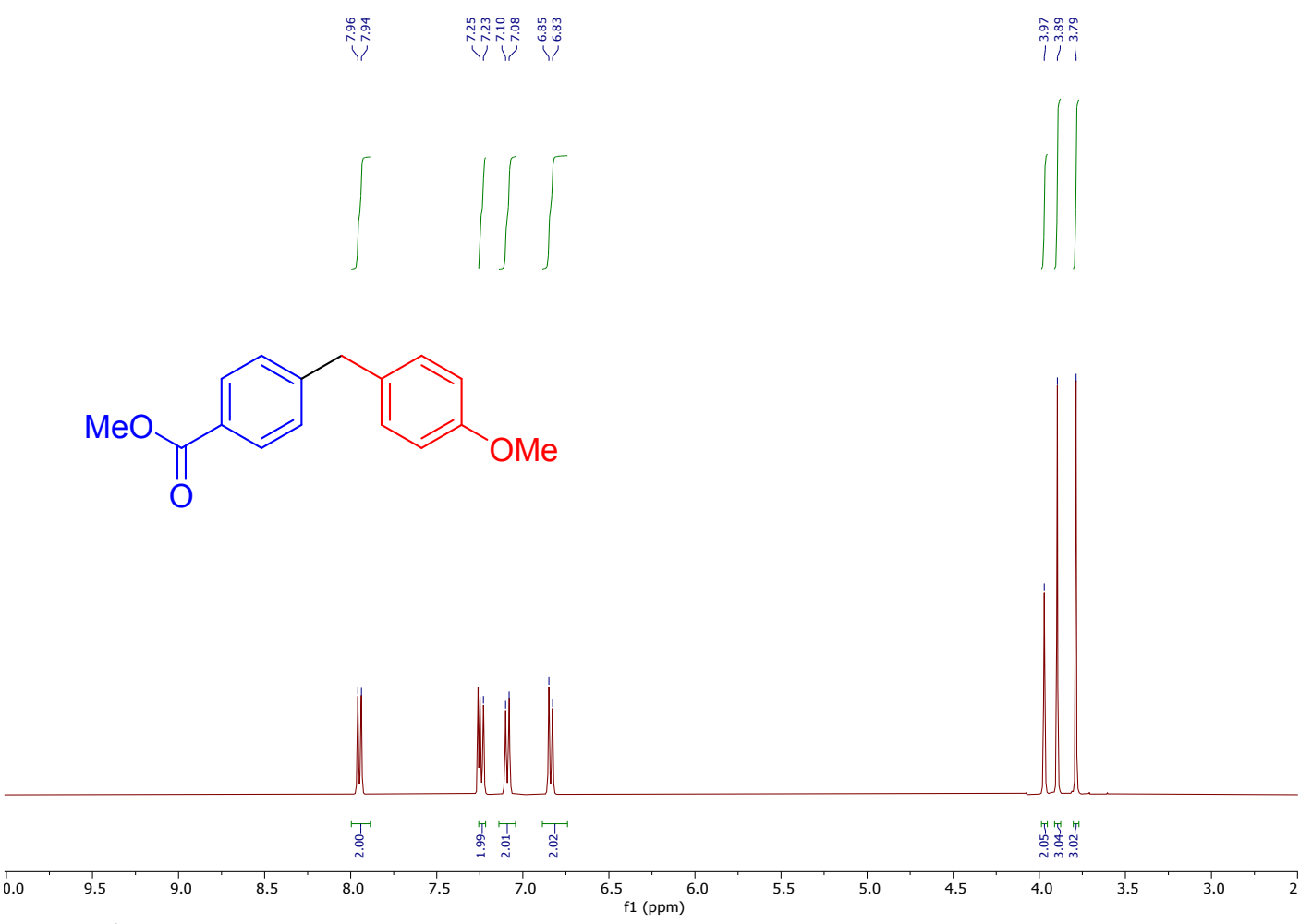

Figure S57. ${ }^{1} \mathrm{H}$ NMR spectrum $\left(400 \mathrm{MHz}, \mathrm{CDCl}_{3}\right)$ of $9 \mathbf{n}$.

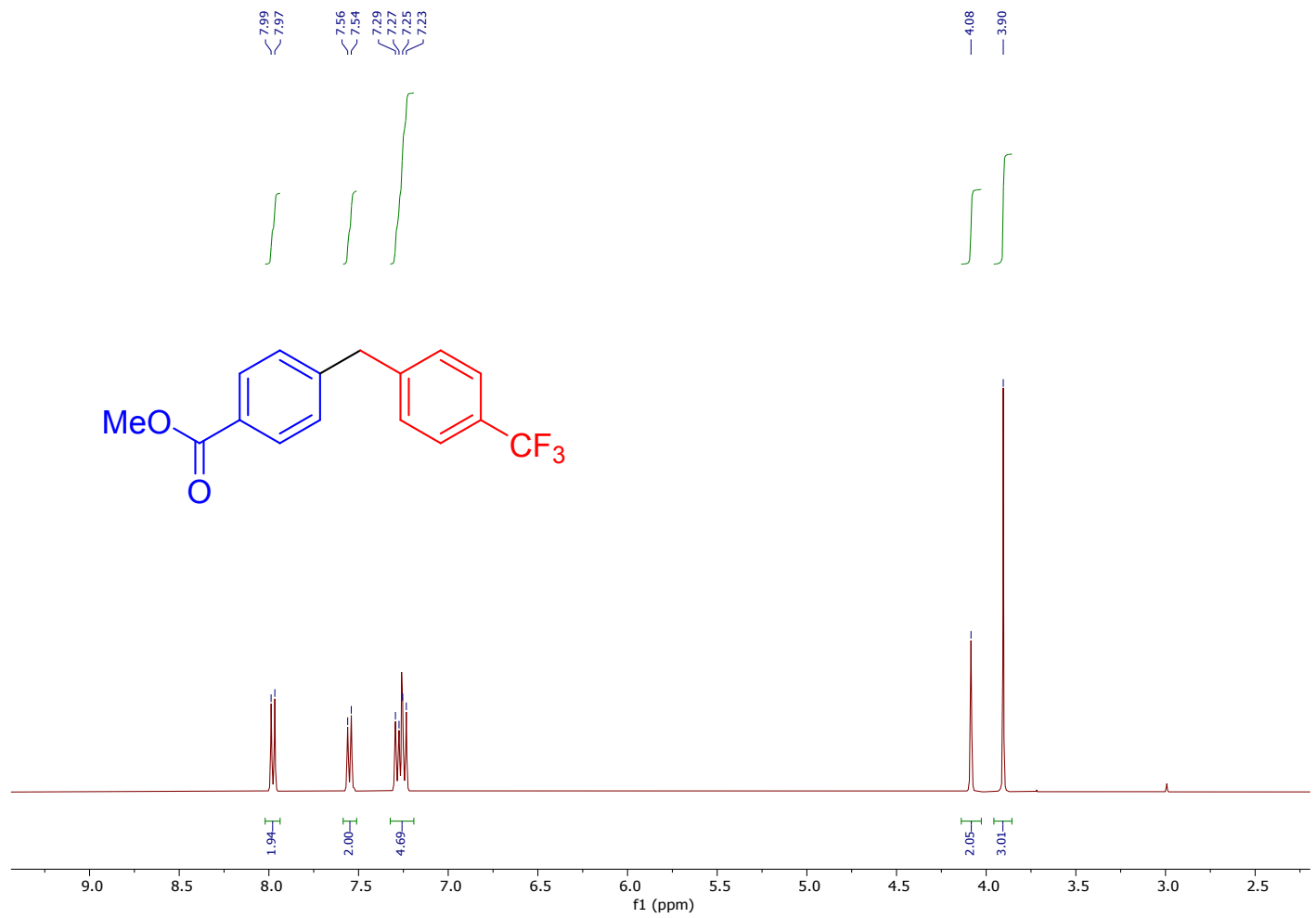

Figure S58. ${ }^{1} \mathrm{H}$ NMR spectrum $\left(400 \mathrm{MHz}, \mathrm{CDCl}_{3}\right)$ of $\mathbf{9 o}$. 


\section{SXX. Cyclic Voltammetry}

Cyclic voltammograms were recorded in an M-Braun glovebox under an $\mathrm{N}_{2}$ atmosphere in DMF using concentrations of $0.1 \mathrm{mM}$ tetraaminoethylene and $0.1 \mathrm{mM}$ tetrabutylammonium hexafluorophosphate at a scan rate of $100 \mathrm{mVs}^{-1}$. Reduction potentials were calibrated against a ferrocene $(\mathrm{Fc})$ internal standard and are reported versus $\mathrm{Fc} / \mathrm{Fc}^{+}$.

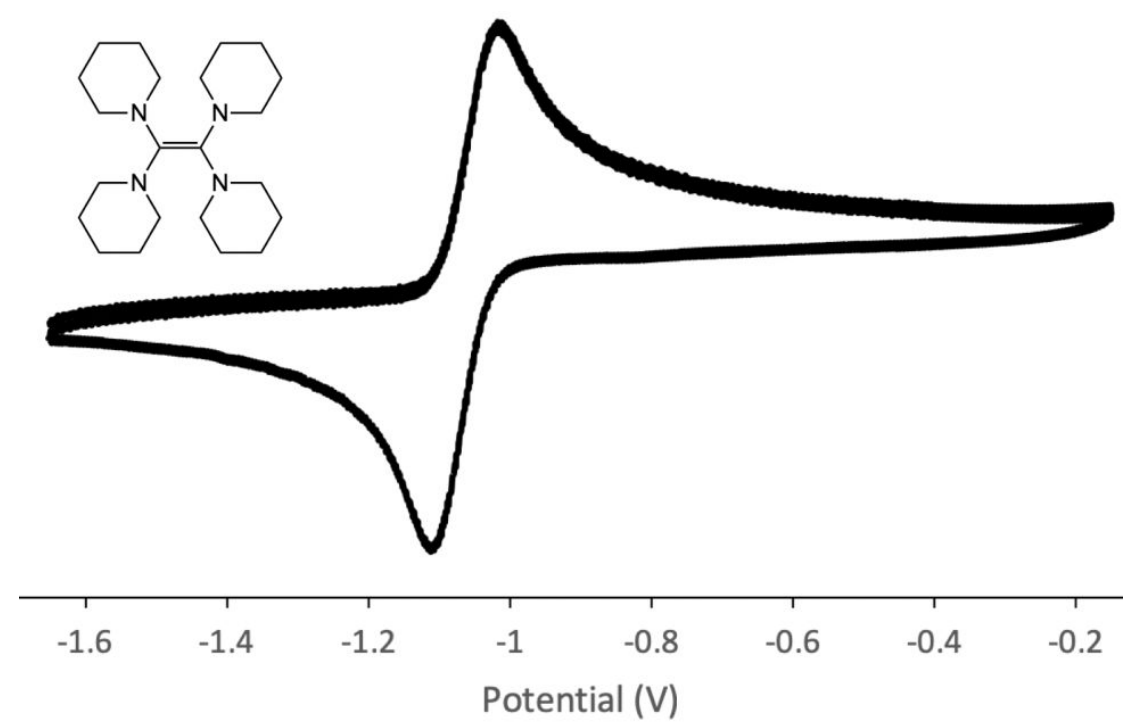

Figure S59. Cyclic voltammogram of TPiE.

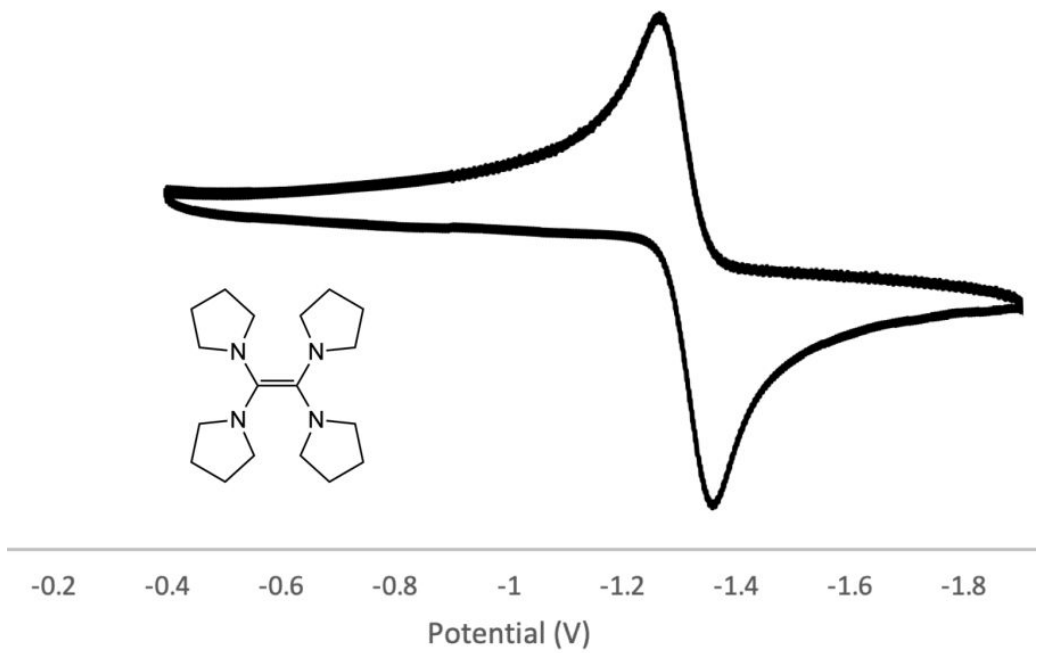

Figure S60. Cyclic voltammogram of TPyE. 


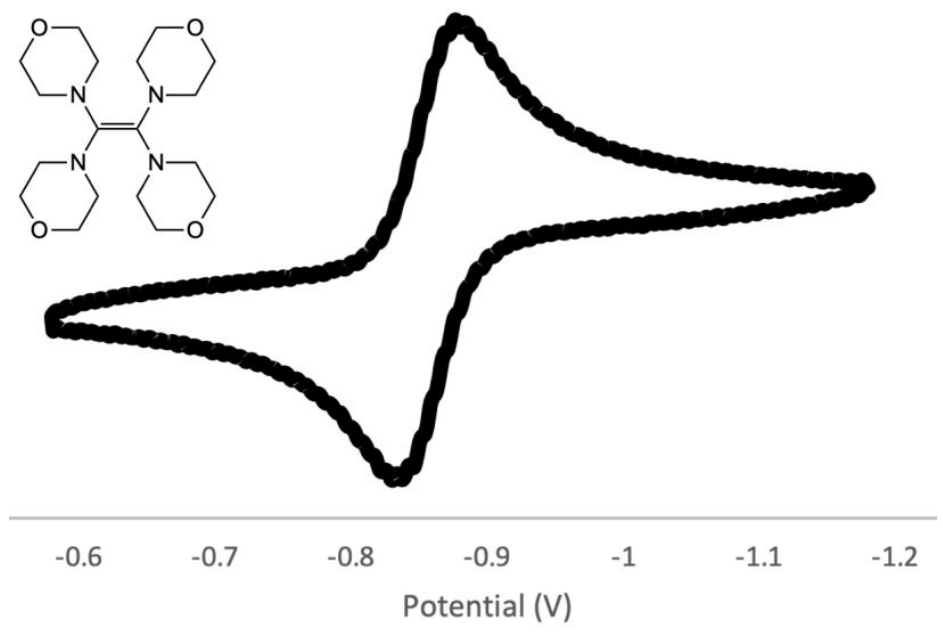

Figure S61. Cyclic voltammogram of TME.

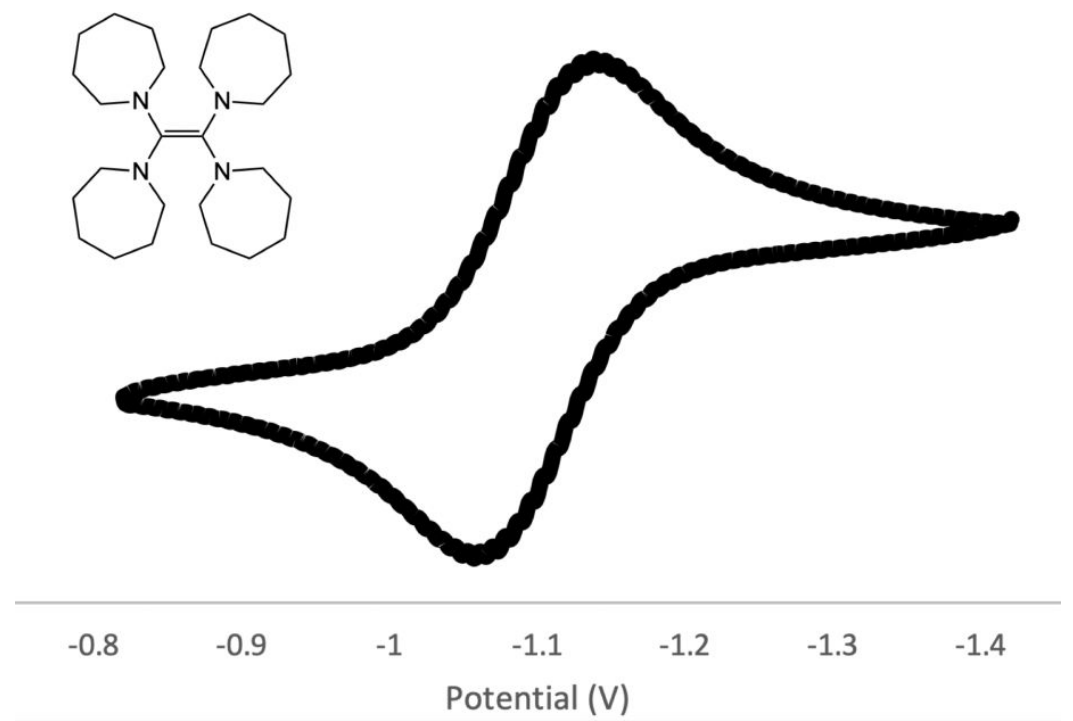

Figure S62. Cyclic voltammogram of TAzE. 


\section{SXXI. X-Ray Crystallographic Information}

$X$-Ray Diffraction of TPiE: Crystals suitable for X-ray diffraction were grown by heating a supersaturated mixture of TPiE in DMAc at $80^{\circ} \mathrm{C}$. Once all TPiE had dissolved, the solution was allowed to cool slowly to room temperature, resulting in colorless crystals. Low-temperature diffraction data ( $\omega$-scans) were collected on a Rigaku MicroMax-007HF diffractometer coupled to a Dectris Pilatus3R detector with Mo K $\alpha(\lambda=0.71073 \AA)$ for the structure of TPiE. The diffraction images were processed and scaled using Rigaku Oxford Diffraction software. ${ }^{21}$ The structure was solved with SHELXT and was refined against $\mathrm{F}^{2}$ on all data by full-matrix least squares with SHELXL. ${ }^{22}$ All non-hydrogen atoms were refined anisotropically. Hydrogen atoms were included in the model at geometrically calculated positions and refined using a riding model. The isotropic displacement parameters of all hydrogen atoms were fixed to 1.2 times the $U$ value of the atoms to which they are linked (1.5 times for methyl groups). Both piperidine models are disordered over two positions. Their site occupancies were freely refined and converged to nearly equal population distributions ( 50/50). All chemically equivalent C-C and C-N distances were restrained to be similar. The full numbering scheme of TPiE can be found in the full details of the X-ray structure determination (CIF), which is included as Supporting Information. CCDC number 2111617 contains the supplementary crystallographic data for this paper.

Table S7. Crystal data and structure refinement for TPiE.

$\begin{array}{lll}\text { Empirical formula } & \mathrm{C} 22 \mathrm{H} 40 \mathrm{~N} 4 \\ \text { Formula weight } & 360.58 & 93(2) \mathrm{K} \\ \text { Temperature } & 0.71073 \AA \\ \text { Wavelength } & \text { Monoclinic } & \\ \text { Crystal system } & \mathrm{P} 2 / \mathrm{n} & \alpha=90^{\circ} \\ \text { Space group } & \mathrm{a}=12.3100(3) \AA & \beta=92.468(3)^{\circ} \\ \text { Unit cell dimensions } & \mathrm{b}=6.2310(2) \AA & \gamma=90^{\circ} \\ & \mathrm{c}=14.1848(4) \AA & \\ & 1087.02(5) \AA^{3} & \\ \text { Volume } & 2 & \\ \text { Z } & 1.102 \mathrm{Mg} / \mathrm{m}^{3} & \\ \text { Density (calculated) } & 0.066 \mathrm{~mm}^{-1} & \\ \text { Absorption coefficient } & 400 & \\ \text { F(000) } & 0.200 \times 0.200 \times 0.080 \mathrm{~mm}^{3} \\ \text { Crystal size } & \text { Colorless Plate } \\ \text { Crystal color and habit } & \text { Dectris Pilatus } 3 \mathrm{R} \\ \text { Diffractometer } & 3.269 \text { to } 27.481^{\circ} . \\ \text { Theta range for data collection } & -15<=\mathrm{h}<=15,-8<=\mathrm{k}<=8,-18<=1<=18 \\ \text { Index ranges } & 21240 \\ \text { Reflections collected } & 2490[\mathrm{R}(\text { int })=0.0325] \\ \text { Independent reflections } & 2110 \\ \text { Observed reflections }(\mathrm{I}>2 \text { sigma(I) }) & 99.8 \% \\ \text { Completeness to theta }=25.242^{\circ} & \end{array}$


Absorption correction

Max. and min. transmission

Solution method

Refinement method

Data / restraints / parameters

Goodness-of-fit on $\mathrm{F}^{2}$

Final $R$ indices [I $>2 \operatorname{sigma}(\mathrm{I})]$

$\mathrm{R}$ indices (all data)

Largest diff. peak and hole
Semi-empirical from equivalents

1.00000 and 0.38634

SHELXT-2014/5 (Sheldrick, 2014)

SHELXL-2014/7 (Sheldrick, 2014)

$2490 / 40 / 210$

1.007

$\mathrm{R} 1=0.0414, \mathrm{wR} 2=0.1017$

$\mathrm{R} 1=0.0505, \mathrm{wR} 2=0.1080$

0.215 and -0.148 e. $\AA^{-3}$

Table S8. Atomic coordinates $\left(x 10^{4}\right)$ and equivalent isotropic displacement parameters $\left(\AA^{2} \times 10^{3}\right)$ for TPiE. $\mathrm{U}(\mathrm{eq})$ is defined as one third of the trace of the orthogonalized $\mathrm{U}^{\mathrm{ij}}$ tensor.

\begin{tabular}{|c|c|c|c|c|}
\hline & $\mathrm{x}$ & $\mathrm{y}$ & $\mathrm{z}$ & $\mathrm{U}(\mathrm{eq})$ \\
\hline $\mathrm{N}(1)$ & $3979(1)$ & $6816(1)$ & $2337(1)$ & $25(1)$ \\
\hline $\mathrm{N}(2)$ & $3044(1)$ & $7722(1)$ & $3734(1)$ & $26(1)$ \\
\hline $\mathrm{C}(1)$ & $2978(1)$ & $7269(1)$ & $2755(1)$ & $22(1)$ \\
\hline $\mathrm{C}(2)$ & $4025(2)$ & $5162(3)$ & $1573(2)$ & $32(1)$ \\
\hline$C(3)$ & $5153(9)$ & $4165(17)$ & $1559(7)$ & $52(2)$ \\
\hline$C(4)$ & $5967(5)$ & $5833(14)$ & $1456(4)$ & $49(1)$ \\
\hline$C(5)$ & $5884(8)$ & $7574(15)$ & $2202(6)$ & $57(2)$ \\
\hline $\mathrm{C}(6)$ & $4773(2)$ & $8477(4)$ & $2240(2)$ & $32(1)$ \\
\hline$C(7)$ & $2300(2)$ & $9383(3)$ & $4121(1)$ & $32(1)$ \\
\hline $\mathrm{C}(8)$ & $2811(5)$ & 10381(8) & $5014(4)$ & $45(1)$ \\
\hline $\mathrm{C}(9)$ & $3091(5)$ & $8628(10)$ & $5744(5)$ & $45(1)$ \\
\hline$C(10)$ & $3796(5)$ & $6952(11)$ & $5331(4)$ & $49(1)$ \\
\hline $\mathrm{C}(11)$ & $3301(2)$ & $6072(3)$ & $4401(1)$ & $30(1)$ \\
\hline $\mathrm{C}(2 \mathrm{~B})$ & $4204(2)$ & $4657(3)$ & $2145(2)$ & $28(1)$ \\
\hline$C(3 B)$ & $5063(9)$ & $4486(15)$ & $1421(6)$ & $35(1)$ \\
\hline$C(4 B)$ & $6125(5)$ & $5769(12)$ & $1749(4)$ & $40(1)$ \\
\hline$C(5 B)$ & $5841(8)$ & $8017(13)$ & $2004(6)$ & $43(1)$ \\
\hline$C(6 B)$ & $4921(2)$ & $8092(4)$ & $2700(2)$ & $31(1)$ \\
\hline$C(7 B)$ & $2910(3)$ & $9811(4)$ & $4030(2)$ & $37(1)$ \\
\hline $\mathrm{C}(8 \mathrm{~B})$ & $2568(6)$ & $9829(11)$ & $5045(5)$ & $48(1)$ \\
\hline $\mathrm{C}(9 \mathrm{~B})$ & $3385(6)$ & $8648(16)$ & $5676(8)$ & $54(2)$ \\
\hline $\mathrm{C}(10 \mathrm{~B})$ & $3579(7)$ & $6394(13)$ & $5290(6)$ & $49(2)$ \\
\hline$C(11 B)$ & $3892(3)$ & $6466(5)$ & $4283(2)$ & $38(1)$ \\
\hline
\end{tabular}

Table S9. Bond lengths $[\AA]$ and angles $\left[{ }^{\circ}\right]$ for TPiE.

\begin{tabular}{ll}
\hline $\mathrm{N}(1)-\mathrm{C}(2 \mathrm{~B})$ & $1.403(2)$ \\
$\mathrm{N}(1)-\mathrm{C}(1)$ & $1.4177(11)$ \\
$\mathrm{N}(1)-\mathrm{C}(6)$ & $1.435(2)$ \\
$\mathrm{N}(1)-\mathrm{C}(6 \mathrm{~B})$ & $1.480(2)$ \\
$\mathrm{N}(1)-\mathrm{C}(2)$ & $1.499(2)$ \\
$\mathrm{N}(2)-\mathrm{C}(7 \mathrm{~B})$ & $1.380(3)$ \\
$\mathrm{N}(2)-\mathrm{C}(1)$ & $1.4161(12)$ \\
$\mathrm{N}(2)-\mathrm{C}(11)$ & $1.4237(19)$ \\
$\mathrm{N}(2)-\mathrm{C}(11 \mathrm{~B})$ & $1.496(3)$ \\
$\mathrm{N}(2)-\mathrm{C}(7)$ & $1.502(2)$ \\
$\mathrm{C}(1)-\mathrm{C}(1) \# 1$ & $1.3549(17)$ \\
$\mathrm{C}(2)-\mathrm{C}(3)$ & $1.522(11)$
\end{tabular}




$\begin{array}{lc}\mathrm{C}(3)-\mathrm{C}(4) & 1.455(13) \\ \mathrm{C}(4)-\mathrm{C}(5) & 1.522(12) \\ \mathrm{C}(5)-\mathrm{C}(6) & 1.482(10) \\ \mathrm{C}(7)-\mathrm{C}(8) & 1.523(5) \\ \mathrm{C}(8)-\mathrm{C}(9) & 1.534(7) \\ \mathrm{C}(9)-\mathrm{C}(10) & 1.494(8) \\ \mathrm{C}(10)-\mathrm{C}(11) & 1.530(6) \\ \mathrm{C}(2 \mathrm{~B})-\mathrm{C}(3 \mathrm{~B}) & 1.511(11) \\ \mathrm{C}(3 \mathrm{~B})-\mathrm{C}(4 \mathrm{~B}) & 1.585(11) \\ \mathrm{C}(4 \mathrm{~B})-\mathrm{C}(5 \mathrm{~B}) & 1.492(12) \\ \mathrm{C}(5 \mathrm{~B})-\mathrm{C}(6 \mathrm{~B}) & 1.535(8) \\ \mathrm{C}(7 \mathrm{~B})-\mathrm{C}(8 \mathrm{~B}) & 1.517(7) \\ \mathrm{C}(8 \mathrm{~B})-\mathrm{C}(9 \mathrm{~B}) & 1.509(10) \\ \mathrm{C}(9 \mathrm{~B})-\mathrm{C}(10 \mathrm{~B}) & 1.530(10) \\ \mathrm{C}(10 \mathrm{~B})-\mathrm{C}(11 \mathrm{~B}) & 1.495(9) \\ & \\ \mathrm{C}(2 \mathrm{~B})-\mathrm{N}(1)-\mathrm{C}(1) & 117.03(10) \\ \mathrm{C}(1)-\mathrm{N}(1)-\mathrm{C}(6) & 120.30(12) \\ \mathrm{C}(2 \mathrm{~B})-\mathrm{N}(1)-\mathrm{C}(6 \mathrm{~B}) & 115.12(15) \\ \mathrm{C}(1)-\mathrm{N}(1)-\mathrm{C}(6 \mathrm{~B}) & 115.45(11) \\ \mathrm{C}(1)-\mathrm{N}(1)-\mathrm{C}(2) & 120.01(10) \\ \mathrm{C}(6)-\mathrm{N}(1)-\mathrm{C}(2) & 112.28(16) \\ \mathrm{C}(7 \mathrm{~B})-\mathrm{N}(2)-\mathrm{C}(1) & 118.95(13) \\ \mathrm{C}(1)-\mathrm{N}(2)-\mathrm{C}(11) & 120.62(10) \\ \mathrm{C}(7 \mathrm{~B})-\mathrm{N}(2)-\mathrm{C}(11 \mathrm{~B}) & 115.2(2) \\ \mathrm{C}(1)-\mathrm{N}(2)-\mathrm{C}(11 \mathrm{~B}) & 114.57(13) \\ \mathrm{C}(1)-\mathrm{N}(2)-\mathrm{C}(7) & 119.05(10) \\ \mathrm{C}(11)-\mathrm{N}(2)-\mathrm{C}(7) & 112.11(14) \\ \mathrm{C}(1) \# 1-\mathrm{C}(1)-\mathrm{N}(2) & 122.36(10) \\ \mathrm{C}(1) \# 1-\mathrm{C}(1)-\mathrm{N}(1) & 121.85(10) \\ \mathrm{N}(2)-\mathrm{C}(1)-\mathrm{N}(1) & 115.79(7) \\ \mathrm{N}(1)-\mathrm{C}(2)-\mathrm{C}(3) & 110.7(4) \\ \mathrm{C}(4)-\mathrm{C}(3)-\mathrm{C}(2) & 110.0(7) \\ \mathrm{C}(3)-\mathrm{C}(4)-\mathrm{C}(5) & 111.9(7) \\ \mathrm{C}(6)-\mathrm{C}(5)-\mathrm{C}(4) & 112.7(7) \\ \mathrm{N}(1)-\mathrm{C}(6)-\mathrm{C}(5) & 111.3(4) \\ \mathrm{N}(2)-\mathrm{C}(7)-\mathrm{C}(8) & 110.4(3) \\ \mathrm{C}(7)-\mathrm{C}(8)-\mathrm{C}(9) & 110.1(4) \\ \mathrm{C}(10)-\mathrm{C}(9)-\mathrm{C}(8) & 110.5(6) \\ \mathrm{C}(9)-\mathrm{C}(10)-\mathrm{C}(11) & 111.9(5) \\ \mathrm{N}(2)-\mathrm{C}(11)-\mathrm{C}(10) & 112.5(3) \\ \mathrm{N}(1)-\mathrm{C}(2 \mathrm{~B})-\mathrm{C}(3 \mathrm{~B}) & 110.5(4) \\ \mathrm{C}(2 \mathrm{~B})-\mathrm{C}(3 \mathrm{~B})-\mathrm{C}(4 \mathrm{~B}) & 110.9(6) \\ \mathrm{C}(5 \mathrm{~B})-\mathrm{C}(4 \mathrm{~B})-\mathrm{C}(3 \mathrm{~B}) & 110.2(7) \\ \mathrm{C}(4 \mathrm{~B})-\mathrm{C}(5 \mathrm{~B})-\mathrm{C}(6 \mathrm{~B}) & 111.8(6) \\ \mathrm{N}(1)-\mathrm{C}(6 \mathrm{~B})-\mathrm{C}(5 \mathrm{~B}) & 110.5(4) \\ \mathrm{N}(2)-\mathrm{C}(7 \mathrm{~B})-\mathrm{C}(8 \mathrm{~B}) & 109.7(3) \\ \mathrm{C}(9 \mathrm{~B})-\mathrm{C}(8 \mathrm{~B})-\mathrm{C}(7 \mathrm{~B}) & 110.8(7) \\ \mathrm{C}(8 \mathrm{~B})-\mathrm{C}(9 \mathrm{~B})-\mathrm{C}(10 \mathrm{~B}) & 110.2(8) \\ \mathrm{C}(11 \mathrm{~B})-\mathrm{C}(10 \mathrm{~B})-\mathrm{C}(9 \mathrm{~B}) & 111.4(7) \\ \mathrm{N}(2)-\mathrm{C}(11 \mathrm{~B})-\mathrm{C}(10 \mathrm{~B}) & 108.1(4) \\ & \end{array}$

Symmetry transformations used to generate equivalent atoms:

\#1 - $\mathrm{x}+1 / 2, \mathrm{y},-\mathrm{z}+1 / 2$ 
Table S10. Anisotropic displacement parameters $\left(\AA^{2} \times 10^{3}\right)$ for TPiE. The anisotropic displacement factor exponent takes the form: $-2 \pi^{2}\left[h^{2} a^{* 2} U^{11}+\ldots+2 h k a^{*} b^{*} U^{12}\right]$

\begin{tabular}{lcccccc}
\hline & $\mathrm{U}^{11}$ & $\mathrm{U}^{22}$ & $\mathrm{U}^{33}$ & $\mathrm{U}^{23}$ & $\mathrm{U}^{13}$ & $\mathrm{U}^{12}$ \\
\hline $\mathrm{N}(1)$ & $21(1)$ & $24(1)$ & $29(1)$ & $-3(1)$ & $2(1)$ & $-1(1)$ \\
$\mathrm{N}(2)$ & $24(1)$ & $28(1)$ & $25(1)$ & $-2(1)$ & $1(1)$ & $0(1)$ \\
$\mathrm{C}(1)$ & $24(1)$ & $17(1)$ & $27(1)$ & $1(1)$ & $3(1)$ & $-1(1)$ \\
$\mathrm{C}(2)$ & $34(1)$ & $24(1)$ & $38(2)$ & $-8(1)$ & $9(1)$ & $-2(1)$ \\
$\mathrm{C}(3)$ & $41(2)$ & $40(4)$ & $77(5)$ & $-6(2)$ & $26(3)$ & $12(2)$ \\
$\mathrm{C}(4)$ & $22(2)$ & $69(3)$ & $57(3)$ & $-27(3)$ & $1(2)$ & $8(2)$ \\
$\mathrm{C}(5)$ & $32(2)$ & $71(5)$ & $68(3)$ & $-12(3)$ & $17(2)$ & $-23(3)$ \\
$\mathrm{C}(6)$ & $32(1)$ & $31(1)$ & $35(1)$ & $-5(1)$ & $4(1)$ & $-10(1)$ \\
$\mathrm{C}(7)$ & $42(1)$ & $23(1)$ & $33(1)$ & $-5(1)$ & $10(1)$ & $4(1)$ \\
$\mathrm{C}(8)$ & $62(2)$ & $34(2)$ & $40(1)$ & $-17(1)$ & $17(1)$ & $-17(1)$ \\
$\mathrm{C}(9)$ & $48(3)$ & $61(2)$ & $26(2)$ & $-12(1)$ & $9(2)$ & $-20(2)$ \\
$\mathrm{C}(10)$ & $48(2)$ & $74(3)$ & $24(1)$ & $-3(2)$ & $-8(1)$ & $-4(2)$ \\
$\mathrm{C}(11)$ & $32(1)$ & $33(1)$ & $26(1)$ & $2(1)$ & $1(1)$ & $2(1)$ \\
$\mathrm{C}(2 \mathrm{~B})$ & $28(1)$ & $24(1)$ & $31(1)$ & $-1(1)$ & $4(1)$ & $3(1)$ \\
$\mathrm{C}(3 \mathrm{~B})$ & $44(3)$ & $26(2)$ & $36(2)$ & $-7(2)$ & $10(1)$ & $6(2)$ \\
$\mathrm{C}(4 \mathrm{~B})$ & $22(2)$ & $51(2)$ & $49(3)$ & $-8(2)$ & $6(2)$ & $-1(2)$ \\
$\mathrm{C}(5 \mathrm{~B})$ & $37(2)$ & $33(2)$ & $62(3)$ & $-14(2)$ & $29(2)$ & $-12(2)$ \\
$\mathrm{C}(6 \mathrm{~B})$ & $25(1)$ & $35(1)$ & $34(1)$ & $-9(1)$ & $4(1)$ & $-7(1)$ \\
$\mathrm{C}(7 \mathrm{~B})$ & $44(2)$ & $28(1)$ & $38(1)$ & $-6(1)$ & $10(1)$ & $-7(1)$ \\
$\mathrm{C}(8 \mathrm{~B})$ & $61(3)$ & $39(3)$ & $46(2)$ & $-19(2)$ & $18(2)$ & $-11(2)$ \\
$\mathrm{C}(9 \mathrm{~B})$ & $52(4)$ & $83(4)$ & $27(2)$ & $-11(2)$ & $6(3)$ & $-29(3)$ \\
$\mathrm{C}(10 \mathrm{~B})$ & $48(3)$ & $63(4)$ & $35(2)$ & $1(2)$ & $-5(2)$ & $14(3)$ \\
$\mathrm{C}(11 \mathrm{~B})$ & $26(2)$ & $52(2)$ & $37(1)$ & $4(1)$ & $0(1)$ & $4(1)$ \\
& & & & & & \\
\hline
\end{tabular}

Table S11. Torsion angles $\left[{ }^{\circ}\right]$ for TPiE.

\begin{tabular}{lc}
\hline C(7B)-N(2)-C(1)-C(1)\#1 & $-73.5(2)$ \\
C(11)-N(2)-C(1)-C(1)\#1 & $109.71(14)$ \\
C(11B)-N(2)-C(1)-C(1)\#1 & $144.56(17)$ \\
C(7)-N(2)-C(1)-C(1)\#1 & $-36.30(15)$ \\
C(7B)-N(2)-C(1)-N(1) & $106.4(2)$ \\
C(11)-N(2)-C(1)-N(1) & $-70.39(16)$ \\
C(11B)-N(2)-C(1)-N(1) & $-35.5(2)$ \\
C(7)-N(2)-C(1)-N(1) & $143.60(14)$ \\
C(2B)-N(1)-C(1)-C(1)\#1 & $-77.09(15)$ \\
C(6)-N(1)-C(1)-C(1)\#1 & $111.02(17)$ \\
C(6B)-N(1)-C(1)-C(1)\#1 & $142.50(16)$ \\
C(2)-N(1)-C(1)-C(1)\#1 & $-36.62(16)$ \\
C(2B)-N(1)-C(1)-N(2) & $103.01(15)$ \\
C(6)-N(1)-C(1)-N(2) & $-68.88(19)$ \\
C(6B)-N(1)-C(1)-N(2) & $-37.40(18)$ \\
C(2)-N(1)-C(1)-N(2) & $143.48(15)$ \\
C(1)-N(1)-C(2)-C(3) & $-152.6(4)$ \\
C(6)-N(1)-C(2)-C(3) & $57.3(5)$ \\
N(1)-C(2)-C(3)-C(4) & $-56.0(7)$ \\
C(2)-C(3)-C(4)-C(5) & $53.7(9)$ \\
C(3)-C(4)-C(5)-C(6) & $-52.9(9)$ \\
C(1)-N(1)-C(6)-C(5) & $154.7(4)$ \\
C(2)-N(1)-C(6)-C(5) & $-55.4(5)$
\end{tabular}




$\begin{array}{lc}\mathrm{C}(4)-\mathrm{C}(5)-\mathrm{C}(6)-\mathrm{N}(1) & 52.6(7) \\ \mathrm{C}(1)-\mathrm{N}(2)-\mathrm{C}(7)-\mathrm{C}(8) & -153.4(2) \\ \mathrm{C}(11)-\mathrm{N}(2)-\mathrm{C}(7)-\mathrm{C}(8) & 57.9(3) \\ \mathrm{N}(2)-\mathrm{C}(7)-\mathrm{C}(8)-\mathrm{C}(9) & -56.5(5) \\ \mathrm{C}(7)-\mathrm{C}(8)-\mathrm{C}(9)-\mathrm{C}(10) & 54.9(6) \\ \mathrm{C}(8)-\mathrm{C}(9)-\mathrm{C}(10)-\mathrm{C}(11) & -52.7(6) \\ \mathrm{C}(1)-\mathrm{N}(2)-\mathrm{C}(11)-\mathrm{C}(10) & 155.9(3) \\ \mathrm{C}(7)-\mathrm{N}(2)-\mathrm{C}(11)-\mathrm{C}(10) & -56.0(4) \\ \mathrm{C}(9)-\mathrm{C}(10)-\mathrm{C}(11)-\mathrm{N}(2) & 54.1(6) \\ \mathrm{C}(1)-\mathrm{N}(1)-\mathrm{C}(2 \mathrm{~B})-\mathrm{C}(3 \mathrm{~B}) & 160.4(4) \\ \mathrm{C}(6 \mathrm{~B})-\mathrm{N}(1)-\mathrm{C}(2 \mathrm{~B})-\mathrm{C}(3 \mathrm{~B}) & -59.1(5) \\ \mathrm{N}(1)-\mathrm{C}(2 \mathrm{~B})-\mathrm{C}(3 \mathrm{~B})-\mathrm{C}(4 \mathrm{~B}) & 55.3(7) \\ \mathrm{C}(2 \mathrm{~B})-\mathrm{C}(3 \mathrm{~B})-\mathrm{C}(4 \mathrm{~B})-\mathrm{C}(5 \mathrm{~B}) & -52.6(8) \\ \mathrm{C}(3 \mathrm{~B})-\mathrm{C}(4 \mathrm{~B})-\mathrm{C}(5 \mathrm{~B})-\mathrm{C}(6 \mathrm{~B}) & 51.1(9) \\ \mathrm{C}(2 \mathrm{~B})-\mathrm{N}(1)-\mathrm{C}(6 \mathrm{~B})-\mathrm{C}(5 \mathrm{~B}) & 57.2(5) \\ \mathrm{C}(1)-\mathrm{N}(1)-\mathrm{C}(6 \mathrm{~B})-\mathrm{C}(5 \mathrm{~B}) & -161.7(4) \\ \mathrm{C}(4 \mathrm{~B})-\mathrm{C}(5 \mathrm{~B})-\mathrm{C}(6 \mathrm{~B})-\mathrm{N}(1) & -52.4(7) \\ \mathrm{C}(1)-\mathrm{N}(2)-\mathrm{C}(7 \mathrm{~B})-\mathrm{C}(8 \mathrm{~B}) & 157.7(3) \\ \mathrm{C}(11 \mathrm{~B})-\mathrm{N}(2)-\mathrm{C}(7 \mathrm{~B})-\mathrm{C}(8 \mathrm{~B}) & -60.6(5) \\ \mathrm{N}(2)-\mathrm{C}(7 \mathrm{~B})-\mathrm{C}(8 \mathrm{~B})-\mathrm{C}(9 \mathrm{~B}) & 56.6(6) \\ \mathrm{C}(7 \mathrm{~B})-\mathrm{C}(8 \mathrm{~B})-\mathrm{C}(9 \mathrm{~B})-\mathrm{C}(10 \mathrm{~B}) & -53.4(8) \\ \text { C(8B)-C(9B)-C(10B)-C(11B) } & 53.9(9) \\ \text { C(7B)-N(2)-C(11B)-C(10B) } & 59.8(5) \\ \text { C(1)-N(2)-C(11B)-C(10B) } & -156.8(4) \\ \text { C(9B)-C(10B)-C(11B)-N(2) } & -53.8(7)\end{array}$

Symmetry transformations used to generate equivalent atoms: $\# 1-\mathrm{x}+1 / 2, \mathrm{y},-\mathrm{z}+1 / 2$ 
$X$-Ray Diffraction of TPyE: Crystals suitable for X-ray diffraction were grown by heating a supersaturated mixture of TPyE in DMAc at $80^{\circ} \mathrm{C}$. Once all TPyE had dissolved, the solution was allowed to cool slowly to room temperature, resulting in colorless crystals. Low-temperature diffraction data ( $\omega$-scans) were collected on a Rigaku MicroMax-007HF diffractometer coupled to a Dectris Pilatus3R detector with Mo K $\alpha(\lambda=0.71073 \AA)$ for the structure of TPyE. The diffraction images were processed and scaled using Rigaku Oxford Diffraction software. ${ }^{21}$ The structure was solved with SHELXT and was refined against $\mathrm{F}^{2}$ on all data by full-matrix least squares with SHELXL. ${ }^{22}$ All non-hydrogen atoms were refined anisotropically. Hydrogen atoms were included in the model at geometrically calculated positions and refined using a riding model. The isotropic displacement parameters of all hydrogen atoms were fixed to 1.2 times the $\mathrm{U}$ value of the atoms to which they are linked (1.5 times for methyl groups). The full numbering scheme can be found in the full details of the X-ray structure determination (CIF), which is included as Supporting Information. CCDC number 2111616 contains the supplementary crystallographic data for this paper.

Table S12. Crystal data and structure refinement for TPyE.

Empirical formula

Formula weight

Temperature

Wavelength

Crystal system

Space group

Unit cell dimensions

Volume

Z

Density (calculated)

Absorption coefficient

$\mathrm{F}(000)$

Crystal size

Crystal color and habit

Diffractometer

Theta range for data collection

Index ranges

Reflections collected

Independent reflections

Observed reflections (I $>2$ sigma(I))

Completeness to theta $=25.242^{\circ}$

Absorption correction

Max. and min. transmission

Solution method

Refinement method

Data / restraints / parameters

Goodness-of-fit on $\mathrm{F}^{2}$

Final R indices [I $>2 \operatorname{sigma}(\mathrm{I})]$
C18 H32 N4

304.47

93(2) K

$0.71073 \AA$

Triclinic

P-1

$\mathrm{a}=5.9752(2) \AA$

$\alpha=92.009(3)^{\circ}$

$\mathrm{b}=11.9721(4) \AA$

$\beta=96.897(3)^{\circ}$

$\mathrm{c}=12.2482(5) \AA$

$866.15(5) \AA^{3}$

2

$1.167 \mathrm{Mg} / \mathrm{m}^{3}$

$0.071 \mathrm{~mm}^{-1}$

336

$0.350 \times 0.240 \times 0.120 \mathrm{~mm}^{3}$

Colorless Block

Dectris Pilatus 3R

3.354 to $28.279^{\circ}$.

$-7<=\mathrm{h}<=7,-15<=\mathrm{k}<=15,-16<=1<=16$

17242

$4276[\mathrm{R}(\mathrm{int})=0.0344]$

3512

$99.9 \%$

Semi-empirical from equivalents

1.00000 and 0.51122

SHELXT-2014/5 (Sheldrick, 2014)

SHELXL-2014/7 (Sheldrick, 2014)

4276 / 0 / 199

1.029

$\mathrm{R} 1=0.0491, w \mathrm{R} 2=0.1249$ 
$\mathrm{R}$ indices (all data)

Extinction coefficient

Largest diff. peak and hole
$\mathrm{R} 1=0.0606, \mathrm{wR} 2=0.1327$

$\mathrm{n} / \mathrm{a}$

0.381 and -0.204 e. $\AA^{-3}$

Table S13. Atomic coordinates $\left(\times 10^{4}\right)$ and equivalent isotropic displacement parameters $\left(\AA^{2} \times 10^{3}\right)$ for TPyE. $U(e q)$ is defined as one third of the trace of the orthogonalized $U^{i j}$ tensor.

\begin{tabular}{lrrrr}
\hline & $\mathrm{x}$ & $\mathrm{y}$ & $\mathrm{z}$ & $\mathrm{U}(\mathrm{eq})$ \\
\hline $\mathrm{N}(1)$ & $1723(2)$ & $7738(1)$ & $3889(1)$ & $24(1)$ \\
$\mathrm{N}(2)$ & $2022(2)$ & $5998(1)$ & $2945(1)$ & $26(1)$ \\
$\mathrm{N}(3)$ & $1725(2)$ & $8798(1)$ & $1770(1)$ & $22(1)$ \\
$\mathrm{N}(4)$ & $3457(2)$ & $7256(1)$ & $1146(1)$ & $31(1)$ \\
$\mathrm{C}(1)$ & $2053(2)$ & $7171(1)$ & $2907(1)$ & $20(1)$ \\
$\mathrm{C}(2)$ & $2393(2)$ & $7722(1)$ & $1985(1)$ & $21(1)$ \\
$\mathrm{C}(3)$ & $-147(2)$ & $7412(1)$ & $4494(1)$ & $23(1)$ \\
$\mathrm{C}(4)$ & $111(2)$ & $8293(1)$ & $5443(1)$ & $26(1)$ \\
$\mathrm{C}(5)$ & $2660(2)$ & $8607(1)$ & $5634(1)$ & $34(1)$ \\
$\mathrm{C}(6)$ & $3328(2)$ & $8585(1)$ & $4468(1)$ & $29(1)$ \\
$\mathrm{C}(7)$ & $3444(2)$ & $5457(1)$ & $3784(1)$ & $23(1)$ \\
$\mathrm{C}(8)$ & $3222(2)$ & $4233(1)$ & $3368(1)$ & $25(1)$ \\
$\mathrm{C}(9)$ & $885(2)$ & $4089(1)$ & $2695(1)$ & $30(1)$ \\
$\mathrm{C}(10)$ & $675(2)$ & $5217(1)$ & $2162(1)$ & $24(1)$ \\
$\mathrm{C}(11)$ & $-260(2)$ & $9224(1)$ & $2162(1)$ & $22(1)$ \\
$\mathrm{C}(12)$ & $-324(2)$ & $10353(1)$ & $1641(1)$ & $26(1)$ \\
$\mathrm{C}(13)$ & $2170(3)$ & $10782(1)$ & $1740(2)$ & $37(1)$ \\
$\mathrm{C}(14)$ & $3441(2)$ & $9724(1)$ & $1675(1)$ & $24(1)$ \\
$\mathrm{C}(15)$ & $2842(2)$ & $7431(1)$ & $-10(1)$ & $28(1)$ \\
$\mathrm{C}(16)$ & $4340(2)$ & $6702(1)$ & $-590(1)$ & $24(1)$ \\
$\mathrm{C}(17)$ & $6406(2)$ & $6646(1)$ & $261(1)$ & $25(1)$ \\
$\mathrm{C}(18)$ & $5382(2)$ & $6594(1)$ & $1344(1)$ & $22(1)$ \\
& & & & \\
\hline
\end{tabular}

Table S14. Bond lengths $[\AA]$ and angles $\left[{ }^{\circ}\right]$ for TPyE.

\begin{tabular}{ll}
\hline $\mathrm{N}(1)-\mathrm{C}(1)$ & $1.4027(15)$ \\
$\mathrm{N}(1)-\mathrm{C}(6)$ & $1.4422(16)$ \\
$\mathrm{N}(1)-\mathrm{C}(3)$ & $1.4489(15)$ \\
$\mathrm{N}(2)-\mathrm{C}(1)$ & $1.4055(15)$ \\
$\mathrm{N}(2)-\mathrm{C}(10)$ & $1.4393(16)$ \\
$\mathrm{N}(2)-\mathrm{C}(7)$ & $1.4568(15)$ \\
$\mathrm{N}(3)-\mathrm{C}(2)$ & $1.4044(15)$ \\
$\mathrm{N}(3)-\mathrm{C}(11)$ & $1.4541(14)$ \\
$\mathrm{N}(3)-\mathrm{C}(14)$ & $1.4656(16)$ \\
$\mathrm{N}(4)-\mathrm{C}(2)$ & $1.3948(15)$ \\
$\mathrm{N}(4)-\mathrm{C}(15)$ & $1.4457(16)$ \\
$\mathrm{N}(4)-\mathrm{C}(18)$ & $1.4499(15)$ \\
$\mathrm{C}(1)-\mathrm{C}(2)$ & $1.3556(17)$ \\
$\mathrm{C}(3)-\mathrm{C}(4)$ & $1.5276(17)$ \\
$\mathrm{C}(4)-\mathrm{C}(5)$ & $1.5269(19)$ \\
$\mathrm{C}(5)-\mathrm{C}(6)$ & $1.5288(18)$ \\
$\mathrm{C}(7)-\mathrm{C}(8)$ & $1.5252(17)$ \\
$\mathrm{C}(8)-\mathrm{C}(9)$ & $1.5282(18)$ \\
$\mathrm{C}(9)-\mathrm{C}(10)$ & $1.5282(17)$
\end{tabular}




\begin{tabular}{|c|c|}
\hline$C(11)-C(12)$ & $1.5163(17)$ \\
\hline $\mathrm{C}(12)-\mathrm{C}(13)$ & $1.526(2)$ \\
\hline C(13)-C(14) & $1.5322(18)$ \\
\hline$C(15)-C(16)$ & $1.5184(17)$ \\
\hline$C(16)-C(17)$ & $1.5241(18)$ \\
\hline $\mathrm{C}(17)-\mathrm{C}(18)$ & $1.5280(17)$ \\
\hline $\mathrm{C}(1)-\mathrm{N}(1)-\mathrm{C}(6)$ & $124.11(10)$ \\
\hline $\mathrm{C}(1)-\mathrm{N}(1)-\mathrm{C}(3)$ & $122.06(10)$ \\
\hline $\mathrm{C}(6)-\mathrm{N}(1)-\mathrm{C}(3)$ & $113.33(10)$ \\
\hline $\mathrm{C}(1)-\mathrm{N}(2)-\mathrm{C}(10)$ & $124.55(10)$ \\
\hline $\mathrm{C}(1)-\mathrm{N}(2)-\mathrm{C}(7)$ & $121.98(10)$ \\
\hline $\mathrm{C}(10)-\mathrm{N}(2)-\mathrm{C}(7)$ & $113.44(10)$ \\
\hline $\mathrm{C}(2)-\mathrm{N}(3)-\mathrm{C}(11)$ & $122.94(10)$ \\
\hline $\mathrm{C}(2)-\mathrm{N}(3)-\mathrm{C}(14)$ & $119.77(10)$ \\
\hline $\mathrm{C}(11)-\mathrm{N}(3)-\mathrm{C}(14)$ & $110.22(9)$ \\
\hline $\mathrm{C}(2)-\mathrm{N}(4)-\mathrm{C}(15)$ & $123.72(10)$ \\
\hline $\mathrm{C}(2)-\mathrm{N}(4)-\mathrm{C}(18)$ & $123.44(10)$ \\
\hline $\mathrm{C}(15)-\mathrm{N}(4)-\mathrm{C}(18)$ & $112.76(10)$ \\
\hline $\mathrm{C}(2)-\mathrm{C}(1)-\mathrm{N}(1)$ & $122.00(11)$ \\
\hline $\mathrm{C}(2)-\mathrm{C}(1)-\mathrm{N}(2)$ & $122.31(11)$ \\
\hline $\mathrm{N}(1)-\mathrm{C}(1)-\mathrm{N}(2)$ & $115.69(10)$ \\
\hline $\mathrm{C}(1)-\mathrm{C}(2)-\mathrm{N}(4)$ & $122.31(11)$ \\
\hline $\mathrm{C}(1)-\mathrm{C}(2)-\mathrm{N}(3)$ & $124.45(11)$ \\
\hline $\mathrm{N}(4)-\mathrm{C}(2)-\mathrm{N}(3)$ & $113.24(10)$ \\
\hline $\mathrm{N}(1)-\mathrm{C}(3)-\mathrm{C}(4)$ & $103.25(10)$ \\
\hline $\mathrm{C}(5)-\mathrm{C}(4)-\mathrm{C}(3)$ & $103.10(10)$ \\
\hline$C(4)-C(5)-C(6)$ & $102.95(10)$ \\
\hline $\mathrm{N}(1)-\mathrm{C}(6)-\mathrm{C}(5)$ & $103.26(11)$ \\
\hline $\mathrm{N}(2)-\mathrm{C}(7)-\mathrm{C}(8)$ & $103.41(10)$ \\
\hline $\mathrm{C}(7)-\mathrm{C}(8)-\mathrm{C}(9)$ & $103.56(10)$ \\
\hline $\mathrm{C}(8)-\mathrm{C}(9)-\mathrm{C}(10)$ & $103.47(10)$ \\
\hline $\mathrm{N}(2)-\mathrm{C}(10)-\mathrm{C}(9)$ & 103.41(10) \\
\hline $\mathrm{N}(3)-\mathrm{C}(11)-\mathrm{C}(12)$ & $101.96(10)$ \\
\hline $\mathrm{C}(11)-\mathrm{C}(12)-\mathrm{C}(13)$ & $102.99(10)$ \\
\hline $\mathrm{C}(12)-\mathrm{C}(13)-\mathrm{C}(14)$ & 104.93(11) \\
\hline $\mathrm{N}(3)-\mathrm{C}(14)-\mathrm{C}(13)$ & $104.45(10)$ \\
\hline $\mathrm{N}(4)-\mathrm{C}(15)-\mathrm{C}(16)$ & $104.12(10)$ \\
\hline$C(15)-C(16)-C(17)$ & $103.41(10)$ \\
\hline $\mathrm{C}(16)-\mathrm{C}(17)-\mathrm{C}(18)$ & $102.90(10)$ \\
\hline $\mathrm{N}(4)-\mathrm{C}(18)-\mathrm{C}(17)$ & $102.86(9)$ \\
\hline
\end{tabular}

Symmetry transformations used to generate equivalent atoms:

Table S15. Anisotropic displacement parameters $\left(\AA^{2} \times 10^{3}\right)$ for TPyE. The anisotropic displacement factor exponent takes the form: $-2 \pi^{2}\left[h^{2} a^{* 2} U^{11}+\ldots+2 h k a^{*} b^{*} U^{12}\right]$

\begin{tabular}{lcccccc}
\hline & $\mathrm{U}^{11}$ & $\mathrm{U}^{22}$ & $\mathrm{U}^{33}$ & $\mathrm{U}^{23}$ & $\mathrm{U}^{13}$ & $\mathrm{U}^{12}$ \\
\hline $\mathrm{N}(1)$ & $27(1)$ & $25(1)$ & $21(1)$ & $-5(1)$ & $9(1)$ & $-6(1)$ \\
$\mathrm{N}(2)$ & $38(1)$ & $18(1)$ & $20(1)$ & $1(1)$ & $-9(1)$ & $4(1)$ \\
$\mathrm{N}(3)$ & $19(1)$ & $19(1)$ & $31(1)$ & $4(1)$ & $10(1)$ & $6(1)$ \\
$\mathrm{N}(4)$ & $40(1)$ & $40(1)$ & $18(1)$ & $7(1)$ & $8(1)$ & $28(1)$ \\
$\mathrm{C}(1)$ & $24(1)$ & $19(1)$ & $19(1)$ & $-1(1)$ & $3(1)$ & $4(1)$ \\
$\mathrm{C}(2)$ & $22(1)$ & $20(1)$ & $22(1)$ & $1(1)$ & $5(1)$ & $7(1)$ \\
& & & & & & \\
& & & & & &
\end{tabular}




\begin{tabular}{lcccccc}
$\mathrm{C}(3)$ & $22(1)$ & $24(1)$ & $24(1)$ & $-1(1)$ & $7(1)$ & $-1(1)$ \\
$\mathrm{C}(4)$ & $30(1)$ & $26(1)$ & $25(1)$ & $-2(1)$ & $10(1)$ & $2(1)$ \\
$\mathrm{C}(5)$ & $30(1)$ & $45(1)$ & $24(1)$ & $-10(1)$ & $0(1)$ & $0(1)$ \\
$\mathrm{C}(6)$ & $20(1)$ & $37(1)$ & $29(1)$ & $-10(1)$ & $4(1)$ & $-3(1)$ \\
$\mathrm{C}(7)$ & $23(1)$ & $24(1)$ & $20(1)$ & $2(1)$ & $-4(1)$ & $4(1)$ \\
$\mathrm{C}(8)$ & $27(1)$ & $23(1)$ & $24(1)$ & $3(1)$ & $-2(1)$ & $8(1)$ \\
$\mathrm{C}(9)$ & $30(1)$ & $21(1)$ & $34(1)$ & $4(1)$ & $-8(1)$ & $-1(1)$ \\
$\mathrm{C}(10)$ & $23(1)$ & $21(1)$ & $27(1)$ & $2(1)$ & $-6(1)$ & $3(1)$ \\
$\mathrm{C}(11)$ & $17(1)$ & $22(1)$ & $29(1)$ & $1(1)$ & $7(1)$ & $6(1)$ \\
$\mathrm{C}(12)$ & $28(1)$ & $25(1)$ & $26(1)$ & $3(1)$ & $3(1)$ & $13(1)$ \\
$\mathrm{C}(13)$ & $36(1)$ & $21(1)$ & $58(1)$ & $5(1)$ & $18(1)$ & $3(1)$ \\
$\mathrm{C}(14)$ & $19(1)$ & $25(1)$ & $28(1)$ & $1(1)$ & $6(1)$ & $2(1)$ \\
$\mathrm{C}(15)$ & $25(1)$ & $41(1)$ & $19(1)$ & $-2(1)$ & $0(1)$ & $14(1)$ \\
$\mathrm{C}(16)$ & $25(1)$ & $28(1)$ & $21(1)$ & $-3(1)$ & $6(1)$ & $2(1)$ \\
$\mathrm{C}(17)$ & $21(1)$ & $27(1)$ & $29(1)$ & $2(1)$ & $8(1)$ & $7(1)$ \\
$\mathrm{C}(18)$ & $21(1)$ & $23(1)$ & $23(1)$ & $3(1)$ & $3(1)$ & $10(1)$ \\
& & & & & & \\
\hline
\end{tabular}

Table S16. Torsion angles $\left[{ }^{\circ}\right]$ for TPyE.

\begin{tabular}{|c|c|}
\hline $\mathrm{C}(6)-\mathrm{N}(1)-\mathrm{C}(1)-\mathrm{C}(2)$ & $-58.75(18)$ \\
\hline $\mathrm{C}(3)-\mathrm{N}(1)-\mathrm{C}(1)-\mathrm{C}(2)$ & $129.93(14)$ \\
\hline $\mathrm{C}(6)-\mathrm{N}(1)-\mathrm{C}(1)-\mathrm{N}(2)$ & $120.89(14)$ \\
\hline $\mathrm{C}(3)-\mathrm{N}(1)-\mathrm{C}(1)-\mathrm{N}(2)$ & $-50.42(16)$ \\
\hline $\mathrm{C}(10)-\mathrm{N}(2)-\mathrm{C}(1)-\mathrm{C}(2)$ & $-51.11(19)$ \\
\hline $\mathrm{C}(7)-\mathrm{N}(2)-\mathrm{C}(1)-\mathrm{C}(2)$ & $126.87(14)$ \\
\hline $\mathrm{C}(10)-\mathrm{N}(2)-\mathrm{C}(1)-\mathrm{N}(1)$ & $129.25(13)$ \\
\hline $\mathrm{C}(7)-\mathrm{N}(2)-\mathrm{C}(1)-\mathrm{N}(1)$ & $-52.77(17)$ \\
\hline $\mathrm{N}(1)-\mathrm{C}(1)-\mathrm{C}(2)-\mathrm{N}(4)$ & $157.77(12)$ \\
\hline $\mathrm{N}(2)-\mathrm{C}(1)-\mathrm{C}(2)-\mathrm{N}(4)$ & $-21.8(2)$ \\
\hline $\mathrm{N}(1)-\mathrm{C}(1)-\mathrm{C}(2)-\mathrm{N}(3)$ & $-22.4(2)$ \\
\hline $\mathrm{N}(2)-\mathrm{C}(1)-\mathrm{C}(2)-\mathrm{N}(3)$ & $157.94(12)$ \\
\hline $\mathrm{C}(15)-\mathrm{N}(4)-\mathrm{C}(2)-\mathrm{C}(1)$ & $144.56(14)$ \\
\hline $\mathrm{C}(18)-\mathrm{N}(4)-\mathrm{C}(2)-\mathrm{C}(1)$ & $-39.0(2)$ \\
\hline $\mathrm{C}(15)-\mathrm{N}(4)-\mathrm{C}(2)-\mathrm{N}(3)$ & $-35.25(19)$ \\
\hline C(18)-N(4)-C(2)-N(3) & $141.17(13)$ \\
\hline $\mathrm{C}(11)-\mathrm{N}(3)-\mathrm{C}(2)-\mathrm{C}(1)$ & $-31.46(19)$ \\
\hline $\mathrm{C}(14)-\mathrm{N}(3)-\mathrm{C}(2)-\mathrm{C}(1)$ & $116.21(14)$ \\
\hline C(11)-N(3)-C(2)-N(4) & $148.36(12)$ \\
\hline C(14)-N(3)-C(2)-N(4) & $-63.98(15)$ \\
\hline $\mathrm{C}(1)-\mathrm{N}(1)-\mathrm{C}(3)-\mathrm{C}(4)$ & $-176.89(11)$ \\
\hline $\mathrm{C}(6)-\mathrm{N}(1)-\mathrm{C}(3)-\mathrm{C}(4)$ & $10.93(14)$ \\
\hline $\mathrm{N}(1)-\mathrm{C}(3)-\mathrm{C}(4)-\mathrm{C}(5)$ & $-29.89(13)$ \\
\hline$C(3)-C(4)-C(5)-C(6)$ & $37.68(14)$ \\
\hline $\mathrm{C}(1)-\mathrm{N}(1)-\mathrm{C}(6)-\mathrm{C}(5)$ & $-159.35(12)$ \\
\hline $\mathrm{C}(3)-\mathrm{N}(1)-\mathrm{C}(6)-\mathrm{C}(5)$ & $12.64(15)$ \\
\hline $\mathrm{C}(4)-\mathrm{C}(5)-\mathrm{C}(6)-\mathrm{N}(1)$ & $-30.86(14)$ \\
\hline $\mathrm{C}(1)-\mathrm{N}(2)-\mathrm{C}(7)-\mathrm{C}(8)$ & $-168.66(11)$ \\
\hline $\mathrm{C}(10)-\mathrm{N}(2)-\mathrm{C}(7)-\mathrm{C}(8)$ & $9.53(15)$ \\
\hline $\mathrm{N}(2)-\mathrm{C}(7)-\mathrm{C}(8)-\mathrm{C}(9)$ & $-27.88(13)$ \\
\hline$C(7)-C(8)-C(9)-C(10)$ & $35.87(13)$ \\
\hline $\mathrm{C}(1)-\mathrm{N}(2)-\mathrm{C}(10)-\mathrm{C}(9)$ & $-168.99(12)$ \\
\hline $\mathrm{C}(7)-\mathrm{N}(2)-\mathrm{C}(10)-\mathrm{C}(9)$ & $12.87(15)$ \\
\hline $\mathrm{C}(8)-\mathrm{C}(9)-\mathrm{C}(10)-\mathrm{N}(2)$ & $-29.83(14)$ \\
\hline $\mathrm{C}(2)-\mathrm{N}(3)-\mathrm{C}(11)-\mathrm{C}(12)$ & $-175.71(11)$ \\
\hline
\end{tabular}


C(14)-N(3)-C(11)-C(12)

$33.95(13)$

$\mathrm{N}(3)-\mathrm{C}(11)-\mathrm{C}(12)-\mathrm{C}(13)$

$-39.02(13)$

$\mathrm{C}(11)-\mathrm{C}(12)-\mathrm{C}(13)-\mathrm{C}(14)$

$30.87(14)$

$\mathrm{C}(2)-\mathrm{N}(3)-\mathrm{C}(14)-\mathrm{C}(13)$

$-165.97(11)$

$\mathrm{C}(11)-\mathrm{N}(3)-\mathrm{C}(14)-\mathrm{C}(13)$

$-14.55(14)$

$\mathrm{C}(12)-\mathrm{C}(13)-\mathrm{C}(14)-\mathrm{N}(3)$

$-10.87(14)$

$\mathrm{C}(2)-\mathrm{N}(4)-\mathrm{C}(15)-\mathrm{C}(16)$

$-176.23(13)$

$\mathrm{C}(18)-\mathrm{N}(4)-\mathrm{C}(15)-\mathrm{C}(16)$

7.01(16)

$\mathrm{N}(4)-\mathrm{C}(15)-\mathrm{C}(16)-\mathrm{C}(17)$

$-27.44(14)$

$\mathrm{C}(15)-\mathrm{C}(16)-\mathrm{C}(17)-\mathrm{C}(18)$

$37.22(13)$

$\mathrm{C}(2)-\mathrm{N}(4)-\mathrm{C}(18)-\mathrm{C}(17)$

$-160.50(13)$

$\mathrm{C}(15)-\mathrm{N}(4)-\mathrm{C}(18)-\mathrm{C}(17)$

$16.27(15)$

$\mathrm{C}(16)-\mathrm{C}(17)-\mathrm{C}(18)-\mathrm{N}(4)$

$-32.58(13)$ 
X-Ray Diffraction of TME: Crystals suitable for X-ray diffraction were grown by heating a supersaturated mixture of TME in DMAc at $80^{\circ} \mathrm{C}$. Once all TME had dissolved, the solution was allowed to cool slowly to room temperature, resulting in colorless crystals. Low-temperature diffraction data ( $\omega$-scans) were collected on a Rigaku MicroMax-007HF diffractometer coupled to a Dectris Pilatus3R detector with Mo $\mathrm{K} \alpha(\lambda=0.71073 \AA)$ for the structure of TME. The diffraction images were processed and scaled using Rigaku Oxford Diffraction software. ${ }^{21}$ The structure was solved with SHELXT and was refined against $\mathrm{F}^{2}$ on all data by full-matrix least squares with SHELXL. ${ }^{22}$ All non-hydrogen atoms were refined anisotropically. Hydrogen atoms were included in the model at geometrically calculated positions and refined using a riding model. The isotropic displacement parameters of all hydrogen atoms were fixed to 1.2 times the $U$ value of the atoms to which they are linked (1.5 times for methyl groups). The full numbering scheme can be found in the full details of the X-ray structure determination (CIF), which is included as Supporting Information. CCDC number 2111618 contains the supplementary crystallographic data for this paper.

Table S17. Crystal data and structure refinement for TME.

\begin{tabular}{|c|c|c|}
\hline Empirical formula & C18 H32 N4 O4 & \\
\hline Formula weight & 368.47 & \\
\hline Temperature & $93(2) \mathrm{K}$ & \\
\hline Wavelength & $0.71073 \AA$ & \\
\hline Crystal system & Monoclinic & \\
\hline Space group & $\mathrm{P} 2 / \mathrm{c}$ & \\
\hline Unit cell dimensions & $\mathrm{a}=11.8750(10) \AA$ & $\alpha=90^{\circ}$ \\
\hline & $\mathrm{b}=6.0835(2) \AA$ & $\beta=134.314(14)^{\circ}$ \\
\hline & $\mathrm{c}=18.3743(15) \AA$ & $\gamma=90^{\circ}$ \\
\hline Volume & $949.8(2) \AA^{3}$ & \\
\hline Z & 2 & \\
\hline Density (calculated) & $1.288 \mathrm{Mg} / \mathrm{m}^{3}$ & \\
\hline Absorption coefficient & $0.092 \mathrm{~mm}^{-1}$ & \\
\hline$F(000)$ & 400 & \\
\hline Crystal size & $0.500 \times 0.500 \times 0.1$ & \\
\hline Crystal color and habit & colorless block & \\
\hline Diffractometer & Dectris Pilatus 3R & \\
\hline Theta range for data collection & 3.099 to $28.278^{\circ}$. & \\
\hline Index ranges & $-15<=\mathrm{h}<=15,-8<=1$ & $=1<=24$ \\
\hline Reflections collected & 18066 & \\
\hline Independent reflections & $2354[\mathrm{R}(\mathrm{int})=0.02$ & \\
\hline Observed reflections $(\mathrm{I}>2 \operatorname{sigma}(\mathrm{I}))$ & 2153 & \\
\hline Completeness to theta $=25.242^{\circ}$ & $99.9 \%$ & \\
\hline Absorption correction & Semi-empirical fro & \\
\hline Max. and min. transmission & 1.00000 and 0.589 & \\
\hline Solution method & SHELXT-2014/5 & 014) \\
\hline Refinement method & SHELXL-2014/7 & 014) \\
\hline Data / restraints / parameters & $2354 / 0 / 118$ & \\
\hline Goodness-of-fit on $\mathrm{F}^{2}$ & 1.050 & \\
\hline
\end{tabular}


Final $\mathrm{R}$ indices [I $>2 \operatorname{sigma}(\mathrm{I})]$

$\mathrm{R}$ indices (all data)

Extinction coefficient

Largest diff. peak and hole
$\mathrm{R} 1=0.0355, \mathrm{wR} 2=0.0920$

$\mathrm{R} 1=0.0385, \mathrm{wR} 2=0.0940$

$\mathrm{n} / \mathrm{a}$

0.371 and -0.217 e. $\AA^{-3}$

Table S18. Atomic coordinates $\left(\times 10^{4}\right)$ and equivalent isotropic displacement parameters $\left(\AA^{2} \times 10^{3}\right)$ for TME. $U(e q)$ is defined as one third of the trace of the orthogonalized $\mathrm{Uij}^{\mathrm{ij}}$ tensor.

\begin{tabular}{lcrrr}
\hline & $\mathrm{x}$ & $\mathrm{y}$ & $\mathrm{z}$ & $\mathrm{U}(\mathrm{eq})$ \\
\hline $\mathrm{O}(1)$ & & & \\
$\mathrm{O}(2)$ & $9345(1)$ & $757(1)$ & $5851(1)$ & $20(1)$ \\
$\mathrm{N}(1)$ & $7261(1)$ & $3770(1)$ & $1258(1)$ & $16(1)$ \\
$\mathrm{N}(2)$ & $6890(1)$ & $2214(1)$ & $3820(1)$ & $10(1)$ \\
$\mathrm{C}(1)$ & $6411(1)$ & $3229(1)$ & $2359(1)$ & $10(1)$ \\
$\mathrm{C}(2)$ & $5780(1)$ & $2720(1)$ & $2768(1)$ & $9(1)$ \\
$\mathrm{C}(3)$ & $8414(1)$ & $3309(2)$ & $4500(1)$ & $13(1)$ \\
$\mathrm{C}(4)$ & $9182(1)$ & $3027(2)$ & $5590(1)$ & $17(1)$ \\
$\mathrm{C}(5)$ & $7056(1)$ & $-108(2)$ & $4088(1)$ & $15(1)$ \\
$\mathrm{C}(6)$ & $7845(1)$ & $-294(2)$ & $5181(1)$ & $19(1)$ \\
$\mathrm{C}(7)$ & $5545(1)$ & $4721(2)$ & $1495(1)$ & $13(1)$ \\
$\mathrm{C}(8)$ & $6635(1)$ & $5553(2)$ & $1392(1)$ & $15(1)$ \\
$\mathrm{C}(9)$ & $7103(1)$ & $1415(2)$ & $2256(1)$ & $12(1)$ \\
& $8130(1)$ & $2324(2)$ & $2114(1)$ & $15(1)$ \\
\hline
\end{tabular}

Table S19. Bond lengths $[\AA]]$ and angles $\left[{ }^{\circ}\right]$ for TME.

\begin{tabular}{ll}
\hline $\mathrm{O}(1)-\mathrm{C}(5)$ & $1.4265(12)$ \\
$\mathrm{O}(1)-\mathrm{C}(3)$ & $1.4302(12)$ \\
$\mathrm{O}(2)-\mathrm{C}(7)$ & $1.4300(11)$ \\
$\mathrm{O}(2)-\mathrm{C}(9)$ & $1.4309(11)$ \\
$\mathrm{N}(1)-\mathrm{C}(1)$ & $1.4169(11)$ \\
$\mathrm{N}(1)-\mathrm{C}(2)$ & $1.4563(11)$ \\
$\mathrm{N}(1)-\mathrm{C}(4)$ & $1.4630(11)$ \\
$\mathrm{N}(2)-\mathrm{C}(1)$ & $1.4182(10)$ \\
$\mathrm{N}(2)-\mathrm{C}(6)$ & $1.4560(11)$ \\
$\mathrm{N}(2)-\mathrm{C}(8)$ & $1.4653(11)$ \\
$\mathrm{C}(1)-\mathrm{C}(1) \# 1$ & $1.3616(16)$ \\
$\mathrm{C}(2)-\mathrm{C}(3)$ & $1.5235(12)$ \\
$\mathrm{C}(4)-\mathrm{C}(5)$ & $1.5155(13)$ \\
$\mathrm{C}(6)-\mathrm{C}(7)$ & $1.5202(12)$ \\
$\mathrm{C}(8)-\mathrm{C}(9)$ & $1.5172(12)$ \\
$\mathrm{C}(5)-\mathrm{O}(1)-\mathrm{C}(3)$ & \\
$\mathrm{C}(7)-\mathrm{O}(2)-\mathrm{C}(9)$ & $110.38(7)$ \\
$\mathrm{C}(1)-\mathrm{N}(1)-\mathrm{C}(2)$ & $109.84(7)$ \\
$\mathrm{C}(1)-\mathrm{N}(1)-\mathrm{C}(4)$ & $118.98(7)$ \\
$\mathrm{C}(2)-\mathrm{N}(1)-\mathrm{C}(4)$ & $116.52(7)$ \\
$\mathrm{C}(1)-\mathrm{N}(2)-\mathrm{C}(6)$ & $111.03(7)$ \\
$\mathrm{C}(1)-\mathrm{N}(2)-\mathrm{C}(8)$ & $118.83(7)$ \\
$\mathrm{C}(6)-\mathrm{N}(2)-\mathrm{C}(8)$ & $117.12(7)$ \\
$\mathrm{C}(1) \# 1-\mathrm{C}(1)-\mathrm{N}(1)$ & $111.21(7)$ \\
$\mathrm{C}(1) \# 1-\mathrm{C}(1)-\mathrm{N}(2)$ & $119.11(9)$ \\
$\mathrm{N}(1)-\mathrm{C}(1)-\mathrm{N}(2)$ & $124.86(9)$ \\
\end{tabular}




$\begin{array}{ll}\mathrm{N}(1)-\mathrm{C}(2)-\mathrm{C}(3) & 108.64(7) \\ \mathrm{O}(1)-\mathrm{C}(3)-\mathrm{C}(2) & 111.50(8) \\ \mathrm{N}(1)-\mathrm{C}(4)-\mathrm{C}(5) & 109.31(8) \\ \mathrm{O}(1)-\mathrm{C}(5)-\mathrm{C}(4) & 110.71(8) \\ \mathrm{N}(2)-\mathrm{C}(6)-\mathrm{C}(7) & 109.20(7) \\ \mathrm{O}(2)-\mathrm{C}(7)-\mathrm{C}(6) & 111.05(7) \\ \mathrm{N}(2)-\mathrm{C}(8)-\mathrm{C}(9) & 109.77(7) \\ \mathrm{O}(2)-\mathrm{C}(9)-\mathrm{C}(8) & 111.34(7)\end{array}$

Symmetry transformations used to generate equivalent atoms:

$\# 1-\mathrm{x}+1, \mathrm{y},-\mathrm{z}+1 / 2$

Table S20. Anisotropic displacement parameters $\left(\AA^{2} \times 10^{3}\right)$ for TME. The anisotropic displacement factor exponent takes the form: $-2 \pi^{2}\left[h^{2} a^{* 2} U^{11}+\ldots+2 h k a^{*} b^{*} U^{12}\right]$

\begin{tabular}{lcccccc}
\hline & $\mathrm{U}^{11}$ & $\mathrm{U}^{22}$ & $\mathrm{U}^{33}$ & $\mathrm{U}^{23}$ & $\mathrm{U}^{13}$ & $\mathrm{U}^{12}$ \\
\hline $\mathrm{O}(1)$ & $15(1)$ & $26(1)$ & $11(1)$ & $6(1)$ & $6(1)$ & $5(1)$ \\
$\mathrm{O}(2)$ & $19(1)$ & $20(1)$ & $16(1)$ & $2(1)$ & $15(1)$ & $3(1)$ \\
$\mathrm{N}(1)$ & $8(1)$ & $11(1)$ & $7(1)$ & $1(1)$ & $4(1)$ & $-1(1)$ \\
$\mathrm{N}(2)$ & $11(1)$ & $11(1)$ & $10(1)$ & $3(1)$ & $8(1)$ & $3(1)$ \\
$\mathrm{C}(1)$ & $10(1)$ & $9(1)$ & $8(1)$ & $1(1)$ & $6(1)$ & $0(1)$ \\
$\mathrm{C}(2)$ & $9(1)$ & $16(1)$ & $9(1)$ & $-1(1)$ & $5(1)$ & $-2(1)$ \\
$\mathrm{C}(3)$ & $14(1)$ & $23(1)$ & $10(1)$ & $-1(1)$ & $6(1)$ & $-1(1)$ \\
$\mathrm{C}(4)$ & $15(1)$ & $12(1)$ & $13(1)$ & $3(1)$ & $9(1)$ & $0(1)$ \\
$\mathrm{C}(5)$ & $19(1)$ & $21(1)$ & $16(1)$ & $7(1)$ & $12(1)$ & $3(1)$ \\
$\mathrm{C}(6)$ & $14(1)$ & $14(1)$ & $14(1)$ & $5(1)$ & $11(1)$ & $4(1)$ \\
$\mathrm{C}(7)$ & $19(1)$ & $15(1)$ & $16(1)$ & $2(1)$ & $14(1)$ & $1(1)$ \\
$\mathrm{C}(8)$ & $13(1)$ & $13(1)$ & $13(1)$ & $1(1)$ & $10(1)$ & $3(1)$ \\
$\mathrm{C}(9)$ & $13(1)$ & $19(1)$ & $15(1)$ & $1(1)$ & $11(1)$ & $2(1)$ \\
& & & & & & \\
\hline
\end{tabular}

Table S21. Torsion angles $\left[^{\circ}\right]$ for TME.

\begin{tabular}{lc}
\hline $\mathrm{C}(2)-\mathrm{N}(1)-\mathrm{C}(1)-\mathrm{C}(1) \# 1$ & $-145.90(6)$ \\
$\mathrm{C}(4)-\mathrm{N}(1)-\mathrm{C}(1)-\mathrm{C}(1) \# 1$ & $76.82(8)$ \\
$\mathrm{C}(2)-\mathrm{N}(1)-\mathrm{C}(1)-\mathrm{N}(2)$ & $33.16(11)$ \\
$\mathrm{C}(4)-\mathrm{N}(1)-\mathrm{C}(1)-\mathrm{N}(2)$ & $-104.13(9)$ \\
$\mathrm{C}(6)-\mathrm{N}(2)-\mathrm{C}(1)-\mathrm{C}(1) \# 1$ & $28.34(10)$ \\
$\mathrm{C}(8)-\mathrm{N}(2)-\mathrm{C}(1)-\mathrm{C}(1) \# 1$ & $-109.90(6)$ \\
$\mathrm{C}(6)-\mathrm{N}(2)-\mathrm{C}(1)-\mathrm{N}(1)$ & $-150.65(8)$ \\
$\mathrm{C}(8)-\mathrm{N}(2)-\mathrm{C}(1)-\mathrm{N}(1)$ & $71.11(10)$ \\
$\mathrm{C}(1)-\mathrm{N}(1)-\mathrm{C}(2)-\mathrm{C}(3)$ & $163.53(8)$ \\
$\mathrm{C}(4)-\mathrm{N}(1)-\mathrm{C}(2)-\mathrm{C}(3)$ & $-57.03(9)$ \\
$\mathrm{C}(5)-\mathrm{O}(1)-\mathrm{C}(3)-\mathrm{C}(2)$ & $-58.81(10)$ \\
$\mathrm{N}(1)-\mathrm{C}(2)-\mathrm{C}(3)-\mathrm{O}(1)$ & $57.39(10)$ \\
$\mathrm{C}(1)-\mathrm{N}(1)-\mathrm{C}(4)-\mathrm{C}(5)$ & $-161.58(7)$ \\
$\mathrm{C}(2)-\mathrm{N}(1)-\mathrm{C}(4)-\mathrm{C}(5)$ & $57.90(9)$ \\
$\mathrm{C}(3)-\mathrm{O}(1)-\mathrm{C}(5)-\mathrm{C}(4)$ & $58.84(10)$ \\
$\mathrm{N}(1)-\mathrm{C}(4)-\mathrm{C}(5)-\mathrm{O}(1)$ & $-58.14(10)$ \\
$\mathrm{C}(1)-\mathrm{N}(2)-\mathrm{C}(6)-\mathrm{C}(7)$ & $163.14(7)$ \\
$\mathrm{C}(8)-\mathrm{N}(2)-\mathrm{C}(6)-\mathrm{C}(7)$ & $-56.35(9)$ \\
$\mathrm{C}(9)-\mathrm{O}(2)-\mathrm{C}(7)-\mathrm{C}(6)$ & $-59.84(9)$ \\
$\mathrm{N}(2)-\mathrm{C}(6)-\mathrm{C}(7)-\mathrm{O}(2)$ & $58.57(9)$
\end{tabular}


$\mathrm{C}(1)-\mathrm{N}(2)-\mathrm{C}(8)-\mathrm{C}(9)$

$-163.17(7)$

$\mathrm{C}(6)-\mathrm{N}(2)-\mathrm{C}(8)-\mathrm{C}(9)$

55.57(9)

$\mathrm{C}(7)-\mathrm{O}(2)-\mathrm{C}(9)-\mathrm{C}(8)$

$58.86(10)$

$\mathrm{N}(2)-\mathrm{C}(8)-\mathrm{C}(9)-\mathrm{O}(2)$

$-56.56(9)$

Symmetry transformations used to generate equivalent atoms:

\#1 - $\mathrm{x}+1, \mathrm{y},-\mathrm{z}+1 / 2$ 
X-Ray Diffraction of TAzE: Crystals suitable for X-ray diffraction were grown by heating a supersaturated mixture of TAzE in DMAc at $80^{\circ} \mathrm{C}$. Once all TAzE had dissolved, the solution was allowed to cool slowly to room temperature, resulting in colorless crystals. Low-temperature diffraction data ( $\omega$-scans) were collected on a Rigaku MicroMax-007HF diffractometer coupled to a Saturn994+ CCD detector with $\mathrm{Cu} \mathrm{K \alpha}(\lambda=1.54178 \AA)$ for the structure of TAzE. The diffraction images were processed and scaled using Rigaku Oxford Diffraction software. ${ }^{21}$ The structure was solved with SHELXT and was refined against $\mathrm{F}^{2}$ on all data by full-matrix least squares with SHELXL. ${ }^{22}$ All non-hydrogen atoms were refined anisotropically. Hydrogen atoms were included in the model at geometrically calculated positions and refined using a riding model. The isotropic displacement parameters of all hydrogen atoms were fixed to 1.2 times the $U$ value of the atoms to which they are linked (1.5 times for methyl groups). The full numbering scheme can be found in the full details of the X-ray structure determination (CIF), which is included as Supporting Information. CCDC number 2111619 contains the supplementary crystallographic data for this paper.

Table S22. Crystal data and structure refinement for TAzE.

Empirical formula

Formula weight

Temperature

Wavelength

Crystal system

Space group

Unit cell dimensions

Volume

Z

Density (calculated)

Absorption coefficient

$\mathrm{F}(000)$

Crystal size

Crystal color and habit

Diffractometer

Theta range for data collection

Index ranges

Reflections collected

Independent reflections

Observed reflections (I > 2sigma(I))

Completeness to theta $=66.598^{\circ}$

Absorption correction

Max. and min. transmission

Solution method

Refinement method

Data / restraints / parameters
C104 H192 N16

1666.72

93(2) K

$1.54184 \AA$

Monoclinic

$\mathrm{P} 2 / \mathrm{c}$

$\mathrm{a}=20.0666(5) \AA$

$\alpha=90^{\circ}$

$\mathrm{b}=6.57270(10) \AA$

$\beta=91.303(2)^{\circ}$

$\mathrm{c}=18.5611(4) \AA$

2447.42(9) $\AA^{3}$

1

$1.131 \mathrm{Mg} / \mathrm{m}^{3}$

$0.500 \mathrm{~mm}^{-1}$

928

$0.200 \times 0.080 \times 0.020 \mathrm{~mm}^{3}$

Colorless Plate

Rigaku Saturn 944+ CCD

2.202 to $66.598^{\circ}$.

$-23<=\mathrm{h}<=23,-7<=\mathrm{k}<=7,-22<=\mathrm{l}<=22$

73896

$4292[\mathrm{R}(\mathrm{int})=0.0868]$

3597

$99.3 \%$

Semi-empirical from equivalents

1.00000 and 0.63774

SHELXT-2014/5 (Sheldrick, 2014)

SHELXL-2014/7 (Sheldrick, 2014)

4292 / 0 / 272 
Goodness-of-fit on $\mathrm{F}^{2}$

Final $R$ indices [I $>2 \operatorname{sigma}(\mathrm{I})]$

$\mathrm{R}$ indices (all data)

Extinction coefficient

Largest diff. peak and hole
1.156

$\mathrm{R} 1=0.0960, \mathrm{wR} 2=0.2564$

$\mathrm{R} 1=0.1110, \mathrm{wR} 2=0.2690$

$0.0012(3)$

0.698 and -0.283 e. $\AA^{-3}$

Table S23. Atomic coordinates $\left(\times 10^{4}\right)$ and equivalent isotropic displacement parameters $\left(\AA^{2} \times 10^{3}\right)$ for TAzE. $U(e q)$ is defined as one third of the trace of the orthogonalized $U^{i j}$ tensor.

\begin{tabular}{|c|c|c|c|c|}
\hline & $\mathrm{x}$ & $\mathrm{y}$ & $\mathrm{z}$ & $\mathrm{U}(\mathrm{eq})$ \\
\hline $\mathrm{N}(1)$ & $6984(2)$ & $2524(5)$ & $4193(2)$ & $28(1)$ \\
\hline $\mathrm{N}(2)$ & $6635(2)$ & $2657(5)$ & $5398(2)$ & $26(1)$ \\
\hline $\mathrm{N}(3)$ & $8337(2)$ & $1667(5)$ & $4795(2)$ & $29(1)$ \\
\hline $\mathrm{N}(4)$ & $8032(2)$ & $3548(5)$ & $5817(2)$ & $27(1)$ \\
\hline $\mathrm{C}(1)$ & $7173(2)$ & $2608(6)$ & $4927(2)$ & $27(1)$ \\
\hline $\mathrm{C}(2)$ & $7817(2)$ & $2622(6)$ & $5165(2)$ & $26(1)$ \\
\hline $\mathrm{C}(3)$ & $6546(2)$ & $853(6)$ & $3962(2)$ & $31(1)$ \\
\hline$C(4)$ & $6302(2)$ & $957(6)$ & $3185(2)$ & $31(1)$ \\
\hline$C(5)$ & $5930(2)$ & $2927(7)$ & 2992(2) & $33(1)$ \\
\hline $\mathrm{C}(6)$ & $6386(2)$ & $4643(7)$ & $2755(2)$ & $34(1)$ \\
\hline$C(7)$ & $6877(2)$ & $5406(7)$ & $3333(2)$ & $35(1)$ \\
\hline $\mathrm{C}(8)$ & $7324(2)$ & $3754(6)$ & $3676(2)$ & $30(1)$ \\
\hline $\mathrm{C}(9)$ & $6136(2)$ & $4238(6)$ & $5271(2)$ & $30(1)$ \\
\hline$C(10)$ & $5564(2)$ & $4276(6)$ & $5790(2)$ & $29(1)$ \\
\hline $\mathrm{C}(11)$ & $5131(2)$ & $2352(7)$ & $5792(3)$ & $38(1)$ \\
\hline$C(12)$ & $5410(2)$ & $645(7)$ & $6270(2)$ & $36(1)$ \\
\hline$C(13)$ & $6075(2)$ & $-184(6)$ & $6057(2)$ & $33(1)$ \\
\hline $\mathrm{C}(14)$ & $6639(2)$ & $1377(6)$ & $6036(2)$ & $29(1)$ \\
\hline$C(15)$ & $8179(2)$ & $-173(6)$ & $4396(2)$ & $31(1)$ \\
\hline$C(16)$ & $8773(2)$ & $-1523(7)$ & $4227(3)$ & $38(1)$ \\
\hline$C(17)$ & $9152(2)$ & $-896(7)$ & $3563(3)$ & $42(1)$ \\
\hline $\mathrm{C}(18)$ & $9572(2)$ & $1023(7)$ & $3649(3)$ & $40(1)$ \\
\hline$C(19)$ & $9192(2)$ & 2891(7) & $3914(3)$ & $40(1)$ \\
\hline$C(20)$ & $8962(2)$ & $2742(7)$ & $4676(2)$ & $35(1)$ \\
\hline $\mathrm{C}(21)$ & $8346(2)$ & $2272(6)$ & $6375(2)$ & $31(1)$ \\
\hline $\mathrm{C}(22)$ & $9052(2)$ & $2900(7)$ & $6583(2)$ & $33(1)$ \\
\hline $\mathrm{C}(23)$ & $9117(2)$ & $4905(7)$ & $7002(2)$ & $36(1)$ \\
\hline $\mathrm{C}(24)$ & $8751(2)$ & $6695(6)$ & $6671(2)$ & $34(1)$ \\
\hline$C(25)$ & $8000(2)$ & $6475(6)$ & $6681(2)$ & $32(1)$ \\
\hline$C(26)$ & $7677(2)$ & $5372(6)$ & $6042(2)$ & $28(1)$ \\
\hline
\end{tabular}

Table S24. Bond lengths $[\AA]$ and angles $\left[{ }^{\circ}\right]$ for TAzE.

\begin{tabular}{ll}
\hline $\mathrm{N}(1)-\mathrm{C}(1)$ & $1.407(5)$ \\
$\mathrm{N}(1)-\mathrm{C}(8)$ & $1.439(5)$ \\
$\mathrm{N}(1)-\mathrm{C}(3)$ & $1.465(5)$ \\
$\mathrm{N}(2)-\mathrm{C}(1)$ & $1.405(5)$ \\
$\mathrm{N}(2)-\mathrm{C}(14)$ & $1.453(5)$ \\
$\mathrm{N}(2)-\mathrm{C}(9)$ & $1.459(5)$ \\
$\mathrm{N}(3)-\mathrm{C}(2)$ & $1.409(5)$ \\
$\mathrm{N}(3)-\mathrm{C}(15)$ & $1.449(5)$ \\
$\mathrm{N}(3)-\mathrm{C}(20)$ & $1.459(5)$
\end{tabular}




\begin{tabular}{|c|c|}
\hline $\mathrm{N}(4)-\mathrm{C}(2)$ & $1.414(5)$ \\
\hline $\mathrm{N}(4)-\mathrm{C}(26)$ & $1.462(5)$ \\
\hline $\mathrm{N}(4)-\mathrm{C}(21)$ & $1.463(5)$ \\
\hline $\mathrm{C}(1)-\mathrm{C}(2)$ & $1.357(6)$ \\
\hline $\mathrm{C}(3)-\mathrm{C}(4)$ & $1.514(6)$ \\
\hline $\mathrm{C}(4)-\mathrm{C}(5)$ & $1.533(6)$ \\
\hline $\mathrm{C}(5)-\mathrm{C}(6)$ & $1.522(6)$ \\
\hline $\mathrm{C}(6)-\mathrm{C}(7)$ & $1.525(6)$ \\
\hline $\mathrm{C}(7)-\mathrm{C}(8)$ & $1.538(6)$ \\
\hline$C(9)-C(10)$ & $1.517(6)$ \\
\hline$C(10)-C(11)$ & $1.534(6)$ \\
\hline$C(11)-C(12)$ & $1.528(6)$ \\
\hline$C(12)-C(13)$ & $1.504(6)$ \\
\hline $\mathrm{C}(13)-\mathrm{C}(14)$ & $1.529(6)$ \\
\hline$C(15)-C(16)$ & $1.525(6)$ \\
\hline$C(16)-C(17)$ & $1.519(6)$ \\
\hline C(17)-C(18) & $1.523(7)$ \\
\hline C(18)-C(19) & $1.532(6)$ \\
\hline C(19)-C(20) & $1.502(6)$ \\
\hline$C(21)-C(22)$ & $1.518(6)$ \\
\hline$C(22)-C(23)$ & $1.535(6)$ \\
\hline $\mathrm{C}(23)-\mathrm{C}(24)$ & $1.510(6)$ \\
\hline$C(24)-C(25)$ & $1.516(6)$ \\
\hline$C(25)-C(26)$ & $1.522(6)$ \\
\hline $\mathrm{C}(1)-\mathrm{N}(1)-\mathrm{C}(8)$ & $120.1(3)$ \\
\hline $\mathrm{C}(1)-\mathrm{N}(1)-\mathrm{C}(3)$ & $117.4(3)$ \\
\hline $\mathrm{C}(8)-\mathrm{N}(1)-\mathrm{C}(3)$ & $121.2(3)$ \\
\hline $\mathrm{C}(1)-\mathrm{N}(2)-\mathrm{C}(14)$ & $120.3(3)$ \\
\hline $\mathrm{C}(1)-\mathrm{N}(2)-\mathrm{C}(9)$ & $116.7(3)$ \\
\hline $\mathrm{C}(14)-\mathrm{N}(2)-\mathrm{C}(9)$ & $122.4(3)$ \\
\hline $\mathrm{C}(2)-\mathrm{N}(3)-\mathrm{C}(15)$ & $117.7(3)$ \\
\hline $\mathrm{C}(2)-\mathrm{N}(3)-\mathrm{C}(20)$ & $120.4(3)$ \\
\hline $\mathrm{C}(15)-\mathrm{N}(3)-\mathrm{C}(20)$ & $120.4(3)$ \\
\hline $\mathrm{C}(2)-\mathrm{N}(4)-\mathrm{C}(26)$ & $117.2(3)$ \\
\hline $\mathrm{C}(2)-\mathrm{N}(4)-\mathrm{C}(21)$ & $118.5(3)$ \\
\hline $\mathrm{C}(26)-\mathrm{N}(4)-\mathrm{C}(21)$ & $118.2(3)$ \\
\hline $\mathrm{C}(2)-\mathrm{C}(1)-\mathrm{N}(2)$ & $122.4(4)$ \\
\hline $\mathrm{C}(2)-\mathrm{C}(1)-\mathrm{N}(1)$ & $123.4(4)$ \\
\hline $\mathrm{N}(2)-\mathrm{C}(1)-\mathrm{N}(1)$ & $114.2(3)$ \\
\hline $\mathrm{C}(1)-\mathrm{C}(2)-\mathrm{N}(3)$ & $123.3(4)$ \\
\hline $\mathrm{C}(1)-\mathrm{C}(2)-\mathrm{N}(4)$ & $123.5(4)$ \\
\hline $\mathrm{N}(3)-\mathrm{C}(2)-\mathrm{N}(4)$ & $113.2(3)$ \\
\hline $\mathrm{N}(1)-\mathrm{C}(3)-\mathrm{C}(4)$ & 115.1(3) \\
\hline $\mathrm{C}(3)-\mathrm{C}(4)-\mathrm{C}(5)$ & $114.0(3)$ \\
\hline$C(6)-C(5)-C(4)$ & $113.7(3)$ \\
\hline$C(5)-C(6)-C(7)$ & $115.0(3)$ \\
\hline$C(6)-C(7)-C(8)$ & $114.9(4)$ \\
\hline $\mathrm{N}(1)-\mathrm{C}(8)-\mathrm{C}(7)$ & $113.0(3)$ \\
\hline $\mathrm{N}(2)-\mathrm{C}(9)-\mathrm{C}(10)$ & $115.9(3)$ \\
\hline $\mathrm{C}(9)-\mathrm{C}(10)-\mathrm{C}(11)$ & $115.1(4)$ \\
\hline $\mathrm{C}(12)-\mathrm{C}(11)-\mathrm{C}(10)$ & $114.0(4)$ \\
\hline $\mathrm{C}(13)-\mathrm{C}(12)-\mathrm{C}(11)$ & $115.3(4)$ \\
\hline $\mathrm{C}(12)-\mathrm{C}(13)-\mathrm{C}(14)$ & $115.2(4)$ \\
\hline $\mathrm{N}(2)-\mathrm{C}(14)-\mathrm{C}(13)$ & $114.7(3)$ \\
\hline $\mathrm{N}(3)-\mathrm{C}(15)-\mathrm{C}(16)$ & $115.3(4)$ \\
\hline
\end{tabular}




$\begin{array}{ll}\mathrm{C}(17)-\mathrm{C}(16)-\mathrm{C}(15) & 114.7(4) \\ \mathrm{C}(16)-\mathrm{C}(17)-\mathrm{C}(18) & 115.1(4) \\ \mathrm{C}(17)-\mathrm{C}(18)-\mathrm{C}(19) & 114.8(4) \\ \mathrm{C}(20)-\mathrm{C}(19)-\mathrm{C}(18) & 114.6(4) \\ \mathrm{N}(3)-\mathrm{C}(20)-\mathrm{C}(19) & 117.2(4) \\ \mathrm{N}(4)-\mathrm{C}(21)-\mathrm{C}(22) & 114.1(3) \\ \mathrm{C}(21)-\mathrm{C}(22)-\mathrm{C}(23) & 115.5(4) \\ \mathrm{C}(24)-\mathrm{C}(23)-\mathrm{C}(22) & 115.3(3) \\ \mathrm{C}(23)-\mathrm{C}(24)-\mathrm{C}(25) & 113.3(4) \\ \mathrm{C}(24)-\mathrm{C}(25)-\mathrm{C}(26) & 116.2(4) \\ \mathrm{N}(4)-\mathrm{C}(26)-\mathrm{C}(25) & 114.3(3)\end{array}$

Table S25. Anisotropic displacement parameters $\left(\AA^{2} \times 10^{3}\right)$ for TAzE. The anisotropic displacement factor exponent takes the form: $-2 \pi^{2}\left[h^{2} a^{* 2} U^{11}+\ldots+2 h k a^{*} b^{*} U^{12}\right]$

\begin{tabular}{|c|c|c|c|c|c|c|}
\hline & $\mathrm{U}^{11}$ & $\mathrm{U}^{22}$ & $\mathrm{U}^{33}$ & $\mathrm{U}^{23}$ & $\mathrm{U}^{13}$ & $\mathrm{U}^{12}$ \\
\hline $\mathrm{N}(1)$ & $35(2)$ & 21(2) & $28(2)$ & $0(1)$ & $-1(1)$ & $-3(1)$ \\
\hline $\mathrm{N}(2)$ & $32(2)$ & $16(2)$ & $29(2)$ & $3(1)$ & $2(1)$ & $2(1)$ \\
\hline $\mathrm{N}(3)$ & $29(2)$ & $26(2)$ & $32(2)$ & $-5(2)$ & $4(1)$ & $-1(1)$ \\
\hline $\mathrm{N}(4)$ & $32(2)$ & $20(2)$ & $29(2)$ & $1(1)$ & $-1(1)$ & $2(1)$ \\
\hline $\mathrm{C}(1)$ & $34(2)$ & $13(2)$ & $33(2)$ & $0(2)$ & $2(2)$ & $2(2)$ \\
\hline $\mathrm{C}(2)$ & $33(2)$ & $15(2)$ & $31(2)$ & $-1(2)$ & $2(2)$ & $0(2)$ \\
\hline$C(3)$ & $37(2)$ & $22(2)$ & $35(2)$ & $0(2)$ & $-2(2)$ & $-5(2)$ \\
\hline $\mathrm{C}(4)$ & $37(2)$ & $25(2)$ & $32(2)$ & $-3(2)$ & $-1(2)$ & $-4(2)$ \\
\hline$C(5)$ & $34(2)$ & $32(2)$ & $34(2)$ & $0(2)$ & $-4(2)$ & $1(2)$ \\
\hline$C(6)$ & $39(2)$ & $30(2)$ & $32(2)$ & $6(2)$ & $0(2)$ & $5(2)$ \\
\hline$C(7)$ & $45(2)$ & $23(2)$ & $36(2)$ & $3(2)$ & $-1(2)$ & $-3(2)$ \\
\hline $\mathrm{C}(8)$ & $34(2)$ & $27(2)$ & $29(2)$ & $-1(2)$ & $-1(2)$ & $-5(2)$ \\
\hline $\mathrm{C}(9)$ & $34(2)$ & $22(2)$ & $35(2)$ & $4(2)$ & $-3(2)$ & $3(2)$ \\
\hline $\mathrm{C}(10)$ & $32(2)$ & $23(2)$ & $33(2)$ & $0(2)$ & $-3(2)$ & $3(2)$ \\
\hline$C(11)$ & $31(2)$ & $30(2)$ & $52(3)$ & $6(2)$ & $1(2)$ & $-2(2)$ \\
\hline $\mathrm{C}(12)$ & $37(2)$ & $28(2)$ & $42(2)$ & $8(2)$ & $4(2)$ & $-4(2)$ \\
\hline$C(13)$ & $41(2)$ & $19(2)$ & $38(2)$ & $2(2)$ & $1(2)$ & $-2(2)$ \\
\hline $\mathrm{C}(14)$ & $32(2)$ & $25(2)$ & $30(2)$ & $4(2)$ & $1(2)$ & $0(2)$ \\
\hline$C(15)$ & $39(2)$ & $22(2)$ & $33(2)$ & $-3(2)$ & $4(2)$ & $1(2)$ \\
\hline $\mathrm{C}(16)$ & 42(3) & $25(2)$ & $48(3)$ & $-6(2)$ & $3(2)$ & $2(2)$ \\
\hline $\mathrm{C}(17)$ & $47(3)$ & $32(2)$ & $46(3)$ & $-9(2)$ & $10(2)$ & $4(2)$ \\
\hline$C(18)$ & $43(3)$ & $36(3)$ & $43(3)$ & $-3(2)$ & $15(2)$ & $1(2)$ \\
\hline$C(19)$ & 48(3) & $26(2)$ & $47(3)$ & $-1(2)$ & $6(2)$ & $-3(2)$ \\
\hline $\mathrm{C}(20)$ & $36(2)$ & $29(2)$ & $39(2)$ & $-5(2)$ & $5(2)$ & $-5(2)$ \\
\hline $\mathrm{C}(21)$ & $38(2)$ & $21(2)$ & $33(2)$ & $2(2)$ & $-1(2)$ & $3(2)$ \\
\hline$C(22)$ & $36(2)$ & $32(2)$ & $31(2)$ & $2(2)$ & $-2(2)$ & $6(2)$ \\
\hline $\mathrm{C}(23)$ & $35(2)$ & $33(2)$ & $39(2)$ & $1(2)$ & $-9(2)$ & $-1(2)$ \\
\hline $\mathrm{C}(24)$ & 41(2) & $21(2)$ & $41(2)$ & $-1(2)$ & $-5(2)$ & $-4(2)$ \\
\hline $\mathrm{C}(25)$ & $42(2)$ & $20(2)$ & $35(2)$ & $-3(2)$ & $1(2)$ & $0(2)$ \\
\hline$C(26)$ & $34(2)$ & $19(2)$ & $33(2)$ & $-2(2)$ & $2(2)$ & $1(2)$ \\
\hline
\end{tabular}

Table S26. Torsion angles $\left[{ }^{\circ}\right]$ for TAzE.

\begin{tabular}{lr}
\hline $\mathrm{C}(14)-\mathrm{N}(2)-\mathrm{C}(1)-\mathrm{C}(2)$ & $-44.5(5)$ \\
$\mathrm{C}(9)-\mathrm{N}(2)-\mathrm{C}(1)-\mathrm{C}(2)$ & $126.8(4)$
\end{tabular}




\begin{tabular}{|c|c|}
\hline $\mathrm{C}(14)-\mathrm{N}(2)-\mathrm{C}(1)-\mathrm{N}(1)$ & $134.9(4)$ \\
\hline $\mathrm{C}(9)-\mathrm{N}(2)-\mathrm{C}(1)-\mathrm{N}(1)$ & $-53.7(4)$ \\
\hline $\mathrm{C}(8)-\mathrm{N}(1)-\mathrm{C}(1)-\mathrm{C}(2)$ & $-43.3(5)$ \\
\hline $\mathrm{C}(3)-\mathrm{N}(1)-\mathrm{C}(1)-\mathrm{C}(2)$ & $123.5(4)$ \\
\hline $\mathrm{C}(8)-\mathrm{N}(1)-\mathrm{C}(1)-\mathrm{N}(2)$ & $137.3(4)$ \\
\hline $\mathrm{C}(3)-\mathrm{N}(1)-\mathrm{C}(1)-\mathrm{N}(2)$ & $-56.0(5)$ \\
\hline $\mathrm{N}(2)-\mathrm{C}(1)-\mathrm{C}(2)-\mathrm{N}(3)$ & $149.3(4)$ \\
\hline $\mathrm{N}(1)-\mathrm{C}(1)-\mathrm{C}(2)-\mathrm{N}(3)$ & $-30.0(6)$ \\
\hline $\mathrm{N}(2)-\mathrm{C}(1)-\mathrm{C}(2)-\mathrm{N}(4)$ & $-29.0(6)$ \\
\hline $\mathrm{N}(1)-\mathrm{C}(1)-\mathrm{C}(2)-\mathrm{N}(4)$ & $151.6(4)$ \\
\hline $\mathrm{C}(15)-\mathrm{N}(3)-\mathrm{C}(2)-\mathrm{C}(1)$ & $-32.8(5)$ \\
\hline $\mathrm{C}(20)-\mathrm{N}(3)-\mathrm{C}(2)-\mathrm{C}(1)$ & $133.0(4)$ \\
\hline $\mathrm{C}(15)-\mathrm{N}(3)-\mathrm{C}(2)-\mathrm{N}(4)$ & $145.7(3)$ \\
\hline $\mathrm{C}(20)-\mathrm{N}(3)-\mathrm{C}(2)-\mathrm{N}(4)$ & $-48.5(5)$ \\
\hline $\mathrm{C}(26)-\mathrm{N}(4)-\mathrm{C}(2)-\mathrm{C}(1)$ & $-33.1(5)$ \\
\hline $\mathrm{C}(21)-\mathrm{N}(4)-\mathrm{C}(2)-\mathrm{C}(1)$ & $118.9(4)$ \\
\hline $\mathrm{C}(26)-\mathrm{N}(4)-\mathrm{C}(2)-\mathrm{N}(3)$ & $148.4(3)$ \\
\hline $\mathrm{C}(21)-\mathrm{N}(4)-\mathrm{C}(2)-\mathrm{N}(3)$ & $-59.6(5)$ \\
\hline $\mathrm{C}(1)-\mathrm{N}(1)-\mathrm{C}(3)-\mathrm{C}(4)$ & 174.1(4) \\
\hline $\mathrm{C}(8)-\mathrm{N}(1)-\mathrm{C}(3)-\mathrm{C}(4)$ & $-19.3(5)$ \\
\hline $\mathrm{N}(1)-\mathrm{C}(3)-\mathrm{C}(4)-\mathrm{C}(5)$ & $-57.0(5)$ \\
\hline $\mathrm{C}(3)-\mathrm{C}(4)-\mathrm{C}(5)-\mathrm{C}(6)$ & $87.4(5)$ \\
\hline$C(4)-C(5)-C(6)-C(7)$ & $-64.3(5)$ \\
\hline$C(5)-C(6)-C(7)-C(8)$ & $54.9(5)$ \\
\hline $\mathrm{C}(1)-\mathrm{N}(1)-\mathrm{C}(8)-\mathrm{C}(7)$ & $-113.7(4)$ \\
\hline $\mathrm{C}(3)-\mathrm{N}(1)-\mathrm{C}(8)-\mathrm{C}(7)$ & $80.1(5)$ \\
\hline $\mathrm{C}(6)-\mathrm{C}(7)-\mathrm{C}(8)-\mathrm{N}(1)$ & $-76.8(5)$ \\
\hline $\mathrm{C}(1)-\mathrm{N}(2)-\mathrm{C}(9)-\mathrm{C}(10)$ & $-179.5(3)$ \\
\hline $\mathrm{C}(14)-\mathrm{N}(2)-\mathrm{C}(9)-\mathrm{C}(10)$ & $-8.3(5)$ \\
\hline $\mathrm{N}(2)-\mathrm{C}(9)-\mathrm{C}(10)-\mathrm{C}(11)$ & $-61.6(5)$ \\
\hline $\mathrm{C}(9)-\mathrm{C}(10)-\mathrm{C}(11)-\mathrm{C}(12)$ & $83.9(5)$ \\
\hline $\mathrm{C}(10)-\mathrm{C}(11)-\mathrm{C}(12)-\mathrm{C}(13)$ & $-62.5(5)$ \\
\hline $\mathrm{C}(11)-\mathrm{C}(12)-\mathrm{C}(13)-\mathrm{C}(14)$ & $58.5(5)$ \\
\hline $\mathrm{C}(1)-\mathrm{N}(2)-\mathrm{C}(14)-\mathrm{C}(13)$ & $-118.0(4)$ \\
\hline $\mathrm{C}(9)-\mathrm{N}(2)-\mathrm{C}(14)-\mathrm{C}(13)$ & $71.2(5)$ \\
\hline $\mathrm{C}(12)-\mathrm{C}(13)-\mathrm{C}(14)-\mathrm{N}(2)$ & $-78.7(5)$ \\
\hline$C(2)-N(3)-C(15)-C(16)$ & $-160.0(4)$ \\
\hline $\mathrm{C}(20)-\mathrm{N}(3)-\mathrm{C}(15)-\mathrm{C}(16)$ & $34.3(5)$ \\
\hline $\mathrm{N}(3)-\mathrm{C}(15)-\mathrm{C}(16)-\mathrm{C}(17)$ & $-83.1(5)$ \\
\hline $\mathrm{C}(15)-\mathrm{C}(16)-\mathrm{C}(17)-\mathrm{C}(18)$ & $72.5(5)$ \\
\hline $\mathrm{C}(16)-\mathrm{C}(17)-\mathrm{C}(18)-\mathrm{C}(19)$ & $-54.1(6)$ \\
\hline $\mathrm{C}(17)-\mathrm{C}(18)-\mathrm{C}(19)-\mathrm{C}(20)$ & $67.9(6)$ \\
\hline $\mathrm{C}(2)-\mathrm{N}(3)-\mathrm{C}(20)-\mathrm{C}(19)$ & $-125.5(4)$ \\
\hline $\mathrm{C}(15)-\mathrm{N}(3)-\mathrm{C}(20)-\mathrm{C}(19)$ & $39.8(6)$ \\
\hline $\mathrm{C}(18)-\mathrm{C}(19)-\mathrm{C}(20)-\mathrm{N}(3)$ & $-83.3(5)$ \\
\hline$C(2)-N(4)-C(21)-C(22)$ & $120.4(4)$ \\
\hline $\mathrm{C}(26)-\mathrm{N}(4)-\mathrm{C}(21)-\mathrm{C}(22)$ & $-87.9(4)$ \\
\hline $\mathrm{N}(4)-\mathrm{C}(21)-\mathrm{C}(22)-\mathrm{C}(23)$ & $70.1(5)$ \\
\hline $\mathrm{C}(21)-\mathrm{C}(22)-\mathrm{C}(23)-\mathrm{C}(24)$ & $-50.5(5)$ \\
\hline $\mathrm{C}(22)-\mathrm{C}(23)-\mathrm{C}(24)-\mathrm{C}(25)$ & $67.5(5)$ \\
\hline$C(23)-C(24)-C(25)-C(26)$ & $-87.7(5)$ \\
\hline $\mathrm{C}(2)-\mathrm{N}(4)-\mathrm{C}(26)-\mathrm{C}(25)$ & $-170.9(3)$ \\
\hline $\mathrm{C}(21)-\mathrm{N}(4)-\mathrm{C}(26)-\mathrm{C}(25)$ & $37.0(5)$ \\
\hline $\mathrm{C}(24)-\mathrm{C}(25)-\mathrm{C}(26)-\mathrm{N}(4)$ & $42.8(5)$ \\
\hline
\end{tabular}


$X$-Ray Diffraction of [TPiE $]^{2+}$ [I]": Crystals suitable for X-ray diffraction were grown by slow vapor diffusion of $\mathrm{Et}_{2} \mathrm{O}$ into a nearly saturated solution of $\left[\mathrm{TPiE}^{2+}\right]\left[\mathrm{I}^{-}\right]_{2}$ in $\mathrm{CH}_{3} \mathrm{CN}$. Lowtemperature diffraction data ( $\omega$-scans) were collected on a Rigaku SCX Mini diffractometer coupled to a Rigaku Mercury275R CCD with Mo Ka radiation $(\lambda=0.71073 \AA)$ for the structure of $\left[\mathrm{TPiE}^{2+}\right]\left[\mathrm{I}^{-}\right]_{2}$. The diffraction images were processed and scaled using Rigaku Oxford Diffraction software. ${ }^{21}$ The structure was solved with SHELXT and was refined against $\mathrm{F}^{2}$ on all data by full-matrix least squares with SHELXL. ${ }^{22}$ All non-hydrogen atoms were refined anisotropically. Hydrogen atoms were included in the model at geometrically calculated positions and refined using a riding model. The isotropic displacement parameters of all hydrogen atoms were fixed to 1.2 times the $U$ value of the atoms to which they are linked (1.5 times for methyl groups). The full numbering scheme can be found in the full details of the X-ray structure determination (CIF), which is included as Supporting Information. CCDC number 2111621 contains the supplementary crystallographic data for this paper.

Table S27. Crystal data and structure refinement for [TPiE $]^{2+} 2[\mathrm{l}]-$.

\author{
Empirical formula \\ Formula weight \\ Temperature \\ Wavelength \\ Crystal system \\ Space group \\ Unit cell dimensions
}

Volume

Z

Density (calculated)

Absorption coefficient

$\mathrm{F}(000)$

Crystal size

Crystal color and habit

Diffractometer

Theta range for data collection

Index ranges

Reflections collected

Independent reflections

Observed reflections (I $>2$ sigma(I))

Completeness to theta $=25.242^{\circ}$

Absorption correction

Max. and min. transmission

Solution method

Refinement method

Data / restraints / parameters

Goodness-of-fit on $\mathrm{F}^{2}$

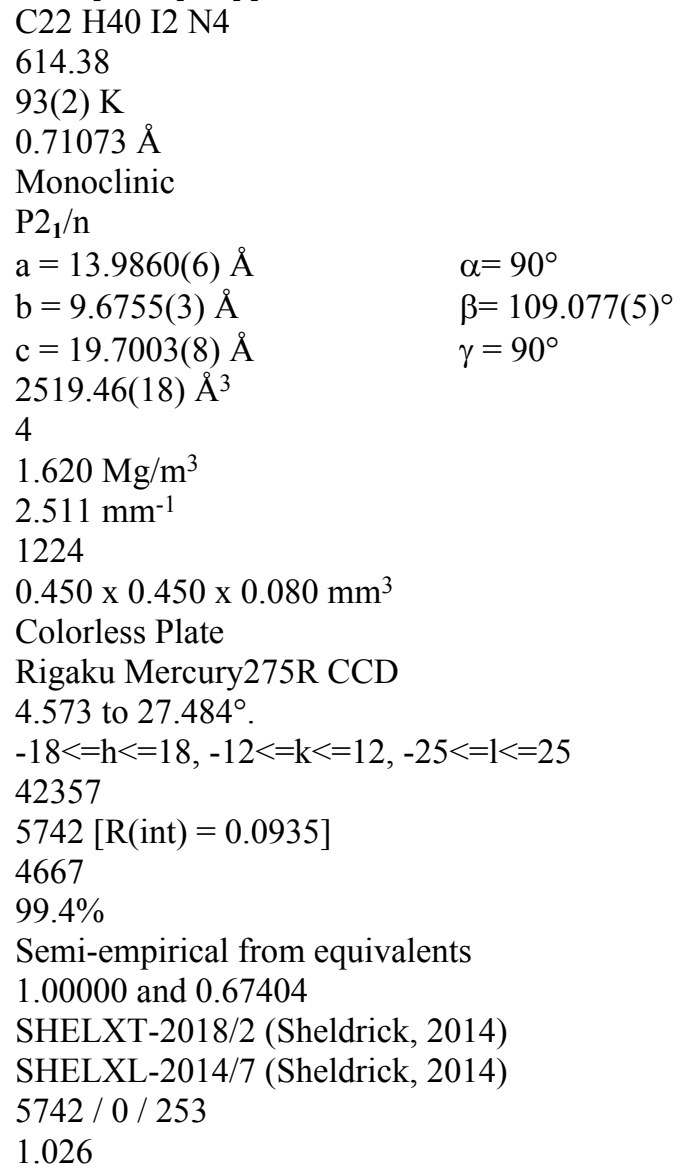


Final $\mathrm{R}$ indices $[\mathrm{I}>2 \operatorname{sigma}(\mathrm{I})]$

$\mathrm{R}$ indices (all data)

Extinction coefficient

Largest diff. peak and hole
$\mathrm{R} 1=0.0348, \mathrm{wR} 2=0.0741$

$\mathrm{R} 1=0.0501, \mathrm{wR} 2=0.0798$

$\mathrm{n} / \mathrm{a}$

1.110 and -0.651 e. $\AA^{-3}$

Table S28. Atomic coordinates $\left(\times 10^{4}\right)$ and equivalent isotropic displacement parameters $\left(\AA^{2} \times 10^{3}\right)$ for $[\mathrm{TPiE}]^{2+} 2[\mathrm{I}] \cdot \mathrm{U}(\mathrm{eq})$ is defined as one third of the trace of the orthogonalized $\mathrm{Uij}$ tensor.

\begin{tabular}{|c|c|c|c|c|}
\hline & $\mathrm{x}$ & $\mathrm{y}$ & $\mathrm{Z}$ & $\mathrm{U}(\mathrm{eq})$ \\
\hline $\mathrm{I}(1)$ & $1492(1)$ & $7516(1)$ & $1857(1)$ & $21(1)$ \\
\hline $\mathrm{I}(2)$ & $7424(1)$ & $9893(1)$ & $3354(1)$ & $19(1)$ \\
\hline $\mathrm{N}(1)$ & $4909(2)$ & $7975(3)$ & $2096(2)$ & $13(1)$ \\
\hline $\mathrm{N}(2)$ & $4570(2)$ & $7383(3)$ & $3151(2)$ & $12(1)$ \\
\hline $\mathrm{N}(3)$ & $4520(2)$ & $4880(3)$ & $1936(2)$ & $12(1)$ \\
\hline $\mathrm{N}(4)$ & $5952(2)$ & $5110(3)$ & $2975(2)$ & $12(1)$ \\
\hline $\mathrm{C}(1)$ & $4836(2)$ & $7078(3)$ & $2582(2)$ & $12(1)$ \\
\hline $\mathrm{C}(2)$ & $5114(2)$ & $5576(3)$ & $2494(2)$ & $12(1)$ \\
\hline $\mathrm{C}(3)$ & $4389(3)$ & $9326(3)$ & $1969(2)$ & $17(1)$ \\
\hline $\mathrm{C}(4)$ & $3890(3)$ & $9556(4)$ & $1163(2)$ & $19(1)$ \\
\hline$C(5)$ & $4646(3)$ & $9420(4)$ & $758(2)$ & $19(1)$ \\
\hline $\mathrm{C}(6)$ & $5121(3)$ & $7988(4)$ & $893(2)$ & $18(1)$ \\
\hline$C(7)$ & $5630(3)$ & $7747(4)$ & $1692(2)$ & $16(1)$ \\
\hline $\mathrm{C}(8)$ & $4672(3)$ & $8792(3)$ & $3472(2)$ & $17(1)$ \\
\hline $\mathrm{C}(9)$ & $5132(3)$ & $8685(4)$ & $4284(2)$ & $18(1)$ \\
\hline$C(10)$ & $4528(3)$ & $7734(4)$ & $4608(2)$ & $20(1)$ \\
\hline $\mathrm{C}(11)$ & $4421(3)$ & $6310(4)$ & $4264(2)$ & $16(1)$ \\
\hline$C(12)$ & $4009(3)$ & $6373(4)$ & $3451(2)$ & $16(1)$ \\
\hline$C(13)$ & $3418(2)$ & $5179(4)$ & $1642(2)$ & $16(1)$ \\
\hline$C(14)$ & $3020(3)$ & $5177(4)$ & $828(2)$ & $18(1)$ \\
\hline$C(15)$ & $3329(3)$ & $3867(4)$ & $523(2)$ & $22(1)$ \\
\hline$C(16)$ & $4474(3)$ & $3696(4)$ & $823(2)$ & $19(1)$ \\
\hline$C(17)$ & $4849(3)$ & $3631(3)$ & $1639(2)$ & $15(1)$ \\
\hline $\mathrm{C}(18)$ & $6804(3)$ & $6049(3)$ & $3339(2)$ & $16(1)$ \\
\hline C(19) & $7165(3)$ & $5857(4)$ & $4149(2)$ & $19(1)$ \\
\hline $\mathrm{C}(20)$ & $7383(3)$ & $4344(4)$ & $4352(2)$ & 21(1) \\
\hline $\mathrm{C}(21)$ & $6475(3)$ & $3450(4)$ & $3962(2)$ & $17(1)$ \\
\hline$C(22)$ & $6165(3)$ & $3623(3)$ & $3152(2)$ & $14(1)$ \\
\hline
\end{tabular}

Table S29. Bond lengths $[\AA]$ and angles $\left[{ }^{\circ}\right]$ for $[\mathrm{TPiE}]^{2+} 2[\mathrm{I}]$.

\begin{tabular}{ll}
\hline $\mathrm{N}(1)-\mathrm{C}(1)$ & $1.320(4)$ \\
$\mathrm{N}(1)-\mathrm{C}(3)$ & $1.477(4)$ \\
$\mathrm{N}(1)-\mathrm{C}(7)$ & $1.491(4)$ \\
$\mathrm{N}(2)-\mathrm{C}(1)$ & $1.325(4)$ \\
$\mathrm{N}(2)-\mathrm{C}(8)$ & $1.490(4)$ \\
$\mathrm{N}(2)-\mathrm{C}(12)$ & $1.490(4)$ \\
$\mathrm{N}(3)-\mathrm{C}(2)$ & $1.325(4)$ \\
$\mathrm{N}(3)-\mathrm{C}(17)$ & $1.480(4)$ \\
$\mathrm{N}(3)-\mathrm{C}(13)$ & $1.486(4)$ \\
$\mathrm{N}(4)-\mathrm{C}(2)$ & $1.322(4)$ \\
$\mathrm{N}(4)-\mathrm{C}(18)$ & $1.483(4)$ \\
$\mathrm{N}(4)-\mathrm{C}(22)$ & $1.487(4)$
\end{tabular}




\begin{tabular}{|c|c|}
\hline$C(1)-C(2)$ & $1.530(4)$ \\
\hline$C(3)-C(4)$ & $1.527(5)$ \\
\hline$C(4)-C(5)$ & $1.524(5)$ \\
\hline $\mathrm{C}(5)-\mathrm{C}(6)$ & $1.522(5)$ \\
\hline$C(6)-C(7)$ & $1.519(5)$ \\
\hline $\mathrm{C}(8)-\mathrm{C}(9)$ & $1.520(5)$ \\
\hline $\mathrm{C}(9)-\mathrm{C}(10)$ & $1.524(5)$ \\
\hline $\mathrm{C}(10)-\mathrm{C}(11)$ & $1.521(5)$ \\
\hline$C(11)-C(12)$ & $1.517(5)$ \\
\hline C(13)-C(14) & $1.516(5)$ \\
\hline$C(14)-C(15)$ & $1.525(5)$ \\
\hline$C(15)-C(16)$ & $1.525(5)$ \\
\hline$C(16)-C(17)$ & $1.521(5)$ \\
\hline $\mathrm{C}(18)-\mathrm{C}(19)$ & $1.520(5)$ \\
\hline C(19)-C(20) & $1.522(5)$ \\
\hline$C(20)-C(21)$ & $1.521(5)$ \\
\hline$C(21)-C(22)$ & $1.520(5)$ \\
\hline $\mathrm{C}(1)-\mathrm{N}(1)-\mathrm{C}(3)$ & $123.7(3)$ \\
\hline $\mathrm{C}(1)-\mathrm{N}(1)-\mathrm{C}(7)$ & $121.0(3)$ \\
\hline $\mathrm{C}(3)-\mathrm{N}(1)-\mathrm{C}(7)$ & $115.0(3)$ \\
\hline $\mathrm{C}(1)-\mathrm{N}(2)-\mathrm{C}(8)$ & $123.2(3)$ \\
\hline $\mathrm{C}(1)-\mathrm{N}(2)-\mathrm{C}(12)$ & $121.3(3)$ \\
\hline $\mathrm{C}(8)-\mathrm{N}(2)-\mathrm{C}(12)$ & $115.0(3)$ \\
\hline $\mathrm{C}(2)-\mathrm{N}(3)-\mathrm{C}(17)$ & $123.4(3)$ \\
\hline $\mathrm{C}(2)-\mathrm{N}(3)-\mathrm{C}(13)$ & $120.9(3)$ \\
\hline $\mathrm{C}(17)-\mathrm{N}(3)-\mathrm{C}(13)$ & $115.0(3)$ \\
\hline $\mathrm{C}(2)-\mathrm{N}(4)-\mathrm{C}(18)$ & $121.4(3)$ \\
\hline $\mathrm{C}(2)-\mathrm{N}(4)-\mathrm{C}(22)$ & $124.0(3)$ \\
\hline $\mathrm{C}(18)-\mathrm{N}(4)-\mathrm{C}(22)$ & $114.3(3)$ \\
\hline $\mathrm{N}(1)-\mathrm{C}(1)-\mathrm{N}(2)$ & $125.2(3)$ \\
\hline $\mathrm{N}(1)-\mathrm{C}(1)-\mathrm{C}(2)$ & $117.4(3)$ \\
\hline $\mathrm{N}(2)-\mathrm{C}(1)-\mathrm{C}(2)$ & $117.3(3)$ \\
\hline $\mathrm{N}(4)-\mathrm{C}(2)-\mathrm{N}(3)$ & $126.2(3)$ \\
\hline $\mathrm{N}(4)-\mathrm{C}(2)-\mathrm{C}(1)$ & $116.2(3)$ \\
\hline $\mathrm{N}(3)-\mathrm{C}(2)-\mathrm{C}(1)$ & $117.6(3)$ \\
\hline $\mathrm{N}(1)-\mathrm{C}(3)-\mathrm{C}(4)$ & $109.8(3)$ \\
\hline$C(5)-C(4)-C(3)$ & $111.8(3)$ \\
\hline$C(6)-C(5)-C(4)$ & $109.0(3)$ \\
\hline$C(7)-C(6)-C(5)$ & $110.6(3)$ \\
\hline $\mathrm{N}(1)-\mathrm{C}(7)-\mathrm{C}(6)$ & 111.1(3) \\
\hline $\mathrm{N}(2)-\mathrm{C}(8)-\mathrm{C}(9)$ & $109.5(3)$ \\
\hline C(8)-C(9)-C(10) & $112.3(3)$ \\
\hline $\mathrm{C}(11)-\mathrm{C}(10)-\mathrm{C}(9)$ & $110.3(3)$ \\
\hline $\mathrm{C}(12)-\mathrm{C}(11)-\mathrm{C}(10)$ & $112.5(3)$ \\
\hline $\mathrm{N}(2)-\mathrm{C}(12)-\mathrm{C}(11)$ & $112.2(3)$ \\
\hline N(3)-C(13)-C(14) & $112.8(3)$ \\
\hline $\mathrm{C}(13)-\mathrm{C}(14)-\mathrm{C}(15)$ & $111.4(3)$ \\
\hline $\mathrm{C}(16)-\mathrm{C}(15)-\mathrm{C}(14)$ & $109.5(3)$ \\
\hline$C(17)-C(16)-C(15)$ & $111.7(3)$ \\
\hline $\mathrm{N}(3)-\mathrm{C}(17)-\mathrm{C}(16)$ & 109.9(3) \\
\hline $\mathrm{N}(4)-\mathrm{C}(18)-\mathrm{C}(19)$ & $111.8(3)$ \\
\hline $\mathrm{C}(18)-\mathrm{C}(19)-\mathrm{C}(20)$ & $111.3(3)$ \\
\hline$C(21)-C(20)-C(19)$ & $110.6(3)$ \\
\hline$C(22)-C(21)-C(20)$ & $111.9(3)$ \\
\hline $\mathrm{N}(4)-\mathrm{C}(22)-\mathrm{C}(21)$ & $108.6(3)$ \\
\hline
\end{tabular}


Symmetry transformations used to generate equivalent atoms:

Table S30. Anisotropic displacement parameters $\left(\AA^{2} \times 10^{3}\right)$ for [TPiE $]^{2+} 2[\mathrm{l}]$ - The anisotropic displacement factor exponent takes the form: $-2 \pi^{2}\left[\mathrm{~h}^{2} \mathrm{a}^{* 2} \mathrm{U}^{11}+\ldots+2 \mathrm{~h} \mathrm{k} \mathrm{a}^{*} \mathrm{~b}^{*} \mathrm{U}^{12}\right]$

\begin{tabular}{lcccccc}
\hline & $\mathrm{U}^{11}$ & $\mathrm{U}^{22}$ & $\mathrm{U}^{33}$ & $\mathrm{U}^{23}$ & $\mathrm{U}^{13}$ & $\mathrm{U}^{12}$ \\
\hline $\mathrm{I}(1)$ & $18(1)$ & $16(1)$ & $28(1)$ & $-3(1)$ & $7(1)$ & $4(1)$ \\
$\mathrm{I}(2)$ & $21(1)$ & $16(1)$ & $24(1)$ & $-4(1)$ & $11(1)$ & $-5(1)$ \\
$\mathrm{N}(1)$ & $16(1)$ & $13(1)$ & $11(1)$ & $1(1)$ & $6(1)$ & $5(1)$ \\
$\mathrm{N}(2)$ & $15(1)$ & $10(1)$ & $11(1)$ & $2(1)$ & $3(1)$ & $1(1)$ \\
$\mathrm{N}(3)$ & $11(1)$ & $13(1)$ & $13(1)$ & $-1(1)$ & $3(1)$ & $1(1)$ \\
$\mathrm{N}(4)$ & $14(1)$ & $9(1)$ & $11(1)$ & $0(1)$ & $2(1)$ & $-2(1)$ \\
$\mathrm{C}(1)$ & $9(2)$ & $12(2)$ & $12(2)$ & $-1(1)$ & $-1(1)$ & $0(1)$ \\
$\mathrm{C}(2)$ & $13(2)$ & $14(2)$ & $10(2)$ & $1(1)$ & $7(1)$ & $-2(1)$ \\
$\mathrm{C}(3)$ & $22(2)$ & $11(2)$ & $18(2)$ & $5(1)$ & $7(2)$ & $6(1)$ \\
$\mathrm{C}(4)$ & $20(2)$ & $21(2)$ & $17(2)$ & $7(2)$ & $6(2)$ & $2(1)$ \\
$\mathrm{C}(5)$ & $18(2)$ & $25(2)$ & $13(2)$ & $5(2)$ & $2(2)$ & $-4(2)$ \\
$\mathrm{C}(6)$ & $18(2)$ & $22(2)$ & $15(2)$ & $-2(1)$ & $6(2)$ & $-6(2)$ \\
$\mathrm{C}(7)$ & $16(2)$ & $20(2)$ & $14(2)$ & $0(1)$ & $8(1)$ & $0(1)$ \\
$\mathrm{C}(8)$ & $30(2)$ & $9(2)$ & $13(2)$ & $-3(1)$ & $10(2)$ & $0(1)$ \\
$\mathrm{C}(9)$ & $26(2)$ & $15(2)$ & $15(2)$ & $-2(1)$ & $8(2)$ & $1(1)$ \\
$\mathrm{C}(10)$ & $26(2)$ & $22(2)$ & $17(2)$ & $0(2)$ & $13(2)$ & $1(2)$ \\
$\mathrm{C}(11)$ & $18(2)$ & $19(2)$ & $13(2)$ & $3(1)$ & $6(1)$ & $2(1)$ \\
$\mathrm{C}(12)$ & $19(2)$ & $15(2)$ & $15(2)$ & $1(1)$ & $7(2)$ & $-1(1)$ \\
$\mathrm{C}(13)$ & $12(2)$ & $17(2)$ & $16(2)$ & $-4(1)$ & $0(1)$ & $0(1)$ \\
$\mathrm{C}(14)$ & $16(2)$ & $20(2)$ & $16(2)$ & $2(2)$ & $3(1)$ & $-2(1)$ \\
$\mathrm{C}(15)$ & $26(2)$ & $24(2)$ & $13(2)$ & $-2(2)$ & $0(2)$ & $-2(2)$ \\
$\mathrm{C}(16)$ & $25(2)$ & $18(2)$ & $15(2)$ & $-6(1)$ & $6(2)$ & $1(2)$ \\
$\mathrm{C}(17)$ & $16(2)$ & $12(2)$ & $17(2)$ & $-3(1)$ & $6(1)$ & $2(1)$ \\
$\mathrm{C}(18)$ & $14(2)$ & $11(2)$ & $19(2)$ & $1(1)$ & $2(1)$ & $-1(1)$ \\
$\mathrm{C}(19)$ & $15(2)$ & $18(2)$ & $19(2)$ & $-3(2)$ & $-1(2)$ & $-2(1)$ \\
$\mathrm{C}(20)$ & $20(2)$ & $21(2)$ & $15(2)$ & $3(2)$ & $-3(2)$ & $4(2)$ \\
$\mathrm{C}(21)$ & $23(2)$ & $13(2)$ & $14(2)$ & $1(1)$ & $4(2)$ & $2(1)$ \\
$\mathrm{C}(22)$ & $13(2)$ & $10(2)$ & $18(2)$ & $1(1)$ & $3(1)$ & $2(1)$ \\
& & & & & & \\
\hline & & & & & & \\
\end{tabular}

Table S31. Torsion angles $\left[^{\circ}\right]$ for $[\mathrm{TPiE}]^{2+} 2[\mathrm{I}]$.

\begin{tabular}{ll}
\hline $\mathrm{C}(3)-\mathrm{N}(1)-\mathrm{C}(1)-\mathrm{N}(2)$ & $-21.8(5)$ \\
$\mathrm{C}(7)-\mathrm{N}(1)-\mathrm{C}(1)-\mathrm{N}(2)$ & $151.1(3)$ \\
$\mathrm{C}(3)-\mathrm{N}(1)-\mathrm{C}(1)-\mathrm{C}(2)$ & $160.5(3)$ \\
$\mathrm{C}(7)-\mathrm{N}(1)-\mathrm{C}(1)-\mathrm{C}(2)$ & $-26.6(4)$ \\
$\mathrm{C}(8)-\mathrm{N}(2)-\mathrm{C}(1)-\mathrm{N}(1)$ & $-22.8(5)$ \\
$\mathrm{C}(12)-\mathrm{N}(2)-\mathrm{C}(1)-\mathrm{N}(1)$ & $148.3(3)$ \\
$\mathrm{C}(8)-\mathrm{N}(2)-\mathrm{C}(1)-\mathrm{C}(2)$ & $155.0(3)$ \\
$\mathrm{C}(12)-\mathrm{N}(2)-\mathrm{C}(1)-\mathrm{C}(2)$ & $-33.9(4)$ \\
$\mathrm{C}(18)-\mathrm{N}(4)-\mathrm{C}(2)-\mathrm{N}(3)$ & $149.1(3)$ \\
$\mathrm{C}(22)-\mathrm{N}(4)-\mathrm{C}(2)-\mathrm{N}(3)$ & $-25.2(5)$ \\
$\mathrm{C}(18)-\mathrm{N}(4)-\mathrm{C}(2)-\mathrm{C}(1)$ & $-28.5(4)$ \\
$\mathrm{C}(22)-\mathrm{N}(4)-\mathrm{C}(2)-\mathrm{C}(1)$ & $157.1(3)$ \\
$\mathrm{C}(17)-\mathrm{N}(3)-\mathrm{C}(2)-\mathrm{N}(4)$ & $-18.9(5)$ \\
$\mathrm{C}(13)-\mathrm{N}(3)-\mathrm{C}(2)-\mathrm{N}(4)$ & $150.9(3)$
\end{tabular}




$\begin{array}{lc}\mathrm{C}(17)-\mathrm{N}(3)-\mathrm{C}(2)-\mathrm{C}(1) & \\ \mathrm{C}(13)-\mathrm{N}(3)-\mathrm{C}(2)-\mathrm{C}(1) & 158.7(3) \\ \mathrm{N}(1)-\mathrm{C}(1)-\mathrm{C}(2)-\mathrm{N}(4) & -31.5(4) \\ \mathrm{N}(2)-\mathrm{C}(1)-\mathrm{C}(2)-\mathrm{N}(4) & 110.6(3) \\ \mathrm{N}(1)-\mathrm{C}(1)-\mathrm{C}(2)-\mathrm{N}(3) & -67.4(4) \\ \mathrm{N}(2)-\mathrm{C}(1)-\mathrm{C}(2)-\mathrm{N}(3) & -67.3(4) \\ \mathrm{C}(1)-\mathrm{N}(1)-\mathrm{C}(3)-\mathrm{C}(4) & 114.7(3) \\ \mathrm{C}(7)-\mathrm{N}(1)-\mathrm{C}(3)-\mathrm{C}(4) & -134.0(3) \\ \mathrm{N}(1)-\mathrm{C}(3)-\mathrm{C}(4)-\mathrm{C}(5) & 52.7(4) \\ \mathrm{C}(3)-\mathrm{C}(4)-\mathrm{C}(5)-\mathrm{C}(6) & -55.0(4) \\ \mathrm{C}(4)-\mathrm{C}(5)-\mathrm{C}(6)-\mathrm{C}(7) & 58.4(4) \\ \mathrm{C}(1)-\mathrm{N}(1)-\mathrm{C}(7)-\mathrm{C}(6) & -57.7(4) \\ \mathrm{C}(3)-\mathrm{N}(1)-\mathrm{C}(7)-\mathrm{C}(6) & 133.0(3) \\ \mathrm{C}(5)-\mathrm{C}(6)-\mathrm{C}(7)-\mathrm{N}(1) & -53.5(4) \\ \mathrm{C}(1)-\mathrm{N}(2)-\mathrm{C}(8)-\mathrm{C}(9) & 55.0(4) \\ \mathrm{C}(12)-\mathrm{N}(2)-\mathrm{C}(8)-\mathrm{C}(9) & -134.6(3) \\ \mathrm{N}(2)-\mathrm{C}(8)-\mathrm{C}(9)-\mathrm{C}(10) & 53.8(4) \\ \mathrm{C}(8)-\mathrm{C}(9)-\mathrm{C}(10)-\mathrm{C}(11) & -55.8(4) \\ \mathrm{C}(9)-\mathrm{C}(10)-\mathrm{C}(11)-\mathrm{C}(12) & 56.0(4) \\ \mathrm{C}(1)-\mathrm{N}(2)-\mathrm{C}(12)-\mathrm{C}(11) & -52.4(4) \\ \mathrm{C}(8)-\mathrm{N}(2)-\mathrm{C}(12)-\mathrm{C}(11) & 136.6(3) \\ \mathrm{C}(10)-\mathrm{C}(11)-\mathrm{C}(12)-\mathrm{N}(2) & -51.6(4) \\ \mathrm{C}(2)-\mathrm{N}(3)-\mathrm{C}(13)-\mathrm{C}(14) & 50.0(4) \\ \mathrm{C}(17)-\mathrm{N}(3)-\mathrm{C}(13)-\mathrm{C}(14) & 138.6(3) \\ \mathrm{N}(3)-\mathrm{C}(13)-\mathrm{C}(14)-\mathrm{C}(15) & -50.8(4) \\ \mathrm{C}(13)-\mathrm{C}(14)-\mathrm{C}(15)-\mathrm{C}(16) & 51.0(4) \\ \mathrm{C}(14)-\mathrm{C}(15)-\mathrm{C}(16)-\mathrm{C}(17) & -54.6(4) \\ \mathrm{C}(2)-\mathrm{N}(3)-\mathrm{C}(17)-\mathrm{C}(16) & 58.1(4) \\ \mathrm{C}(13)-\mathrm{N}(3)-\mathrm{C}(17)-\mathrm{C}(16) & -137.1(3) \\ \mathrm{C}(15)-\mathrm{C}(16)-\mathrm{C}(17)-\mathrm{N}(3) & 52.5(4) \\ \mathrm{C}(2)-\mathrm{N}(4)-\mathrm{C}(18)-\mathrm{C}(19) & -56.3(4) \\ \mathrm{C}(22)-\mathrm{N}(4)-\mathrm{C}(18)-\mathrm{C}(19) & 130.4(3) \\ \mathrm{N}(4)-\mathrm{C}(18)-\mathrm{C}(19)-\mathrm{C}(20) & -54.7(4) \\ \mathrm{C}(18)-\mathrm{C}(19)-\mathrm{C}(20)-\mathrm{C}(21) & 51.7(4) \\ \mathrm{C}(19)-\mathrm{C}(20)-\mathrm{C}(21)-\mathrm{C}(22) & -53.0(4) \\ \mathrm{C}(2)-\mathrm{N}(4)-\mathrm{C}(22)-\mathrm{C}(21) & 56.6(4) \\ \mathrm{C}(18)-\mathrm{N}(4)-\mathrm{C}(22)-\mathrm{C}(21) & -129.0(3) \\ \mathrm{C}(20)-\mathrm{C}(21)-\mathrm{C}(22)-\mathrm{N}(4) & 56.3(4) \\ & -56.8(4) \\ & \\ & \end{array}$

Symmetry transformations used to generate equivalent atoms: 
$X$-Ray Diffraction of $[T P y E]^{2+} 2[I] \because$ Crystals suitable for X-ray diffraction were grown by slow vapor diffusion of $\mathrm{Et}_{2} \mathrm{O}$ into a solution of $\left[\mathrm{TPyE}^{2+}\right]\left[\mathrm{I}^{-}\right]_{2}$ in methanol. Low-temperature diffraction data ( $\omega$-scans) were collected on a Rigaku MicroMax-007HF diffractometer coupled to a Dectris Pilatus3R detector with Mo K $\alpha(\lambda=0.71073 \AA)$ for the structure of $\left[\mathrm{TPyE}^{2+}\right]\left[\mathrm{I}^{-}\right]_{2}$. The diffraction images were processed and scaled using Rigaku Oxford Diffraction software. ${ }^{21}$ The structure was solved with SHELXT and was refined against $\mathrm{F}^{2}$ on all data by full-matrix least squares with SHELXL. ${ }^{22}$ All non-hydrogen atoms were refined anisotropically. Hydrogen atoms were included in the model at geometrically calculated positions and refined using a riding model. The isotropic displacement parameters of all hydrogen atoms were fixed to 1.2 times the $U$ value of the atoms to which they are linked (1.5 times for methyl groups). The program SQUEEZE ${ }^{23}$ was used to compensate for the contribution of disordered solvents contained in voids within the crystal lattice from the diffraction intensities. This procedure was applied to the data file and the submitted model is based on the solvent removed data. Based on the total electron density found in the voids (158 $\left.\mathrm{e} / \mathrm{A}^{\wedge} 3^{\wedge}\right)$, it is likely that $\sim 8.5$ methanols, $\sim 4$ ethers, or some combination of these solvent molecules are present in the unit cell. See "_platon_squeeze_details" in the .cif for more information. The full numbering scheme can be found in the full details of the X-ray structure determination (CIF), which is included as Supporting Information. CCDC number 2111620 contains the supplementary crystallographic data for this paper.

Table S32. Crystal data and structure refinement for [TPyE $]^{2+} 2[1]$.

Empirical formula

Formula weight

Temperature

Wavelength

Crystal system

Space group

Unit cell dimensions

Volume

Z

Density (calculated)

Absorption coefficient

$\mathrm{F}(000)$

Crystal size

Crystal color and habit

Diffractometer

Theta range for data collection

Index ranges

Reflections collected

Independent reflections
C18 H32 I2 N4

558.27

93(2) $\mathrm{K}$

$0.71073 \AA$

Monoclinic

$\mathrm{C} 2 / \mathrm{c}$

$\mathrm{a}=14.2990(4) \AA$

$\mathrm{b}=13.0573(3) \AA$

$\mathrm{c}=14.8142(4) \AA$

2509.83(13) $\AA^{3}$

4

$1.477 \mathrm{Mg} / \mathrm{m}^{3}$

$2.513 \mathrm{~mm}^{-1}$

1096

$0.100 \times 0.100 \times 0.070 \mathrm{~mm}^{3}$

Colorless Block

Dectris Pilatus 3R

2.279 to $30.504^{\circ}$.

$-20<=\mathrm{h}<=20,-18<=\mathrm{k}<=18,-20<=\mathrm{l}<=21$

48356

$3719[\mathrm{R}(\mathrm{int})=0.0419]$ 
Observed reflections (I $>2$ sigma(I))

Completeness to theta $=25.242^{\circ}$

Absorption correction

Max. and min. transmission

Solution method

Refinement method

Data / restraints / parameters

Goodness-of-fit on $\mathrm{F}^{2}$

Final $R$ indices [I $>2 \operatorname{sigma}(\mathrm{I})]$

$\mathrm{R}$ indices (all data)

Extinction coefficient

Largest diff. peak and hole
3390

$100.0 \%$

Semi-empirical from equivalents

1.00000 and 0.75624

SHELXT-2014/5 (Sheldrick, 2014)

SHELXL-2014/7 (Sheldrick, 2014)

3719 / 0 / 110

1.268

$\mathrm{R} 1=0.0571, \mathrm{wR} 2=0.1374$

$\mathrm{R} 1=0.0619, \mathrm{wR} 2=0.1386$

$\mathrm{n} / \mathrm{a}$

3.198 and -1.548 e. $\AA^{-3}$

Table S33. Atomic coordinates $\left(\times 10^{4}\right)$ and equivalent isotropic displacement parameters $\left(\AA^{2} \times 10^{3}\right)$ for $[T P y E]^{2+} 2[\mathrm{I}]-\mathrm{U}(\mathrm{eq})$ is defined as one third of the trace of the orthogonalized $\mathrm{Uij}$ tensor.

\begin{tabular}{|c|c|c|c|c|}
\hline & $\mathrm{x}$ & $\mathrm{y}$ & $\mathrm{z}$ & $\mathrm{U}(\mathrm{eq})$ \\
\hline $\mathrm{I}(1)$ & $7023(1)$ & $8637(1)$ & $11529(1)$ & $27(1)$ \\
\hline $\mathrm{N}(1)$ & $5169(3)$ & $7638(3)$ & $6783(3)$ & $14(1)$ \\
\hline $\mathrm{N}(2)$ & $5833(3)$ & $9686(3)$ & $8153(3)$ & $13(1)$ \\
\hline $\mathrm{C}(1)$ & 5000 & $8078(5)$ & 7500 & $14(1)$ \\
\hline $\mathrm{C}(2)$ & 5000 & $9245(5)$ & 7500 & $14(1)$ \\
\hline $\mathrm{C}(3)$ & 4999(4) & $6543(4)$ & $6493(4)$ & 19(1) \\
\hline $\mathrm{C}(4)$ & $4975(4)$ & $6555(4)$ & $5453(4)$ & $20(1)$ \\
\hline$C(5)$ & $5744(4)$ & $7389(4)$ & $5528(4)$ & $23(1)$ \\
\hline $\mathrm{C}(6)$ & $5491(4)$ & $8214(4)$ & $6098(4)$ & $18(1)$ \\
\hline$C(7)$ & $6805(4)$ & $9115(4)$ & $8744(4)$ & $18(1)$ \\
\hline $\mathrm{C}(8)$ & $7559(4)$ & $9969(4)$ & $9286(4)$ & 19(1) \\
\hline $\mathrm{C}(9)$ & $6868(4)$ & $10765(4)$ & $9464(4)$ & $18(1)$ \\
\hline$C(10)$ & $5904(4)$ & $10775(4)$ & $8479(4)$ & $17(1)$ \\
\hline
\end{tabular}

Table S34. Bond lengths $[\AA]$ and angles $\left[{ }^{\circ}\right]$ for $[\mathrm{TPyE}]^{2+} 2[\mathrm{l}]$.

\begin{tabular}{ll}
\hline $\mathrm{N}(1)-\mathrm{C}(1)$ & $1.316(5)$ \\
$\mathrm{N}(1)-\mathrm{C}(3)$ & $1.482(6)$ \\
$\mathrm{N}(1)-\mathrm{C}(6)$ & $1.483(6)$ \\
$\mathrm{N}(2)-\mathrm{C}(2)$ & $1.311(5)$ \\
$\mathrm{N}(2)-\mathrm{C}(10)$ & $1.492(6)$ \\
$\mathrm{N}(2)-\mathrm{C}(7)$ & $1.494(6)$ \\
$\mathrm{C}(1)-\mathrm{N}(1) \# 1$ & $1.316(5)$ \\
$\mathrm{C}(1)-\mathrm{C}(2)$ & $1.524(9)$ \\
$\mathrm{C}(2)-\mathrm{N}(2) \# 1$ & $1.311(5)$ \\
$\mathrm{C}(3)-\mathrm{C}(4)$ & $1.527(8)$ \\
$\mathrm{C}(4)-\mathrm{C}(5)$ & $1.519(7)$ \\
$\mathrm{C}(5)-\mathrm{C}(6)$ & $1.504(7)$ \\
$\mathrm{C}(7)-\mathrm{C}(8)$ & $1.524(7)$ \\
$\mathrm{C}(8)-\mathrm{C}(9)$ & $1.531(7)$ \\
$\mathrm{C}(9)-\mathrm{C}(10)$ & $1.529(7)$ \\
$\mathrm{C}(1)-\mathrm{N}(1)-\mathrm{C}(3)$ & \\
$\mathrm{C}(1)-\mathrm{N}(1)-\mathrm{C}(6)$ & $126.2(5)$ \\
$\mathrm{C}(3)-\mathrm{N}(1)-\mathrm{C}(6)$ & $123.1(4)$
\end{tabular}




$\begin{array}{ll}\mathrm{C}(2)-\mathrm{N}(2)-\mathrm{C}(10) & 125.1(4) \\ \mathrm{C}(2)-\mathrm{N}(2)-\mathrm{C}(7) & 123.1(4) \\ \mathrm{C}(10)-\mathrm{N}(2)-\mathrm{C}(7) & 111.5(4) \\ \mathrm{N}(1)-\mathrm{C}(1)-\mathrm{N}(1) \# 1 & 128.3(7) \\ \mathrm{N}(1)-\mathrm{C}(1)-\mathrm{C}(2) & 115.9(3) \\ \mathrm{N}(1) \# 1-\mathrm{C}(1)-\mathrm{C}(2) & 115.9(3) \\ \mathrm{N}(2) \# 1-\mathrm{C}(2)-\mathrm{N}(2) & 127.9(7) \\ \mathrm{N}(2) \# 1-\mathrm{C}(2)-\mathrm{C}(1) & 116.1(3) \\ \mathrm{N}(2)-\mathrm{C}(2)-\mathrm{C}(1) & 116.1(3) \\ \mathrm{N}(1)-\mathrm{C}(3)-\mathrm{C}(4) & 102.4(4) \\ \mathrm{C}(5)-\mathrm{C}(4)-\mathrm{C}(3) & 103.0(4) \\ \mathrm{C}(6)-\mathrm{C}(5)-\mathrm{C}(4) & 102.5(4) \\ \mathrm{N}(1)-\mathrm{C}(6)-\mathrm{C}(5) & 103.8(4) \\ \mathrm{N}(2)-\mathrm{C}(7)-\mathrm{C}(8) & 102.8(4) \\ \mathrm{C}(7)-\mathrm{C}(8)-\mathrm{C}(9) & 102.9(4) \\ \mathrm{C}(10)-\mathrm{C}(9)-\mathrm{C}(8) & 103.3(4) \\ \mathrm{N}(2)-\mathrm{C}(10)-\mathrm{C}(9) & 102.2(4)\end{array}$

Symmetry transformations used to generate equivalent atoms:

$\# 1-\mathrm{x}+1, \mathrm{y},-\mathrm{z}+3 / 2$

Table S35. Anisotropic displacement parameters $\left(\AA^{2} \times 10^{3}\right)$ for [TPyE $]^{2+2}[1]$. The anisotropic displacement factor exponent takes the form: $-2 \pi^{2}\left[h^{2} a^{* 2} U^{11}+\ldots+2 h k a^{*} b^{*} U^{12}\right]$

\begin{tabular}{lcccccc}
\hline & $\mathrm{U}^{11}$ & $\mathrm{U}^{22}$ & $\mathrm{U}^{33}$ & $\mathrm{U}^{23}$ & $\mathrm{U}^{13}$ & $\mathrm{U}^{12}$ \\
\hline $\mathrm{I}(1)$ & $23(1)$ & $36(1)$ & $20(1)$ & $2(1)$ & $9(1)$ & $5(1)$ \\
$\mathrm{N}(1)$ & $16(2)$ & $7(2)$ & $20(2)$ & $-3(1)$ & $9(2)$ & $-1(1)$ \\
$\mathrm{N}(2)$ & $13(2)$ & $8(2)$ & $16(2)$ & $0(1)$ & $5(2)$ & $0(1)$ \\
$\mathrm{C}(1)$ & $8(3)$ & $14(3)$ & $18(3)$ & 0 & $3(2)$ & 0 \\
$\mathrm{C}(2)$ & $15(3)$ & $13(3)$ & $15(3)$ & 0 & $9(2)$ & 0 \\
$\mathrm{C}(3)$ & $23(2)$ & $4(2)$ & $29(3)$ & $-3(2)$ & $11(2)$ & $-2(2)$ \\
$\mathrm{C}(4)$ & $28(3)$ & $6(2)$ & $27(3)$ & $-5(2)$ & $11(2)$ & $-2(2)$ \\
$\mathrm{C}(5)$ & $27(3)$ & $17(2)$ & $29(3)$ & $-8(2)$ & $17(2)$ & $-2(2)$ \\
$\mathrm{C}(6)$ & $21(2)$ & $13(2)$ & $21(2)$ & $-4(2)$ & $10(2)$ & $-4(2)$ \\
$\mathrm{C}(7)$ & $12(2)$ & $18(2)$ & $22(2)$ & $4(2)$ & $4(2)$ & $4(2)$ \\
$\mathrm{C}(8)$ & $14(2)$ & $20(2)$ & $20(2)$ & $-1(2)$ & $3(2)$ & $-2(2)$ \\
$\mathrm{C}(9)$ & $19(2)$ & $14(2)$ & $18(2)$ & $-3(2)$ & $4(2)$ & $-5(2)$ \\
$\mathrm{C}(10)$ & $17(2)$ & $12(2)$ & $19(2)$ & $-2(2)$ & $6(2)$ & $0(2)$ \\
& & & & & & \\
\hline
\end{tabular}

Table S36. Torsion angles $\left[{ }^{\circ}\right]$ for $[\mathrm{TPyE}]^{2+} 2[\mathrm{I}]$.

\begin{tabular}{lc}
\hline $\mathrm{C}(3)-\mathrm{N}(1)-\mathrm{C}(1)-\mathrm{N}(1) \# 1$ & $13.5(4)$ \\
$\mathrm{C}(6)-\mathrm{N}(1)-\mathrm{C}(1)-\mathrm{N}(1) \# 1$ & $-171.4(5)$ \\
$\mathrm{C}(3)-\mathrm{N}(1)-\mathrm{C}(1)-\mathrm{C}(2)$ & $-166.5(4)$ \\
$\mathrm{C}(6)-\mathrm{N}(1)-\mathrm{C}(1)-\mathrm{C}(2)$ & $8.6(5)$ \\
$\mathrm{C}(10)-\mathrm{N}(2)-\mathrm{C}(2)-\mathrm{N}(2) \# 1$ & $17.6(3)$ \\
$\mathrm{C}(7)-\mathrm{N}(2)-\mathrm{C}(2)-\mathrm{N}(2) \# 1$ & $-169.3(5)$ \\
$\mathrm{C}(10)-\mathrm{N}(2)-\mathrm{C}(2)-\mathrm{C}(1)$ & $-162.4(3)$ \\
$\mathrm{C}(7)-\mathrm{N}(2)-\mathrm{C}(2)-\mathrm{C}(1)$ & $10.7(5)$ \\
$\mathrm{N}(1)-\mathrm{C}(1)-\mathrm{C}(2)-\mathrm{N}(2) \# 1$ & $77.4(3)$ \\
$\mathrm{N}(1) \# 1-\mathrm{C}(1)-\mathrm{C}(2)-\mathrm{N}(2) \# 1$ & $-102.6(3)$ \\
$\mathrm{N}(1)-\mathrm{C}(1)-\mathrm{C}(2)-\mathrm{N}(2)$ & $-102.6(3)$
\end{tabular}


$\mathrm{N}(1) \# 1-\mathrm{C}(1)-\mathrm{C}(2)-\mathrm{N}(2)$

77.4(3)

$\mathrm{C}(1)-\mathrm{N}(1)-\mathrm{C}(3)-\mathrm{C}(4)$

$162.0(4)$

$\mathrm{C}(6)-\mathrm{N}(1)-\mathrm{C}(3)-\mathrm{C}(4)$

$-13.6(5)$

$\mathrm{N}(1)-\mathrm{C}(3)-\mathrm{C}(4)-\mathrm{C}(5)$

$34.3(5)$

$\mathrm{C}(3)-\mathrm{C}(4)-\mathrm{C}(5)-\mathrm{C}(6)$

$-42.7(5)$

$\mathrm{C}(1)-\mathrm{N}(1)-\mathrm{C}(6)-\mathrm{C}(5)$

171.6(4)

$\mathrm{C}(3)-\mathrm{N}(1)-\mathrm{C}(6)-\mathrm{C}(5)$

$-12.6(5)$

$\mathrm{C}(4)-\mathrm{C}(5)-\mathrm{C}(6)-\mathrm{N}(1)$

$33.7(5)$

$\mathrm{C}(2)-\mathrm{N}(2)-\mathrm{C}(7)-\mathrm{C}(8)$

$173.6(4)$

$\mathrm{C}(10)-\mathrm{N}(2)-\mathrm{C}(7)-\mathrm{C}(8)$

$-12.5(5)$

$\mathrm{N}(2)-\mathrm{C}(7)-\mathrm{C}(8)-\mathrm{C}(9)$

$33.2(5)$

$\mathrm{C}(7)-\mathrm{C}(8)-\mathrm{C}(9)-\mathrm{C}(10)$

$-42.3(5)$

$\mathrm{C}(2)-\mathrm{N}(2)-\mathrm{C}(10)-\mathrm{C}(9)$

$160.5(4)$

$-13.3(5)$

$\mathrm{C}(8)-\mathrm{C}(9)-\mathrm{C}(10)-\mathrm{N}(2)$

$33.8(5)$

Symmetry transformations used to generate equivalent atoms:

$\# 1-\mathrm{x}+1, \mathrm{y},-\mathrm{z}+3 / 2$ 


\section{SXXII. Computational Details and Coordinates}

DFT calculations were carried out with the Gaussian16 software package. ${ }^{24}$ The hybrid meta-GGA M06 functional ${ }^{25}$ was selected, given its proven track record to model the structures and energies of main group compounds. ${ }^{25}$ Structures were fully optimized without any geometry or symmetry constraints, using the $6-31+\mathrm{G}^{* *}$ basis set for all elements. ${ }^{26}$ Vibrational frequencies were computed at the same level of theory to classify all stationary points as energy minima, with only real frequencies. These calculations were also used to obtain the thermochemistry corrections (zero-point, thermal, and entropy energies) at $298.15 \mathrm{~K}$. The energies reported were obtained by adding the thermochemistry corrections to the refined potential energies.

To test hypotheses about the structures of reductants, a number of compounds were considered computationally in addition to TPyE, TPiE, TME, TAzE. Specifically, we performed calculations on: (i) the known reductant TDAE, (ii) a variant of TDAE in which two nitrogen atoms are part of the same five-membered heterocyclic ring (TDAE 2.0), and (iii) TAzetE, in which the nitrogen atoms of the tetraaminoethylene scaffold are incorporated into four-membered azetidine rings (Figure S63).

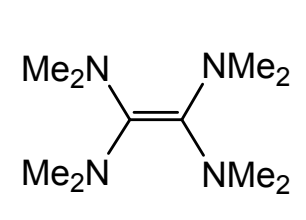

TDAE

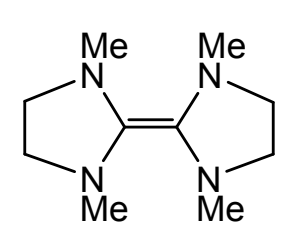

TDAE 2.0

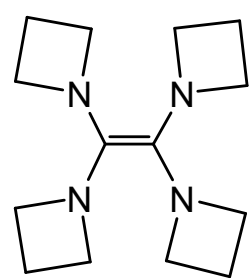

TAzetE

Figure S63: Reductants in addition to TPIE, TPyE, TME, and TAzE used in calculations.

Initially, we explored the ability of the tetraminoethylene scaffolds to undergo the Wanzlick equilibrium and form free carbenes. ${ }^{27}$ The formation of carbenes would represent a problem in catalysis as the reactive carbenes could either undergo deleterious side reactions with substrates or bind to a transition metal catalyst. The formation of a free carbene from TDAE is uphill by 40.8 kcal mol-1 ${ }^{-1}$ consistent with its compatibility with transition metal catalyzed reactions. In contrast, for TDAE 2.0, the formation of a free carbene is uphill by just $14.1 \mathrm{kcal} \mathrm{mol}^{-1}$. This likely explains why attempts to use TDAE 2.0 in catalysis were not successful. In contrast, as shown in Table $\mathrm{S} 37$, it requires at least $36 \mathrm{kcal} \mathrm{mol}^{-1}$ to generate a carbene from TPyE, TPiE, TME, or TAzE, which is consistent with their excellent performance in catalysis. 
Table S37. Calculated gas phase free energies for formation of free carbenes from TPyE, TPiE, TME, or TAzE.
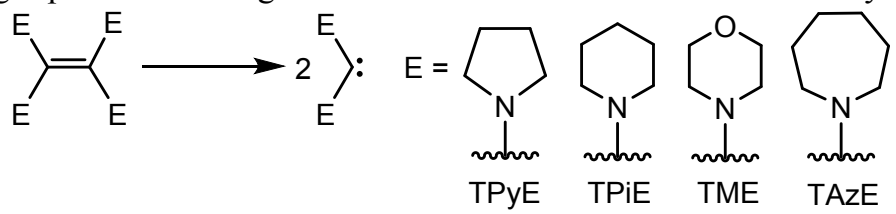

\begin{tabular}{cc}
\hline Reductant & $\boldsymbol{\Delta} \mathbf{G}\left(\mathbf{k c a l ~ \mathbf { ~ m o l } ^ { - 1 } )}\right.$ \\
\hline TPyE & 36.6 \\
TPiE & 45.6 \\
TME & 54.2 \\
TAzE & 38.1 \\
\hline
\end{tabular}

We analyzed the calculated geometries of TDAE, TDAE 2.0, TAzetE, TPyE, TME, and TAzE, as well as their oxidized forms. In general, good agreement is observed between the calculated and experimental structures. For all of the reductants calculated, the molecules contained a central C$\mathrm{C}$ double bond and four $\mathrm{C}-\mathrm{N}$ single bonds from the central $\mathrm{C}-\mathrm{C}$ double bond. The major difference between the structures is related to their planarity. As described in the paper, we quantify planarity by measuring the angle formed by the planes defined by $\mathrm{N}(1)-\mathrm{C}(1)-\mathrm{C}(2)-\mathrm{N}(2)$ and $\mathrm{N}(3)-\mathrm{C}(2)-\mathrm{C}(1)-$ $\mathrm{N}(4)$. As this angle approaches $0^{\circ}$, the molecule become more planar. Figure S64 shows the two planes used for the measurements in TAzetE and TPiE, while the calculated angles between the planes are given for all reductants in Table S38. The calculated data clearly shows that the angle between the planes is the smallest in TAzetE and TDAE 2.0, which are the reductants with the least steric bulk. On this basis, we propose that the reason for the larger deviation from planarity in TDAE, TPyE, TPiE, TME, and TAzE, is related to steric factors.

We also calculated the angle formed by the planes defined by $\mathrm{N}(1)-\mathrm{C}(1)-\mathrm{C}(2)-\mathrm{N}(2)$ and $\mathrm{N}(3)-\mathrm{C}(2)-$ $\mathrm{C}(1)-\mathrm{N}(4)$ for the oxidized forms of the reductants, specifically $\mathrm{TDAE}^{2+}$, TDAE $2.0^{2+}$, TAzetE ${ }^{2+}$, $\mathrm{TPyE}^{2+}, \mathrm{TME}^{2+}$, and $\mathrm{TAzE}^{2+}$. These values are provided in Table S39. By comparing the data in Tables S38 and S39, it can be seen that the strongest reductants, TDAE 2.0, TAzetE, and TPyE, are those with the largest difference in the magnitude of this angle between the neutral and oxidized forms. Given that we do not have experimental data for TDAE 2.0 and TAzetE, in these cases we calculated the reduction potentials using DFT, ${ }^{28}$ whereas for other compounds we used the experimental values. Finally, the HOMOs for all reductants and the LUMOs for the oxidized forms 
of all reductants are shown in Figures S65 and S66. The character of the orbitals is the same across the series, indicating that electrons are being removed from similar types of orbitals on oxidation.

Table S38. Calculated angles between planes formed by $\mathrm{N}(1)-\mathrm{C}(1)-\mathrm{C}(2)-\mathrm{N}(2)$ and $\mathrm{N}(3)-\mathrm{C}(2)-\mathrm{C}(1)-\mathrm{N}(4)$ for different reductants.

\begin{tabular}{cc}
\hline Reductant & Angle $\left(^{\circ}\right)$ \\
\hline TAzetE & 11.3 \\
TPyE & 23.7 \\
TPiE & 20.1 \\
TME & 24.7 \\
TAzE & 24.6 \\
TDAE & 27.8 \\
TDAE 2.0 & 4.85 \\
\hline
\end{tabular}

Figure S64. Angles between planes formed by N(1)-C(1)-C(2)-N(2) and N(3)-C(2)-C(1)-N(4) for a) TAzetE and b) TPiE.

a)

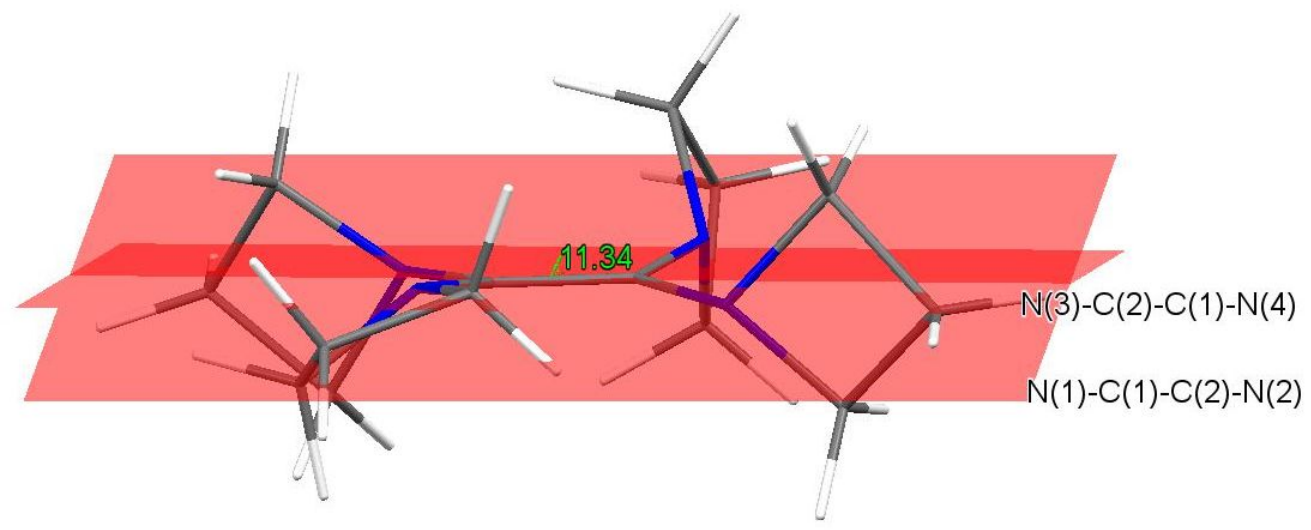

b)

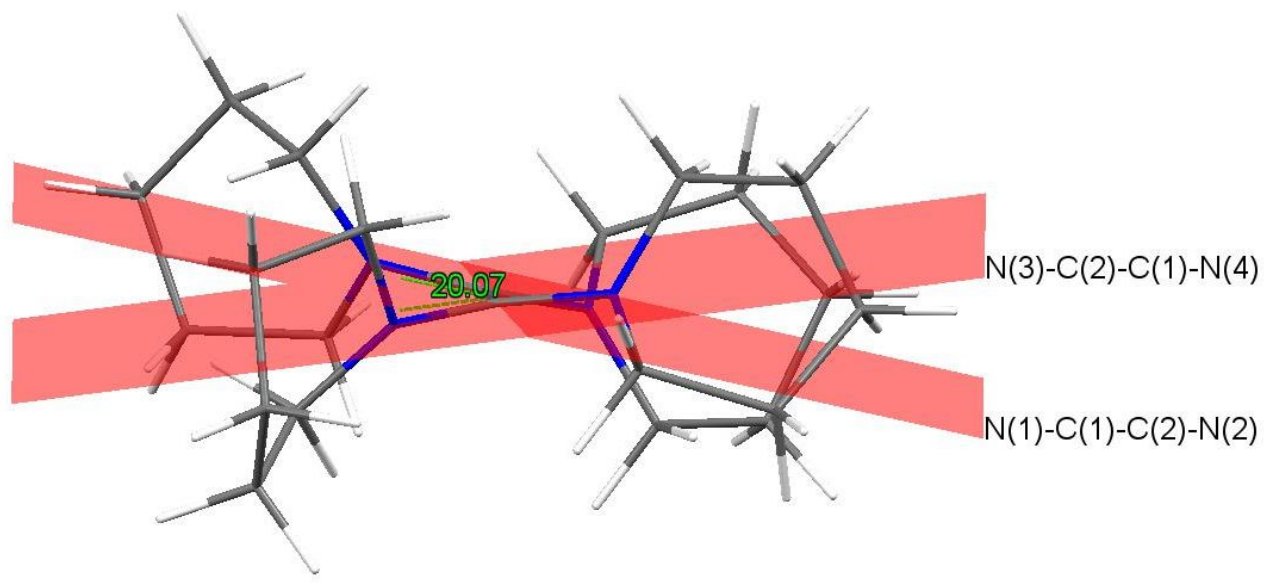


Table S39. Calculated angles between planes formed by N(1)-C(1)-C(2)-N(2) and N(3)-C(2)-C(1)-N(4) for oxidized versions of reductants.

\begin{tabular}{cc}
\hline Reductant & Angle $\left(^{\circ}\right)$ \\
\hline TAzetE $^{2+}$ & 89.9 \\
TPyE $^{2+}$ & 79.5 \\
TPiE $^{2+}$ & 72.5 \\
TME $^{2+}$ & 69.3 \\
TAzE $^{2+}$ & 78.7 \\
TDAE $^{2+}$ & 69.1 \\
TDAE 2.0 & 86.2 \\
\hline
\end{tabular}


a)

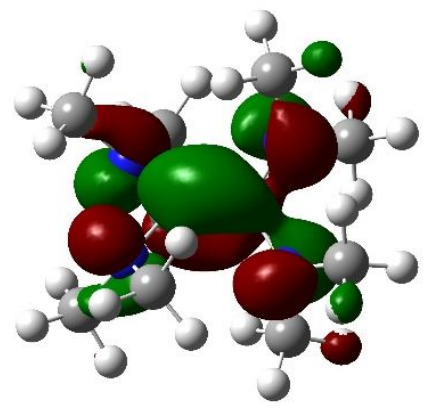

d)

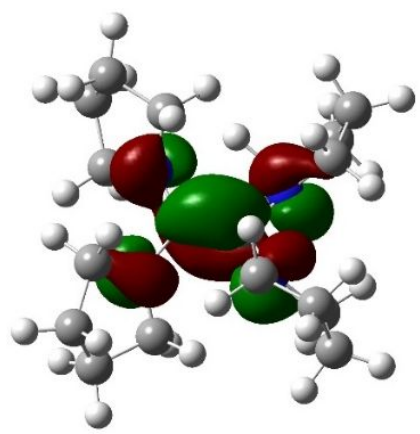

f)

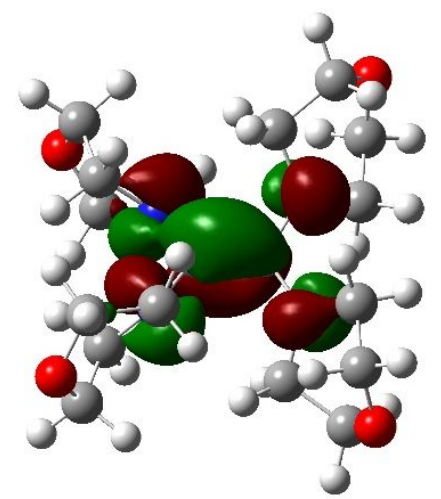

c)
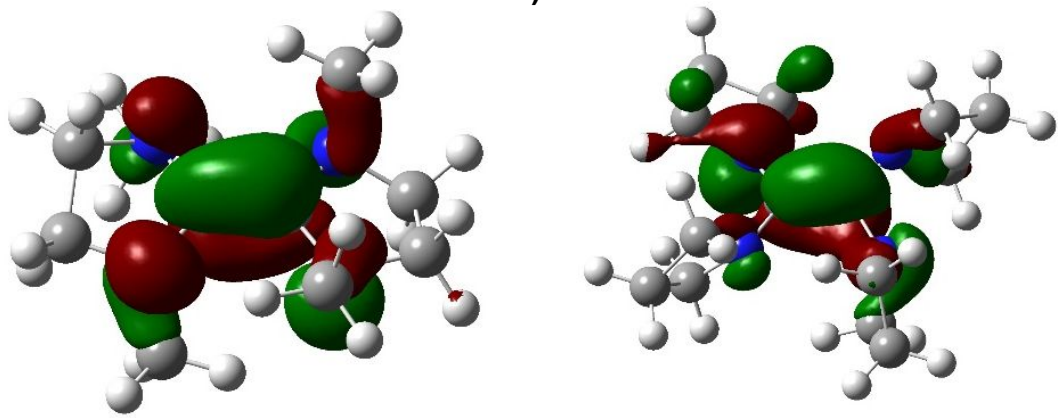

e)

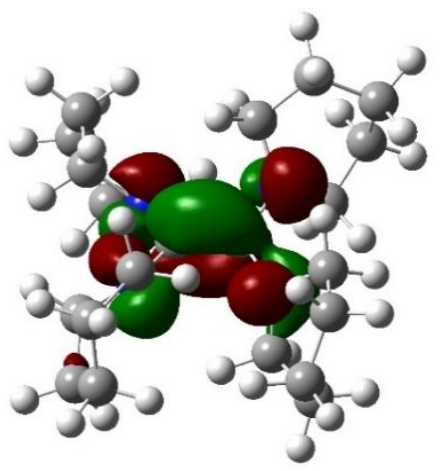

g)

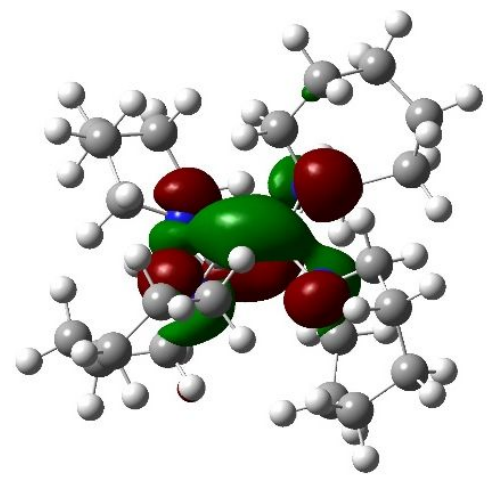

Figure S65: DFT calculated HOMOs of a) TDAE, b) TDAE 2.0, c) TAzetE, d) TPyE, e) TPiE, f) TME, and g) TAzE. 
a)

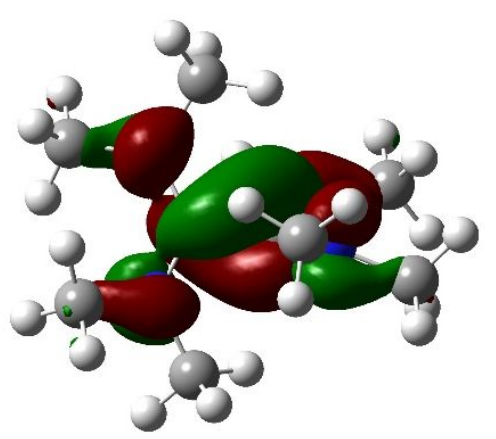

d)

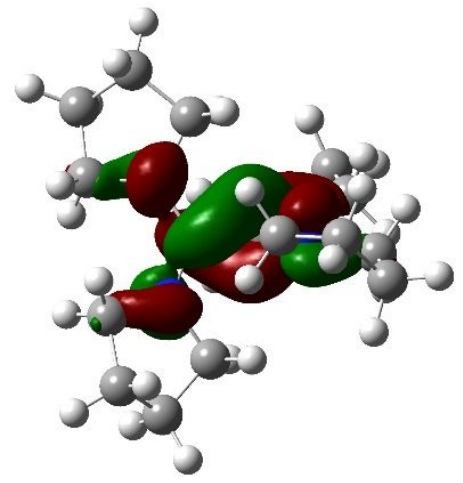

f)

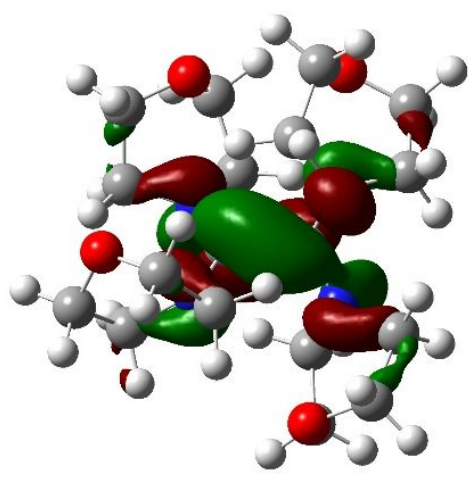

b)

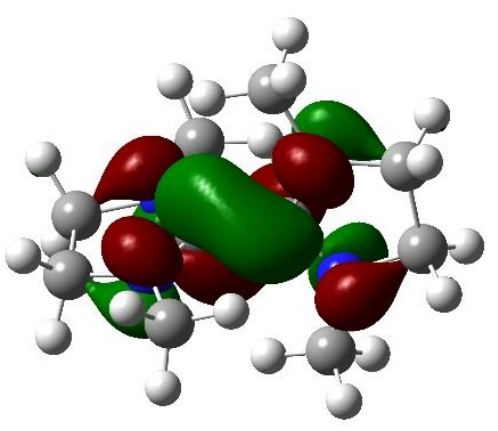

c)

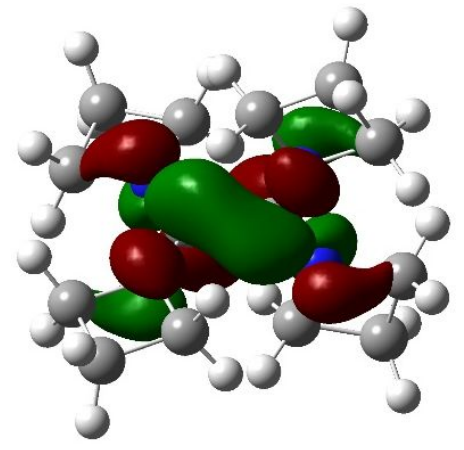

e)

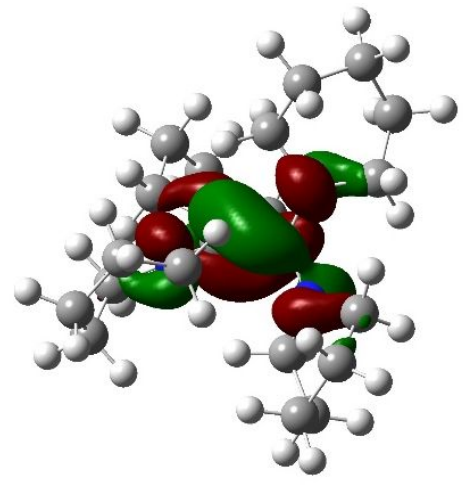

g)

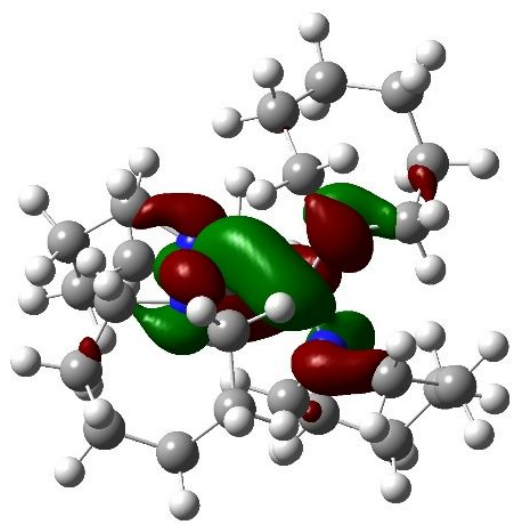

Figure S66: DFT calculated LUMOs of a) $\mathrm{TDAE}^{2+}$, b) TDAE $2.0^{2+}$, c) $\mathrm{TAzetE}^{2+}$, d) $\mathrm{TPyE}^{2+}$, e) $\mathrm{TPiE}^{2+}$, f) $\mathrm{TME}^{2+}$, and g) $\mathrm{TAzE}^{2+}$. 


\section{Optimized Coordinates and DFT Energies}

\begin{tabular}{|c|c|c|c|}
\hline & $G$ & & \\
\hline $\mathrm{C}$ & -0.126000 & -0.706000 & 0.070000 \\
\hline C & -0.009000 & 0.649000 & -0.044000 \\
\hline $\mathrm{C}$ & 1.941000 & 0.917000 & -1.517000 \\
\hline$C$ & 1.553000 & 2.536000 & 0.312000 \\
\hline & 3.222000 & 1.734000 & -1.65200 \\
\hline $\mathrm{H}$ & 1.286000 & 1.068000 & -2.399000 \\
\hline$H$ & 2.195000 & -0.157000 & -1.491000 \\
\hline & 3.035000 & 2.609000 & 0.697000 \\
\hline $\mathrm{H}$ & 1.306000 & 3.379000 & -0.367000 \\
\hline $\mathrm{H}$ & 0.913000 & 2.664000 & 1.192000 \\
\hline$C$ & 3.914000 & 1.838000 & -0.300000 \\
\hline $\mathrm{H}$ & 2.998000 & 2.743000 & -2.030000 \\
\hline & 3.873000 & 1.263000 & -2.399000 \\
\hline & 3.177000 & 2.204000 & 1.708000 \\
\hline & 3.337000 & 3.665000 & 0.738000 \\
\hline & 4.096000 & 0.819000 & 0.075000 \\
\hline $\mathrm{H}$ & 4.899000 & 2.313000 & -0.396000 \\
\hline $\mathrm{C}$ & -1.459000 & 2.328000 & -1.082000 \\
\hline $\mathrm{C}$ & -2.115000 & 5000 & 1.0 \\
\hline $\mathrm{C}$ & -2.629000 & 3.25 & -0.7 \\
\hline $\mathrm{H}$ & -1.699000 & 1.691000 & -1.956000 \\
\hline $\mathrm{H}$ & -0.587000 & 2.937000 & -1.375000 \\
\hline$C$ & -2.524000 & 2.685000 & 1.693000 \\
\hline $\mathrm{H}$ & -3.006000 & 0.812000 & 0.691000 \\
\hline $\mathrm{H}$ & -1.696000 & 0.676000 & 1.861000 \\
\hline $\mathrm{C}$ & -2.452000 & 3.812000 & 0.651000 \\
\hline $\mathrm{H}$ & -3.579000 & 2.697000 & -0.8 \\
\hline $\mathrm{H}$ & -2.686000 & 4.049000 & -1.5 \\
\hline $\mathrm{H}$ & -1.868000 & 2.926000 & 2.540000 \\
\hline $\mathrm{H}$ & -3.543000 & 2.602000 & 2.098000 \\
\hline $\mathrm{H}$ & -1.470000 & 4.307000 & 0.702000 \\
\hline $\mathrm{H}$ & -3.201000 & 4.584000 & 0.865000 \\
\hline $\mathrm{C}$ & -2.171000 & -1.130000 & -1.281000 \\
\hline $\mathrm{C}$ & -1.809000 & -2.328000 & 0.876000 \\
\hline $\mathrm{C}$ & -2.926000 & -2.4 & -1.6 \\
\hline $\mathrm{H}$ & -1.504000 & -0.826000 & -2.1 \\
\hline $\mathrm{H}$ & -2.894000 & -0.299000 & -1.144000 \\
\hline f & -3.328000 & -2.401000 & 0.875000 \\
\hline $\mathrm{H}$ & -1.398000 & -3.340000 & 0.687000 \\
\hline $\mathrm{H}$ & -1.438000 & -2.022000 & 1.867000 \\
\hline $\mathrm{C}$ & -3.866000 & -2.828000 & -0.498000 \\
\hline $\mathrm{H}$ & -2.187000 & -3.195000 & -1.828000 \\
\hline $\mathrm{H}$ & -3.485000 & -2.262 & -2.56900 \\
\hline 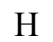 & -3.720000 & -1.409000 & 1.14 \\
\hline H & -3.668000 & -3.092000 & 1.656000 \\
\hline $\mathrm{H}$ & -4.862000 & -2.389000 & -0.651000 \\
\hline $\mathrm{H}$ & -4.004000 & -3.917000 & -0.529000 \\
\hline $\mathrm{C}$ & 1.772000 & -1.259000 & 1.549000 \\
\hline $\mathrm{C}$ & 1.120000 & -2.827000 & -0.248000 \\
\hline 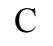 & 2.950000 & -2.226000 & 1.637000 \\
\hline $\mathrm{H}$ & 1.144000 & -1.324000 & 2.459000 \\
\hline & 2.154000 & -0.225000 & 1.497000 \\
\hline
\end{tabular}




$\begin{array}{rrrr}\mathrm{C} & 2.563000 & -3.079000 & -0.697000 \\ \mathrm{H} & 0.807000 & -3.628000 & 0.452000 \\ \mathrm{H} & 0.431000 & -2.882000 & -1.100000 \\ \mathrm{C} & 3.567000 & -2.416000 & 0.258000 \\ \mathrm{H} & 2.623000 & -3.199000 & 2.033000 \\ \mathrm{H} & 3.684000 & -1.833000 & 2.352000 \\ \mathrm{H} & 2.710000 & -2.696000 & -1.716000 \\ \mathrm{H} & 2.735000 & -4.164000 & -0.743000 \\ \mathrm{H} & 3.853000 & -1.427000 & -0.132000 \\ \mathrm{H} & 4.492000 & -3.004000 & 0.318000 \\ \mathrm{~N} & 1.241000 & 1.254000 & -0.285000 \\ \mathrm{~N} & 0.986000 & -1.518000 & 0.353000 \\ \mathrm{~N} & -1.353000 & -1.357000 & -0.106000 \\ \mathrm{~N} & -1.097000 & 1.527000 & 0.081000\end{array}$

$\mathrm{TPiE}^{2+} \mathrm{G}=-680151.4524 \mathrm{kcal} / \mathrm{mol}$

$\begin{array}{llll}\text { C } & -0.082000 & -0.796000 & 0.195000\end{array}$

$\begin{array}{llll}\text { C } & -0.010000 & 0.704000 & -0.024000\end{array}$

$\begin{array}{llll}\text { C } & 1.846000 & 0.227000 & -1.560000\end{array}$

$\begin{array}{llll}\text { C } & 1.534000 & 2.549000 & -0.619000\end{array}$

$\begin{array}{llll}\text { C } & 2.453000 & 0.996000 & -2.719000\end{array}$

$\mathrm{H} \quad 1.216000 \quad-0.594000 \quad-1.921000$

$\mathrm{H} \quad 2.632000 \quad-0.204000 \quad-0.922000$

$\begin{array}{llll}\text { C } & 3.039000 & 2.541000 & -0.810000\end{array}$

$\mathrm{H} \quad 1.059000 \quad 3.199000 \quad-1.363000$

$\begin{array}{llll}\mathrm{H} & 1.266000 & 2.905000 & 0.381000\end{array}$

$\begin{array}{llll}\text { C } & 3.423000 & 2.073000 & -2.216000\end{array}$

$\mathrm{H} \quad 1.638000 \quad 1.439000 \quad-3.308000$

$\mathrm{H} \quad 2.956000 \quad 0.286000 \quad-3.384000$

$\mathrm{H} \quad 3.499000 \quad 1.905000-0.040000$

$\mathrm{H} \quad 3.408000 \quad 3.554000 \quad-0.618000$

$\mathrm{H} \quad 4.449000 \quad 1.689000 \quad-2.204000$

$\mathrm{H} \quad 3.422000 \quad 2.919000 \quad-2.912000$

$\begin{array}{llll}\text { C } & -1.370000 & 2.775000 & -0.024000\end{array}$

$\begin{array}{llll}\text { C } & -1.882000 & 0.871000 & 1.552000\end{array}$

$\begin{array}{llll}\text { C } & -2.857000 & 2.991000 & 0.178000\end{array}$

$\mathrm{H} \quad-1.107000 \quad 2.787000 \quad-1.088000$

$\begin{array}{llll}\mathrm{H} & -0.803000 & 3.564000 & 0.487000\end{array}$

$\begin{array}{llll}\text { C } & -2.367000 & 1.996000 & 2.449000\end{array}$

$\mathrm{H} \quad-2.730000 \quad 0.370000 \quad 1.063000$

$\begin{array}{llll}\mathrm{H} & -1.333000 & 0.120000 & 2.129000\end{array}$

C $\quad-3.228000 \quad 2.995000 \quad 1.663000$

$\mathrm{H} \quad-3.415000 \quad 2.219000 \quad-0.369000$

$\mathrm{H} \quad-3.125000 \quad 3.941000 \quad-0.296000$

$\mathrm{H} \quad-1.491000 \quad 2.489000 \quad 2.891000$

$\mathrm{H} \quad-2.925000 \quad 1.562000 \quad 3.285000$

$\mathrm{H} \quad-3.099000 \quad 3.999000 \quad 2.083000$

$\begin{array}{llll}\mathrm{H} & -4.291000 & 2.754000 & 1.772000\end{array}$

$\begin{array}{llll}\text { C } & -1.713000 & -0.796000 & -1.638000\end{array}$

$\begin{array}{llll}\text { C } & -1.598000 & -2.746000 & -0.039000\end{array}$

$\begin{array}{llll}\text { C } & -2.261000 & -1.869000 & -2.562000\end{array}$

$\mathrm{H} \quad-0.997000 \quad-0.157000 \quad-2.166000$

$\mathrm{H} \quad-2.528000 \quad-0.159000 \quad-1.262000$

$\begin{array}{llll}\text { C } & -3.083000 & -2.774000 & -0.351000\end{array}$

$\mathrm{H} \quad-1.094000 \quad-3.573000 \quad-0.557000$

$\mathrm{H} \quad-1.409000 \quad-2.840000 \quad 1.035000$ 


$\begin{array}{rrrr}\mathrm{C} & -3.338000 & -2.705000 & -1.860000 \\ \mathrm{H} & -1.427000 & -2.499000 & -2.902000 \\ \mathrm{H} & -2.657000 & -1.384000 & -3.460000 \\ \mathrm{H} & -3.578000 & -1.946000 & 0.176000 \\ \mathrm{H} & -3.501000 & -3.690000 & 0.078000 \\ \mathrm{H} & -4.331000 & -2.280000 & -2.043000 \\ \mathrm{H} & -3.354000 & -3.712000 & -2.289000 \\ \mathrm{C} & 1.446000 & -0.476000 & 2.088000 \\ \mathrm{C} & 1.251000 & -2.737000 & 1.003000 \\ \mathrm{C} & 2.661000 & -1.165000 & 2.685000 \\ \mathrm{H} & 0.701000 & -0.251000 & 2.863000 \\ \mathrm{H} & 1.752000 & 0.473000 & 1.627000 \\ \mathrm{C} & 2.762000 & -2.794000 & 0.776000 \\ \mathrm{H} & 0.962000 & -3.218000 & 1.945000 \\ \mathrm{H} & 0.739000 & -3.254000 & 0.194000 \\ \mathrm{C} & 3.533000 & -1.733000 & 1.577000 \\ \mathrm{H} & 2.355000 & -1.952000 & 3.385000 \\ \mathrm{H} & 3.203000 & -0.425000 & 3.284000 \\ \mathrm{H} & 2.971000 & -2.700000 & -0.296000 \\ \mathrm{H} & 3.081000 & -3.804000 & 1.057000 \\ \mathrm{H} & 3.850000 & -0.910000 & 0.918000 \\ \mathrm{H} & 4.454000 & -2.154000 & 1.989000 \\ \mathrm{~N} & 1.010000 & 1.154000 & -0.742000 \\ \mathrm{~N} & 0.797000 & -1.323000 & 1.040000 \\ \mathrm{~N} & -1.025000 & -1.441000 & -0.479000 \\ \mathrm{~N} & -0.979000 & 1.435000 & 0.508000\end{array}$

TPiE Carbene $G=-340207.8826 \mathrm{kcal} / \mathrm{mol}$

$\begin{array}{lrrr}\mathrm{C} & 0.000000 & -1.039000 & -0.001000 \\ \mathrm{C} & -2.197000 & -1.206000 & 0.927000 \\ \mathrm{C} & -1.418000 & 1.033000 & 0.632000 \\ \mathrm{C} & -3.475000 & -0.678000 & 0.246000 \\ \mathrm{H} & -2.316000 & -1.206000 & 2.023000 \\ \mathrm{H} & -1.962000 & -2.226000 & 0.610000 \\ \mathrm{C} & -2.298000 & 1.381000 & -0.572000 \\ \mathrm{H} & -1.991000 & 1.153000 & 1.563000 \\ \mathrm{H} & -0.549000 & 1.684000 & 0.739000 \\ \mathrm{C} & -3.112000 & 0.148000 & -0.985000 \\ \mathrm{H} & -4.054000 & -0.051000 & 0.941000 \\ \mathrm{H} & -4.126000 & -1.518000 & -0.022000 \\ \mathrm{H} & -1.681000 & 1.722000 & -1.416000 \\ \mathrm{H} & -2.963000 & 2.216000 & -0.308000 \\ \mathrm{H} & -2.513000 & -0.475000 & -1.664000 \\ \mathrm{H} & -4.010000 & 0.445000 & -1.539000 \\ \mathrm{C} & 2.197000 & -1.205000 & -0.929000 \\ \mathrm{C} & 1.418000 & 1.034000 & -0.632000 \\ \mathrm{C} & 3.475000 & -0.678000 & -0.244000 \\ \mathrm{H} & 2.318000 & -1.202000 & -2.024000 \\ \mathrm{H} & 1.962000 & -2.225000 & -0.615000 \\ \mathrm{C} & 2.298000 & 1.382000 & 0.572000 \\ \mathrm{H} & 1.991000 & 1.155000 & -1.563000 \\ \mathrm{H} & 0.549000 & 1.684000 & -0.738000 \\ \mathrm{C} & 3.111000 & 0.147000 & 0.986000 \\ \mathrm{H} & 4.056000 & -0.052000 & -0.938000 \\ \mathrm{H} & 4.125000 & -1.520000 & 0.025000 \\ \mathrm{H} & 1.682000 & 1.724000 & 1.416000\end{array}$




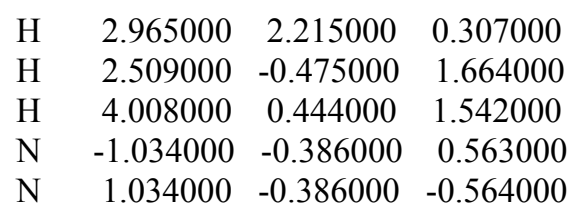

\begin{tabular}{lrrr} 
TPyE & \multicolumn{3}{c}{$\mathrm{G}=-581540.4649 \mathrm{kcal} / \mathrm{mol}$} \\
$\mathrm{C}$ & -0.073000 & -0.678000 & -0.151000 \\
$\mathrm{C}$ & 0.074000 & 0.678000 & -0.151000 \\
$\mathrm{C}$ & 1.304000 & -2.609000 & 0.591000 \\
$\mathrm{C}$ & 2.087000 & -1.297000 & -1.274000 \\
$\mathrm{C}$ & 2.726000 & -3.033000 & 0.213000 \\
$\mathrm{H}$ & 0.596000 & -3.453000 & 0.516000 \\
$\mathrm{H}$ & 1.241000 & -2.233000 & 1.627000 \\
$\mathrm{C}$ & 2.848000 & -2.615000 & -1.254000 \\
$\mathrm{H}$ & 2.736000 & -0.467000 & -0.928000 \\
$\mathrm{H}$ & 1.707000 & -1.014000 & -2.266000 \\
$\mathrm{H}$ & 3.455000 & -2.471000 & 0.813000 \\
$\mathrm{H}$ & 2.912000 & -4.099000 & 0.381000 \\
$\mathrm{H}$ & 3.886000 & -2.519000 & -1.589000 \\
$\mathrm{H}$ & 2.345000 & -3.347000 & -1.901000 \\
$\mathrm{C}$ & 1.850000 & 2.357000 & -0.698000 \\
$\mathrm{C}$ & 2.073000 & 1.032000 & 1.313000 \\
$\mathrm{C}$ & 3.186000 & 2.654000 & -0.023000 \\
$\mathrm{H}$ & 1.193000 & 3.247000 & -0.695000 \\
$\mathrm{H}$ & 1.965000 & 2.048000 & -1.751000 \\
$\mathrm{C}$ & 2.920000 & 2.293000 & 1.438000 \\
$\mathrm{H}$ & 2.717000 & 0.133000 & 1.230000 \\
$\mathrm{H}$ & 1.399000 & 0.866000 & 2.169000 \\
$\mathrm{H}$ & 3.966000 & 1.995000 & -0.430000 \\
$\mathrm{H}$ & 3.516000 & 3.689000 & -0.166000 \\
$\mathrm{H}$ & 3.833000 & 2.148000 & 2.026000 \\
$\mathrm{H}$ & 2.329000 & 3.088000 & 1.916000 \\
$\mathrm{C}$ & -2.087000 & 1.296000 & -1.275000 \\
$\mathrm{C}$ & -1.304000 & 2.609000 & 0.591000 \\
$\mathrm{C}$ & -2.848000 & 2.615000 & -1.254000 \\
$\mathrm{H}$ & -2.735000 & 0.467000 & -0.929000 \\
$\mathrm{H}$ & -1.706000 & 1.014000 & -2.267000 \\
$\mathrm{C}$ & -2.726000 & 3.031000 & 0.212000 \\
$\mathrm{H}$ & -0.596000 & 3.453000 & 0.514000 \\
$\mathrm{H}$ & -1.240000 & 2.233000 & 1.627000 \\
$\mathrm{H}$ & -2.345000 & 3.347000 & -1.901000 \\
$\mathrm{H}$ & -3.886000 & 2.518000 & -1.589000 \\
$\mathrm{H}$ & -2.913000 & 4.097000 & 0.381000 \\
$\mathrm{H}$ & -3.455000 & 2.469000 & 0.813000 \\
$\mathrm{C}$ & -1.849000 & -2.357000 & -0.698000 \\
$\mathrm{C}$ & -2.073000 & -1.031000 & 1.313000 \\
$\mathrm{C}$ & -3.187000 & -2.653000 & -0.023000 \\
$\mathrm{H}$ & -1.194000 & -3.247000 & -0.693000 \\
$\mathrm{H}$ & -1.964000 & -2.048000 & -1.750000 \\
$\mathrm{C}$ & -2.922000 & -2.291000 & 1.438000 \\
$\mathrm{H}$ & -2.717000 & -0.131000 & 1.229000 \\
$\mathrm{H}$ & -1.400000 & -0.865000 & 2.169000 \\
$\mathrm{H}$ & -3.966000 & -1.993000 & -0.431000 \\
$\mathrm{H}$ & -3.517000 & -3.687000 & -0.166000 \\
$\mathrm{H}$ & -3.835000 & -2.145000 & 2.025000
\end{tabular}




\begin{tabular}{|c|c|c|c|}
\hline $\mathrm{H}$ & -2.331000 & -3.086000 & 1.917000 \\
\hline $\mathrm{N}$ & -0.983000 & 1.564000 & -0.376000 \\
\hline $\mathrm{N}$ & 1.307000 & 1.278000 & 0.108000 \\
\hline $\mathrm{N}$ & 0.984000 & -1.564000 & -0.376000 \\
\hline V & -1.306000 & -1.278000 & 0.108000 \\
\hline \multicolumn{4}{|c|}{$T P y E^{2+} G=-581232.1278 \mathrm{kcal} / \mathrm{mol}$} \\
\hline $\mathrm{C}$ & -0.000000 & 0.756000 & -0.000000 \\
\hline $\mathrm{C}$ & 0.000000 & -0.756000 & -0.000000 \\
\hline $\mathrm{C}$ & -1.399000 & 2.749000 & -0.590000 \\
\hline $\mathrm{C}$ & -1.765000 & 0.606000 & -1.729000 \\
\hline $\mathrm{C}$ & -2.781000 & 2.696000 & -1.224000 \\
\hline $\mathrm{H}$ & -0.743000 & 3.446000 & -1.123000 \\
\hline $\mathrm{H}$ & -1.410000 & 3.000000 & 0.475000 \\
\hline $\mathrm{C}$ & -2.597000 & 1.707000 & -2.367000 \\
\hline $\mathrm{H}$ & -2.401000 & -0.108000 & -1.185000 \\
\hline $\mathrm{H}$ & -1.142000 & 0.053000 & -2.440000 \\
\hline $\mathrm{H}$ & -3.519000 & 2.323000 & -0.503000 \\
\hline $\mathrm{H}$ & -3.110000 & 3.685000 & -1.554000 \\
\hline $\mathrm{H}$ & -3.538000 & 1.323000 & -2.767000 \\
\hline $\mathrm{H}$ & -2.046000 & 2.169000 & -3.196000 \\
\hline $\mathrm{C}$ & -1.397000 & -2.750000 & 0.590000 \\
\hline $\mathrm{C}$ & -1.764000 & -0.607000 & 1.729000 \\
\hline $\mathrm{C}$ & -2.779000 & -2.698000 & 1.225000 \\
\hline $\mathrm{H}$ & -0.741000 & -3.447000 & 1.123000 \\
\hline $\mathrm{H}$ & -1.408000 & -3.001000 & -0.476000 \\
\hline $\mathrm{C}$ & -2.595000 & -1.709000 & 2.367000 \\
\hline $\mathrm{H}$ & -2.401000 & 0.106000 & 1.185000 \\
\hline $\mathrm{H}$ & -1.142000 & -0.054000 & 2.441000 \\
\hline $\mathrm{H}$ & -3.517000 & -2.325000 & 0.503000 \\
\hline $\mathrm{H}$ & -3.108000 & -3.687000 & 1.554000 \\
\hline $\mathrm{H}$ & -3.537000 & -1.326000 & 2.768000 \\
\hline $\mathrm{H}$ & -2.044000 & -2.171000 & 3.196000 \\
\hline $\mathrm{C}$ & 1.765000 & -0.606000 & -1.729000 \\
\hline $\mathrm{C}$ & 1.399000 & -2.749000 & -0.590000 \\
\hline $\mathrm{C}$ & 2.597000 & -1.707000 & -2.367000 \\
\hline $\mathrm{H}$ & 2.401000 & 0.108000 & -1.185000 \\
\hline $\mathrm{H}$ & 1.142000 & -0.053000 & -2.441000 \\
\hline $\mathrm{C}$ & 2.781000 & -2.696000 & -1.224000 \\
\hline $\mathrm{H}$ & 0.743000 & -3.446000 & -1.123000 \\
\hline $\mathrm{H}$ & 1.410000 & -3.000000 & 0.476000 \\
\hline $\mathrm{H}$ & 2.046000 & -2.170000 & -3.195000 \\
\hline $\mathrm{H}$ & 3.538000 & -1.323000 & -2.767000 \\
\hline $\mathrm{H}$ & 3.111000 & -3.685000 & -1.554000 \\
\hline $\mathrm{H}$ & 3.519000 & -2.323000 & -0.503000 \\
\hline $\mathrm{C}$ & 1.397000 & 2.750000 & 0.590000 \\
\hline $\mathrm{C}$ & 1.764000 & 0.607000 & 1.729000 \\
\hline $\mathrm{C}$ & 2.778000 & 2.699000 & 1.224000 \\
\hline $\mathrm{H}$ & 0.740000 & 3.447000 & 1.122000 \\
\hline $\mathrm{H}$ & 1.408000 & 3.001000 & -0.476000 \\
\hline $\mathrm{C}$ & 2.595000 & 1.709000 & 2.367000 \\
\hline $\mathrm{H}$ & 2.401000 & -0.106000 & 1.185000 \\
\hline $\mathrm{H}$ & 1.142000 & 0.054000 & 2.440000 \\
\hline $\mathrm{H}$ & 3.517000 & 2.325000 & 0.503000 \\
\hline $\mathrm{H}$ & 3.10 & 3.68 & 1. \\
\hline
\end{tabular}




$\begin{array}{rrrr}\mathrm{H} & 3.537000 & 1.326000 & 2.768000 \\ \mathrm{H} & 2.044000 & 2.171000 & 3.195000 \\ \mathrm{~N} & 0.909000 & -1.348000 & -0.755000 \\ \mathrm{~N} & -0.908000 & -1.349000 & 0.755000 \\ \mathrm{~N} & -0.909000 & 1.348000 & -0.755000 \\ \mathrm{~N} & 0.908000 & 1.349000 & 0.755000\end{array}$

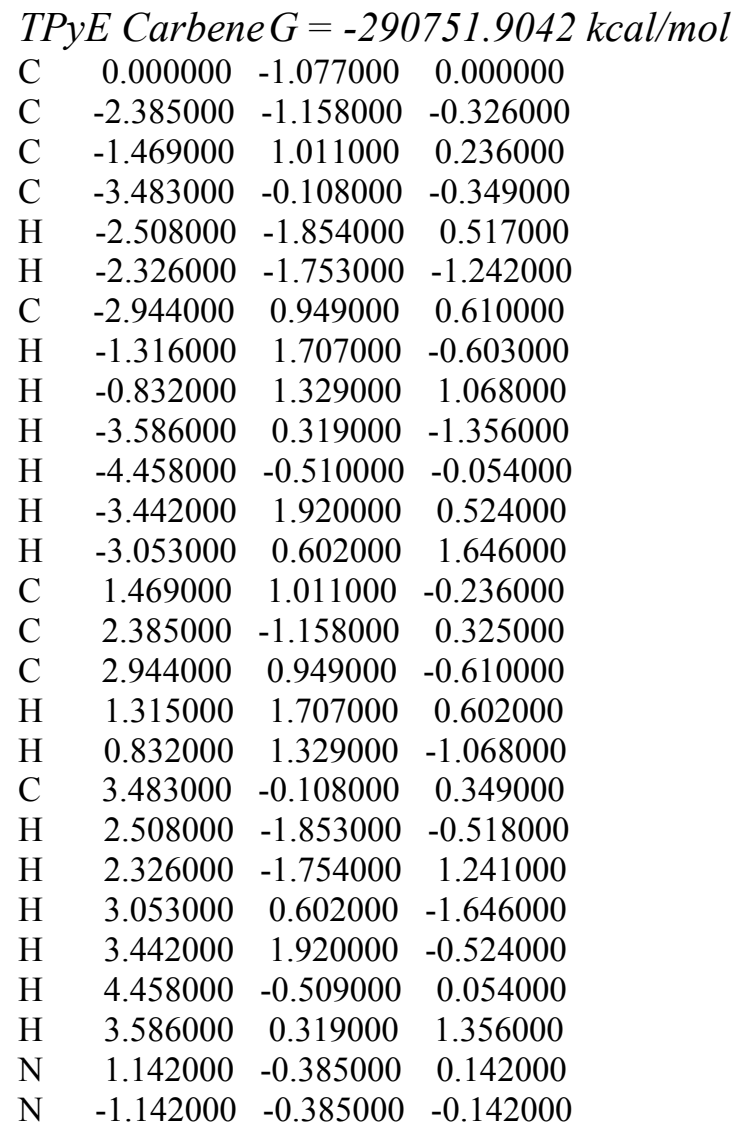

TME $\quad G=-770976.3827 \mathrm{kcal} / \mathrm{mol}$

$\begin{array}{llll}\text { C } & -0.000000 & -0.801000 & 0.000000\end{array}$

$\begin{array}{llll}\text { C } & 0.000000 & 0.564000 & 0.000000\end{array}$

$\begin{array}{llll}\text { C } & 1.971000 & 1.169000 & -1.404000\end{array}$

$\begin{array}{llll}\text { C } & 1.524000 & 2.382000 & 0.700000\end{array}$

$\begin{array}{llll}\text { C } & 2.418000 & 2.542000 & -1.864000\end{array}$

$\mathrm{H} \quad 1.350000 \quad 0.701000 \quad-2.182000$

$\mathrm{H} \quad 2.856000 \quad 0.516000 \quad-1.266000$

C $\quad 2.960000 \quad 2.798000 \quad 0.452000$

$\mathrm{H} \quad 0.864000 \quad 3.262000 \quad 0.575000$

$\mathrm{H} \quad 1.417000 \quad 2.050000 \quad 1.746000$

$\mathrm{H} \quad 1.526000 \quad 3.144000 \quad-2.114000$

$\mathrm{H} \quad 3.041000 \quad 2.471000 \quad-2.764000$

$\begin{array}{llll}\mathrm{H} & 3.634000 & 1.959000 & 0.699000\end{array}$

$\mathrm{H} \quad 3.231000 \quad 3.641000 \quad 1.098000$

$\begin{array}{llll}\text { C } & -1.524000 & 2.382000 & -0.700000\end{array}$

$\begin{array}{llll}\text { C } & -1.971000 & 1.169000 & 1.404000\end{array}$

$\begin{array}{llll}\mathrm{C} & -2.960000 & 2.799000 & -0.453000\end{array}$

$\mathrm{H} \quad-1.417000 \quad 2.049000 \quad-1.747000$ 


\begin{tabular}{|c|c|c|c|}
\hline H & -0.864000 & 3.262000 & -0.576000 \\
\hline & -2.417000 & 2.543000 & 363000 \\
\hline & -2.856000 & 0.517000 & 1.266000 \\
\hline & -1.350000 & 0.702000 & 2.183000 \\
\hline & -3.634000 & 1.959000 & -0.698000 \\
\hline & -3.231000 & 3.641000 & -1.09800 \\
\hline & -1.526000 & 3.144000 & 2.114000 \\
\hline & -3.041000 & 2.472000 & 2.763000 \\
\hline & -2.098000 & -1.124000 & -1.283000 \\
\hline & -1.537000 & -2.657000 & 0.567000 \\
\hline & -2.896000 & -2.325000 & 5000 \\
\hline & & & \\
\hline & -2.778000 & 000 & -0.8 \\
\hline & -3.0 & -2.7 & \\
\hline & -1.1 & -3.626000 & 0.2 \\
\hline & -1.1 & -2.479000 & 1.2 \\
\hline & -2.2 & -3.059000 & -2.205000 \\
\hline & -3.6 & -2.0 & -2.500000 \\
\hline & -3.4 & & 1.054000 \\
\hline & -3.3 & & \\
\hline & 2.0 & -1 . & \\
\hline & 1.5 & -2.6 & -0 . \\
\hline & 2.89 & -2.3 & 1.7 \\
\hline & 1.5 & -0.6 & \\
\hline & & & \\
\hline & 3.0 & -2.7 & -0. \\
\hline & & & \\
\hline & & & \\
\hline & & -3 & \\
\hline & 3.6 & $-2 .($ & \\
\hline & 3.45 & -1.8 & -1. \\
\hline & 3.36 & -3.5 & -1 . \\
\hline & & & \\
\hline & 000 & -1.5 & 00 \\
\hline & & -1.5 & -0 \\
\hline & & & \\
\hline & -2 & -2 & -0 . \\
\hline & & & -0.8 \\
\hline & 3.60 & -2.9 & 0.6 \\
\hline & -3.1 & 3.223000 & 0.880000 \\
\hline \multicolumn{4}{|c|}{$T M E^{2+} G=-770638.714 \mathrm{kcal} / \mathrm{mol}$} \\
\hline C & -0.26 & -0.94 & -0.009000 \\
\hline c & -0.15 & & 000 \\
\hline C & & & \\
\hline & & & \\
\hline & & & \\
\hline & 1.409000 & 0.0 & -2.9 \\
\hline & 1.508000 & -1.0 & -1.4 \\
\hline 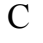 & 2.700000 & 2.329000 & -0.4 \\
\hline $\mathrm{H}$ & 1.438000 & 2.595000 & -2.291000 \\
\hline $\mathrm{H}$ & 0.670000 & 3.023000 & -0.751000 \\
\hline & 3.520000 & 1.06 & -2.509000 \\
\hline & 3.788000 & -0.487000 & -168500 \\
\hline & 2.5640 & 2.0400 & 0.545 \\
\hline
\end{tabular}




$\begin{array}{rrrr}\mathrm{H} & 3.375000 & 3.043000 & -0.989000 \\ \mathrm{C} & -1.663000 & 2.519000 & -0.554000 \\ \mathrm{C} & -1.782000 & 1.075000 & 1.372000 \\ \mathrm{C} & -1.474000 & 3.672000 & 0.467000 \\ \mathrm{H} & -2.729000 & 2.334000 & -0.754000 \\ \mathrm{H} & -1.181000 & 2.692000 & -1.518000 \\ \mathrm{C} & -1.273000 & 2.230000 & 2.282000 \\ \mathrm{H} & -2.869000 & 1.090000 & 1.230000 \\ \mathrm{H} & -1.512000 & 0.098000 & 1.786000 \\ \mathrm{H} & -2.447000 & 4.003000 & 0.860000 \\ \mathrm{H} & -0.989000 & 4.541000 & 0.012000 \\ \mathrm{H} & -0.523000 & 1.874000 & 2.994000 \\ \mathrm{H} & -2.114000 & 2.651000 & 2.852000 \\ \mathrm{C} & -1.879000 & -1.210000 & -1.844000 \\ \mathrm{C} & -2.092000 & -2.624000 & 0.152000 \\ \mathrm{C} & -3.407000 & -1.154000 & -1.698000 \\ \mathrm{H} & -1.569000 & -1.944000 & -2.601000 \\ \mathrm{H} & -1.471000 & -0.241000 & -2.148000 \\ \mathrm{C} & -3.477000 & -1.992000 & 0.452000 \\ \mathrm{H} & -2.170000 & -3.510000 & -0.490000 \\ \mathrm{H} & -1.613000 & -2.923000 & 1.085000 \\ \mathrm{H} & -3.875000 & -2.101000 & -2.008000 \\ \mathrm{H} & -3.827000 & -0.363000 & -2.325000 \\ \mathrm{H} & -3.532000 & -1.649000 & 1.490000 \\ \mathrm{H} & -4.260000 & -2.752000 & 0.305000 \\ \mathrm{C} & 1.480000 & -0.638000 & 1.704000 \\ \mathrm{C} & 0.912000 & -2.903000 & 0.948000 \\ \mathrm{C} & 2.905000 & -1.213000 & 1.637000 \\ \mathrm{H} & 1.083000 & -0.657000 & 2.729000 \\ \mathrm{H} & 1.459000 & 0.396000 & 1.345000 \\ \mathrm{C} & 2.353000 & -3.101000 & 0.416000 \\ \mathrm{H} & 0.813000 & -3.201000 & 1.999000 \\ \mathrm{H} & 0.215000 & -3.497000 & 0.358000 \\ \mathrm{H} & 3.103000 & -1.894000 & 2.480000 \\ \mathrm{H} & 3.637000 & -0.403000 & 1.669000 \\ \mathrm{H} & 2.338000 & -3.458000 & -0.619000 \\ \mathrm{H} & 2.863000 & -3.859000 & 1.031000 \\ \mathrm{~N} & 0.821000 & 0.937000 & -1.182000 \\ \mathrm{~N} & 0.592000 & -1.463000 & 0.856000 \\ \mathrm{~N} & -1.282000 & -1.610000 & -0.553000 \\ \mathrm{~N} & -1.168000 & 1.273000 & 0.051000 \\ \mathrm{O} & -3.715000 & -0.864000 & -0.356000 \\ \mathrm{O} & 3.262000 & 1.038000 & -0.455000 \\ \mathrm{O} & 3.061000 & -1.887000 & 0.411000 \\ \mathrm{O} & -0.641000 & 3.224000 & 1.511000\end{array}$

\footnotetext{
TME Carbene $G=-385461.1095 \mathrm{kcal} / \mathrm{mol}$

$\begin{array}{llll}\mathrm{C} & -0.000000 & -1.246000 & 0.000000\end{array}$

$\begin{array}{llll}\text { C } & 2.348000 & -1.364000 & -0.449000\end{array}$

$\begin{array}{llll}\text { C } & 1.384000 & 0.809000 & -0.707000\end{array}$

$\begin{array}{llll}\text { C } & 3.573000 & -0.494000 & -0.126000\end{array}$

$\mathrm{H} \quad 2.422000 \quad-1.750000 \quad-1.480000$

$\begin{array}{llll}\mathrm{H} & 2.274000 & -2.218000 & 0.228000\end{array}$

$\begin{array}{llll}\text { C } & 2.353000 & 1.461000 & 0.277000\end{array}$

$\mathrm{H} \quad 1.835000 \quad 0.761000 \quad-1.712000$
} 


$\begin{array}{rrrr}\mathrm{H} & 0.465000 & 1.383000 & -0.827000 \\ \mathrm{H} & 3.989000 & -0.032000 & -1.039000 \\ \mathrm{H} & 4.369000 & -1.099000 & 0.319000 \\ \mathrm{H} & 1.820000 & 1.891000 & 1.135000 \\ \mathrm{H} & 2.912000 & 2.273000 & -0.222000 \\ \mathrm{C} & -2.348000 & -1.364000 & 0.450000 \\ \mathrm{C} & -1.384000 & 0.809000 & 0.708000 \\ \mathrm{C} & -3.573000 & -0.494000 & 0.124000 \\ \mathrm{H} & -2.424000 & -1.748000 & 1.481000 \\ \mathrm{H} & -2.274000 & -2.218000 & -0.226000 \\ \mathrm{C} & -2.353000 & 1.461000 & -0.277000 \\ \mathrm{H} & -1.836000 & 0.761000 & 1.712000 \\ \mathrm{H} & -0.465000 & 1.382000 & 0.828000 \\ \mathrm{H} & -3.991000 & -0.032000 & 1.037000 \\ \mathrm{H} & -4.368000 & -1.099000 & -0.322000 \\ \mathrm{H} & -1.819000 & 1.892000 & -1.134000 \\ \mathrm{H} & -2.913000 & 2.273000 & 0.222000 \\ \mathrm{~N} & 1.127000 & -0.570000 & -0.287000 \\ \mathrm{~N} & -1.128000 & -0.570000 & 0.288000 \\ \mathrm{O} & 3.247000 & 0.506000 & 0.815000 \\ \mathrm{O} & -3.245000 & 0.506000 & -0.816000\end{array}$

\begin{tabular}{|c|c|c|c|}
\hline $\mathrm{C}$ & 0.427000 & 0.531000 & 0.050000 \\
\hline $\mathrm{C}$ & 0.504000 & 2.916000 & -0.506000 \\
\hline $\mathrm{C}$ & -0.306000 & 2.207000 & 1.689000 \\
\hline $\mathrm{C}$ & -0.474000 & 4.031000 & -0.846000 \\
\hline $\mathrm{H}$ & 1.382000 & 3.361000 & 0.007000 \\
\hline $\mathrm{H}$ & 0.885000 & 2.474000 & -1.436000 \\
\hline $\mathrm{C}$ & -1.677000 & 2.800000 & 2.002000 \\
\hline $\mathrm{H}$ & 0.478000 & 2.924000 & 2.008000 \\
\hline $\mathrm{H}$ & -0.172000 & 1.309000 & 2.308000 \\
\hline $\mathrm{C}$ & -1.149000 & 4.719000 & 0.339000 \\
\hline $\mathrm{H}$ & -1.256000 & 3.635000 & -1.511000 \\
\hline $\mathrm{H}$ & 0.082000 & 4.780000 & -1.430000 \\
\hline $\mathrm{C}$ & -2.205000 & 3.851000 & 1.021000 \\
\hline $\mathrm{H}$ & -1.607000 & 3.239000 & 3.009000 \\
\hline $\mathrm{H}$ & -2.415000 & 1.987000 & 2.078000 \\
\hline $\mathrm{H}$ & -0.392000 & 5.053000 & 1.068000 \\
\hline $\mathrm{H}$ & -1.633000 & 5.634000 & -0.029000 \\
\hline $\mathrm{H}$ & -2.922000 & 4.491000 & 1.554000 \\
\hline $\mathrm{H}$ & -2.787000 & 3.352000 & 0.231000 \\
\hline $\mathrm{C}$ & 2.280000 & -0.508000 & -1.222000 \\
\hline $\mathrm{C}$ & 2.735000 & 0.643000 & 0.890000 \\
\hline $\mathrm{C}$ & 3.355000 & 0.072000 & -2.133000 \\
\hline $\mathrm{H}$ & 2.661000 & -1.432000 & -0.744000 \\
\hline $\mathrm{H}$ & 1.420000 & -0.809000 & -1.834000 \\
\hline $\mathrm{C}$ & 3.726000 & 1.795000 & 0.720000 \\
\hline $\mathrm{H}$ & 3.301000 & -0.296000 & 1.045000 \\
\hline $\mathrm{H}$ & 2.159000 & 0.796000 & 1.813000 \\
\hline $\mathrm{C}$ & 4.584000 & 0.656000 & -1.437000 \\
\hline $\mathrm{H}$ & 2.905000 & 0.860000 & -2.758000 \\
\hline $\mathrm{H}$ & 3.671000 & -0.728000 & -2.820000 \\
\hline $\mathrm{C}$ & 4.299000 & 1.959000 & -0.687000 \\
\hline $\mathrm{H}$ & 4.545000 & 1.625000 & 1.436000 \\
\hline
\end{tabular}




\begin{tabular}{|c|c|c|c|}
\hline $\mathrm{H}$ & 3.254000 & 2.741000 & 1.023000 \\
\hline $\mathrm{H}$ & 5.027000 & -0.090000 & -0.756000 \\
\hline $\mathrm{H}$ & 5.347000 & 0.850000 & -2.203000 \\
\hline $\mathrm{H}$ & 5.219000 & 2.554000 & -0.615000 \\
\hline $\mathrm{H}$ & 3.604000 & 2.556000 & -1.297000 \\
\hline $\mathrm{N}$ & 1.808000 & 0.424000 & -0.215000 \\
\hline $\mathrm{N}$ & -0.063000 & 1.840000 & 0.297000 \\
\hline $\mathrm{C}$ & -0.427000 & -0.531000 & 0.050000 \\
\hline $\mathrm{C}$ & -2.280000 & 0.508000 & -1.222000 \\
\hline $\mathrm{C}$ & -2.735000 & -0.643000 & 0.890000 \\
\hline $\mathrm{C}$ & -3.356000 & -0.072000 & -2.132000 \\
\hline $\mathrm{H}$ & -2.661000 & 1.432000 & -0.744000 \\
\hline $\mathrm{H}$ & -1.420000 & 0.809000 & -1.833000 \\
\hline $\mathrm{C}$ & -3.726000 & -1.795000 & 0.720000 \\
\hline $\mathrm{H}$ & -3.301000 & 0.296000 & 1.045000 \\
\hline $\mathrm{H}$ & -2.159000 & -0.797000 & 1.813000 \\
\hline $\mathrm{C}$ & -4.584000 & -0.656000 & -1.437000 \\
\hline $\mathrm{H}$ & -2.905000 & -0.860000 & -2.758000 \\
\hline $\mathrm{H}$ & -3.671000 & 0.728000 & -2.820000 \\
\hline $\mathrm{C}$ & -4.300000 & -1.958000 & -0.687000 \\
\hline $\mathrm{H}$ & -4.545000 & -1.625000 & 1.436000 \\
\hline $\mathrm{H}$ & -3.255000 & -2.742000 & 1.023000 \\
\hline $\mathrm{H}$ & -5.027000 & 0.090000 & -0.756000 \\
\hline $\mathrm{H}$ & -5.347000 & -0.849000 & -2.203000 \\
\hline $\mathrm{H}$ & -5.220000 & -2.554000 & -0.615000 \\
\hline $\mathrm{H}$ & -3.605000 & -2.556000 & -1.297000 \\
\hline $\mathrm{N}$ & -1.808000 & -0.425000 & -0.215000 \\
\hline $\mathrm{C}$ & -0.504000 & -2.916000 & -0.506000 \\
\hline $\mathrm{C}$ & 0.307000 & -2.207000 & 1.689000 \\
\hline $\mathrm{C}$ & 0.474000 & -4.031000 & -0.846000 \\
\hline $\mathrm{H}$ & -1.382000 & -3.361000 & 0.007000 \\
\hline $\mathrm{H}$ & -0.885000 & -2.474000 & -1.435000 \\
\hline $\mathrm{C}$ & 1.678000 & -2.800000 & 2.002000 \\
\hline $\mathrm{H}$ & -0.478000 & -2.924000 & 2.008000 \\
\hline $\mathrm{H}$ & 0.172000 & -1.309000 & 2.308000 \\
\hline $\mathrm{C}$ & 1.149000 & -4.719000 & 0.339000 \\
\hline $\mathrm{H}$ & 1.256000 & -3.635000 & -1.511000 \\
\hline $\mathrm{H}$ & -0.082000 & -4.780000 & -1.430000 \\
\hline $\mathrm{C}$ & 2.205000 & -3.851000 & 1.021000 \\
\hline $\mathrm{H}$ & 1.608000 & -3.238000 & 3.009000 \\
\hline $\mathrm{H}$ & 2.415000 & -1.987000 & 2.077000 \\
\hline $\mathrm{H}$ & 0.392000 & -5.053000 & 1.068000 \\
\hline $\mathrm{H}$ & 1.633000 & -5.634000 & -0.029000 \\
\hline $\mathrm{H}$ & 2.921000 & -4.491000 & 1.555000 \\
\hline $\mathrm{H}$ & 2.788000 & -3.353000 & 0.231000 \\
\hline $\mathrm{N}$ & 0.063000 & -1.841000 & 0.297000 \\
\hline \multicolumn{4}{|c|}{$T A z E^{2+} G=-779071.6502 \mathrm{kcal} / \mathrm{mol}$} \\
\hline $\mathrm{C}$ & 0.668000 & -0.374000 & 0.465000 \\
\hline $\mathrm{C}$ & 2.954000 & -0.378000 & 1.528000 \\
\hline $\mathrm{C}$ & 1.197000 & 1.162000 & 2.329000 \\
\hline $\mathrm{C}$ & 3.985000 & 0.529000 & 0.881000 \\
\hline 11 & 3.117000 & -0.433000 & 2.611000 \\
\hline $\mathrm{H}$ & 3.060000 & -1.387000 & 1.154000 \\
\hline & 1.603 & 2.592000 & 1.9560 \\
\hline
\end{tabular}




\begin{tabular}{|c|c|c|c|}
\hline $\mathrm{H}$ & 000 & 00 & 00 \\
\hline $\mathrm{H}$ & 0.125000 & 1.123000 & 2.548000 \\
\hline & 4.057000 & 1.945000 & 1.438000 \\
\hline & 3.810000 & 0.563000 & -0.207000 \\
\hline & 4.952000 & 0.026000 & 1.017000 \\
\hline & 2.840000 & 2.790000 & 1.081000 \\
\hline & 1.751000 & 3.113000 & 2.912000 \\
\hline & 0.755000 & 3.101000 & 1.482000 \\
\hline & 4.208000 & 1.918000 & 2.528000 \\
\hline & 4.953000 & 2.424000 & \\
\hline & 3.099000 & 3.853000 & 3000 \\
\hline & 2.591000 & 2.603000 & 0.0 \\
\hline & -0.186000 & -1.594000 & -1.48800 \\
\hline & 1.713000 & -2.569000 & -0.245000 \\
\hline & 0.422000 & -1.448000 & -2.876000 \\
\hline & -0.618000 & -2.596000 & -1.359000 \\
\hline & -1.011000 & -0.881000 & -1.381000 \\
\hline & 3.007000 & -2.532000 & 000 \\
\hline & 1.10 & -3.4 & 5000 \\
\hline & 1.88 & -2.7 & 000 \\
\hline & 1.627000 & -2.321000 & -3.212000 \\
\hline & 0.707000 & -0.398000 & -3.038000 \\
\hline & -0.397000 & -1.6 & \\
\hline C & 2.883000 & -1.9 & 4000 \\
\hline $\mathrm{H}$ & 3.327000 & $-3.5^{\prime}$ & 8000 \\
\hline & & -2.0 & 000 \\
\hline & 1.39 & -3.3 & 000 \\
\hline 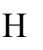 & 1.813000 & -2.2 & 6000 \\
\hline 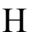 & 3.778000 & -2.15 & -3.011000 \\
\hline & 2.915000 & -0.819000 & -2.369000 \\
\hline & 0.78 & -1.4 & -0.3 \\
\hline $\mathrm{N}$ & 1.548000 & 0.089000 & 1.351000 \\
\hline $\mathrm{C}$ & -0.69 & 0.3 & 000 \\
\hline C & & & 00 \\
\hline c & -1.71 & 2.60 & 0.3 \\
\hline C & -0.55 & 2.00 & -2.555000 \\
\hline 4 & 0.500000 & 2.905000 & -0.899000 \\
\hline $\mathrm{H}$ & 0.943 & 1.22 & -1.2 \\
\hline C & -2.957000 & 2.981000 & -0.431000 \\
\hline $\mathrm{H}$ & -1.042000 & 3.462000 & 0.439000 \\
\hline $\mathrm{H}$ & -1.98 & 2.36 & 1.433000 \\
\hline C & -1.72 & $2.9^{\prime}$ & -2.682000 \\
\hline H & -0.897000 & 1.00 & -2.8 \\
\hline $\mathrm{H}$ & 0.241000 & 2.285 & -3.256000 \\
\hline C & -2.958000 & 2.548000 & -1.891000 \\
\hline $\mathrm{H}$ & -3.031000 & 4.07 & -0.387000 \\
\hline $\mathrm{H}$ & -3.870000 & 2.61 & 0.056000 \\
\hline $\mathrm{H}$ & -1.414000 & 3.990000 & -2.387000 \\
\hline $\mathrm{H}$ & -1.98 & 3.02 & -3.7 \\
\hline $\mathrm{H}$ & -3.858000 & 2.968000 & -2.353000 \\
\hline $\mathrm{H}$ & -3.076000 & 1.452000 & -1.966000 \\
\hline $\mathrm{N}$ & -0.832000 & 1.506000 & -0.103000 \\
\hline 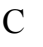 & -3.089000 & -0.023000 & 1.077000 \\
\hline $\mathrm{C}$ & -1.314000 & -1.350000 & 2.216000 \\
\hline $\mathrm{C}$ & -3.918000 & -1.174000 & 0.525000 \\
\hline & -3.434000 & 0.271000 & 2.078000 \\
\hline
\end{tabular}




$\begin{array}{rrrr}\mathrm{H} & -3.223000 & 0.828000 & 0.415000 \\ \mathrm{C} & -1.361000 & -2.816000 & 1.804000 \\ \mathrm{H} & -2.046000 & -1.165000 & 3.011000 \\ \mathrm{H} & -0.337000 & -1.120000 & 2.653000 \\ \mathrm{C} & -3.867000 & -2.514000 & 1.251000 \\ \mathrm{H} & -3.653000 & -1.326000 & -0.533000 \\ \mathrm{H} & -4.949000 & -0.796000 & 0.520000 \\ \mathrm{C} & -2.574000 & -3.278000 & 0.993000 \\ \mathrm{H} & -1.324000 & -3.365000 & 2.755000 \\ \mathrm{H} & -0.438000 & -3.090000 & 1.277000 \\ \mathrm{H} & -4.048000 & -2.384000 & 2.329000 \\ \mathrm{H} & -4.706000 & -3.117000 & 0.888000 \\ \mathrm{H} & -2.725000 & -4.345000 & 1.192000 \\ \mathrm{H} & -2.354000 & -3.213000 & -0.084000 \\ \mathrm{~N} & -1.636000 & -0.322000 & 1.182000\end{array}$

TAzE Carbene $G=-389674.9805 \mathrm{kcal} / \mathrm{mol}$

$\begin{array}{lrrr}\mathrm{C} & 0.103000 & -1.267000 & -0.624000 \\ \mathrm{C} & 1.398000 & 0.873000 & -1.283000 \\ \mathrm{C} & 2.458000 & -1.260000 & -0.708000 \\ \mathrm{C} & 1.697000 & 1.724000 & -0.054000 \\ \mathrm{H} & 2.235000 & 0.940000 & -1.994000 \\ \mathrm{H} & 0.533000 & 1.281000 & -1.805000 \\ \mathrm{C} & 2.828000 & -1.336000 & 0.769000 \\ \mathrm{H} & 3.248000 & -0.743000 & -1.270000 \\ \mathrm{H} & 2.379000 & -2.271000 & -1.121000 \\ \mathrm{C} & 2.847000 & 1.249000 & 0.833000 \\ \mathrm{H} & 0.790000 & 1.777000 & 0.569000 \\ \mathrm{H} & 1.893000 & 2.750000 & -0.400000 \\ \mathrm{C} & 2.548000 & -0.061000 & 1.564000 \\ \mathrm{H} & 3.897000 & -1.590000 & 0.832000 \\ \mathrm{H} & 2.277000 & -2.167000 & 1.228000 \\ \mathrm{H} & 3.775000 & 1.159000 & 0.244000 \\ \mathrm{H} & 3.042000 & 2.036000 & 1.574000 \\ \mathrm{H} & 3.121000 & -0.104000 & 2.500000 \\ \mathrm{H} & 1.488000 & -0.053000 & 1.865000 \\ \mathrm{C} & -2.197000 & -1.655000 & -0.200000 \\ \mathrm{C} & -1.697000 & 0.381000 & -1.466000 \\ \mathrm{C} & -2.697000 & -1.147000 & 1.141000 \\ \mathrm{H} & -3.035000 & -1.726000 & -0.914000 \\ \mathrm{H} & -1.763000 & -2.653000 & -0.084000 \\ \mathrm{C} & -1.878000 & 1.631000 & -0.598000 \\ \mathrm{H} & -2.679000 & 0.075000 & -1.851000 \\ \mathrm{H} & -1.098000 & 0.593000 & -2.358000 \\ \mathrm{C} & -3.233000 & 0.284000 & 1.144000 \\ \mathrm{H} & -1.866000 & -1.216000 & 1.862000 \\ \mathrm{H} & -3.479000 & -1.833000 & 1.497000 \\ \mathrm{C} & -2.165000 & 1.348000 & 0.875000 \\ \mathrm{H} & -2.708000 & 2.214000 & -1.025000 \\ \mathrm{H} & -0.995000 & 2.282000 & -0.663000 \\ \mathrm{H} & -4.054000 & 0.383000 & 0.414000 \\ \mathrm{H} & -3.684000 & 0.477000 & 2.126000 \\ \mathrm{H} & -2.458000 & 2.296000 & 1.347000 \\ \mathrm{H} & -1.233000 & 1.037000 & 1.376000 \\ \mathrm{~N} & -1.151000 & -0.804000 & -0.782000 \\ & & & \end{array}$




$$
\begin{array}{lrrr}
\mathrm{N} & 1.193000 & -0.557000 & -0.975000 \\
& & & \\
\text { TAzetE G }=-482535.8924 & \mathrm{kcal} / \mathrm{mol} \\
\mathrm{C} & 0.763000 & -0.076000 & 0.008000 \\
\mathrm{C} & -0.566000 & 0.209000 & -0.072000 \\
\mathrm{C} & 2.751000 & 0.878000 & 1.175000 \\
\mathrm{C} & 2.783000 & 1.072000 & -0.900000 \\
\mathrm{C} & 3.764000 & 1.495000 & 0.201000 \\
\mathrm{H} & 3.006000 & -0.169000 & 1.411000 \\
\mathrm{H} & 2.511000 & 1.415000 & 2.102000 \\
\mathrm{H} & 3.040000 & 0.085000 & -1.322000 \\
\mathrm{H} & 2.566000 & 1.776000 & -1.716000 \\
\mathrm{H} & 4.768000 & 1.063000 & 0.177000 \\
\mathrm{H} & 3.835000 & 2.583000 & 0.304000 \\
\mathrm{C} & 1.110000 & -2.452000 & 0.907000 \\
\mathrm{C} & 0.965000 & -2.318000 & -1.192000 \\
\mathrm{C} & 1.192000 & -3.491000 & -0.223000 \\
\mathrm{H} & 0.120000 & -2.428000 & 1.391000 \\
\mathrm{H} & 1.885000 & -2.481000 & 1.687000 \\
\mathrm{H} & -0.081000 & -2.244000 & -1.527000 \\
\mathrm{H} & 1.633000 & -2.235000 & -2.061000 \\
\mathrm{H} & 0.438000 & -4.282000 & -0.221000 \\
\mathrm{H} & 2.186000 & -3.937000 & -0.319000 \\
\mathrm{C} & -2.334000 & -1.164000 & 1.141000 \\
\mathrm{C} & -2.673000 & -0.917000 & -0.935000 \\
\mathrm{C} & -3.461000 & -1.606000 & 0.192000 \\
\mathrm{H} & -2.598000 & -0.284000 & 1.750000 \\
\mathrm{H} & -1.903000 & -1.925000 & 1.807000 \\
\mathrm{H} & -3.078000 & 0.065000 & -1.223000 \\
\mathrm{H} & -2.493000 & -1.496000 & -1.852000 \\
\mathrm{H} & -4.459000 & -1.215000 & 0.404000 \\
\mathrm{H} & -3.518000 & -2.692000 & 0.075000 \\
\mathrm{C} & -2.232000 & 2.068000 & 0.450000 \\
\mathrm{C} & -0.465000 & 2.774000 & -0.451000 \\
\mathrm{C} & -1.816000 & 3.431000 & -0.122000 \\
\mathrm{H} & -2.095000 & 2.011000 & 1.545000 \\
\mathrm{H} & -3.232000 & 1.687000 & 0.200000 \\
\mathrm{H} & 0.305000 & 2.965000 & 0.306000 \\
\mathrm{H} & -0.028000 & 2.941000 & -1.445000 \\
\mathrm{H} & -1.800000 & 4.275000 & 0.572000 \\
\mathrm{H} & -2.388000 & 3.699000 & -1.015000 \\
\mathrm{~N} & -1.506000 & -0.849000 & -0.040000 \\
\mathrm{~N} & -1.114000 & 1.467000 & -0.288000 \\
\mathrm{~N} & 1.718000 & 0.973000 & 0.122000 \\
\mathrm{~N} & 1.287000 & -1.390000 & -0.096000 \\
& & & \\
\mathrm{~T} A z e t E^{2+} \mathrm{G}= & -482229.0623 \mathrm{kcal} / \mathrm{mol} \\
\mathrm{C} & 0.754000 & -0.000000 & 0.001000 \\
\mathrm{C} & -0.754000 & -0.000000 & -0.000000 \\
\mathrm{H} & 2.762000 & -1.325000 & -0.964000 \\
\mathrm{H} & 0.801000 & -2.065000 & -1.505000 \\
\mathrm{H} & 3.246000 & -2.496000 & -1.820000 \\
& 3.304000 & -1.600000 & -0.054000 \\
& 0.208000 & -2.743000 & -1.503000 \\
& & & -0.910000 \\
&
\end{array}
$$




$\begin{array}{rrrr}\mathrm{H} & 0.208000 & -1.713000 & -2.356000 \\ \mathrm{H} & 2.514000 & -3.484000 & -1.441000 \\ \mathrm{H} & 2.514000 & -2.436000 & -2.875000 \\ \mathrm{C} & 2.760000 & 1.327000 & 0.964000 \\ \mathrm{C} & 0.799000 & 2.065000 & 1.507000 \\ \mathrm{C} & 2.244000 & 2.497000 & 1.821000 \\ \mathrm{H} & 3.302000 & 1.603000 & 0.054000 \\ \mathrm{H} & 3.304000 & 0.544000 & 1.503000 \\ \mathrm{H} & 0.204000 & 2.768000 & 0.912000 \\ \mathrm{H} & 0.207000 & 1.711000 & 2.358000 \\ \mathrm{H} & 2.511000 & 3.484000 & 1.443000 \\ \mathrm{H} & 2.512000 & 2.437000 & 2.876000 \\ \mathrm{C} & -2.761000 & -0.966000 & 1.323000 \\ \mathrm{C} & -0.801000 & -1.506000 & 2.064000 \\ \mathrm{C} & -2.246000 & -1.821000 & 2.495000 \\ \mathrm{H} & -3.304000 & -1.505000 & 0.540000 \\ \mathrm{H} & -3.303000 & -0.056000 & 1.597000 \\ \mathrm{H} & -0.208000 & -2.358000 & 1.713000 \\ \mathrm{H} & -0.208000 & -0.910000 & 2.767000 \\ \mathrm{H} & -2.513000 & -2.876000 & 2.435000 \\ \mathrm{H} & -2.515000 & -1.442000 & 3.482000 \\ \mathrm{C} & -2.761000 & 0.967000 & -1.324000 \\ \mathrm{C} & -0.800000 & 1.506000 & -2.065000 \\ \mathrm{C} & -2.244000 & 1.820000 & -2.497000 \\ \mathrm{H} & -3.303000 & 1.507000 & -0.543000 \\ \mathrm{H} & -3.303000 & 0.056000 & -1.597000 \\ \mathrm{H} & -0.207000 & 2.358000 & -1.714000 \\ \mathrm{H} & -0.206000 & 0.909000 & -2.767000 \\ \mathrm{H} & -2.512000 & 2.875000 & -2.439000 \\ \mathrm{H} & -2.513000 & 1.440000 & -3.484000 \\ \mathrm{~N} & -1.346000 & -0.692000 & 0.946000 \\ \mathrm{~N} & -1.345000 & 0.692000 & -0.946000 \\ \mathrm{~N} & 1.346000 & -0.948000 & -0.689000 \\ \mathrm{~N} & 1.345000 & 0.948000 & 0.691000\end{array}$

TAzetE Carbene $G=-241257.693 \mathrm{kcal} / \mathrm{mol}$

$\begin{array}{llll}\text { C } & 0.000000 & -1.093000 & -0.000000\end{array}$

$\begin{array}{llll}\text { C } & -1.552000 & 1.027000 & -0.111000\end{array}$

$\begin{array}{llll}\text { C } & -2.496000 & -0.894000 & 0.076000\end{array}$

$\begin{array}{llll}\text { C } & -3.015000 & 0.556000 & -0.031000\end{array}$

$\mathrm{H} \quad-1.250000 \quad 1.429000 \quad-1.087000$

$\mathrm{H} \quad-1.224000 \quad 1.716000 \quad 0.677000$

$\mathrm{H} \quad-2.706000 \quad-1.534000 \quad-0.791000$

$\mathrm{H} \quad-2.766000 \quad-1.438000 \quad 0.989000$

$\mathrm{H} \quad-3.621000 \quad 0.771000 \quad-0.914000$

$\begin{array}{llll}\mathrm{H} & -3.547000 & 0.908000 & 0.857000\end{array}$

$\begin{array}{llll}\text { C } & 1.552000 & 1.027000 & 0.111000\end{array}$

$\begin{array}{llll}\text { C } & 2.496000 & -0.894000 & -0.075000\end{array}$

$\begin{array}{llll}\text { C } & 3.015000 & 0.556000 & 0.030000\end{array}$

$\begin{array}{llll}\mathrm{H} & 1.250000 & 1.428000 & 1.088000\end{array}$

$\begin{array}{llll}\mathrm{H} & 1.224000 & 1.716000 & -0.676000\end{array}$

$\mathrm{H} \quad 2.706000 \quad-1.534000 \quad 0.792000$

$\mathrm{H} \quad 2.767000 \quad-1.438000 \quad-0.989000$

$\begin{array}{llll}\mathrm{H} & 3.621000 & 0.772000 & 0.913000\end{array}$

$\begin{array}{llll}\mathrm{H} & 3.546000 & 0.907000 & -0.858000\end{array}$ 


$$
\begin{array}{lrrr}
\mathrm{N} & -1.124000 & -0.386000 & 0.084000 \\
\mathrm{~N} & 1.124000 & -0.386000 & -0.084000 \\
\multicolumn{4}{c}{\mathrm{TD}} \\
\mathrm{C} & \mathrm{G}=-386657.2401 \mathrm{kcal} / \mathrm{mol} \\
\mathrm{C} & -0.775000 & 0.000000 & -0.000000 \\
\mathrm{C} & 0.589000 & 0.000000 & -0.000000 \\
\mathrm{~N} & -1.553000 & -1.112000 & 0.373000 \\
\mathrm{~N} & 1.323000 & -1.182000 & -0.192000 \\
\mathrm{~N} & -1.553000 & 1.113000 & -0.373000 \\
\mathrm{~N} & 1.323000 & 1.182000 & 0.191000 \\
\mathrm{C} & -1.008000 & -2.082000 & 1.287000 \\
\mathrm{H} & -1.834000 & -2.618000 & 1.775000 \\
\mathrm{H} & -0.353000 & -2.830000 & 0.803000 \\
\mathrm{H} & -0.418000 & -1.573000 & 2.059000 \\
\mathrm{C} & -2.471000 & -1.662000 & -0.603000 \\
\mathrm{H} & -1.988000 & -2.408000 & -1.263000 \\
\mathrm{H} & -3.310000 & -2.157000 & -0.095000 \\
\mathrm{H} & -2.869000 & -0.853000 & -1.222000 \\
\mathrm{C} & 1.213000 & -1.954000 & -1.400000 \\
\mathrm{H} & 2.158000 & -1.939000 & -1.977000 \\
\mathrm{H} & 0.972000 & -3.012000 & -1.197000 \\
\mathrm{H} & 0.418000 & -1.539000 & -2.028000 \\
\mathrm{C} & 2.451000 & -1.484000 & 0.647000 \\
\mathrm{H} & 2.402000 & -2.521000 & 1.020000 \\
\mathrm{H} & 3.414000 & -1.369000 & 0.116000 \\
\mathrm{H} & 2.459000 & -0.813000 & 1.513000 \\
\mathrm{C} & 1.215000 & 1.954000 & 1.400000 \\
\mathrm{H} & 2.160000 & 1.936000 & 1.977000 \\
\mathrm{H} & 0.975000 & 3.012000 & 1.198000 \\
\mathrm{H} & 0.419000 & 1.539000 & 2.028000 \\
\mathrm{C} & 2.451000 & 1.484000 & -0.647000 \\
\mathrm{H} & 2.404000 & 2.521000 & -1.018000 \\
\mathrm{H} & 3.414000 & 1.365000 & -0.116000 \\
\mathrm{H} & 2.457000 & 0.814000 & -1.515000 \\
\mathrm{C} & -2.470000 & 1.663000 & 0.603000 \\
\mathrm{H} & -1.988000 & 2.408000 & 1.263000 \\
\mathrm{H} & -3.309000 & 2.157000 & 0.096000 \\
\mathrm{H} & -2.868000 & 0.853000 & 1.223000 \\
\mathrm{C} & -1.008000 & 2.082000 & -1.287000 \\
\mathrm{H} & -1.834000 & 2.618000 & -1.775000 \\
\mathrm{H} & -0.353000 & 2.830000 & -0.803000 \\
\mathrm{H} & -0.418000 & 1.573000 & -2.059000 \\
& & &
\end{array}
$$

TDAE ${ }^{2+} G=-386323.3975 \mathrm{kcal} / \mathrm{mol}$

$\begin{array}{llll}\text { C } & -0.759000 & -0.000000 & 0.000000\end{array}$

$\begin{array}{llll}\text { C } & 0.759000 & -0.000000 & -0.000000\end{array}$

$\begin{array}{llll}\mathrm{N} & -1.361000 & -0.973000 & -0.670000\end{array}$

$\begin{array}{lrrr}\mathrm{N} & 1.360000 & -0.973000 & 0.670000\end{array}$

$\begin{array}{llll}\mathrm{N} & -1.360000 & 0.973000 & 0.670000\end{array}$

$\begin{array}{lrrr}\mathrm{N} & 1.360000 & 0.973000 & -0.670000\end{array}$

$\begin{array}{llll}\mathrm{C} & -2.731000 & -1.437000 & -0.394000\end{array}$

$\mathrm{H} \quad-3.438000 \quad-1.091000 \quad-1.154000$

$\mathrm{H} \quad-2.709000 \quad-2.531000 \quad-0.417000$

$\mathrm{H} \quad-3.052000 \quad-1.120000 \quad 0.599000$

C $\quad-0.660000 \quad-1.777000-1.684000$ 


$\begin{array}{rrrr}\mathrm{H} & -0.488000 & -2.792000 & -1.307000 \\ \mathrm{H} & -1.296000 & -1.841000 & -2.573000 \\ \mathrm{H} & 0.288000 & -1.323000 & -1.981000 \\ \mathrm{C} & 2.730000 & -1.437000 & 0.394000 \\ \mathrm{H} & 3.437000 & -1.091000 & 1.155000 \\ \mathrm{H} & 2.709000 & -2.531000 & 0.417000 \\ \mathrm{H} & 3.052000 & -1.120000 & -0.598000 \\ \mathrm{C} & 0.660000 & -1.778000 & 1.684000 \\ \mathrm{H} & 0.488000 & -2.792000 & 1.307000 \\ \mathrm{H} & 1.296000 & -1.840000 & 2.573000 \\ \mathrm{H} & -0.289000 & -1.324000 & 1.980000 \\ \mathrm{C} & 2.730000 & 1.437000 & -0.395000 \\ \mathrm{H} & 3.437000 & 1.092000 & -1.155000 \\ \mathrm{H} & 2.708000 & 2.531000 & -0.418000 \\ \mathrm{H} & 3.052000 & 1.120000 & 0.598000 \\ \mathrm{C} & 0.659000 & 1.777000 & -1.684000 \\ \mathrm{H} & 0.487000 & 2.792000 & -1.307000 \\ \mathrm{H} & 1.295000 & 1.841000 & -2.573000 \\ \mathrm{H} & -0.289000 & 1.323000 & -1.980000 \\ \mathrm{C} & -0.658000 & 1.778000 & 1.684000 \\ \mathrm{H} & -0.484000 & 2.791000 & 1.306000 \\ \mathrm{H} & -1.295000 & 1.843000 & 2.572000 \\ \mathrm{H} & 0.289000 & 1.323000 & 1.981000 \\ \mathrm{C} & -2.730000 & 1.437000 & 0.395000 \\ \mathrm{H} & -3.437000 & 1.092000 & 1.155000 \\ \mathrm{H} & -2.708000 & 2.531000 & 0.418000 \\ \mathrm{H} & -3.052000 & 1.120000 & -0.597000\end{array}$

TDAE Carbene $\mathrm{G}=-193308.1857 \mathrm{kcal} / \mathrm{mol}$

$\begin{array}{llll}\mathrm{C} & -0.000000 & -0.827000 & 0.000000\end{array}$

$\begin{array}{llll}\mathrm{N} & -1.161000 & -0.139000 & 0.026000\end{array}$

N $\quad \begin{array}{lll}1.161000 & -0.139000 & -0.025000\end{array}$

$\begin{array}{llll}\text { C } & -2.375000 & -0.919000 & 0.205000\end{array}$

$\mathrm{H} \quad-2.975000 \quad-0.515000 \quad 1.033000$

$\mathrm{H} \quad-2.992000 \quad-0.902000-0.706000$

$\begin{array}{llll}\mathrm{H} & -2.094000 & -1.948000 & 0.433000\end{array}$

$\begin{array}{llll}\mathrm{C} & -1.437000 & 1.250000 & -0.346000\end{array}$

$\mathrm{H} \quad-2.345000 \quad 1.270000 \quad-0.962000$

$\mathrm{H} \quad-1.612000 \quad 1.901000 \quad 0.522000$

$\mathrm{H} \quad-0.625000 \quad 1.662000 \quad-0.948000$

$\begin{array}{llll}\mathrm{C} & 1.437000 & 1.250000 & 0.346000\end{array}$

$\mathrm{H} \quad 2.345000 \quad 1.270000 \quad 0.962000$

$\mathrm{H} \quad 1.612000 \quad 1.901000 \quad-0.522000$

$\begin{array}{llll}\mathrm{H} & 0.626000 & 1.662000 & 0.949000\end{array}$

C $\quad 2.375000 \quad-0.919000 \quad-0.205000$

$\mathrm{H} \quad 2.974000 \quad-0.515000 \quad-1.033000$

$\mathrm{H} \quad 2.993000 \quad-0.902000 \quad 0.706000$

$\mathrm{H} \quad 2.093000 \quad-1.948000 \quad-0.433000$

TDAE2.0 G $=-385168.1532 \mathrm{kcal} / \mathrm{mol}$

$\begin{array}{llll}\mathrm{C} & -0.672000 & -0.027000 & -0.045000\end{array}$

$\begin{array}{llll}\text { C } & -2.872000 & 0.652000 & -0.310000\end{array}$

$\begin{array}{llll}\text { C } & -2.656000 & -0.751000 & -0.864000\end{array}$

$\begin{array}{llll}\mathrm{H} & -3.414000 & 0.617000 & 0.655000\end{array}$

$\mathrm{H} \quad-3.433000 \quad 1.308000 \quad-0.987000$ 


$\begin{array}{rrrr}\mathrm{H} & -3.491000 & -1.432000 & -0.670000 \\ \mathrm{H} & -2.485000 & -0.711000 & -1.949000 \\ \mathrm{C} & 0.672000 & -0.027000 & 0.045000 \\ \mathrm{C} & 2.872000 & 0.652000 & 0.310000 \\ \mathrm{C} & 2.656000 & -0.751000 & 0.864000 \\ \mathrm{H} & 3.414000 & 0.617000 & -0.655000 \\ \mathrm{H} & 3.434000 & 1.309000 & 0.987000 \\ \mathrm{H} & 3.491000 & -1.432000 & 0.670000 \\ \mathrm{H} & 2.486000 & -0.711000 & 1.949000 \\ \mathrm{~N} & 1.505000 & 1.128000 & 0.112000 \\ \mathrm{~N} & 1.436000 & -1.220000 & 0.205000 \\ \mathrm{~N} & -1.436000 & -1.220000 & -0.205000 \\ \mathrm{~N} & -1.505000 & 1.128000 & -0.112000 \\ \mathrm{C} & -1.371000 & 2.078000 & 0.980000 \\ \mathrm{H} & -1.962000 & 2.975000 & 0.760000 \\ \mathrm{H} & -1.725000 & 1.649000 & 1.937000 \\ \mathrm{H} & -0.324000 & 2.367000 & 1.110000 \\ \mathrm{C} & 1.371000 & 2.078000 & -0.980000 \\ \mathrm{H} & 1.962000 & 2.975000 & -0.761000 \\ \mathrm{H} & 1.725000 & 1.649000 & -1.937000 \\ \mathrm{H} & 0.323000 & 2.367000 & -1.110000 \\ \mathrm{C} & 1.674000 & -1.918000 & -1.057000 \\ \mathrm{H} & 2.221000 & -1.306000 & -1.799000 \\ \mathrm{H} & 2.248000 & -2.832000 & -0.864000 \\ \mathrm{H} & 0.707000 & -2.200000 & -1.484000 \\ \mathrm{C} & -1.674000 & -1.918000 & 1.057000 \\ \mathrm{H} & -2.221000 & -1.306000 & 1.799000 \\ \mathrm{H} & -2.248000 & -2.832000 & 0.864000 \\ \mathrm{H} & -0.707000 & -2.200000 & 1.484000\end{array}$

TDAE2. $0^{2+} \mathrm{G}=-384846.4223 \mathrm{kcal} / \mathrm{mol}$

$\begin{array}{llll}\mathrm{C} & -0.744000 & -0.000000 & -0.000000\end{array}$

$\begin{array}{llll}\text { C } & -2.898000 & 0.602000 & -0.477000\end{array}$

$\begin{array}{llll}\text { C } & -2.898000 & -0.602000 & 0.477000\end{array}$

$\begin{array}{llll}\mathrm{H} & -3.305000 & 1.514000 & -0.024000\end{array}$

$\mathrm{H} \quad-3.431000 \quad 0.416000 \quad-1.415000$

$\mathrm{H} \quad-3.431000 \quad-0.416000 \quad 1.414000$

$\mathrm{H} \quad-3.305000 \quad-1.514000 \quad 0.024000$

$\begin{array}{llll}\text { C } & 0.744000 & -0.000000 & -0.000000\end{array}$

$\begin{array}{llll}\text { C } & 2.898000 & -0.602000 & -0.477000\end{array}$

$\begin{array}{llll}\mathrm{C} & 2.898000 & 0.602000 & 0.477000\end{array}$

$\mathrm{H} \quad 3.305000 \quad-1.514000 \quad-0.024000$

$\mathrm{H} \quad 3.431000 \quad-0.416000 \quad-1.414000$

$\begin{array}{llll}\mathrm{H} & 3.431000 & 0.416000 & 1.414000\end{array}$

$\mathrm{H} \quad 3.305000 \quad 1.514000 \quad 0.024000$

$\mathrm{N} \quad \begin{array}{llll}1.458000 & -0.809000 & -0.757000\end{array}$

$\begin{array}{llll}\mathrm{N} & 1.458000 & 0.809000 & 0.757000\end{array}$

$\begin{array}{llll}\mathrm{N} & -1.458000 & -0.809000 & 0.757000\end{array}$

$\begin{array}{llll}\mathrm{N} & -1.458000 & 0.809000 & -0.757000\end{array}$

$\begin{array}{llll}\mathrm{C} & -0.995000 & 1.878000 & -1.633000\end{array}$

$\mathrm{H} \quad-1.478000 \quad 1.778000 \quad-2.610000$

$\mathrm{H} \quad-1.256000 \quad 2.852000-1.205000$

$\mathrm{H} \quad 0.088000 \quad 1.817000 \quad-1.771000$

C $\quad 0.995000 \quad-1.878000 \quad-1.633000$

$\mathrm{H} \quad 1.476000 \quad-1.776000 \quad-2.610000$ 


$\begin{array}{rrrl}\mathrm{H} & 1.259000 & -2.852000 & -1.207000 \\ \mathrm{H} & -0.088000 & -1.818000 & -1.769000 \\ \mathrm{C} & 0.995000 & 1.878000 & 1.634000 \\ \mathrm{H} & 1.477000 & 1.777000 & 2.610000 \\ \mathrm{H} & 1.257000 & 2.852000 & 1.206000 \\ \mathrm{H} & -0.088000 & 1.817000 & 1.770000 \\ \mathrm{C} & -0.995000 & -1.878000 & 1.634000 \\ \mathrm{H} & -1.477000 & -1.777000 & 2.610000 \\ \mathrm{H} & -1.258000 & -2.852000 & 1.206000 \\ \mathrm{H} & 0.088000 & -1.817000 & 1.770000\end{array}$

TDAE2.0 Carbene $G=-192577.0291 \mathrm{kcal} / \mathrm{mol}$

$\begin{array}{llll}\mathrm{C} & 0.000000 & 1.026000 & 0.000000\end{array}$

$\begin{array}{lrrr}\text { C } & -0.759000 & -1.221000 & 0.065000\end{array}$

$\begin{array}{llll}\text { C } & 0.759000 & -1.221000 & -0.065000\end{array}$

$\mathrm{H} \quad-1.101000 \quad-1.591000 \quad 1.045000$

$\mathrm{H} \quad-1.265000 \quad-1.811000-0.710000$

$\mathrm{H} \quad 1.265000 \quad-1.811000 \quad 0.710000$

$\mathrm{H} \quad 1.101000 \quad-1.590000-1.046000$

$\begin{array}{llll}\mathrm{N} & 1.069000 & 0.208000 & 0.071000\end{array}$

$\begin{array}{llll}\mathrm{N} & -1.069000 & 0.208000 & -0.071000\end{array}$

$\begin{array}{llll}\mathrm{C} & -2.437000 & 0.648000 & -0.005000\end{array}$

$\mathrm{H} \quad-3.030000 \quad 0.213000 \quad-0.821000$

$\mathrm{H} \quad-2.905000 \quad 0.359000 \quad 0.949000$

$\begin{array}{llll}\mathrm{H} & -2.453000 & 1.737000 & -0.090000\end{array}$

$\begin{array}{llll}\text { C } & 2.437000 & 0.648000 & 0.004000\end{array}$

$\mathrm{H} \quad 3.030000 \quad 0.213000 \quad 0.821000$

$\mathrm{H} \quad 2.904000 \quad 0.359000 \quad-0.950000$

$\begin{array}{llll}\mathrm{H} & 2.453000 & 1.737000 & 0.090000\end{array}$ 


\section{SXXIII. References}

1. Harris, R. K.; Becker, E. D.; Cabral De Menezes, S. M.; Granger, P.; Hoffman, R. E.; Zilm, K. W. Further Conventions for NMR Shielding and Chemical Shifts (IUPAC Recommendations 2008). Pure Appl. Chem. 2008, 80, 59-84.

2. Anka-Lufford, L. L.; Huihui, K. M. M.; Gower, N. J.; Ackerman, L. K. G.; Weix, D. J. Nickel-Catalyzed CrossElectrophile Coupling with Organic Reductants in Non-Amide Solvents. Chem. Eur. J. 2016, 22, 11564-11567.

3. Suzuki, N.; Hofstra, J. L.; Poremba, K. E.; Reisman, S. E. Nickel-Catalyzed Enantioselective Cross-Coupling of NHydroxyphthalimide Esters with Vinyl Bromides. Org. Lett. 2017, 19, 2150-2153.

4. Ortgies, S.; Depken, C.; Breder, A. Oxidative Allylic Esterification of Alkenes by Cooperative Selenium-Catalysis Using Air as the Sole Oxidant. Org. Lett. 2016, 18, 2856-2859.

5. Alder, R. W.; Blake, M. E.; Bufali, S.; Butts, C. P.; Orpen, A. G.; Schütz, J.; Williams, S. J. Preparation of Tetraalkylformamidinium Salts and Related Species as Precursors to Stable Carbenes. J. Chem. Soc., Perkin Trans. 1 2001, 1586-1593.

6. Davies, I. W.; Wu, J.; Marcoux, J.-F.; Taylor, M.; Hughes, D.; Reider, P. J.; Deeth, R. J. Experimental and Theoretical Studies on the Oxidative Addition of Palladium(0) to $\beta$-Chlorovinamidinium Salts. Tetrahedron 2001, 57, 5061-5066.

7. The same reaction was scaled up using $77 \mathrm{~g}$ of piperidin-1-ylmethylidenepiperidinium hexafluorophosphate and afforded $17 \mathrm{~g} \mathrm{(40 \% )} \mathrm{of} \mathrm{product.} \mathrm{The} \mathrm{slightly} \mathrm{lower} \mathrm{yield} \mathrm{on} \mathrm{the} \mathrm{larger} \mathrm{scale} \mathrm{was} \mathrm{likely} \mathrm{caused} \mathrm{by} \mathrm{a} \mathrm{problem} \mathrm{with} \mathrm{the}$ ${ }^{\mathrm{n}} \mathrm{BuLi}$ used to prepare the LDA.

8. Charboneau, D. J.; Barth, E. L.; Hazari, N.; Uehling, M. R.; Zultanski, S. L. A Widely Applicable Dual Catalytic System for Cross-Electrophile Coupling Enabled by Mechanistic Studies. ACS Catal. 2020, 10, 12642-12656.

9. (a) García-Domínguez, A.; Li, Z.; Nevado, C. Nickel-Catalyzed Reductive Dicarbofunctionalization of Alkenes. $J$. Am. Chem. Soc. 2017, 139, 6835-6838; (b) Shu, W.; García-Domínguez, A.; Quirós, M. T.; Mondal, R.; Cárdenas, D. J.; Nevado, C. Ni-Catalyzed Reductive Dicarbofunctionalization of Nonactivated Alkenes: Scope and Mechanistic Insights. J. Am. Chem. Soc. 2019, 141, 13812-13821; (c) Wei, X.; Shu, W.; García-Domínguez, A.; Merino, E.; Nevado, C. Asymmetric Ni-Catalyzed Radical Relayed Reductive Coupling. J. Am. Chem. Soc. 2020, 142, 1351513522.

10. Liao, J.; Basch, C. H.; Hoerrner, M. E.; Talley, M. R.; Boscoe, B. P.; Tucker, J. W.; Garnsey, M. R.; Watson, M. P. Deaminative Reductive Cross-Electrophile Couplings of Alkylpyridinium Salts and Aryl Bromides. Org. Lett. 2019, 21, 2941-2946.

11. Hoerrner, M. E.; Baker, K. M.; Basch, C. H.; Bampo, E. M.; Watson, M. P. Deaminative Arylation of Amino Acid-Derived Pyridinium Salts. Org. Lett. 2019, 21, 7356-7360.

12. Ji, L.; Qiao, J.; Li, A.; Jiang, Z.; Lu, K.; Zhao, X. Metal-Free Synthesis of Unsymmetrical Selenides from Pyridinium Salts and Diselenides Catalysed by Visible Light. Tetrahedron Lett. 2021, 72, 153071.

13. Pulikottil, F. T.; Pilli, R.; Suku, R. V.; Rasappan, R. Nickel-Catalyzed Cross-Coupling of Alkyl Carboxylic Acid Derivatives with Pyridinium Salts via C-N Bond Cleavage. Org. Lett. 2020, 22, 2902-2907.

14. Ociepa, M.; Turkowska, J.; Gryko, D. Redox-Activated Amines in $\mathrm{C}\left(\mathrm{sp}^{3}\right)-\mathrm{C}(\mathrm{sp})$ and $\mathrm{C}\left(\mathrm{sp}^{3}\right)-\mathrm{C}\left(\mathrm{sp}^{2}\right)$ Bond Formation Enabled by Metal-Free Photoredox Catalysis. ACS Catal. 2018, 8, 11362-11367.

15. Konishi, A.; Hirao, Y.; Nakano, M.; Shimizu, A.; Botek, E.; Champagne, B.; Shiomi, D.; Sato, K.; Takui, T.; Matsumoto, K.; Kurata, H.; Kubo, T. Synthesis and Characterization of Teranthene: A Singlet Biradical Polycyclic Aromatic Hydrocarbon Having Kekulé Structures. J. Am. Chem. Soc. 2010, 132, 11021-11023.

16. Guo, P.; Wang, K.; Jin, W.-J.; Xie, H.; Qi, L.; Liu, X.-Y.; Shu, X.-Z. Dynamic Kinetic Cross-Electrophile Arylation of Benzyl Alcohols by Nickel Catalysis. J. Am. Chem. Soc. 2021, 143, 513-523.

17. Sha, S.-C.; Tcyrulnikov, S.; Li, M.; Hu, B.; Fu, Y.; Kozlowski, M. C.; Walsh, P. J. Cation- $\pi$ Interactions in the Benzylic Arylation of Toluenes with Bimetallic Catalysts. J. Am. Chem. Soc. 2018, 140, 12415-12423.

18. Bering, L.; Jeyakumar, K.; Antonchick, A. P. Metal-Free C-O Bond Functionalization: Catalytic Intramolecular and Intermolecular Benzylation of Arenes. Org. Lett. 2018, 20, 3911-3914.

19. Willcox, D. R.; Nichol, G. S.; Thomas, S. P. Borane-Catalyzed C( $\left.\mathrm{sp}^{3}\right)-$ F Bond Arylation and Esterification Enabled by Transborylation. ACS Catal. 2021, 11, 3190-3197.

20. Shi, J.; Yuan, T.; Zheng, M.; Wang, X. Metal-Free Heterogeneous Semiconductor for Visible-Light Photocatalytic Decarboxylation of Carboxylic Acids. ACS Catal. 2021, 11, 3040-3047.

21. CrysAlis Pro, Rigaku OD, The Woodlands, Texas, 2015.

22. Sheldrick, G. M. A Short History of SHELX. Acta Cryst. 2008, A64, 112-122.

23. Spek, A. L. PLATON SQUEEZE: A Tool for the Calculation of the Disordered Solvent Contribution to the Calculated Structure Factors. Acta Cryst. C 2015, 71, 9-18. 
24. Frisch, M. J.; Trucks, G. W.; Schlegel, H. B.; Scuseria, G. E.; Robb, M. A.; Cheeseman, J. R.; Scalmani, G.; Barone, V.; Petersson, G. A.; Nakatsuji, H.; Li, X.; Caricato, M.; Marenich, A. V.; Bloino, J.; Janesko, B. G.; Gomperts, R.; Mennucci, B.; Hratchian, H. P.; Ortiz, J. V.; Izmaylov, A. F.; Sonnenberg, J. L.; Williams; Ding, F.; Lipparini, F.; Egidi, F.; Goings, J.; Peng, B.; Petrone, A.; Henderson, T.; Ranasinghe, D.; Zakrzewski, V. G.; Gao, J.; Rega, N.; Zheng, G.; Liang, W.; Hada, M.; Ehara, M.; Toyota, K.; Fukuda, R.; Hasegawa, J.; Ishida, M.; Nakajima, T.; Honda, Y.; Kitao, O.; Nakai, H.; Vreven, T.; Throssell, K.; Montgomery Jr., J. A.; Peralta, J. E.; Ogliaro, F.; Bearpark, M. J.; Heyd, J. J.; Brothers, E. N.; Kudin, K. N.; Staroverov, V. N.; Keith, T. A.; Kobayashi, R.; Normand, J.; Raghavachari, K.; Rendell, A. P.; Burant, J. C.; Iyengar, S. S.; Tomasi, J.; Cossi, M.; Millam, J. M.; Klene, M.; Adamo, C.; Cammi, R.; Ochterski, J. W.; Martin, R. L.; Morokuma, K.; Farkas, O.; Foresman, J. B.; Fox, D. J. Wallingford, CT, 2016.

25. Zhao, Y.; Truhlar, D. G. The M06 Suite of Density Functionals for Main Group Thermochemistry, Thermochemical Kinetics, Noncovalent Interactions, Excited States, and Transition Elements: Two New Functionals and Systematic Testing of Four M06-Class Functionals and 12 Other Functionals. Theor. Chem. Acc. 2008, 120, 215241.

26. Francl, M. M.; Pietro, W. J.; Hehre, W. J.; Binkley, J. S.; Gordon, M. S.; DeFrees, D. J.; Pople, J. A. Self-Consistent Molecular Orbital Methods. XXIII. A Polarization-Type Basis Set for Second-Row Elements. J. Chem. Phys. 1982, 77, 3654-3665.

27. Wanzlick, H. W.; Schikora, E. Ein Neuer Zugang zur Carben-Chemie. Angew. Chem. 1960, 72, 494-494.

28. Huynh, M. T.; Anson, C. W.; Cavell, A. C.; Stahl, S. S.; Hammes-Schiffer, S. Quinone $1 \mathrm{e}^{-}$and $2 \mathrm{e}^{-} / 2 \mathrm{H}^{+}$Reduction Potentials: Identification and Analysis of Deviations from Systematic Scaling Relationships. J. Am. Chem. Soc. 2016, $138,15903-15910$. 